\title{
A MICROFABRICATED PLATFORM FOR THREE-DIMENSIONAL MICROSYSTEMS
}

\author{
By
}

\author{
GRANT A. McCALLUM
}

\section{SUBMITTED IN PARTIAL FULFILLMENT OF THE REQUIREMENTS FOR THE DEGREE OF DOCTOR OF PHILOSOPHY}




\section{CASE WESTERN RESERVE UNIVERSITY \\ SCHOOL OF GRADUATE STUDIES}

We hereby approve the thesis/dissertation of

Grant A. McCallum

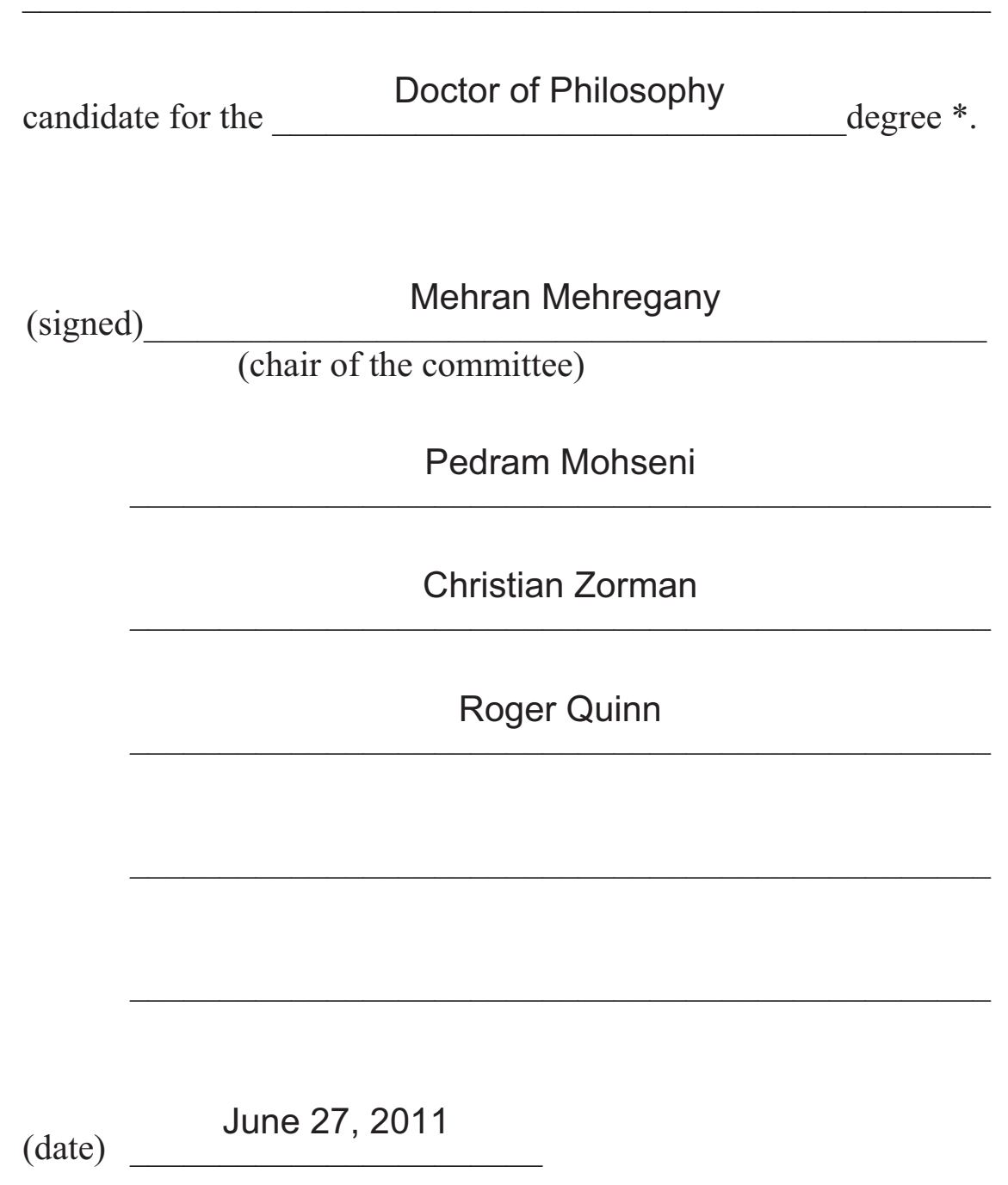

*We also certify that written approval has been obtained for any proprietary material contained therein. 
Copyright (९ 2011 by Grant McCallum \& Mehran Mehregany

All rights reserved 
To Christina and my family. 


\section{Table of Contents}

\section{List of Tables...........................................................................................}

List of Figures .............................................................................................. vii

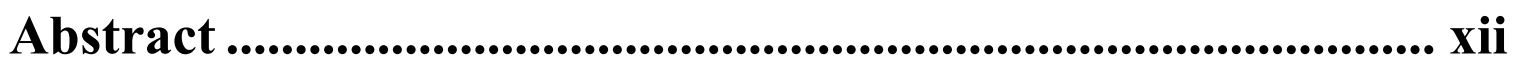

Chapter 1: Introduction ....................................................................1

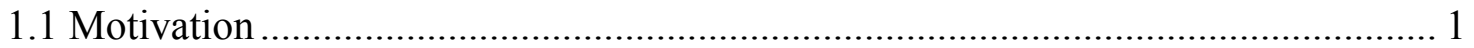

1.2 Review of Existing Sensor Platforms …………….............................................. 1

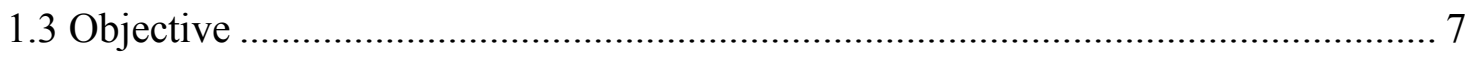

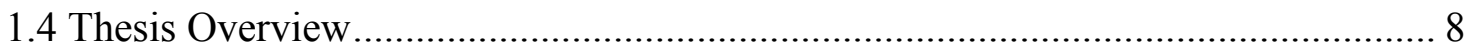

Chapter 2: Platform Design and Structural Modeling ..............10

2.1 Design of Hook-Hole Connection Features .......................................................... 11

2.1.1 Hook-Hole Concept..................................................................................... 11

2.1.2 Finite-Element Modeling of Hook-Hole Features........................................... 15

2.2 Connection Design Using Interdigitated Teeth (i.e., No Hook-Hole

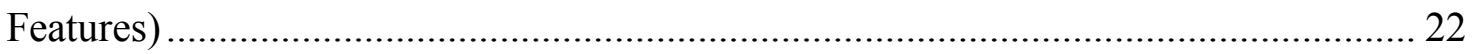

2.2.1 Specifications for Three Different Configurations ......................................... 22

2.2.2 Description of Finite-Element Modeling Simulation Conditions...................... 23

2.2.3 Finite-Element Modeling Results .............................................................. 25

2.3 Platform Structural Mechanics FEM Analysis..................................................... 34

2.3.1 Pressure and Point Force Simulation Results ................................................. 35

2.3.2 Edge Force Finite Element Simulation Results ................................................. 40

2.3.3 Analytical Verification of Finite Element Simulation Results ......................... 42

2.4 Platform Size Reduction and Mechanical Strength Analysis................................... 44

2.4.1 Finite-Element Modeling Simulations............................................................ 44

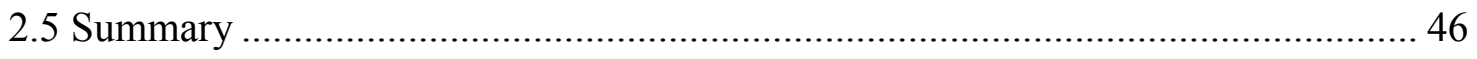

Chapter 3: Platform Microfabrication ..........................................50

3.1 Hook-Hole Microfabrication Processs ................................................................ 50

3.2 Interdigitated Teeth Microfabrication Process (i.e. No Hook-Hole Features) ........ 53

Chapter 4: Platform Assembly Procedures ....................................55 
4.1 Investigating Different Solder and Assembly Jig Material Choices ..................... 55

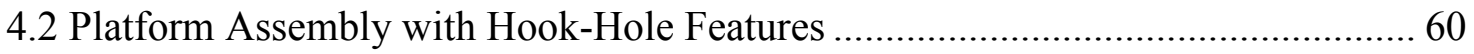

4.2.1 Female and Male Component Assembly with Hook-Hole Features ................ 60

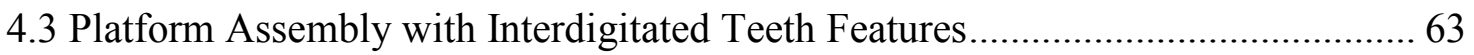

4.3.1 Integrated-Circuit and Passive Component Soldering Process ....................... 63

4.3.2 Process for Assembling Platform Segment Pieces Together ............................ 65

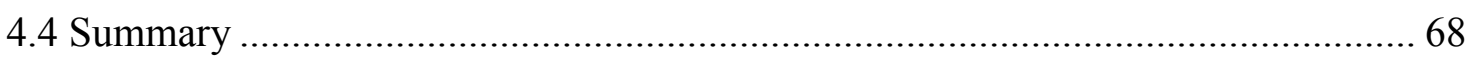

\section{Chapter 5: Inertial Measurement Unit (IMU) System Development for Using Platform ..................................................70}

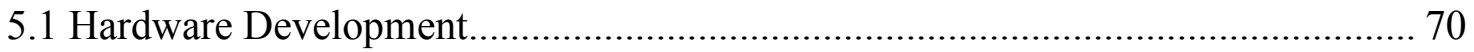

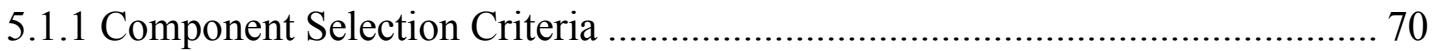

5.1.2 Microcontroller to Sensor Interface Development Board ............................. 74

5.1.3 Hardware for Transferring Data to External Personal Computer (PC) ........... 76

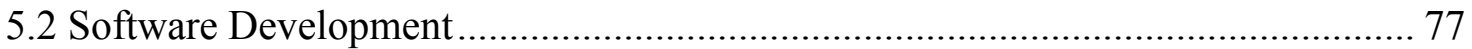

5.2.1 Software for Data-logging Functionality................................................. 78

5.2.2 Software to Retrieve Platform Data and Store on a PC ................................ 84

5.3 Integrated Meso-Scale Sensing Platform ....................................................... 87

5.3.1 Meso-scale Platform: System Schematic and Board Design......................... 88

5.4 Microsystem Platform Implementation of the IMU ........................................... 91

\section{Chapter 6: Microsystem Testing and Electrical Properties} Assessment ...............................................................................93

6.1 IMU Platform Static Acceleration Testing....................................................... 93

6.2 Platform Electrical Properties Assessment ...................................................... 96

6.2.1 Resistive/Capacitive Delays on the I2C Clock and Data Bus Lines ............... 96

6.2.2 Power Consumption and Maximum Operating Times ................................. 100

6.3 Platform Antenna Integration for Wireless Communication ............................. 102

Chapter 7: Conclusion and Future Work...................................105

7.1 Future Work ............................................................................................ 106

Appendix A: Microfabrication Details...........................................110

CASE WESTERN RESERVE UNIVERSITY FABRICATION DETAILS ............ 110

A.1.1 Positive Photoresist AZ9260 (DRIE Processing) ....................................... 110 
A.1.2 Positive Photoresist AZ9245 (5 $\mu \mathrm{m}$ thick photoresist) Metal Lift-Off .......... 110

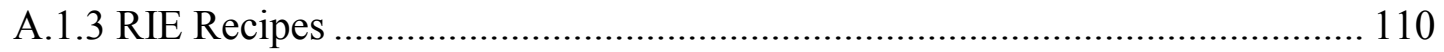

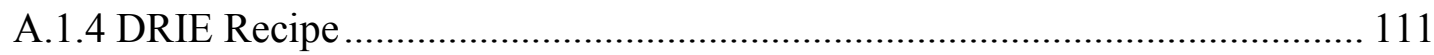

A.1.5 PECVD Oxide TEOS Recipe (1800 ̊̊/min deposition rate) ......................... 112

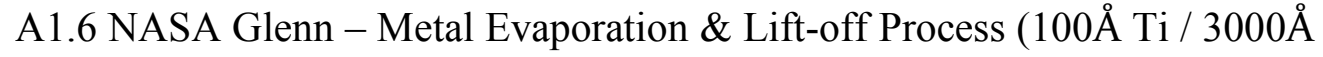

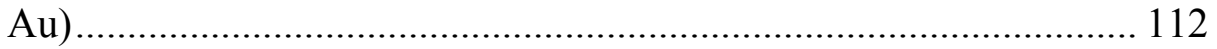

Appendix B: C-Program for Platform Data-Logging ..............113

Appendix C: C-Program for RS-232 Data Transmission......132

Appendix D: Perl Program for Sensor Data Conversion .......141

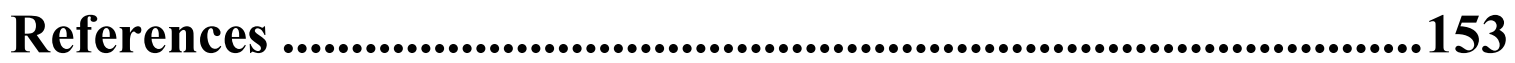




\section{List of Tables}

Table 2.1: Top compression and shear pressure simulation results for the three teeth designs ...................................................................... 26

Table 2.2: Side compression and shear pressure simulation results for the

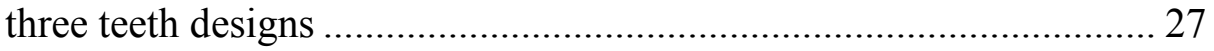

Table 2.3: End cap compression and shear pressure simulation results for the

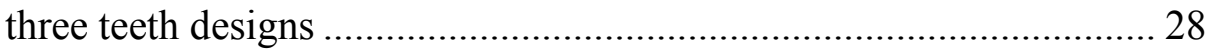

Table 2.4: Top compression and shear point force simulation results for the three teeth designs ...................................................................... 29

Table 2.5: Side compression and shear point force simulation results for the three teeth designs ...................................................................... 30

Table 2.6: Side compression and shear point force simulation results for the three teeth designs ................................................................ 31

Table 2.7: Top-Side compression and shear edge force simulation results for the three teeth designs ..................................................................... 32

Table 2.8: Top-Front compression and shear edge force simulation results for the three teeth designs ................................................................... 33

Table 2.9: Side-Front compression and shear edge force simulation results for the three teeth designs ................................................................... 34

Table 2.10: Pressure, compression stress simulation results ................................. 36

Table 2.11: Pressure, shear stress simulation results........................................... 37

Table 2.12: Point force, compression stress simulation results............................. 38

Table 2.13: Point force, shear stress simulation results........................................ 39

Table 2.14: Edge force, compression stress simulation results. ............................ 40

Table 2.15: Edge force, shear stress simulation results ...................................... 41

Table 2.16: Comparison between simulation and analytical calculated stress

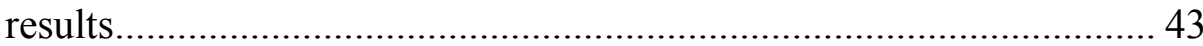

Table 2.17: Simulation results for area force applied to a $1.5 \mathrm{~mm}^{3}$ cube, 5 $\mathrm{mm}^{3}$ cube, $12 \mathrm{~mm}^{3}$ cube and $12 \times 12 \times 25 \mathrm{~mm}^{3}$ platform structure........ 45

Table 4.1: Solder compositions investigated for platform electrical connectivity 
Table 4.2: Summary of materials and equipment used to assemble both platform connection types. ........................................................... 69

Table 6.1: Calculation of I2C RC delays for different pull-up resistor configurations.

Table 6.2: Maximum platform data-logging duration for 2, 100 and $145 \mathrm{~Hz}$ sampling rates and 2, 4, 8, 16, and 32 Mbit Flash memory sizes...... 101 


\section{List of Figures}

Figure 1.1: Exemplifying protective packaging in sensing microsystems: (a) environmental sensing system [28]; and (b) implantable sensing

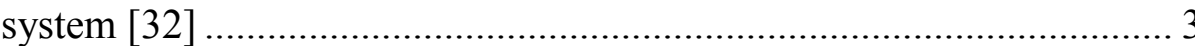

Figure 1.2: Flexible PCB-based sensing system in the form of a cube [34] .............. 4

Figure 1.3: 3-D ultrasonic transducer array for multidirectional ultrasonic imaging [35] ......................................................................... 5

Figure 1.4: 3-D microfabricated package with integrated sensors and

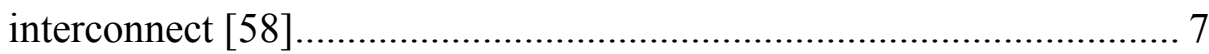

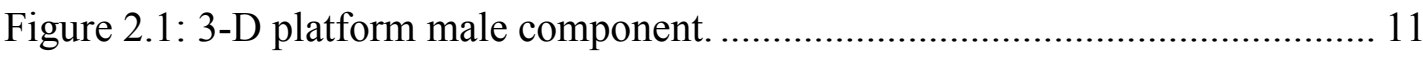

Figure 2.2: 3-D platform female component ...................................................... 12

Figure 2.3: Close-up of "Hook" features (all units are in millimeters) .................... 13

Figure 2.4: Close-up image of "Hole" features (all units are in millimeters) ........... 13

Figure 2.5: 3-D platform end cap component.................................................... 14

Figure 2.6: Complete 3-D platform components prior to assembly ........................ 14

Figure 2.7: Hook designs: (a) non-flush; and (b) flush. ....................................... 16

Figure 2.8: Side views of maximum stress and displacement with applied force to the Hook-end for the: (a) flush design; and (b) nonflush design

Figure 2.9: Simulation results for maximum stress (top) and maximum displacement (bottom) versus applied force for both flush and non-flush Hook structures.

Figure 2.10: Dynamic 3-D stress-strain simulation results for flush Hooks and Holes with widths (a) $160 \mu \mathrm{m}$, (b) $170 \mu \mathrm{m}$ and (c) $180 \mu \mathrm{m} \ldots \ldots \ldots . .20$

Figure 2.11: Results for dynamic, 3-D stress-strain simulation as a function of Hole width: (top) maximum stress; and (bottom) maximum displacement.

Figure 2.12: Three interdigitated teeth designs: (a) $1 \mathrm{~mm}$ teeth length; (b) 2 $\mathrm{mm}$ teeth length; and (c) $0.5 \mathrm{~mm}$ teeth length

Figure 2.13: Pressure and point force simulation configurations where the blue surface is fixed and the green surface has the applied force:
(a) Top Compression;
(b) Side Compression;
(c) Endcap 
Compression; (d) Top Shear; (e) Side Shear; and (f) End cap Shear

Figure 2.14: Edge force simulation configurations where the blue surface is fixed: (a) Top-Side Edge Compression; (b) Top-Front Edge Compression; (c) Side-Front Edge Compression; (d) Top-Side Edge Shear; (e) Top-Front Edge Shear; (f) Side-Front Edge Shear.

Figure 2.15: Top side compression and shear pressure simulation results for the three teeth designs 25

Figure 2.16: Side compression and shear pressure simulation results for the three teeth designs

Figure 2.17: End cap compression and shear pressure simulation results for the three teeth designs

Figure 2.18: Top compression and shear point force simulation results for the three teeth designs 28

Figure 2.19: Side compression and shear point force simulation results for the three teeth designs 29

Figure 2.20: End cap compression and shear point force simulation results for the three teeth designs

Figure 2.21: Top-Side compression and shear edge force simulation results for the three teeth designs.

Figure 2.22: Top-Front compression and shear edge force simulation results for the three teeth designs. 32

Figure 2.23: Side-Front compression and shear edge force simulation results for the three teeth designs. 33

Figure 2.24: Pressure, compression stress simulation results.................................... 36

Figure 2.25: Pressure, shear stress simulation results ............................................... 37

Figure 2.26: Point force, compression stress simulation results................................. 38

Figure 2.27: Point force, shear stress simulation results. .......................................... 39

Figure 2.28: Edge force, compression stress simulation results ................................ 40

Figure 2.29: Edge force, shear stress simulation results............................................ 41

Figure 2.30: Different platform realizations: (a) $12 \times 12 \times 25 \mathrm{~mm}^{3}$; (b) $12 \mathrm{~mm}^{3}$; (c) $5 \mathrm{~mm}^{3}$ cube; and (d) $1.5 \mathrm{~mm}^{3}$ cube. All have $1 \mathrm{~mm}$ interdigitated teeth features except for the $1.5 \mathrm{~mm}^{3}$ cube which has $0.5 \mathrm{~mm}$ features 44 
Figure 2.31: Platform size comparisons for simulated stress versus applied pressure.

Figure 3.1: Fabrication steps for the Hook-Hole platform : (a) Thermal silicon dioxide; (b) Ti/Au metal deposition; (c) PECVD silicon dioxide; (d) silicon dioxide window RIE; (e) silicon dioxide field RIE; (f) front-side DRIE and (g) backside DRIE 52

Figure 3.2: Interdigitated teeth platform fabrication process: (a) thermal

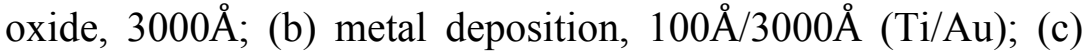
PECVD TEOS oxide deposition (5400A) and BOE etch; (d) metal deposition, $100 \AA / 3000 \AA$ (Ti/Au); (e) PECVD TEOS oxide. (5400A) and BOE etch; (f) Field Oxide BOE etch; and (g) Frontside DRIE etch $(250 \mu \mathrm{m})$

Figure 3.3: Interdigitated teeth platform - 4-inch wafer before DRIE processing

Figure 4.1: Different solder types investigated: (a) prefabricated spheres; (b) paste; and (c) ribbon 56

Figure 4.2: Orthogonal solder joints connecting male and end cap components

Figure 4.3: Jig material tested for platform assembly: (a) FR-4; and (b) aluminum. (Macor ${ }^{\mathrm{TM}}$ material is not shown in this figure)

Figure 4.4: Hook test structure (left) being inserted into Hole test structures (right) to examine component assembly

Figure 4.5: Fully-assembled cube structure using only Hook-Hole connectors 62

Figure 4.6: Hook-Hole mechanical assembly steps to create the platform structure

Figure: 4.7: Procedure to solder passive and IC components to the platform segment pieces: (a) segment placed on silicon handle wafer; (b) solder paste applied to passive bond pad locations; (c) passive components placed on platform segment; (d) solder paste placed on IC bond pad locations; (e) IC components hand placed on platform segment; (f) tweezers used to place wafer on hotplate; (g) wait until solder reflows $(20 \mathrm{sec})$; (h) tweezers used to pick up completed platform segment after removing the silicon wafer from the hotplate and allowed to cool for a minute

Figure 4.8: Completed platform segments with all passive and IC components soldered 
Figure 4.9: Process to assemble interdigitating teeth platform: (a) join two segments together on the FR-4 jig; (b) place drops of Silastic ${ }^{\mathrm{TM}}$ at the seam created by the two segments; (c) place jig under IRheater to cure Silastic ${ }^{\mathrm{TM}}$; (d) repeat process to attach an end cap segment to the platform; (e) assembly is halfway complete; (f) place preformed solder spheres with TACFLUX ${ }^{\mathrm{TM}}$ on orthogonal solder pad locations; (g) place platform on silicon wafer then on hotplate; (h) wait until the solder spheres reflow and orthogonal solder joints are created (upper left-hand corner)

Figure 4.10: The platform with integrated battery holder exposed and the fully-assembled platform............................................................ 68

Figure 5.1: SoftBaugh, Inc. ES2274 development board ................................... 72

Figure 5.2: Custom sensor development board ................................................... 75

Figure 5.3: I2C connections to sensor development board .................................. 75

Figure 5.4: RS-232 development board connections........................................... 76

Figure 5.5: Complete software development system.............................................. 78

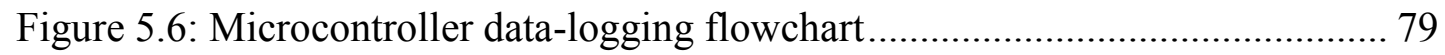

Figure 5.7: Sensor data retrieval via an RS-232 connection .................................. 85

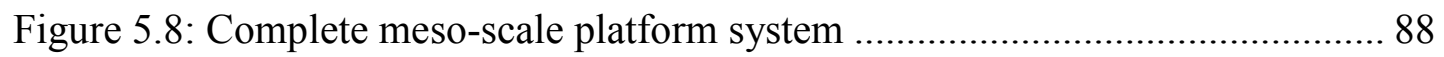

Figure 5.9: Schematic of complete meso-scale sensing platform ........................... 89

Figure 5.10: Spy-Bi-Wire connections for microcontroller programming............... 90

Figure 5.11: Connections for data retrieval via RS-232 interface ........................... 90

Figure 5.12: Three-dimensional view of the proposed silicon-based platform ......... 91

Figure 5.13: Two-dimensional representation of the IMU platform system ............. 92

Figure 6.1: Two-degree-of-freedom test fixture for static IMU tests ........................ 93

Figure 6.2: Platform secured on two-degree-of-freedom test jig for static

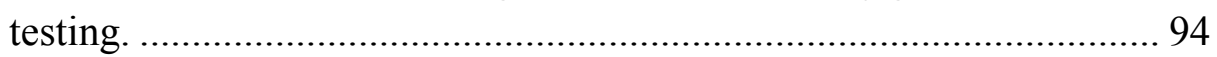

Figure 6.3: Test rotation protocol for the static IMU testing................................. 95

Figure 6.4: Captured platform acceleration data from repeated motion test jig experiments. Numbered periods refer to the rotation test position from Fig. 6.3 .................................................................. 96

Figure 6.5: PCB platform with additional capacitors to mimic the silicon

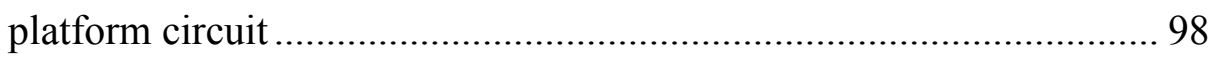


Figure 6.6: Successfully captured PCB platform data with an additional capacitance of $570 \mathrm{pF}$ and $940 \mathrm{pF}$ on I2C clock and data lines, respectively

Figure 6.7: Current consumption for different platform components during data-logging operation at $100 \mathrm{~Hz}$ data-acquisition rate

Figure 6.8: Three-dimensional antenna design for a non-uniform meander line [77]

Figure 6.9: $135 \mathrm{GHz}$ co-planar patch antenna with polymer-filled cavity [82] ..... 104

Figure 7.1: Concept drawing of a hybrid substrate material platform configuration: (a) 2-D unassembled platform segments; (b) 3-D assembled platform showing the $\mathrm{SiC}$ sensor segment and two glass segments; (c) 3-D assembled platform showing the $\mathrm{SiC}$ sensor segment, SOI antenna segment and glass segment with solar cells for power generation 
A Microfabricated Platform for Three-Dimensional Microsystems

Abstract

by

\section{GRANT A. McCALLUM}

This dissertation presents a platform structure fabricated from silicon wafers that, once assembled, forms a three-dimensional (3-D) structure that fully houses a microsystem containing sensors and the necessary system electronics including the power supply. The unique design of this platform provides a supporting package in a 3-D form factor, as well as routing capabilities with orthogonal mechanical and electrical connections between the assembled internal sides.

Two different mechanical connector types are investigated to aide in assembly: (i) Hook-Hole snap lock features; and (ii) interdigitated teeth. Both connection types are analyzed to characterize structural strength and identify optimal platform sizes. The results indicate that, under simulated pressures and forces, the platform mechanical strength is enhanced nearly three to five times depending on the connector type. Furthermore, the mechanical strength is orders of magnitude greater for smaller platform sizes. 
An example platform is fabricated to realize an autonomous data-logging inertial measurement unit. The unit consists of a programmable microcontroller, Flash memory, a three-axis accelerometer and a three-axis gyroscope. Double-sided polished, $250 \mu \mathrm{m}$ thick, (100) and (111) silicon wafers are processed using standard microfabrication techniques to produce the individual platform pieces. The platform pieces are manually assembled and incorporate out-of-plane, orthogonal solder joints to enable stable mechanical and electrical connectivity between the individual platform pieces.

Sensor data, at a rate of $100 \mathrm{~Hz}$, is collected via a microcontroller and stored in the on-board flash memory. The platform is mounted on a two-degree-of-freedom rotational stage and manually rotated in $90^{\circ}$ increments such that the sensor's $\mathrm{x}, \mathrm{y}$ and z-axes experience $+\lg$ and/or $-1 \mathrm{~g}$ accelerations. The platform structure can collect reliable and repeatable inertial data. The system electrical properties are characterized including the maximum power consumption of $\sim 16 \mathrm{~mW}$ and the duration of data-logging capability which is $\sim 48$ hours.

It is envisioned that extensions of this 3-D platform, combined with standard microfabrication techniques, will enable the integration of a variety of heterogeneous materials and devices with a form factor that reduces planar footprint and expands 3-D design space. Through-wafer vias can be used to electrically connect devices fabricated into both wafer surfaces or assembled on these surfaces. 


\section{Chapter 1}

\section{Introduction}

\subsection{Motivation}

Sensing microsystems, whether they are connected via a wireless network or operate autonomously, are created and deployed to gain a better understanding of the physical phenomena occurring around them. Since sensing microsystems also inhabit a three-dimensional (3-D) environment, why have they been mostly confined to planer surfaces with unidirectional sensing functionality? Can additional applications not be realized if a microsystem platform was deployed that enabled true, $360^{\circ}$ sensing? Furthermore, can a platform structure be directly assembled using the micromachined sensor substrate, therefore, eliminating the need for any additional packaging? It seems possible to create a system package while simultaneously micromachining the sensors, electronics and interconnects required to create a functional 3-D microsystem.

\subsection{Review of Existing Sensor Platforms}

Since the 1980's, sensing platforms have been used in many different applications. Early sensing platforms were used for monitoring oceanic phenomenon [15]. Since then they have found many uses in different applications including environmental monitoring [6-12], consumer applications [13] and automotive [14]. Most recently, there is an increased interest for using sensing platforms in the areas of structural health monitoring [15-20] and biomedical/physiological monitoring [21-26]. 
Sensing platforms need to be located in close proximity to the phenomena of interest and be of relatively small size as to not be a disturbance in the environment it monitors. To address these requirements, two classes of sensing strategies have emerged: (1) wireless sensor networks; and (2) autonomous, data-logging sensing systems. Wireless sensor networks are associated with sensing over a large geographical area in which thousands of sensor platforms, more specifically called sensor nodes, are scattered about an area in fixed locations [27], e.g., in medical applications where the network resides within a hospital for patient monitoring [28]. The sensed data is wirelessly transmitted from the sensor nodes to a central location for storage and processing. In either scenario, the sensors are placed in strategic locations for proper data-acquisition. Generally, these types of sensor platforms are larger because of the battery sizes needed to satisfy the wireless communication requirements over long periods of time [29].

The second sensor platform class is autonomous, data-logging systems. These platforms are generally smaller in size because the sensed data is stored locally to a memory device and not wirelessly transmitted which requires additional functionality and power leading to larger battery sizes. Also, data-logging systems are generally used for short, continuous sensing sessions where the post-processed data is used to identify an anomaly in a mechanical structure [30] or human physiology [31]. Generally, these sensing platforms move through the environment of interest instead of being fixed in one location or installed and then removed for interrogation.

In either class of sensing platforms, there are two physical characteristics they share in common. The first similarity is the additional packaging required to complete the sensing platform. Figure 1.1(a) shows the additional protective packaging 
encapsulating the sensors used in an environmental sensing application [28]. Figure 1.1(b) shows a packaging scheme for a biomedical, implantable sensing device [32].

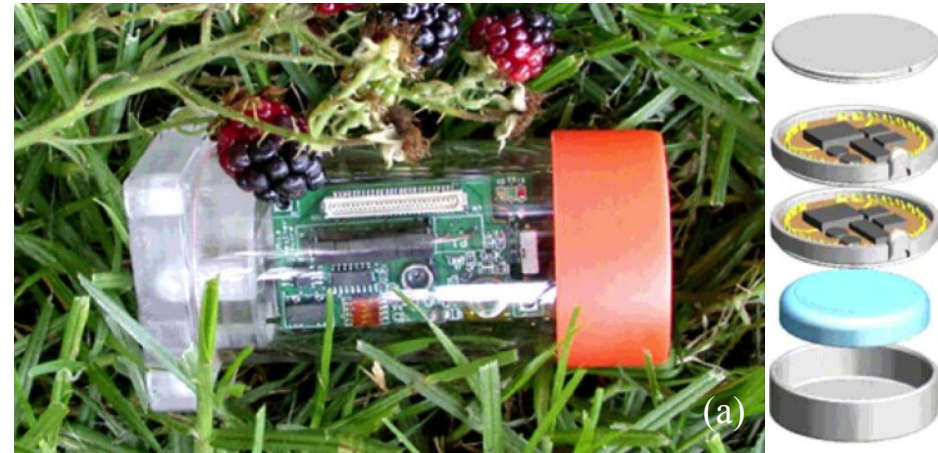

Figure 1.1: Exemplifying protective packaging in

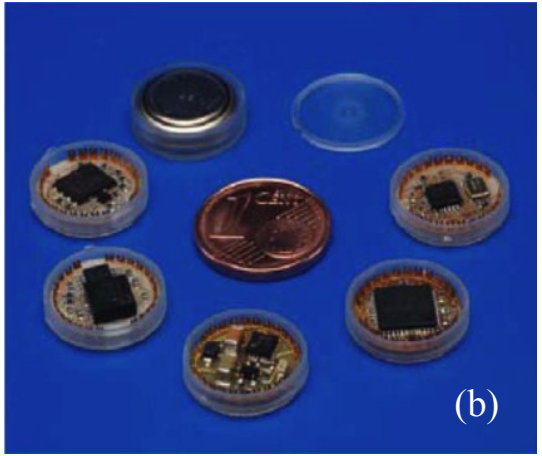

in sensing microsystems: environmental sensing system [28]; and (b) implantable sensing system [32].

One can see that the packaged sensors and electrical components are placed on printed-circuit-boards (PCBs) and then encapsulated with another layer of protective packaging which greatly increases the size and cost of the sensing system. The system in Figure 1.1(b) employs the concept of three-dimensional (3-D) stacking where different functional pieces of the system are interconnected together in a vertical manner to create the compact two-dimensional footprint. This concept has been applied to die-stacking in which individual integrated circuit (IC) dies are stacked on top of each other using an intermediate interposer layer to properly route the different signals between the stacked dies [33].

Another sensing platform shown in Fig. 1.2 was created using a flexible PCB that recorded and wirelessly transmitted temperature, humidity and luminance data [34]. The flexible PCB was folded into the shape of a $20 \mathrm{~mm}$ cube with the coin-cell batteries placed within the platforms cube structure. Although the batteries are protected within the platform, additional packaging is required to protect the ICs from the environment. In 
addition, all the sensors are located on one side of the sensing platform limiting its ability to perform multi-directional sensing which is the second physical characteristic inherent to most sensor platforms.

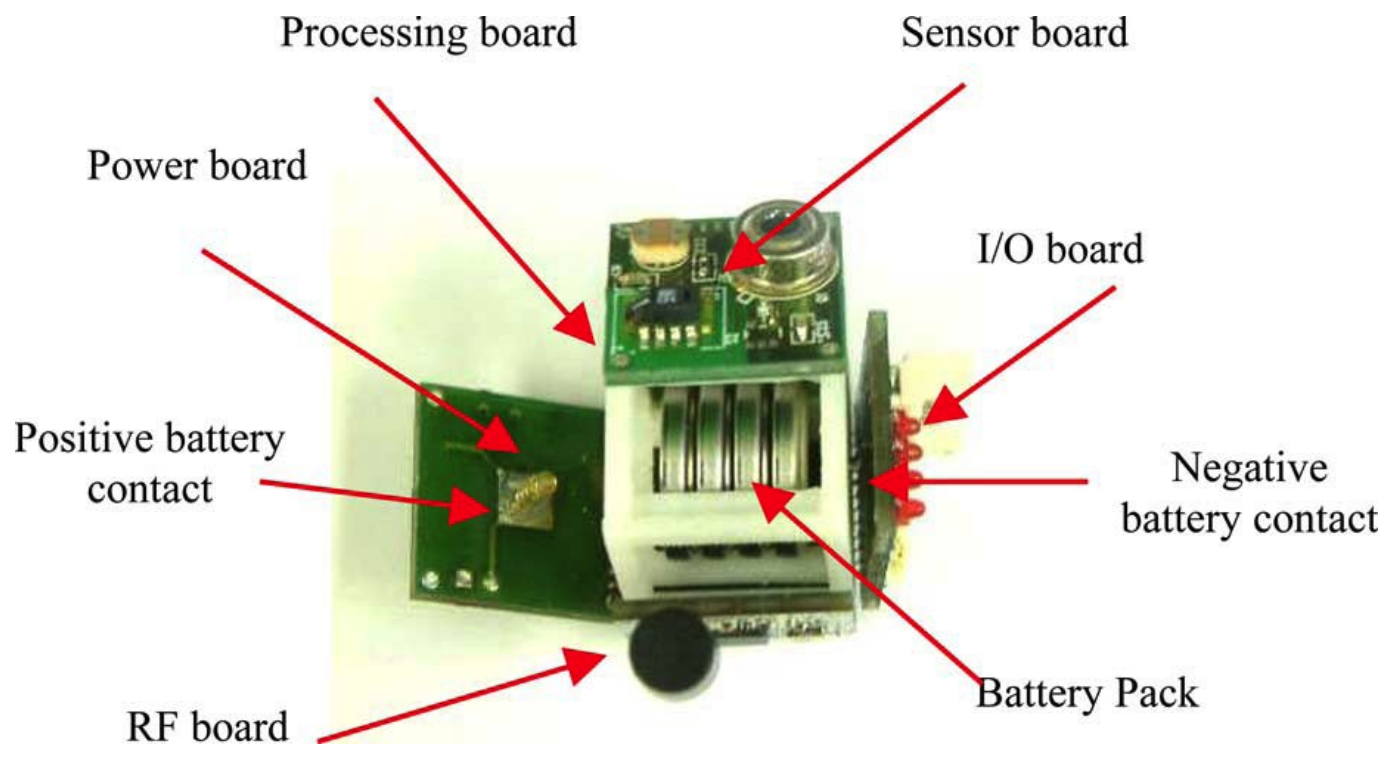

Figure 1.2: Flexible PCB-based sensing system in the form of a cube [34].

Applications exist where platform structures that have true 3-D sensing and actuation capabilities would be advantageous. For example, a 3-D ultrasonic imaging platform has been demonstrated by using planar, micromachined, capacitive array transducers wrapped around a hexagonal silicone structure to simultaneously image in seven directions as shown in Fig. 1.3 [35]. Each of the (total) 1240 transducer elements are independently controlled via a dedicated bond pad with no integrated support electronics. This platform's multi-directional imaging functionality proves to be more practical and beneficial than typical unidirectional, micromachined ultrasonic imaging platforms. This observation is especially true for medical applications such as intravascular ultrasound and capsule ultrasound endoscopy. 


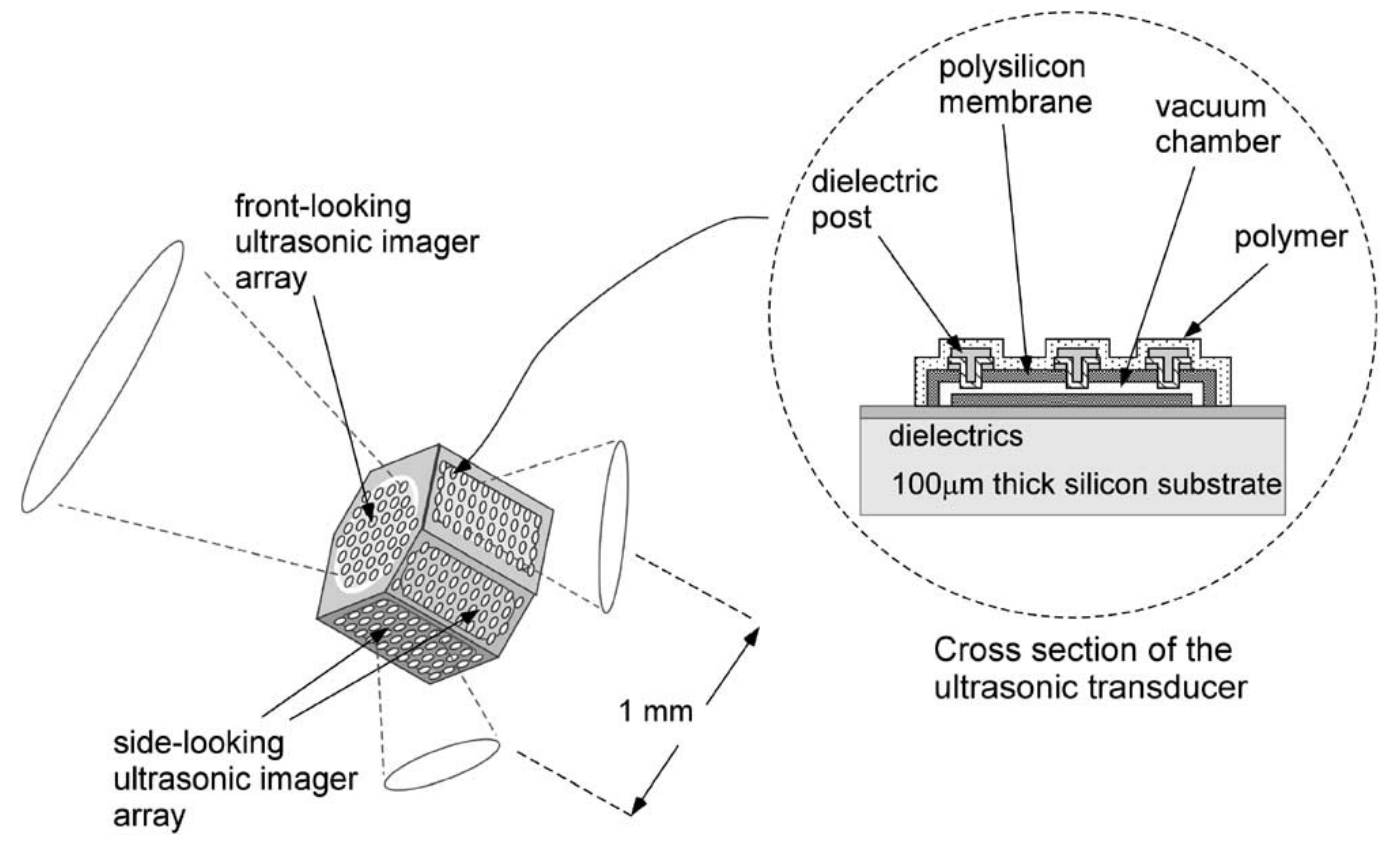

Figure 1.3: 3-D ultrasonic transducer array for multidirectional ultrasonic imaging [35].

Another application is for next generation deep brain stimulation (DBS) electrodes. Within the past 15 years, DBS has been used clinically to treat many types of neurological conditions. The most prevalent condition is motor function disorders such as Parkinson's Disease [36-39] and other emerging treatments for pain [40-42], epilepsy [43-45] and psychiatric disorders [46-49].

DBS produces a localized electrical field which can either inhibit or elicit neuronal activity in targeted brain structures to produce a desired outcome. The cylindrical shape of existing electrode sites produces a uniform electrical stimulation field circumferentially around the electrode [50-52]. This uniform electrical field produces uniform current distributions and can lead to adverse post-operative effects if the current spreads to unintended neuronal structures due to improper electrode 
placement. Some known adverse effects are: tonic muscle contraction, dysarthria, paraesthesia, ocular deviation, ipsilateral mydriasis and worsening of akinesia [53].

To prevent these adverse effects, the ability to have the electrode provide current steering is highly desirable so specific neuronal structures can be targeted for stimulation while adjacent neuronal structures are unaffected [54-56]. A reported DBS electrode platform attempts to provide this current steering ability by having 16-independantly controlled electrode sites that can create specific electrical field patterns for targeted stimulation [57].

Another 3-D microsystem platform that most closest resembles the research herein is depicted in Fig. 1.4; it is a proposed micromachined WIMS cube made out of polycrystalline diamond that contains interconnects, sensors and energy-scavenging devices integrated in a package [58]. However, at the time of this writing, no specific application is known to be demonstrated with this 3-D WIMS system. Although energyharvesting has gained much attention as being a renewable and green energy source, microelectromechanical system (MEMS) based harvesting devices have yet to produce energy densities required to power real world applications [59, 60]. Nevertheless, advances are being made to create ultra-low power circuits and radio-frequency energy scavenging strategies that may, eventually, enable microsystems to operate at these extremely low energy densities [61, 62].

Some of the perceived weaknesses of the WIMS Cube are the stability and robustness of the interconnections between the cube structure and the functional dies "dropped" into the cube. This is achieved by press-fitting flexible Parylene cables 
fabricated on the sidewall pieces to the functional die pads. These flexible connectors, in addition to flexible intra-Cube sidewall connectors, were reported to be fragile and prone to tearing during assembly and require a custom multi-tip vacuum pick for handling.

In one embodiment, the WIMS Cube is fabricated out of polycrystalline diamond, which prohibits eventual fabrication of integrated circuits of MEMS sensors/actuators into the sidewalls and/or base pieces of the Cube [58]. This severally limits the future integration density of the platform, limited to the number of dies that can fit within the Cube's volume. In another embodiment, the Cube is fabricated using silicon wafers similar to the research herein [63]. However, the sidewalls are dedicated for intra-die communication and additional functionality cannot be easily integrated into the Cube structure. Furthermore, the Cube can only be constructed from a single wafer source and cannot facilitate a heterogeneous structure made from different substrate materials.
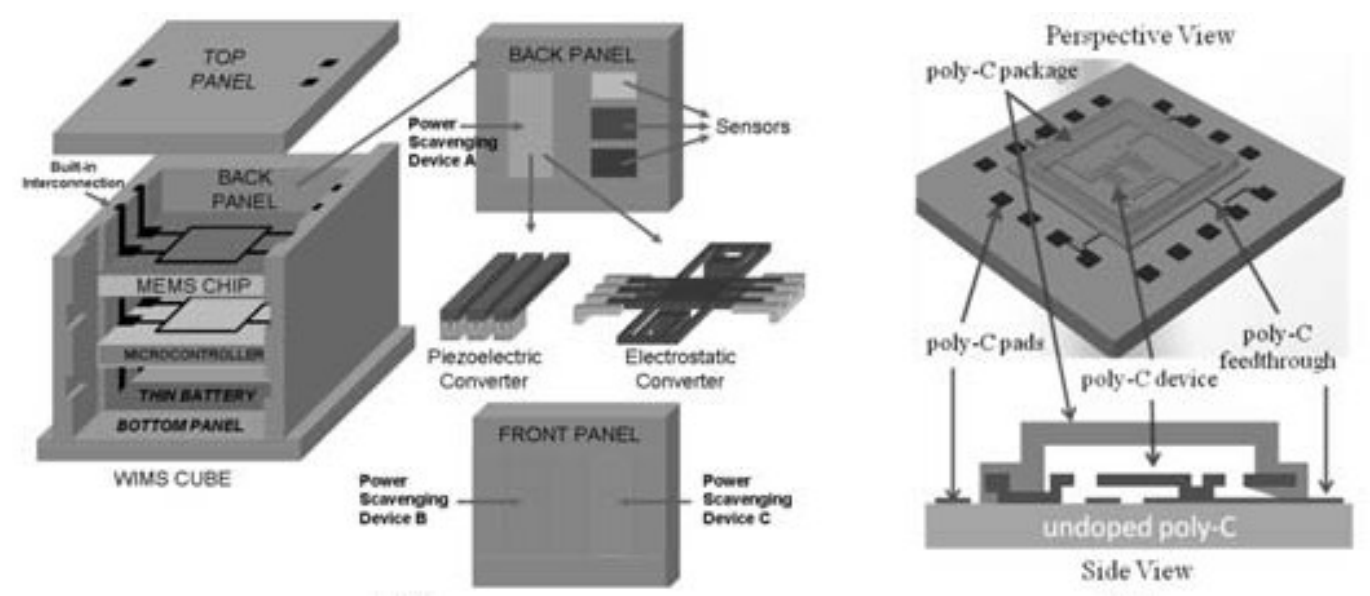

Figure 1.4: 3-D microfabricated package with integrated sensors and interconnect [58].

\subsection{Objective}

The study presented herein describes the development of a microfabricated 3-D platform for microsystem applications. The platform structure is fabricated from silicon 
wafers and assembled into a completely enclosed rectangular box or cube. Two types of platform connection strategies are investigated that will enable a simple assembly process, and robust and stable mechanical and electrical intra-platform connections. In addition, both platform types provide the ability to create heterogeneous platform structures, if desired. By targeting these design specifications, this platform structure eliminates the limitations of other 3-D platforms noted above and expands the design space for applications requiring 3-D functionality.

This 3-D platform concept is demonstrated by implementing a fully-functional, autonomous, inertial measurement unit (IMU) that records three-axis acceleration and three-axis rotation rate data. All the electronic components, sensors, electrical interconnect and power supply will be contained inside the platform structure.

\subsection{Thesis Overview}

In Chapter 2, the platform concept is described for two different types of connection strategies: (i) Hook-Hole and (ii) interdigitated teeth. In addition, finiteelement modeling is performed to verify the mechanical integrity of the platform assembly strategies.

In Chapter 3, the microfabrication processes for both platform connection types are described. The two microfabrication processes are very similar but differences occur based on the connection strategy implemented and the electrical routing density, which requires different numbers of metal layers. 
Chapter 4 discusses the different solder and jig materials investigated in order to successfully assemble the platform into a 3-D structure. Soldering techniques and methods are described which permit the intra-platform electrical communication between platform components. Finally, the assembly process for both platform connection types are discussed along with a summary of the final materials and equipment used for assembly.

Chapter 5 details the successful implementation of meso-scale IMU system using printed-circuit boards as the platform substrate. The hardware and microcontroller software development is described, which creates a system that records and stores threeaxis acceleration and three-axis rotation rate data to a Flash memory device. Once the system data-logging is complete, the microcontroller is reconfigured to transmit the stored, on-board data via a RS-232 connection to an external personal computer. Finally, implementing the meso-scale IMU system on the 3-D silicon platform is discussed.

In Chapter 6, the 3-D silicon platform IMU system is demonstrated using a standard bench top experiment for proof-of-concept. Also, an assessment of the electrical properties will be presented to define the operating parameters of the platform.

In Chapter 7, a summary of the contributions of this thesis is presented. In addition, possible future advancement opportunities are outlined. 


\section{Chapter 2}

\section{Platform Design and Structural Modeling}

A 3-D platform with MEMS sensors fabricated on the external faces and system electronics and electrical routing within the platform's three-dimensional volume, can enable information gathering from the surrounding environment in a unique fashion. Such a platform is composed of a supporting package, routing and interconnects, various sensors, processing hardware and software related to the specific application. In this chapter, the overall configuration and creation of a 3-D platform is introduced and accompanied by finite element analysis simulation results to verify design limitations and viability.

First, the Hook-Hole connector concept of the supporting package is presented which creates the male, female and end cap components using a silicon wafer as the platform material. This is followed by a discussion on a second connector concept which uses interdigitated teeth instead of the Hook-Hole design. Three different teeth configurations are discussed and mechanical simulations with full, three-dimensional platform structures are performed. The next section compares finite-element simulation results between platform structures with the Hook-Hole design and the interdigitated teeth implementation. Finally, finite-element simulations are used to determine the mechanical strength differences when the platform size is reduced from a $12 \times 12 \times 25$ $\mathrm{mm}^{3}$ box to $12 \mathrm{~mm}^{3}, 5 \mathrm{~mm}^{3}$ and $1.5 \mathrm{~mm}^{3}$ cubes. 


\subsection{Design of Hook-Hole Connection Features}

\subsubsection{Hook-Hole Concept}

Silicon is widely known for its ability to become mechanically flexible when micro-machined in certain physical dimensions [64-66]. Taking advantage of this property, the idea to micromachine Hook-and-Hole structures around the periphery of the individual platform components was developed to aid in holding the components together during the platform soldering process. The Hook features were created on platform pieces called "male components" and the Hole features were created on platform pieces called "female components" as shown in Figs. 2.1 and 2.2, respectively.

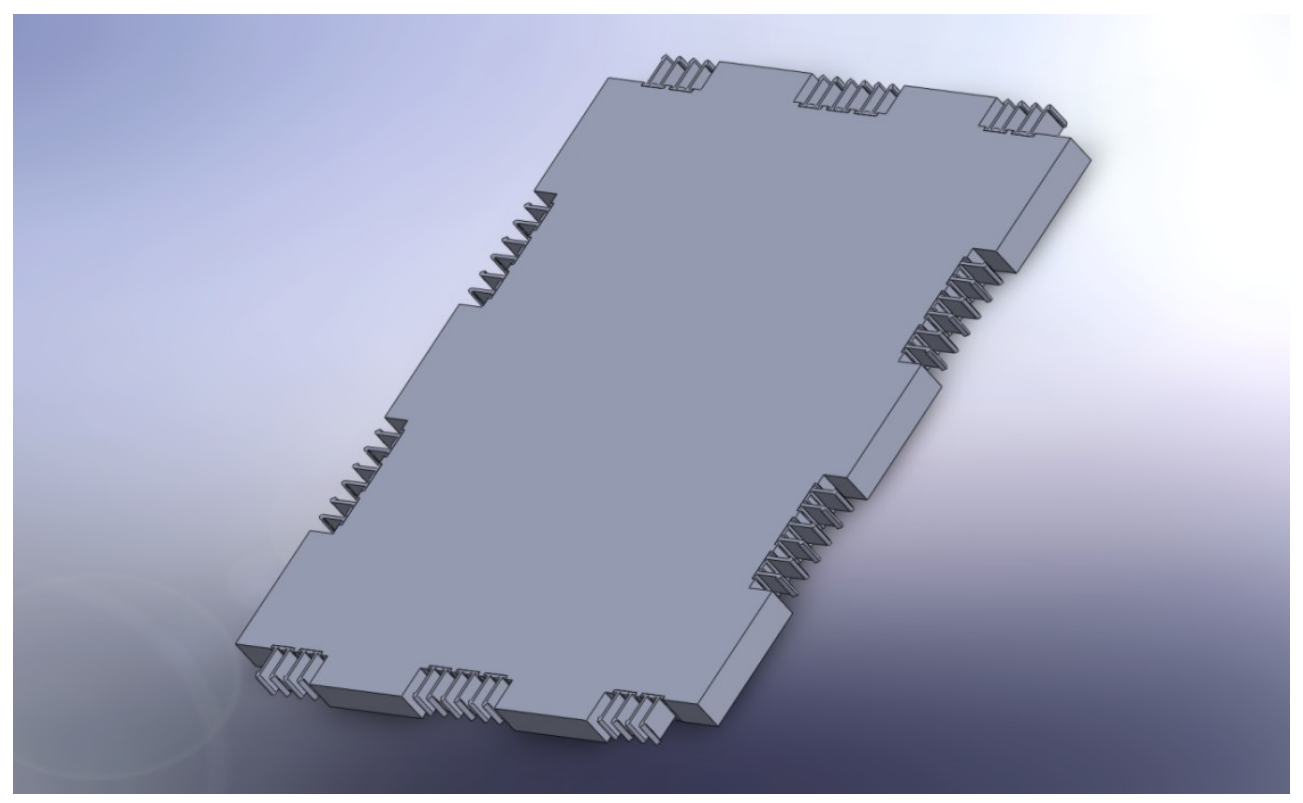

Figure 2.1: 3-D platform male component. 


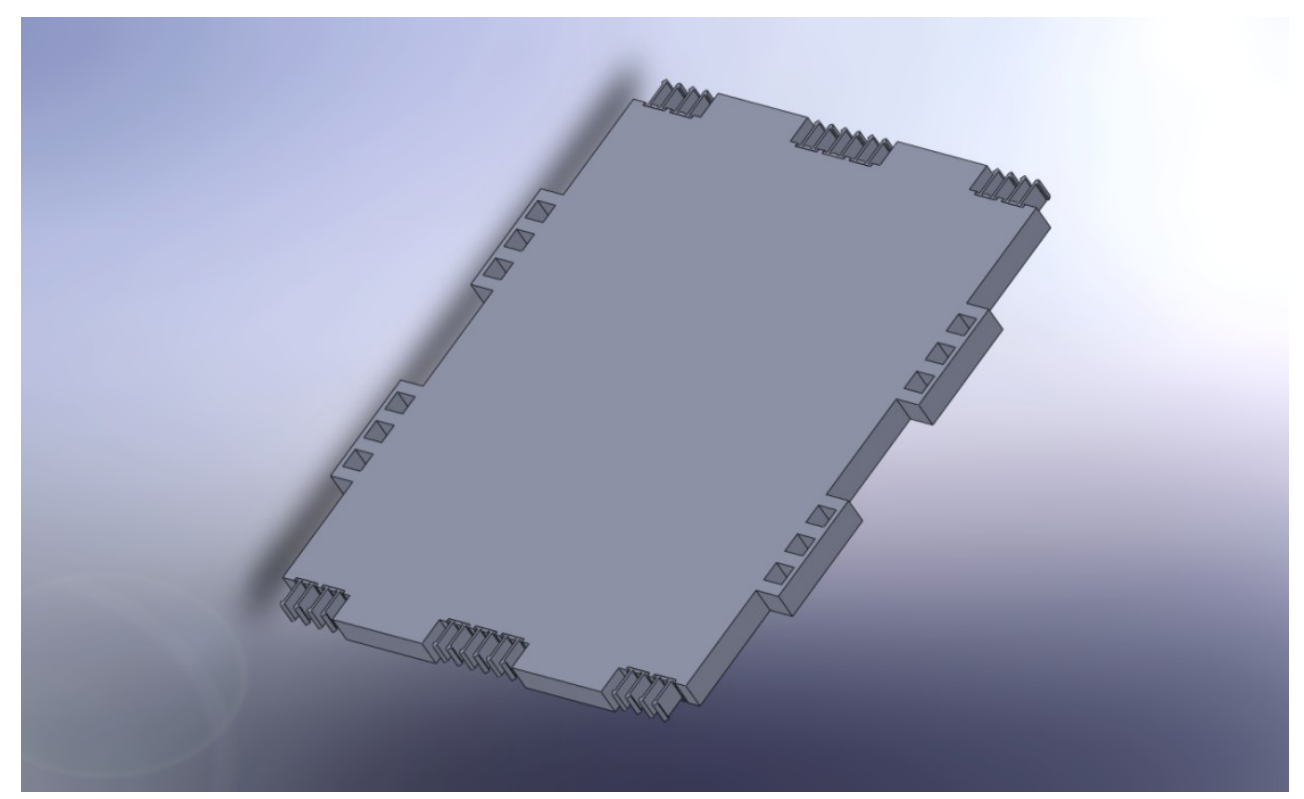

Figure 2.2: 3-D platform female component.

There are two important mechanical rules for creating a reliable spring-like silicon feature similar to the Hooks described above that are used in fixed-end cantilever beam designs: (i) length-to-height ratio should be less than ten; and (ii) displacement should be at most equal to the height value [67]. Since the proposed platform will use $250 \mu \mathrm{m}$ thick silicon wafers, the Hook length needs to be at least this value to fit properly through the Hole. Therefore, the Hook height and the total displacement should be less than $25 \mu \mathrm{m}$. Figure 2.3 is a close-up view of the Hook features found on the 3-D platform components. The Hook length is $258 \mu \mathrm{m}$, the height is $25 \mu \mathrm{m}$, the width is 200 $\mu \mathrm{m}$. The Hook indent dimension is an important consideration since it ultimately determines the amount of maximum displacement the Hook will undergo. The Hook indent dimension was chosen to be $20 \mu \mathrm{m}$ to keep the Hook displacement within the design rules yet be large enough to firmly snap and hold onto the female component when inserted completely through the Hole feature. Three different size Hole features 
were investigated in which all were $200 \mu \mathrm{m}$ high, $250 \mu \mathrm{m}$ deep and either $160 \mu \mathrm{m}, 170$ $\mu \mathrm{m}$, or $180 \mu \mathrm{m}$ wide as shown in Fig. 2.4. Notice the wider portion of the Hole on the wafer backside (50 $\mu \mathrm{m}$ deep) which is used for the Hook-end to snap onto.

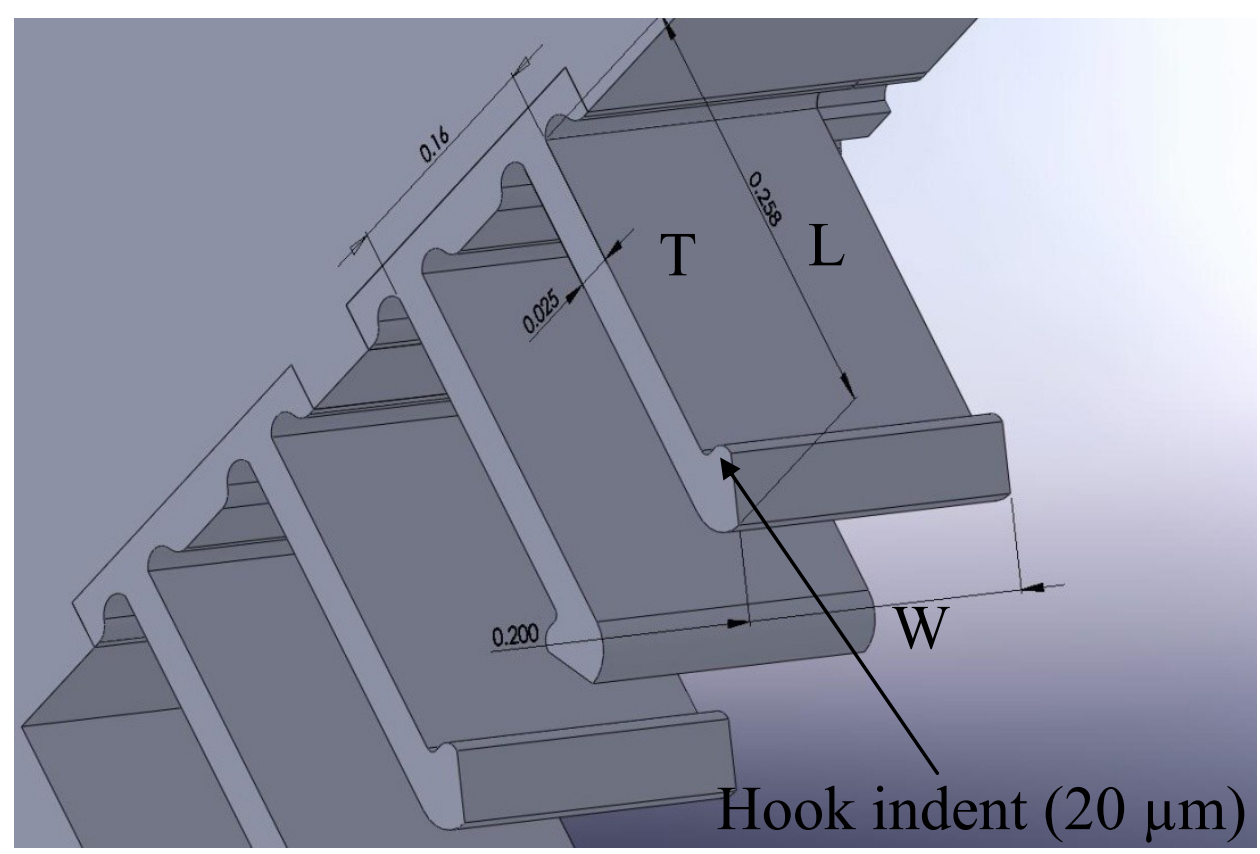

Figure 2.3: Close-up of "Hook" features (all units are in millimeters).

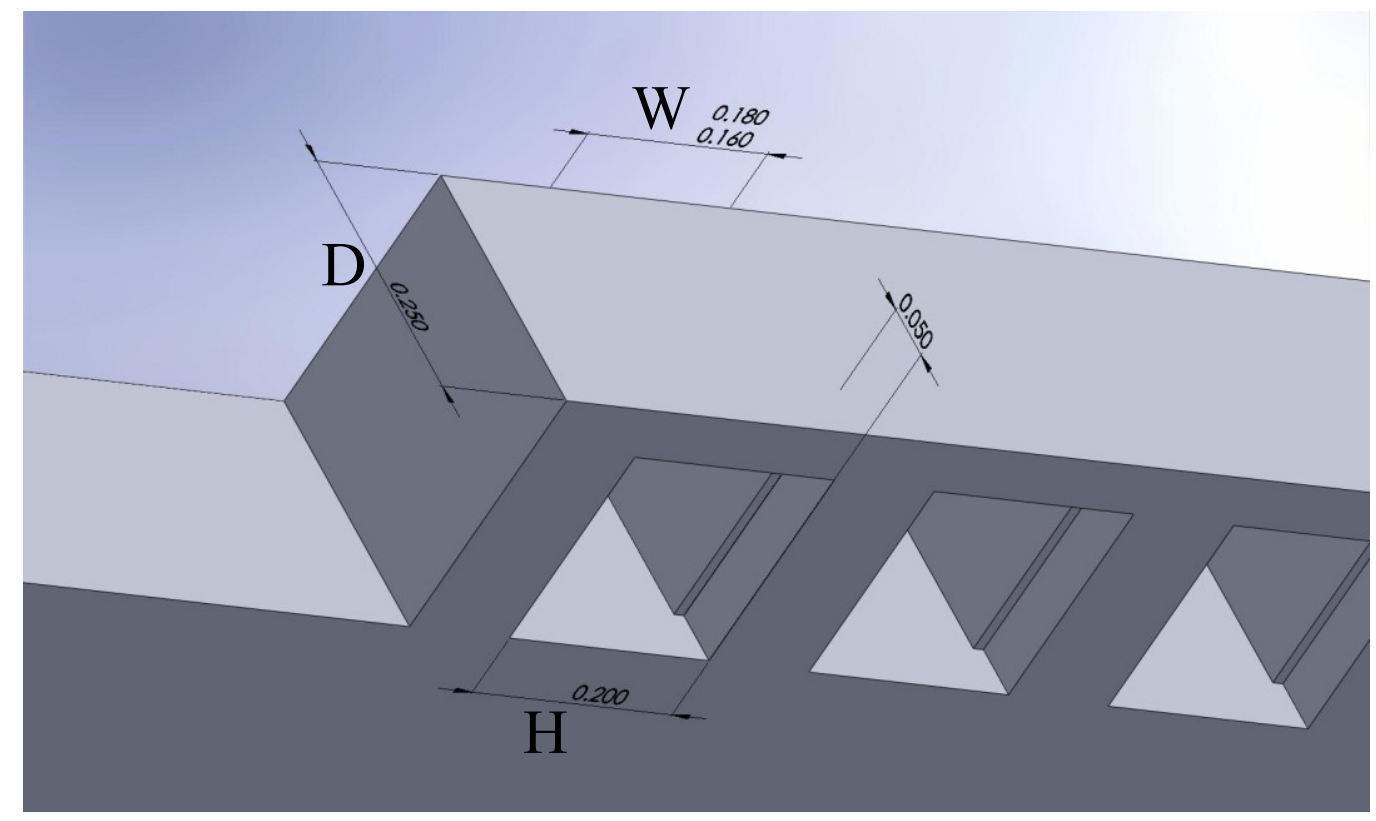

Figure 2.4: Close-up image of "Hole" features (all units are in millimeters). 
An entirely enclosed 3-D platform (i.e., with all components encapsulated on the inside) will require two male components and two female components as sidewalls, in addition to two end cap components as shown in Fig. 2.5, with thru-holes for future possible interconnections. Figure 2.6 shows all six component pieces just before assembly.

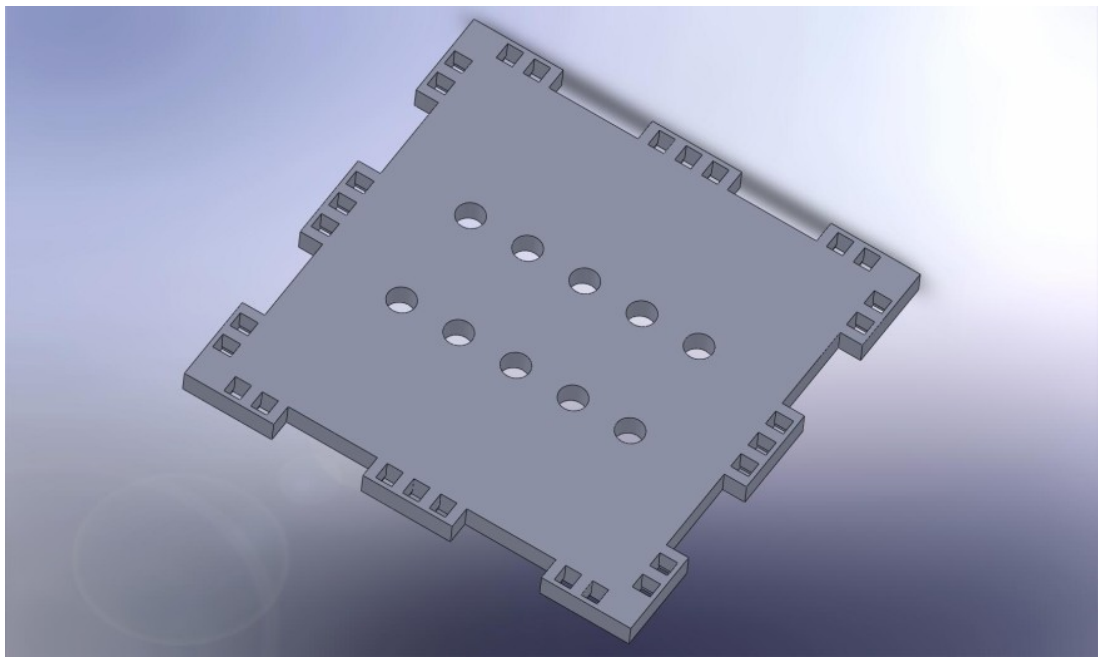

Figure 2.5: 3-D platform end cap component.

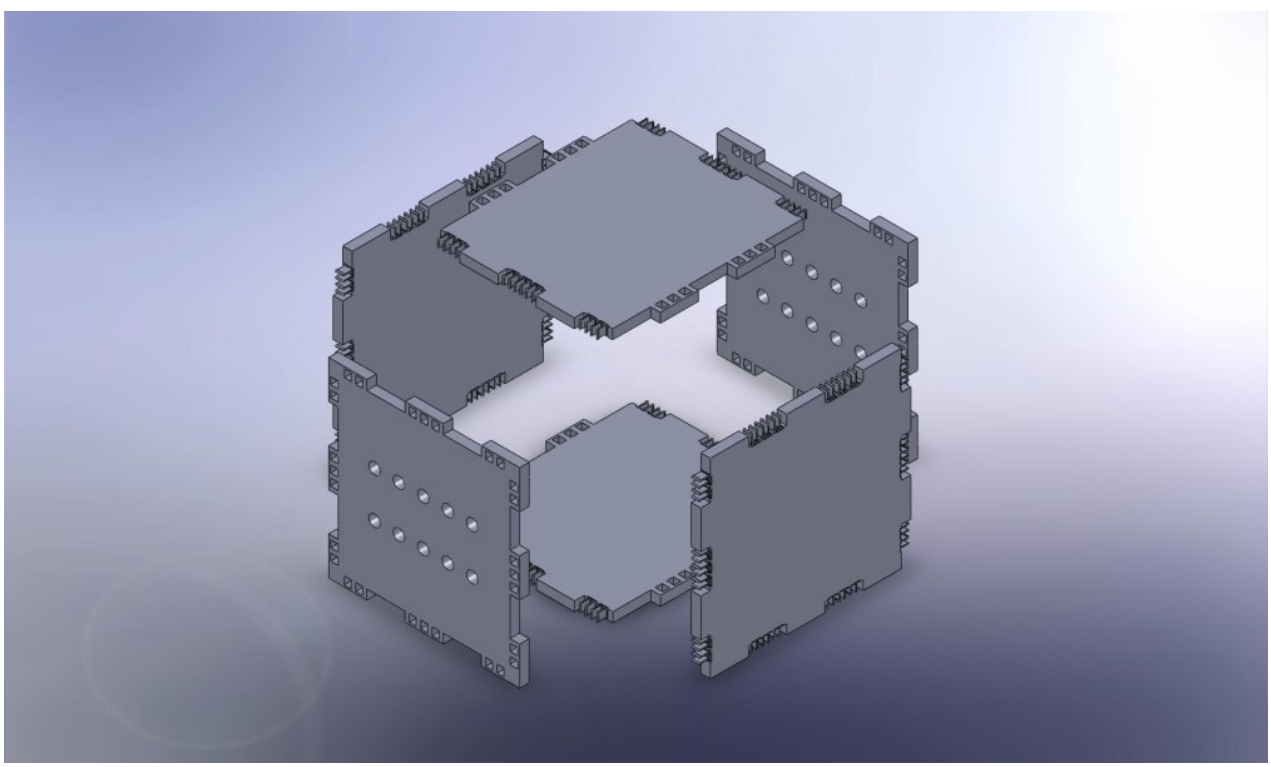

Figure 2.6: Complete 3-D platform components prior to assembly. 


\subsubsection{Finite-Element Modeling of Hook-Hole Features}

Using the COMSOL MultiPhysics ${ }^{\mathrm{TM}}$ finite element modeling software with the Structural Mechanics Module, stress-strain simulations of the hook-hole configurations were performed to optimize the Hook design and the female component Hole dimensions. For all the simulations, the default silicon material properties were used for both the Hook and Hole features. These properties and values are as follows: Young's Modulus, Isotropic (170GPa); Poisson's Ratio (0.28); density $\left(2329 \mathrm{~kg} / \mathrm{m}^{3}\right)$ and the Thermal Expansion Coefficient $\left(2.6 \times 10^{-6} 1 / \mathrm{K}\right)$ which are values typically used for $(100)$ type silicon wafers.

Two different Hook designs were investigated as shown in Fig. 2.7. The first design is defined as "non-flush" (Fig. 2.7(a)), as the curved portion at the base of the Hook would prevent the pieces from fitting flush together.

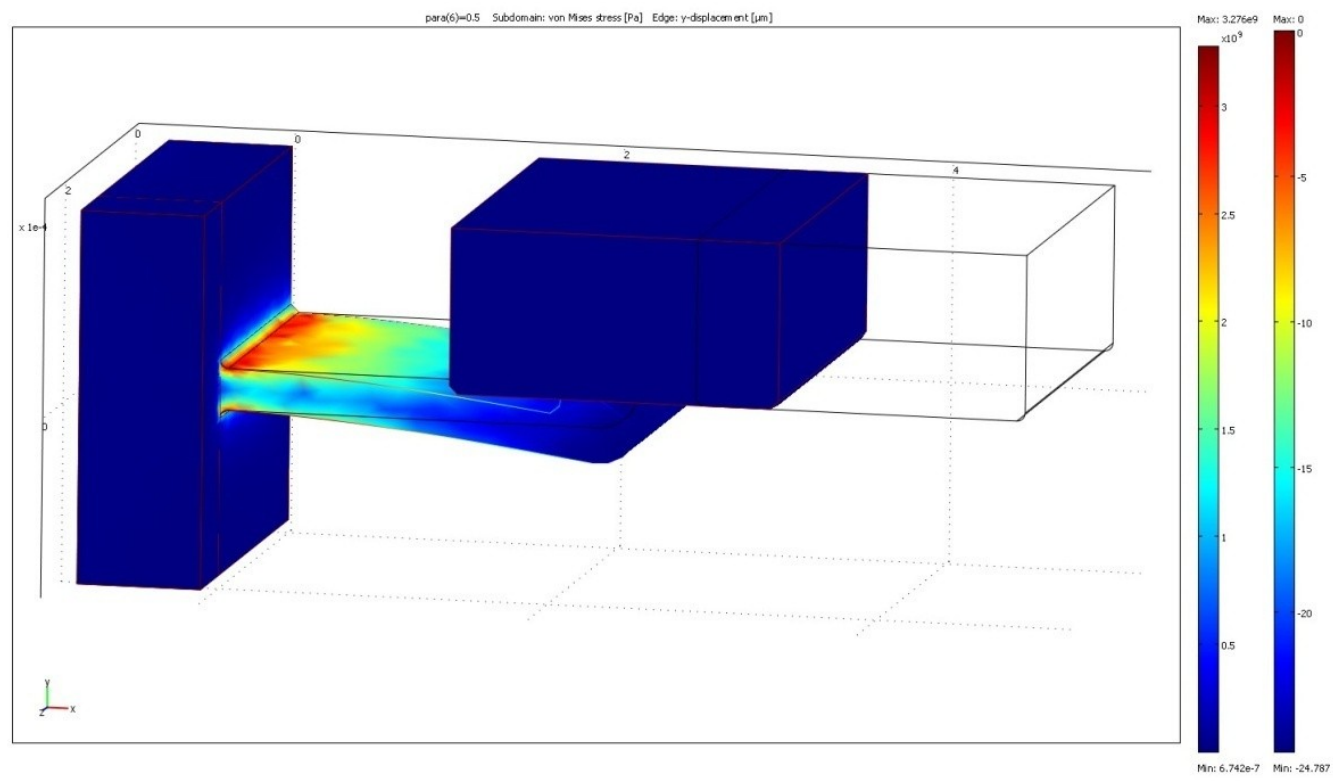

(a) 


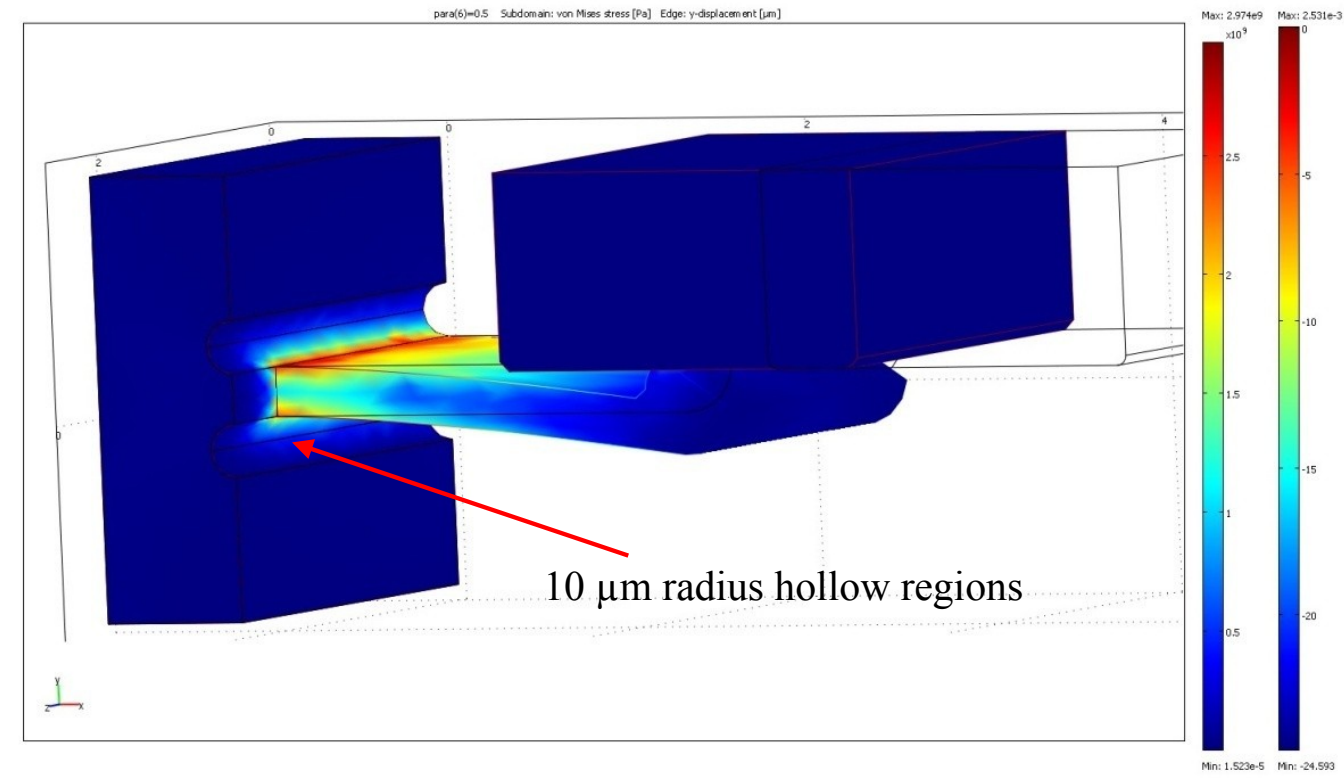

(b)

Figure 2.7: Hook designs: (a) non-flush; and (b) flush.

The second design (Fig. 2.7 (b) would allow for a "flush" fit between the mating pieces with the inclusion of hollowed out regions near the Hook's fixed end that have a radius of $10 \mu \mathrm{m}$. This would also slightly increase the Hook length which is advantageous to prevent the silicon from fracturing. Silicon wafers (100) have been shown to have a fracture strength of $2.8 \mathrm{GPa}$ [68]. Silicon micro-machined cantilever beams with similar dimensions to the Hook features have fracture strengths from $3.5 \mathrm{GPa}$ to $9.0 \mathrm{GPa}$ for uncoated wafers and 4.0 GPa to 7.0 GPa for wafers coated with a $0.53 \mu \mathrm{m}$ thick layer of silicon-dioxide [69]. For all the simulations here, a fracture is assumed to occur if any stress greater than $3.5 \mathrm{GPa}$ is found within the silicon structures. 
The 3-D stress-strain simulations are setup to apply an increasing vertical force from $200 \mathrm{~N} / \mathrm{m}$ to $2000 \mathrm{~N} / \mathrm{m}$ on the top edge of the Hooks for each design as shown in Fig. 2.8. The resulting maximum stress and Hook displacements are plotted in Fig. 2.9 along with a line indicating the fracture strength of $3.5 \mathrm{GPa}$.

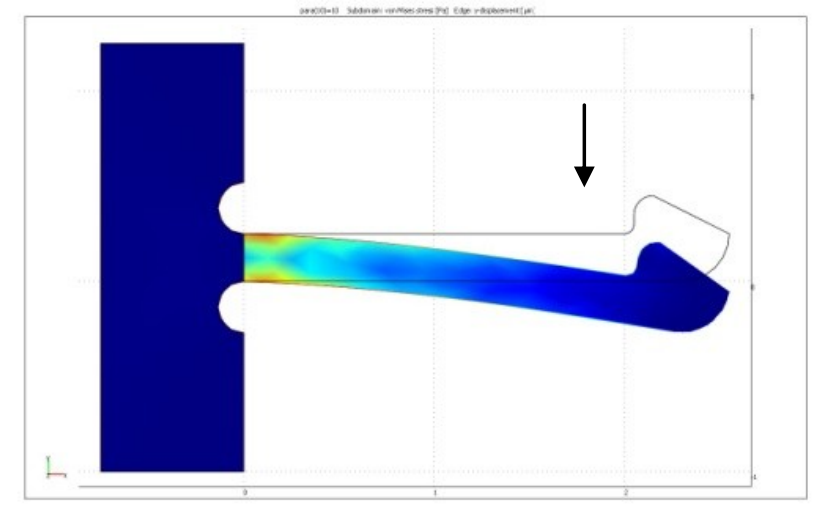

(a)

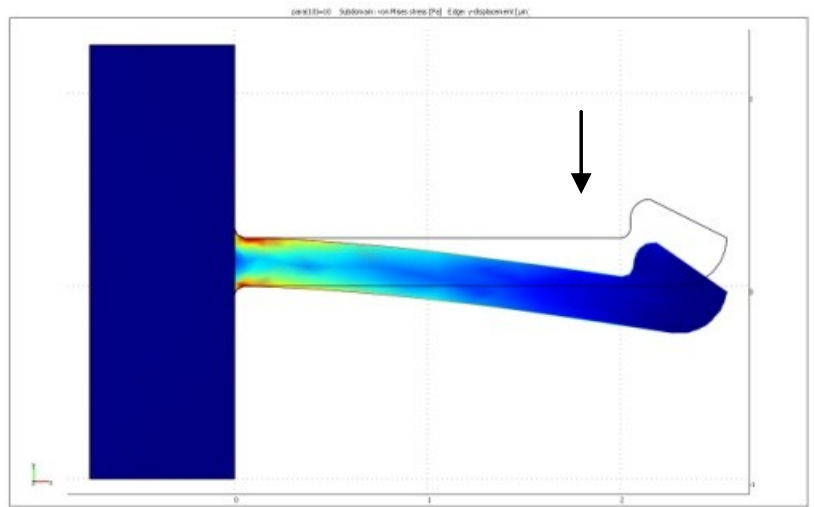

(b)

Figure 2.8: Side views of maximum stress and displacement with applied force to the Hook-end for the: (a) flush design; and (b) non-flush design. 

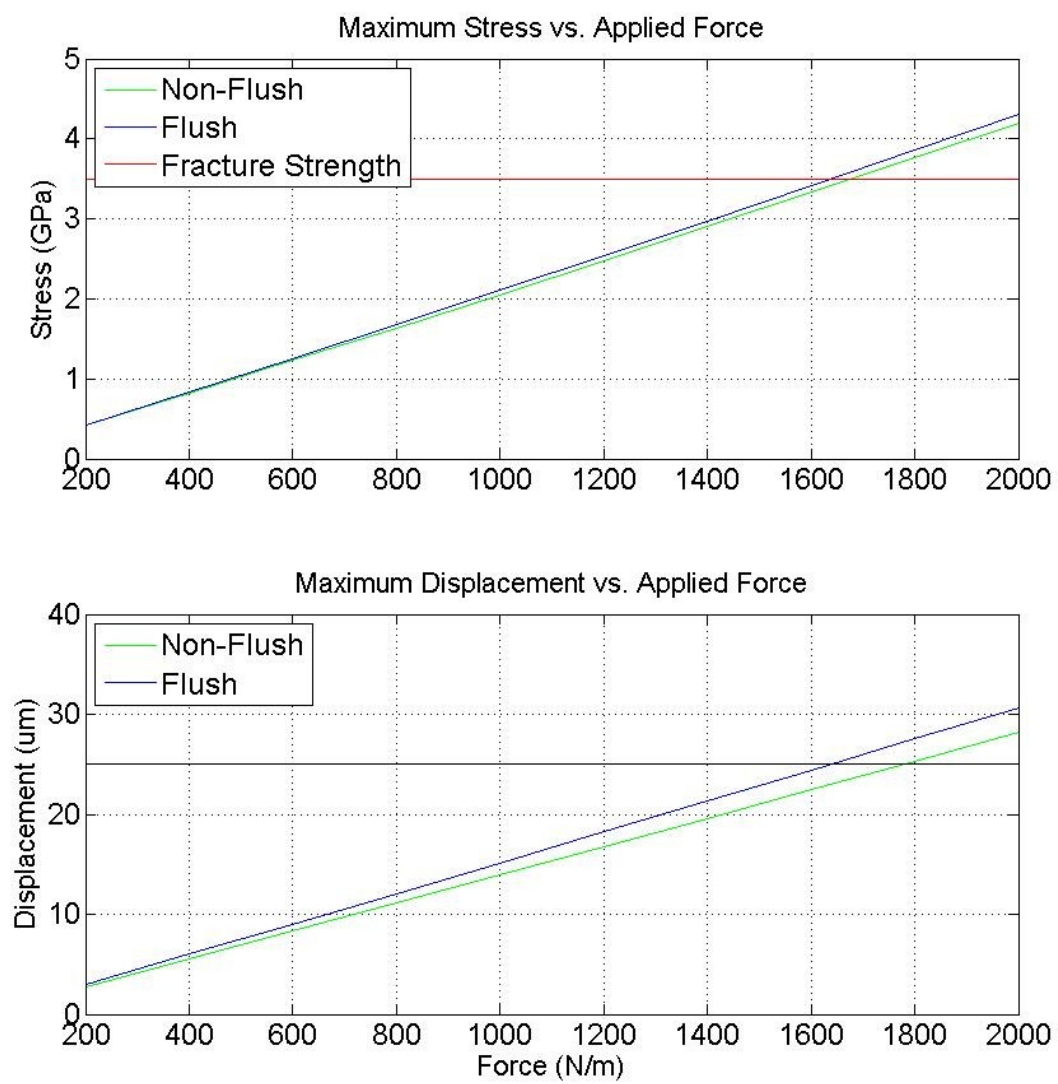

Figure 2.9: Simulation results for maximum stress (top) and maximum displacement (bottom) versus applied force for both flush and non-flush Hook structures.

From the simulation results in Fig. 2.9, the two designs have roughly the same maximum stress at a given applied force value and fracture is likely at $1600 \mathrm{~N} / \mathrm{m}$ where the maximum stress value approaches $3.5 \mathrm{GPa}$. However, the flush design has a greater displacement value than the non-flush design and is nearly at the maximum displacement of $25 \mu \mathrm{m}$ per the design rules for the Hook. A series of 3-D, dynamic finite element modeling simulations were performed with the non-flush and flush Hook designs using silicon Hole feature widths of $160 \mu \mathrm{m}, 170 \mu \mathrm{m}$ and $180 \mu \mathrm{m}$ as shown in Fig. 2.10. For each simulation, the Hole-feature was advanced towards the hook in $50 \mu \mathrm{m}$ increments 
and the maximum stress and displacement was found to be constant when the tip of the Hook indent feature was between points "A" and "B" in Fig. 2.10. The stress returned to zero once the tip of the Hook indent feature was inserted past point " $\mathrm{B}$ " and the Hook snap-locked into place.

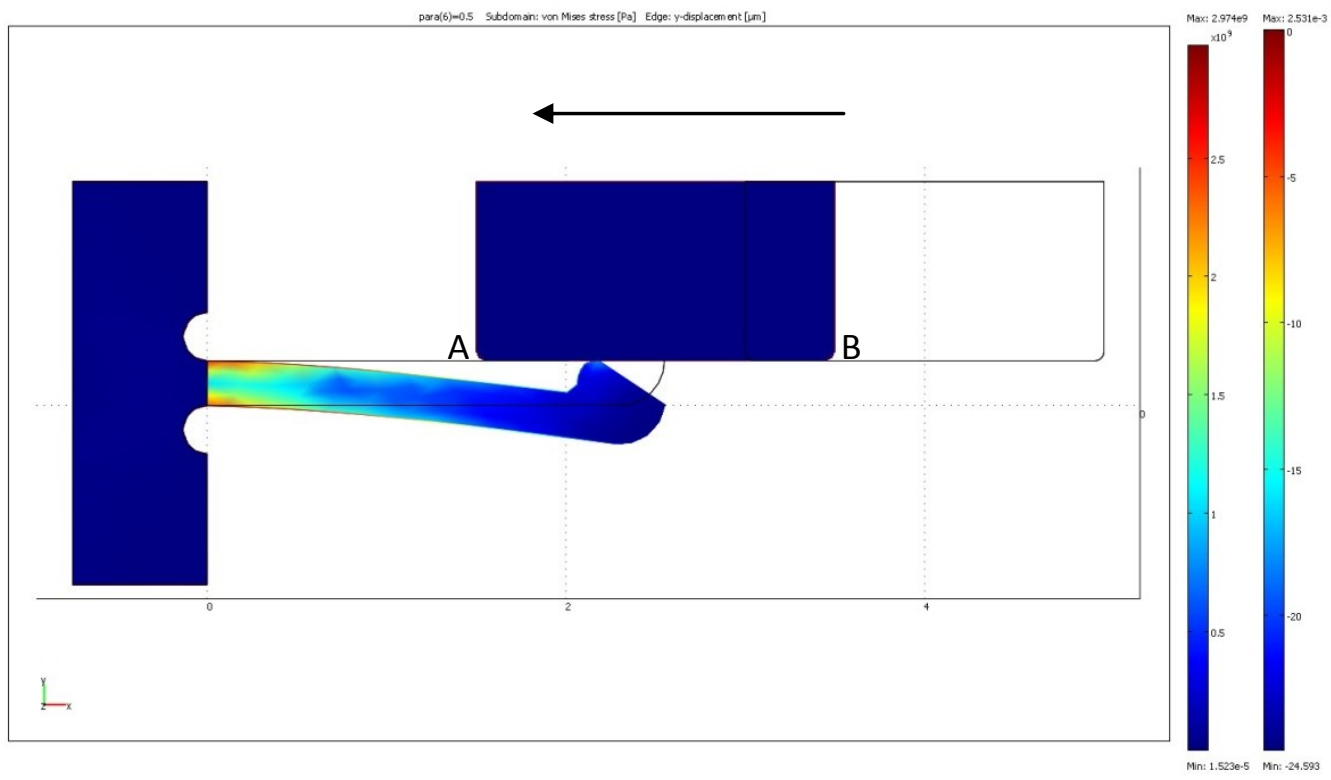

(a)

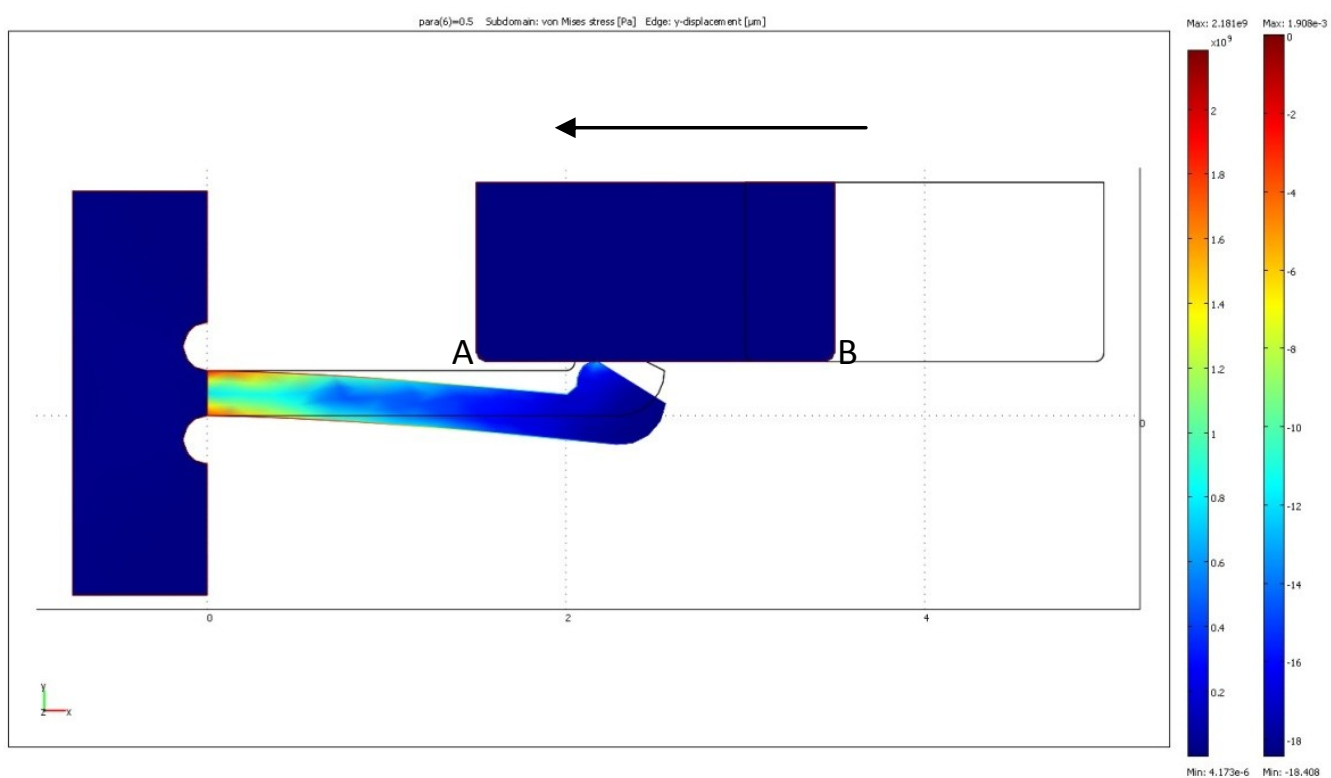

(b) 


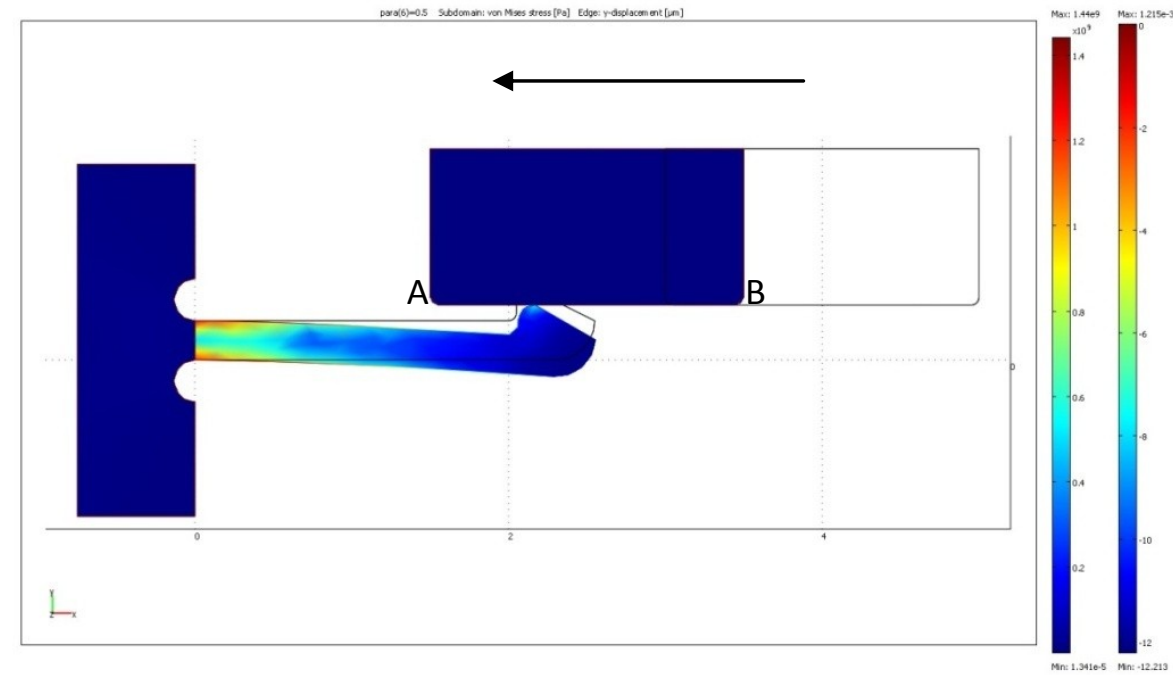

(c)

Figure 2.10: Dynamic 3-D stress-strain simulation results for flush Hooks and Holes with widths (a) $160 \mu \mathrm{m}$, (b) $170 \mu \mathrm{m}$ and (c) $180 \mu \mathrm{m}$. 
Figure 2.11 summarizes the resulting maximum stress and maximum displacements for all the dynamic, 3-D stress-strain simulations.
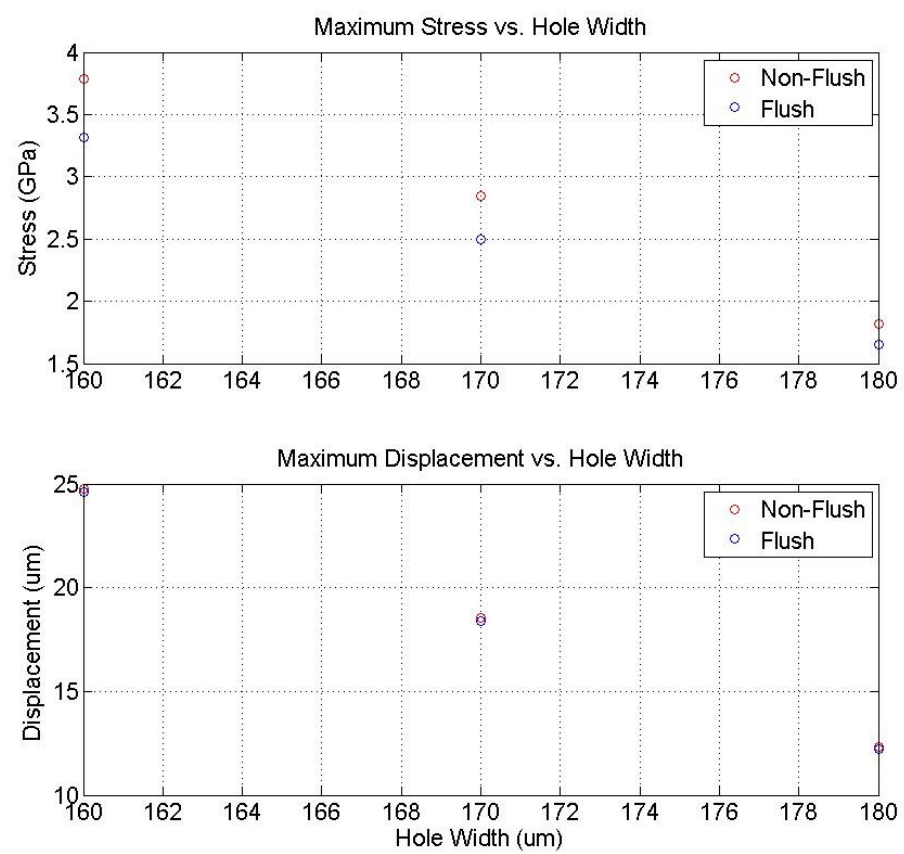

Figure 2.11: Results for dynamic, 3-D stress-strain simulation as a function of Hole width: (top) maximum stress; and (bottom) maximum displacement.

From Fig. 2.11, the different hook designs showed similar displacements for a given Hole-width as expected. Also, increasing the Hole-width greatly reduced the amount of stress found within the silicon Hook features by half when increasing the Hole-width from $160 \mu \mathrm{m}$ to $180 \mu \mathrm{m}$. Based on these dynamic 3-D Hook-Hole simulation results, the flush Hook-design and an $180 \mu \mathrm{m}$ Hole-width was selected for all the periphery features on the male, female and end cap platform components. Increasing the Hole width greater than $180 \mu \mathrm{m}$ was not considered because the silicon material between Hole features would begin to get too thin $(<120 \mu \mathrm{m})$ increasing the chances of damaging the platform component pieces during fabrication and assembly. 


\subsection{Connection Design Using Interdigitated Teeth (i.e., No Hook-Hole Features)}

A second type of platform connector is investigated which uses interdigitated teeth around the periphery of the platform component segments. Although, these features cannot hold individual pieces together like the Hook-Hole method above, the interdigitated teeth simplifies the microfabrication process by eliminating one photomask step and allowing greater flexibility during the assembly process.

\subsubsection{Specifications for Three Different Configurations}

The interdigitated teeth design closely resembles the Hook-Hole design except the Hole features are filled in and the Hook features are removed and only straight, orthogonal features are used to mate adjacent platform pieces as shown in Fig. 2. 12.

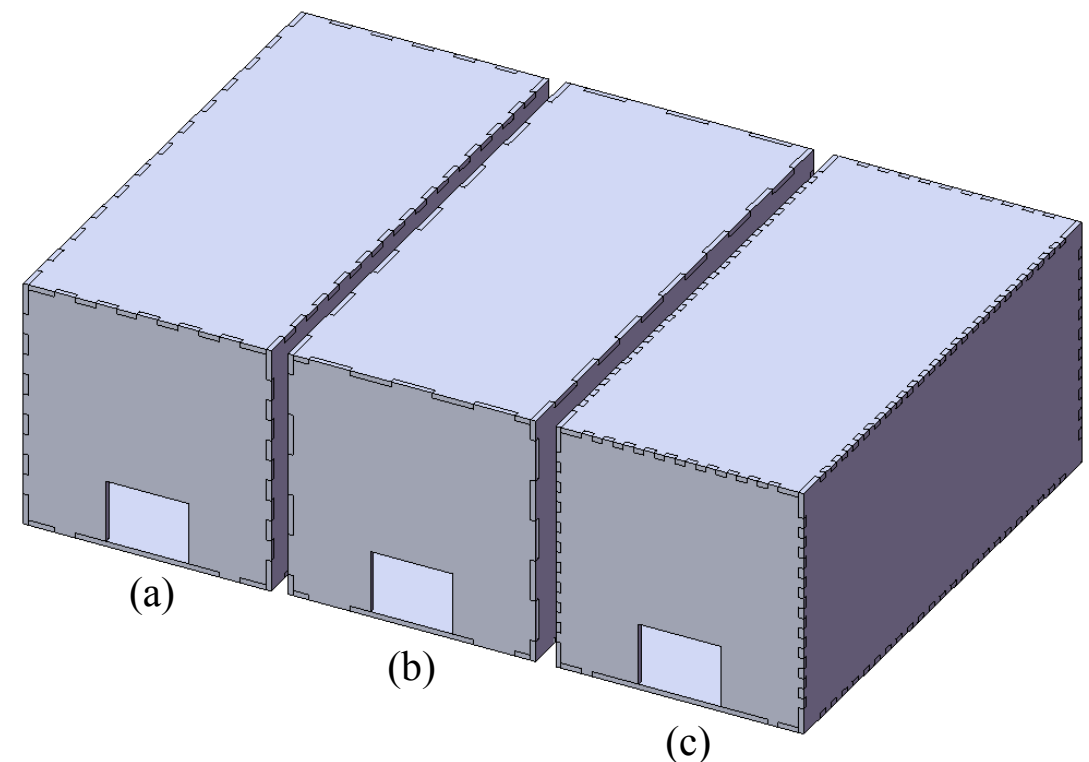

Figure 2.12: Three interdigitated teeth designs: (a) $1 \mathrm{~mm}$ teeth length; (b) $2 \mathrm{~mm}$ teeth length; and (c) $0.5 \mathrm{~mm}$ teeth length. 
For each of the three teeth designs, the maximum number of teeth were evenly placed on each platform edge and the teeth spacing was two times the teeth length. All three platform structures shown in Fig. 2.12 measure $12 \times 12 \times 25 \mathrm{~mm}^{3}$ and are used in the following section which investigates the platform mechanical strength for the teeth designs.

\subsubsection{Description of Finite-Element Modeling Simulation Conditions}

In this section and throughout this chapter, 3-D finite-element modeling (FEM) simulations are performed for the following conditions exerted on the platform: pressure $(\mathrm{Pa})$, edge force $(\mathrm{N} / \mathrm{m})$ and point forces $(\mathrm{N})$. The following defines the terminology and boundary conditions for each of these simulation scenarios. Six different simulation configurations for pressure and point force simulations are depicted in Fig. 2.13.

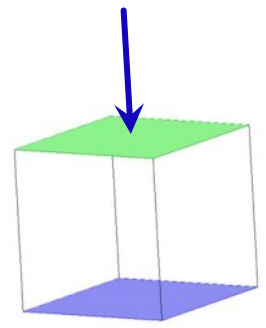

(a)

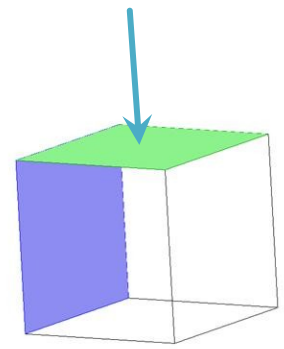

(d)

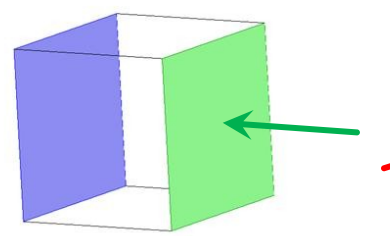

(b)

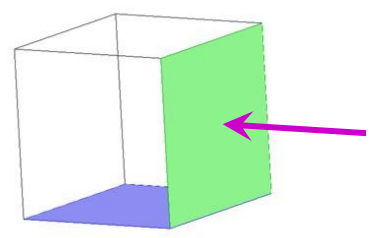

(e)

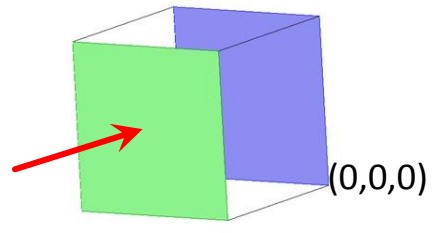

(c)

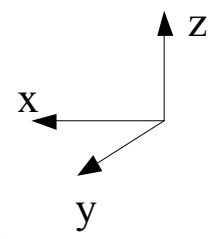

Figure 2.13: Pressure and point force simulation configurations where the blue surface is fixed and the green surface has the applied force: (a) Top Compression; (b) Side Compression; (c) Endcap Compression; (d) Top Shear; (e) Side Shear; and (f) End cap Shear. 
For the pressure simulations, the pressure was applied in the normal direction, evenly across the platform face and varied from $0.4 \mathrm{MPa}$ to $8.0 \mathrm{MPa}$. The point force simulations were also applied in the normal direction at a point directly in the center of the platform face and varied from $6 \mathrm{~N}$ to $120 \mathrm{~N}$

For the edge force simulations, the force was applied at an angle of $45^{\circ}$ with respect to the surface normal direction, evenly across the platform edge and varied from $2,000 \mathrm{~N} / \mathrm{m}$ to $40,000 \mathrm{~N} / \mathrm{m}$. Figure 2.13 illustrates the six edge force simulation configurations. For all simulations the following silicon material properties were used: Young's Modulus $=186.5 \mathrm{GPa}$; Poisson's Ratio $=0.26$; and the density $=2,329 \mathrm{~kg} / \mathrm{m}^{3}$.

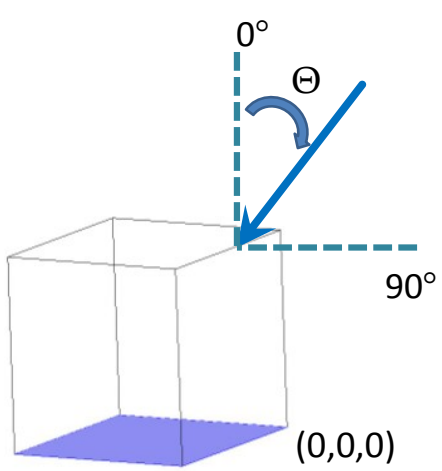

(a)

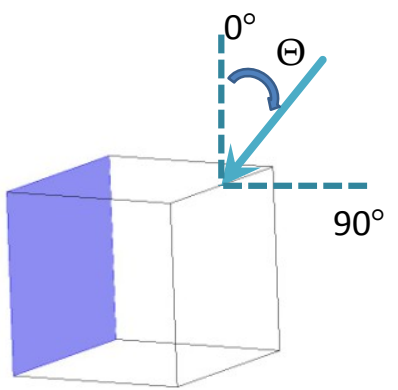

(d)

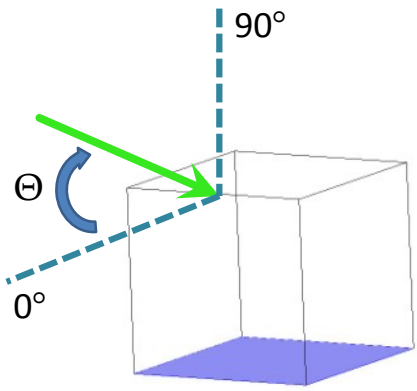

(b)

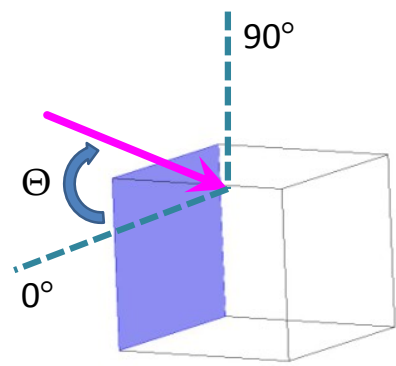

(e)

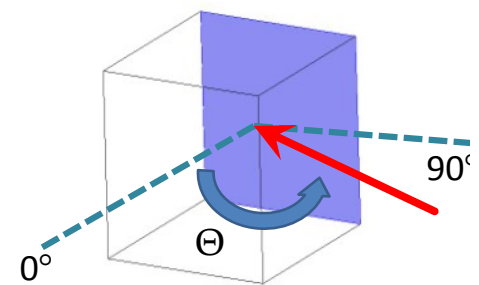

(c)
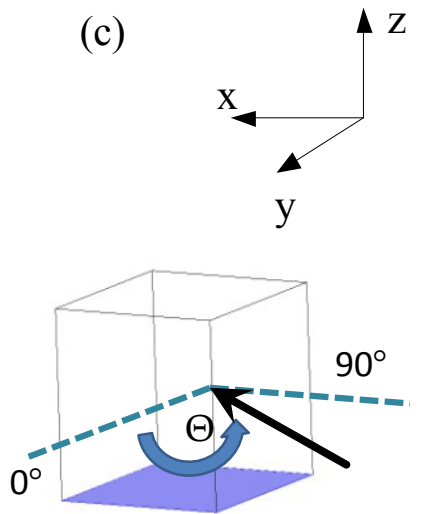

(f)

Figure 2.14: Edge force simulation configurations where the blue surface is fixed: (a) Top-Side Edge Compression; (b) Top-Front Edge Compression; (c) Side-Front Edge Compression; (d) Top-Side Edge Shear; (e) Top-Front Edge Shear; (f) Side-Front Edge Shear. 
These values represent a (111) oriented silicon wafer. In Figs. 2.13 and 2.14, the front face of the platform is always the end cap/connector component piece. Also, in Figs. 2.13 and 2.14, the coordinate axes and origin location are shown and remains constant throughout all the presented results. All other portions of the platform not explicitly described above are free to move in any direction during the simulations.

\subsubsection{Finite-Element Modeling Results}

Figures $2.15-2.23$ show the simulation results and Tables $2.1-2.9$ contain the best fit line equation using the MatLab ${ }^{\circledR}$ polyfit() function, the maximum stress location and the maximum input pressure assuming a $3.5 \mathrm{GPa}$ fracture strength.

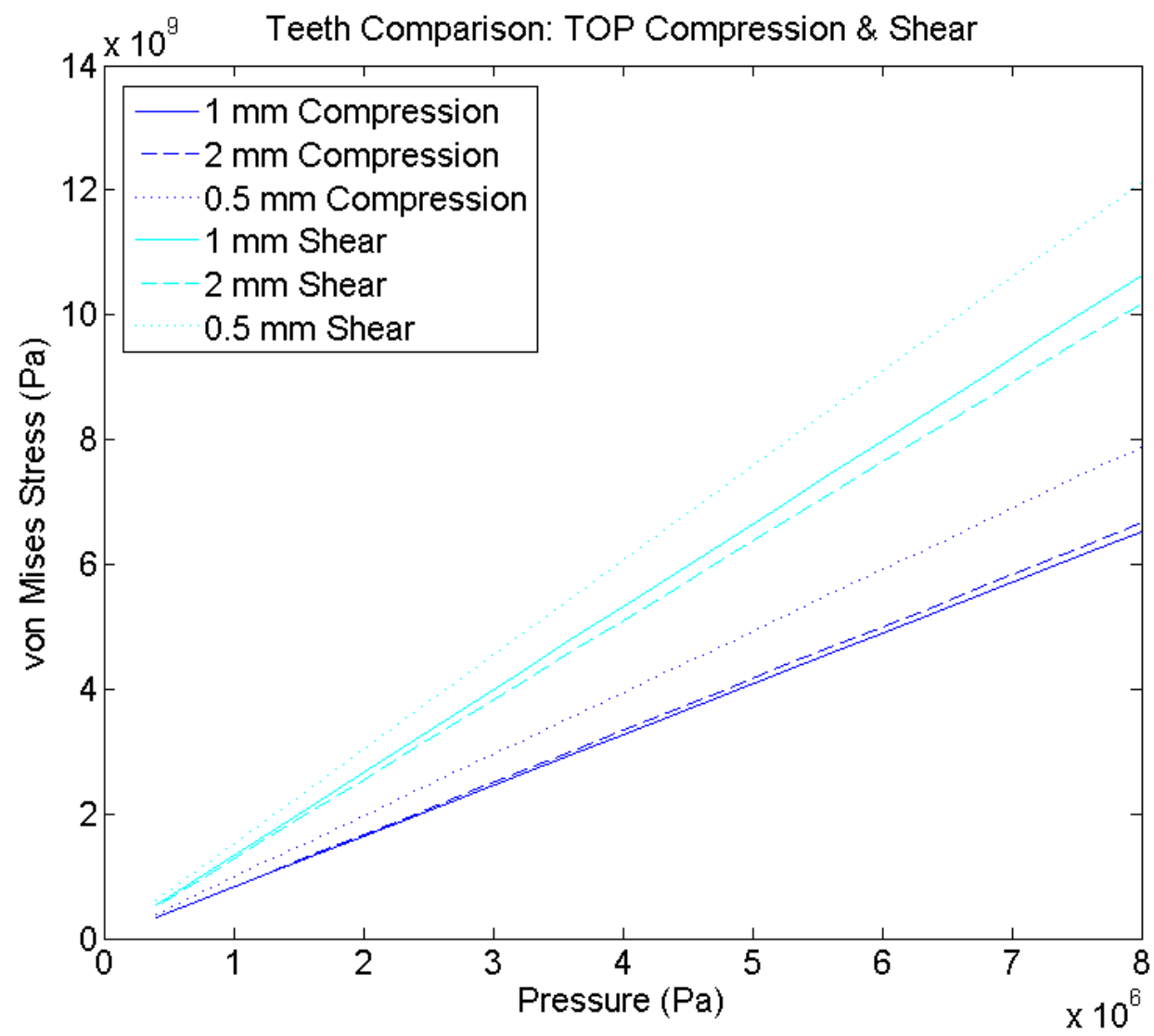

Figure 2.15: Top side compression and shear pressure simulation results for the three teeth designs. 


\begin{tabular}{|r|c|c|c|}
\hline \multicolumn{1}{l|}{$\begin{array}{l}\text { Top } \\
\text { Compression }\end{array}$} & $\begin{array}{c}\text { Best-Fit Linear } \\
\text { Equation }(\mathrm{Pa})\end{array}$ & $\begin{array}{c}\text { Max. Input } \\
\text { Pressure }(\mathrm{MPa})\end{array}$ & $\begin{array}{c}\text { Failure location } \\
(\mathrm{x}, \mathrm{y}, \mathrm{z}) \mathrm{mm}\end{array}$ \\
\hline $1 \mathrm{~mm}$ teeth & $\mathrm{Y}=(814.73) \mathrm{X}$ & $\mathrm{X}=4.30$ & $(0.125,10.1,12.0)$ \\
\hline $0.5 \mathrm{~mm}$ teeth & $\mathrm{Y}=(985.98) \mathrm{X}$ & $\mathrm{X}=3.55$ & $(0.125,14.2,11.9)$ \\
\hline $2 \mathrm{~mm}$ teeth & $\mathrm{Y}=(833.24) \mathrm{X}$ & $\mathrm{X}=4.20$ & $(0.0,9.3,11.8)$ \\
\hline Top Shear & & & \\
\hline $1 \mathrm{~mm}$ teeth & $\mathrm{Y}=(1329.1) \mathrm{X}$ & $\mathrm{X}=2.63$ & $(11.8,12.9,11.7)$ \\
\hline $0.5 \mathrm{~mm}$ teeth & $\mathrm{Y}=(1515.5) \mathrm{X}$ & $\mathrm{X}=2.31$ & $(11.7,10.2,11.8)$ \\
\hline $2 \mathrm{~mm}$ teeth & $\mathrm{Y}=(1273.6) \mathrm{X}$ & $\mathrm{X}=2.75$ & $(11.5,14.2,12.0)$ \\
\hline
\end{tabular}

Table 2.1: Top compression and shear pressure simulation results for the three teeth designs.

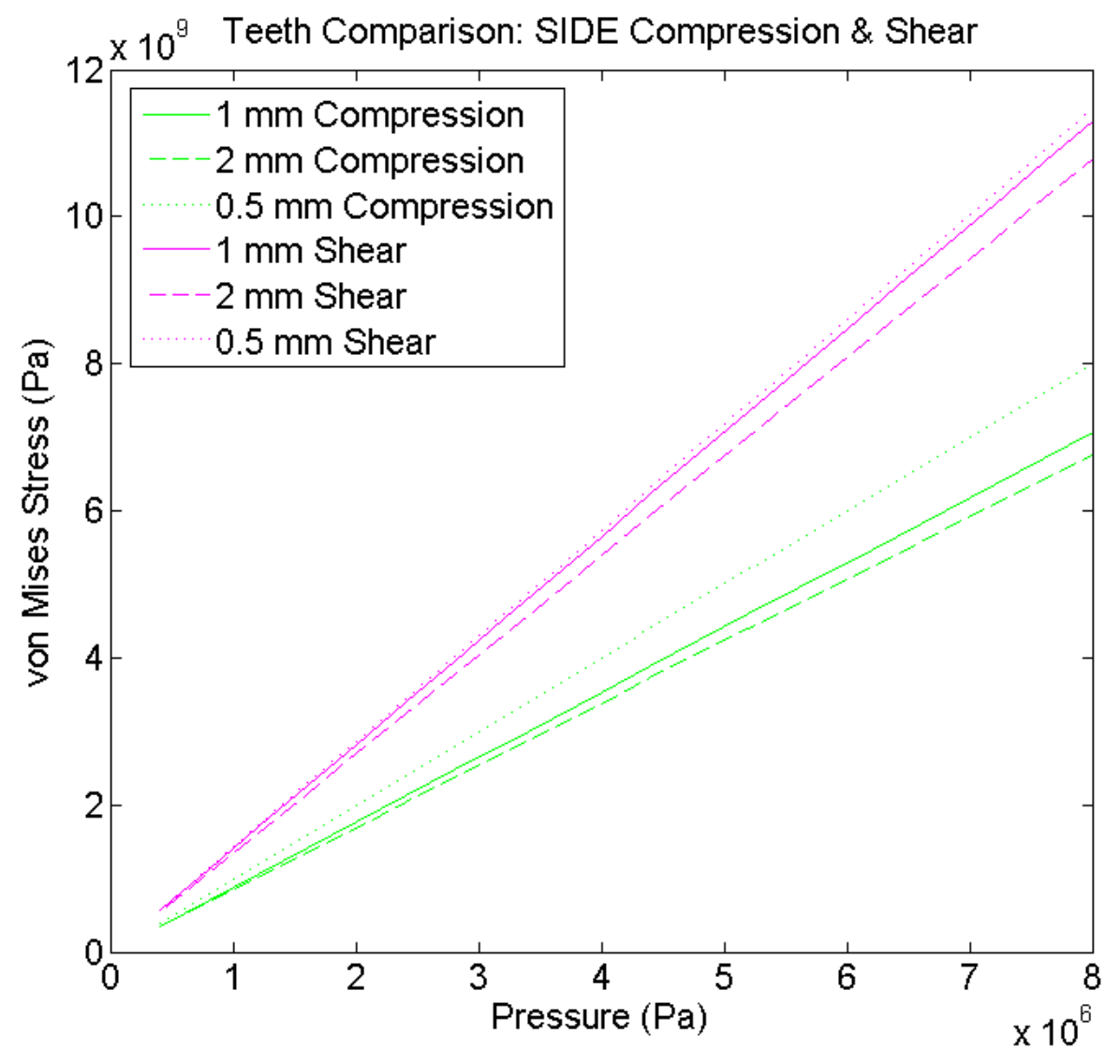

Figure 2.16: Side compression and shear pressure simulation results for the three teeth designs. 


\begin{tabular}{|r|c|c|c|}
\hline \multicolumn{1}{l}{$\begin{array}{l}\text { Side } \\
\text { Compression }\end{array}$} & $\begin{array}{c}\text { Best-Fit Linear } \\
\text { Equation }(\mathrm{Pa})\end{array}$ & $\begin{array}{c}\text { Max. Input } \\
\text { Pressure }(\mathrm{MPa})\end{array}$ & $\begin{array}{c}\text { Failure location } \\
(\mathrm{x}, \mathrm{y}, \mathrm{z}) \mathrm{mm}\end{array}$ \\
\hline $1 \mathrm{~mm}$ teeth & $\mathrm{Y}=(882.76) \mathrm{X}$ & $\mathrm{X}=3.96$ & $(0.125,10.1,12.0)$ \\
\hline $0.5 \mathrm{~mm}$ teeth & $\mathrm{Y}=(1001.4) \mathrm{X}$ & $\mathrm{X}=3.50$ & $(0.125,10.9,0.0)$ \\
\hline $2 \mathrm{~mm}$ teeth & $\mathrm{Y}=(845.22) \mathrm{X}$ & $\mathrm{X}=4.14$ & $(0,6.1,12.0)$ \\
\hline Side Shear & & & \\
\hline $1 \mathrm{~mm}$ teeth & $\mathrm{Y}=(1412.5) \mathrm{X}$ & $\mathrm{X}=2.48$ & $(0.25,9.9,0.25)$ \\
\hline $0.5 \mathrm{~mm}$ teeth & $\mathrm{Y}=(1433.6) \mathrm{X}$ & $\mathrm{X}=2.44$ & $(0.25,10.7,0.25)$ \\
\hline $2 \mathrm{~mm}$ teeth & $\mathrm{Y}=(1347.7) \mathrm{X}$ & $\mathrm{X}=2.60$ & $(0.25,15.8,0.25)$ \\
\hline
\end{tabular}

Table 2.2: Side compression and shear pressure simulation results for the three teeth designs.

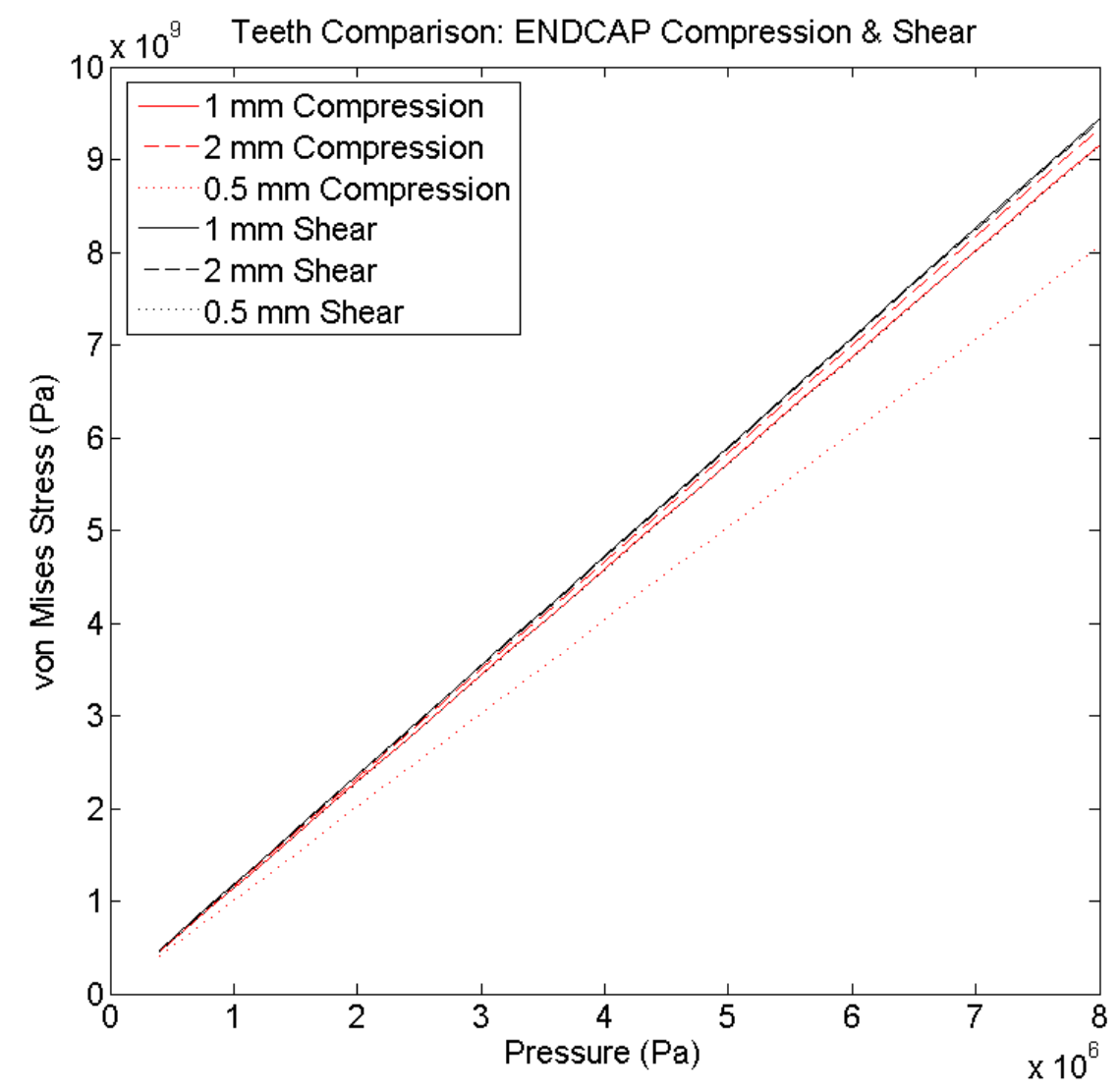

Figure 2.17: End cap compression and shear pressure simulation results for the three teeth designs. 


\begin{tabular}{|r|c|c|c|}
\hline $\begin{array}{l}\text { End cap } \\
\text { Compression }\end{array}$ & $\begin{array}{c}\text { Best-Fit Linear } \\
\text { Equation }(\mathrm{Pa})\end{array}$ & $\begin{array}{c}\text { Max. Input } \\
\text { Pressure }(\mathrm{MPa})\end{array}$ & $\begin{array}{c}\text { Failure location } \\
(\mathrm{x}, \mathrm{y}, \mathrm{z}) \mathrm{mm}\end{array}$ \\
\hline $1 \mathrm{~mm}$ teeth & $\mathrm{Y}=(1145.9) \mathrm{X}$ & $\mathrm{X}=3.05$ & $(8.0,25.3,0.0)$ \\
\hline $0.5 \mathrm{~mm}$ teeth & $\mathrm{Y}=(1008.6) \mathrm{X}$ & $\mathrm{X}=3.47$ & $(8.0,25.5,0.25)$ \\
\hline $2 \mathrm{~mm}$ teeth & $\mathrm{Y}=(1166.7) \mathrm{X}$ & $\mathrm{X}=3.00$ & $(4.0,25.5,0.25)$ \\
\hline End cap Shear & & & \\
\hline $1 \mathrm{~mm}$ teeth & $\mathrm{Y}=(1180.3) \mathrm{X}$ & $\mathrm{X}=2.97$ & $(8.0,25.3,0.25)$ \\
\hline $0.5 \mathrm{~mm}$ teeth & $\mathrm{Y}=(1143.9) \mathrm{X}$ & $\mathrm{X}=3.06$ & $(8.0,25.5,0.25)$ \\
\hline $2 \mathrm{~mm}$ teeth & $\mathrm{Y}=(1177.7) \mathrm{X}$ & $\mathrm{X}=2.97$ & $(4.0,25.3,0.25)$ \\
\hline
\end{tabular}

Table 2.3: End cap compression and shear pressure simulation results for the three teeth designs.

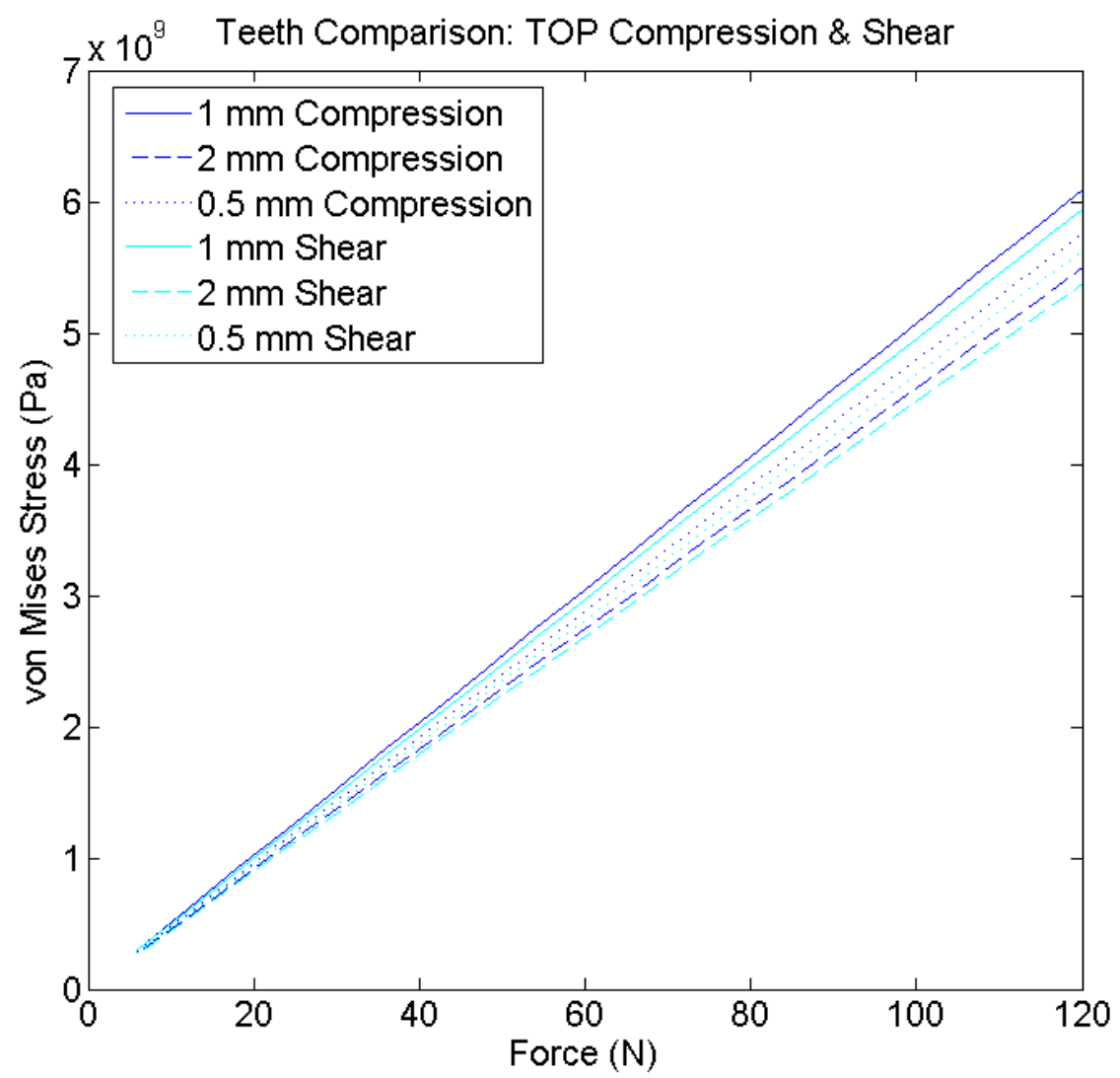

Figure 2.18: Top compression and shear point force simulation results for the three teeth designs. 


\begin{tabular}{|r|c|c|c|}
\hline \multicolumn{1}{l|}{$\begin{array}{l}\text { Top } \\
\text { Compression }\end{array}$} & $\begin{array}{c}\text { Best-Fit Linear } \\
\text { Equation }(\mathrm{Pa})\end{array}$ & $\begin{array}{c}\text { Max. Input } \\
\text { Force }(\mathrm{N})\end{array}$ & $\begin{array}{c}\text { Failure location } \\
(\mathrm{x}, \mathrm{y}, \mathrm{z}) \mathrm{mm}\end{array}$ \\
\hline $1 \mathrm{~mm}$ teeth & $\mathrm{Y}=\left(5.08 \times 10^{7}\right) \mathrm{X}$ & $\mathrm{X}=68.90$ & $(6.0,12.5,12.0)$ \\
\hline $0.5 \mathrm{~mm}$ teeth & $\mathrm{Y}=\left(4.80 \times 10^{7}\right) \mathrm{X}$ & $\mathrm{X}=72.92$ & $(6.0,12.7,12.0)$ \\
\hline $2 \mathrm{~mm}$ teeth & $\mathrm{Y}=\left(4.58 \times 10^{7}\right) \mathrm{X}$ & $\mathrm{X}=76.42$ & $(6.0,12.8,12.0)$ \\
\hline Top Shear & & & \\
\hline $1 \mathrm{~mm}$ teeth & $\mathrm{Y}=\left(4.96 \times 10^{7}\right) \mathrm{X}$ & $\mathrm{X}=70.56$ & $(6.0,12.5,12.0)$ \\
\hline $0.5 \mathrm{~mm}$ teeth & $\mathrm{Y}=\left(4.69 \times 10^{7}\right) \mathrm{X}$ & $\mathrm{X}=74.63$ & $(6.0,12.7,12.0)$ \\
\hline $2 \mathrm{~mm}$ teeth & $\mathrm{Y}=\left(4.48 \times 10^{7}\right) \mathrm{X}$ & $\mathrm{X}=78.13$ & $(6.0,12.8,12.0)$ \\
\hline
\end{tabular}

Table 2.4: Top compression and shear point force simulation results for the three teeth designs.

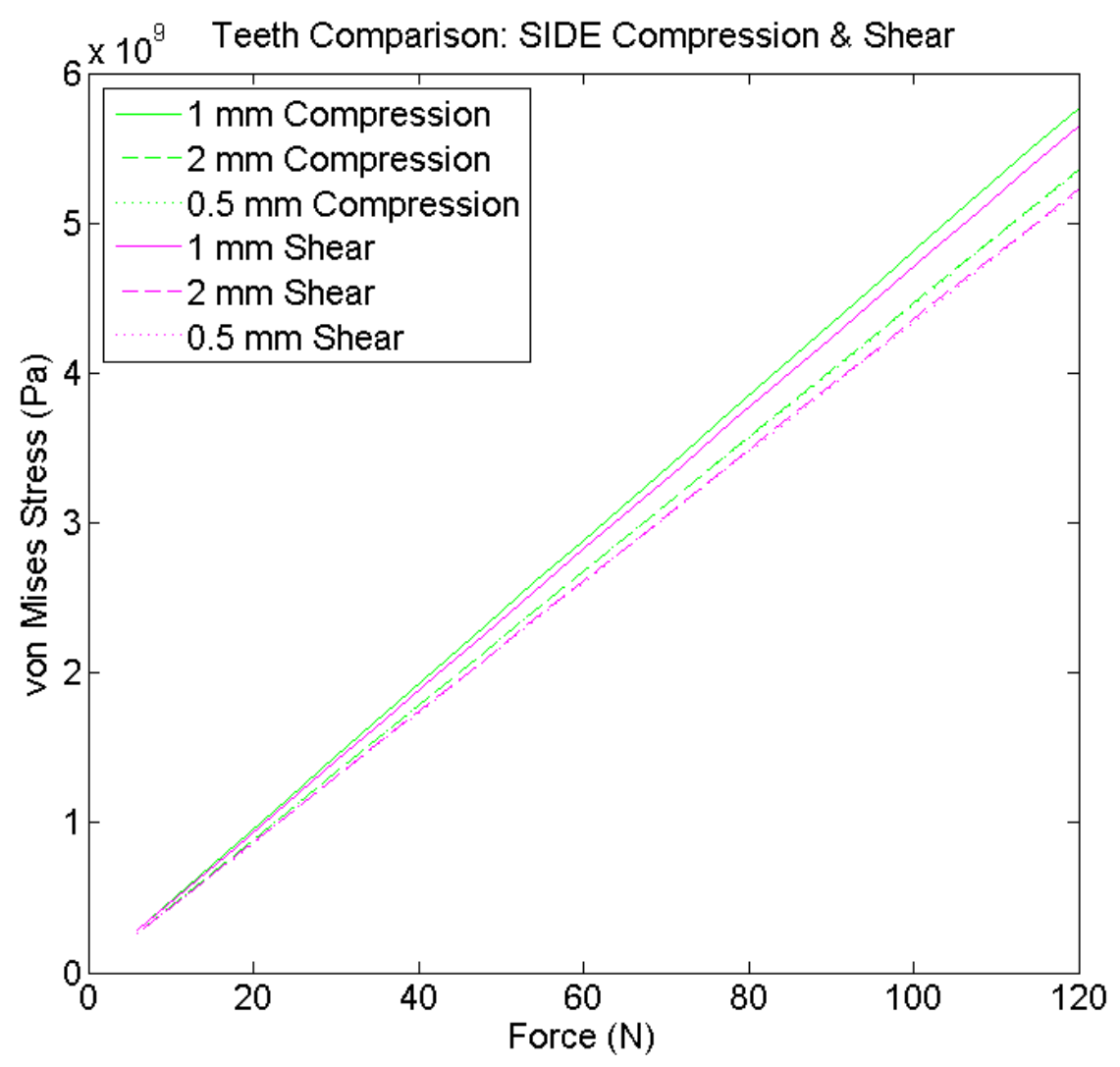

Figure 2.19: Side compression and shear point force simulation results for the three teeth designs. 


\begin{tabular}{|r|c|c|c|}
\hline \multicolumn{1}{l}{$\begin{array}{l}\text { Side } \\
\text { Compression }\end{array}$} & $\begin{array}{c}\text { Best-Fit Linear } \\
\text { Equation }(\mathrm{Pa})\end{array}$ & $\begin{array}{c}\text { Max. Input } \\
\text { Force }(\mathrm{N})\end{array}$ & $\begin{array}{c}\text { Failure location } \\
(\mathrm{x}, \mathrm{y}, \mathrm{z}) \mathrm{mm}\end{array}$ \\
\hline $1 \mathrm{~mm}$ teeth & $\mathrm{Y}=\left(4.81 \times 10^{7}\right) \mathrm{X}$ & $\mathrm{X}=72.77$ & $(0.0,12.5,6.0)$ \\
\hline $0.5 \mathrm{~mm}$ teeth & $\mathrm{Y}=\left(4.46 \times 10^{7}\right) \mathrm{X}$ & $\mathrm{X}=78.48$ & $(0.0,13.2,6.0)$ \\
\hline $2 \mathrm{~mm}$ teeth & $\mathrm{Y}=\left(4.47 \times 10^{7}\right) \mathrm{X}$ & $\mathrm{X}=78.30$ & $(0.0,13.3,6.0)$ \\
\hline Side Shear & & & \\
\hline $1 \mathrm{~mm}$ teeth & $\mathrm{Y}=\left(4.71 \times 10^{7}\right) \mathrm{X}$ & $\mathrm{X}=73.84$ & $(0.0,12.5,6.0)$ \\
\hline $0.5 \mathrm{~mm}$ teeth & $\mathrm{Y}=\left(4.34 \times 10^{7}\right) \mathrm{X}$ & $\mathrm{X}=80.65$ & $(0.0,13.2,6.0)$ \\
\hline $2 \mathrm{~mm}$ teeth & $\mathrm{Y}=\left(4.36 \times 10^{7}\right) \mathrm{X}$ & $\mathrm{X}=80.28$ & $(0.0,13.3,6.0)$ \\
\hline
\end{tabular}

Table 2.5: Side compression and shear point force simulation results for the three teeth designs.

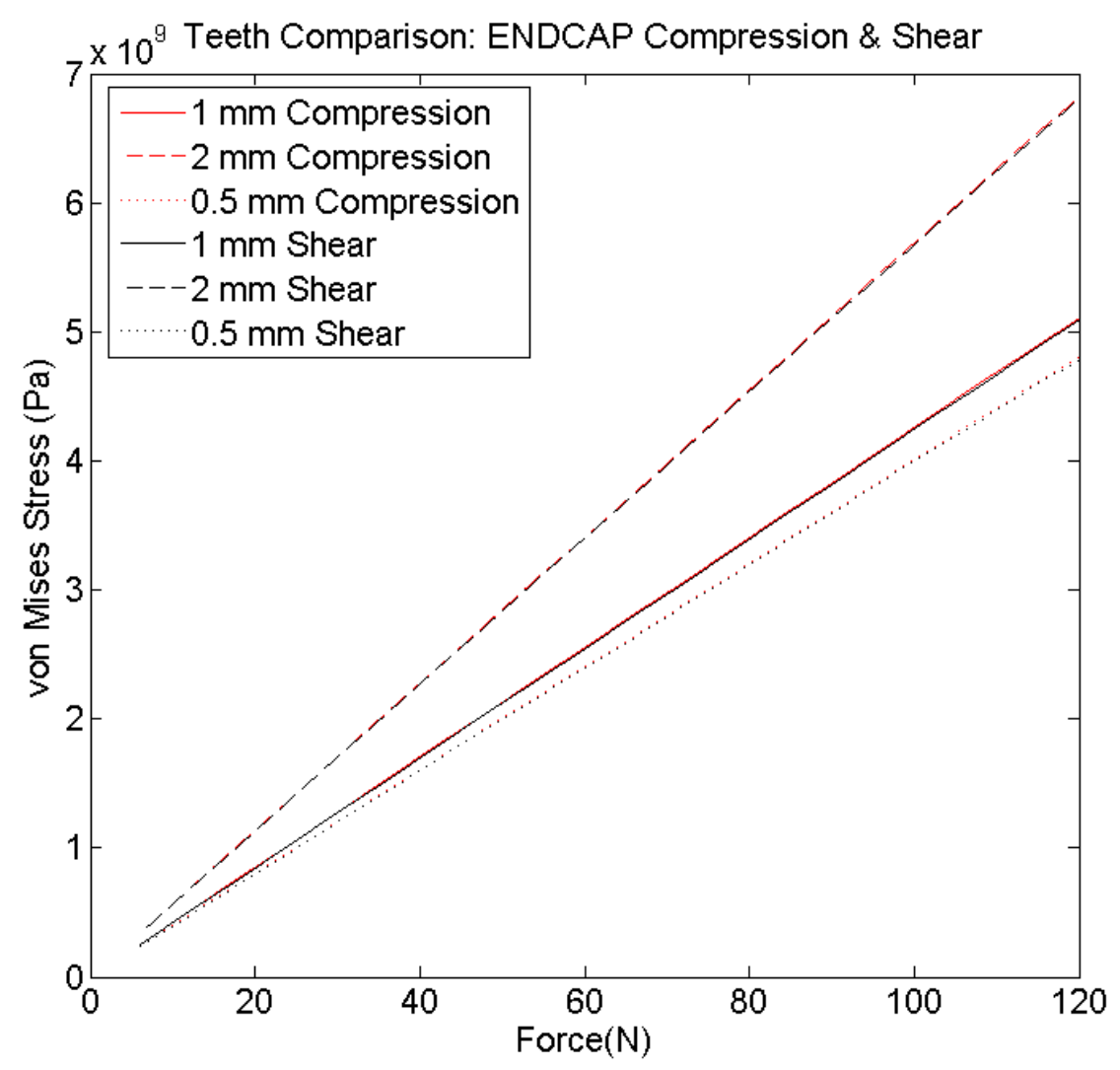

Figure 2.20: End cap compression and shear point force simulation results for the three teeth designs. 


\begin{tabular}{|r|c|c|c|}
\hline $\begin{array}{l}\text { End cap } \\
\text { Compression }\end{array}$ & $\begin{array}{c}\text { Best-Fit Linear } \\
\text { Equation }(\mathrm{Pa})\end{array}$ & $\begin{array}{c}\text { Max. Input } \\
\text { Force }(\mathrm{N})\end{array}$ & $\begin{array}{c}\text { Failure location } \\
(\mathrm{x}, \mathrm{y}, \mathrm{z}) \mathrm{mm}\end{array}$ \\
\hline $1 \mathrm{~mm}$ teeth & $\mathrm{Y}=\left(4.26 \times 10^{7}\right) \mathrm{X}$ & $\mathrm{X}=82.16$ & $(6.0,25.5,6.0)$ \\
\hline $0.5 \mathrm{~mm}$ teeth & $\mathrm{Y}=\left(4.01 \times 10^{7}\right) \mathrm{X}$ & $\mathrm{X}=87.28$ & $(6.0,25.5,6.0)$ \\
\hline $2 \mathrm{~mm}$ teeth & $\mathrm{Y}=\left(5.69 \times 10^{7}\right) \mathrm{X}$ & $\mathrm{X}=61.51$ & $(6.0,25.5,6.0)$ \\
\hline End cap Shear & & & \\
\hline $1 \mathrm{~mm}$ teeth & $\mathrm{Y}=\left(4.24 \times 10^{7}\right) \mathrm{X}$ & $\mathrm{X}=82.55$ & $(6.0,25.5,6.0)$ \\
\hline $0.5 \mathrm{~mm}$ teeth & $\mathrm{Y}=\left(3.99 \times 10^{7}\right) \mathrm{X}$ & $\mathrm{X}=87.72$ & $(6.0,25.5,6.0)$ \\
\hline $2 \mathrm{~mm}$ teeth & $\mathrm{Y}=\left(5.67 \times 10^{7}\right) \mathrm{X}$ & $\mathrm{X}=61.73$ & $(6.0,25.5,6.0)$ \\
\hline
\end{tabular}

Table 2.6: Side compression and shear point force simulation results for the three teeth designs.

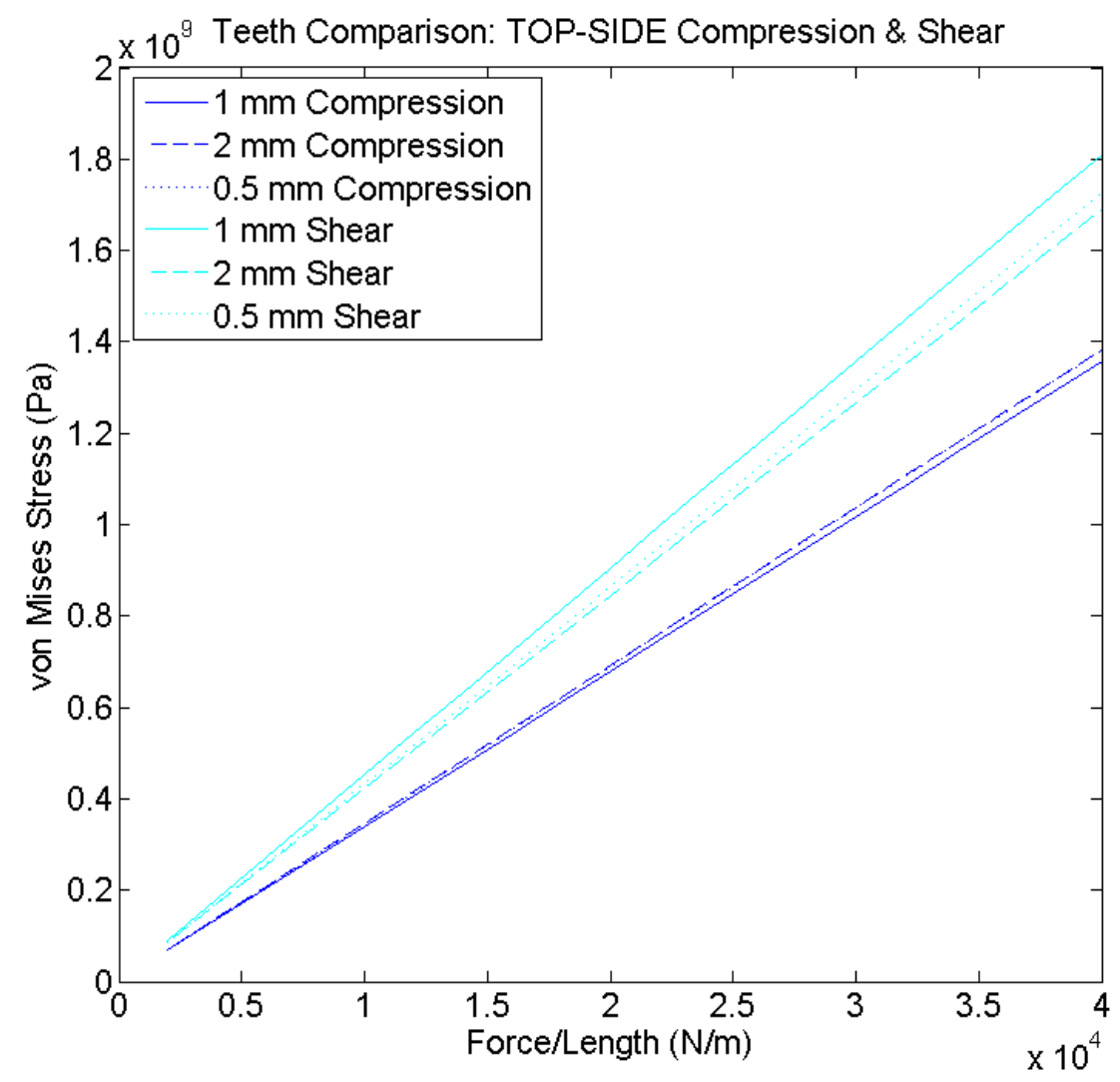

Figure 2.21: Top-Side compression and shear edge force simulation results for the three teeth designs. 


\begin{tabular}{|r|c|c|c|}
\hline $\begin{array}{l}\text { Top-Side } \\
\text { Compression }\end{array}$ & $\begin{array}{c}\text { Best-Fit Linear } \\
\text { Equation }(\mathrm{Pa})\end{array}$ & $\begin{array}{c}\text { Max. Input } \\
\text { Force/Length }(\mathrm{N} / \mathrm{m})\end{array}$ & $\begin{array}{c}\text { Failure location } \\
(\mathrm{x}, \mathrm{y}, \mathrm{z}) \mathrm{mm}\end{array}$ \\
\hline $1 \mathrm{~mm}$ teeth & $\mathrm{Y}=\left(3.39 \times 10^{4}\right) \mathrm{X}$ & $\mathrm{X}=103,245$ & $(8.0,25.5,3.2)$ \\
\hline $0.5 \mathrm{~mm}$ teeth & $\mathrm{Y}=\left(3.46 \times 10^{4}\right) \mathrm{X}$ & $\mathrm{X}=101,156$ & $(0.0,0.0,12.0)$ \\
\hline $2 \mathrm{~mm}$ teeth & $\mathrm{Y}=\left(3.46 \times 10^{4}\right) \mathrm{X}$ & $\mathrm{X}=101,156$ & $(4.0,25.3,3.2)$ \\
\hline Top-Side Shear & & & \\
\hline $1 \mathrm{~mm}$ teeth & $\mathrm{Y}=\left(4.53 \times 10^{4}\right) \mathrm{X}$ & $\mathrm{X}=77,263$ & $(8.0,25.5,3.2)$ \\
\hline $0.5 \mathrm{~mm}$ teeth & $\mathrm{Y}=\left(4.32 \times 10^{4}\right) \mathrm{X}$ & $\mathrm{X}=81,019$ & $(8.0,25.5,3.3)$ \\
\hline $2 \mathrm{~mm}$ teeth & $\mathrm{Y}=\left(4.23 \times 10^{4}\right) \mathrm{X}$ & $\mathrm{X}=82,742$ & $(8.0,25.5,3.2)$ \\
\hline
\end{tabular}

Table 2.7: Top-Side compression and shear edge force simulation results for the three teeth designs.

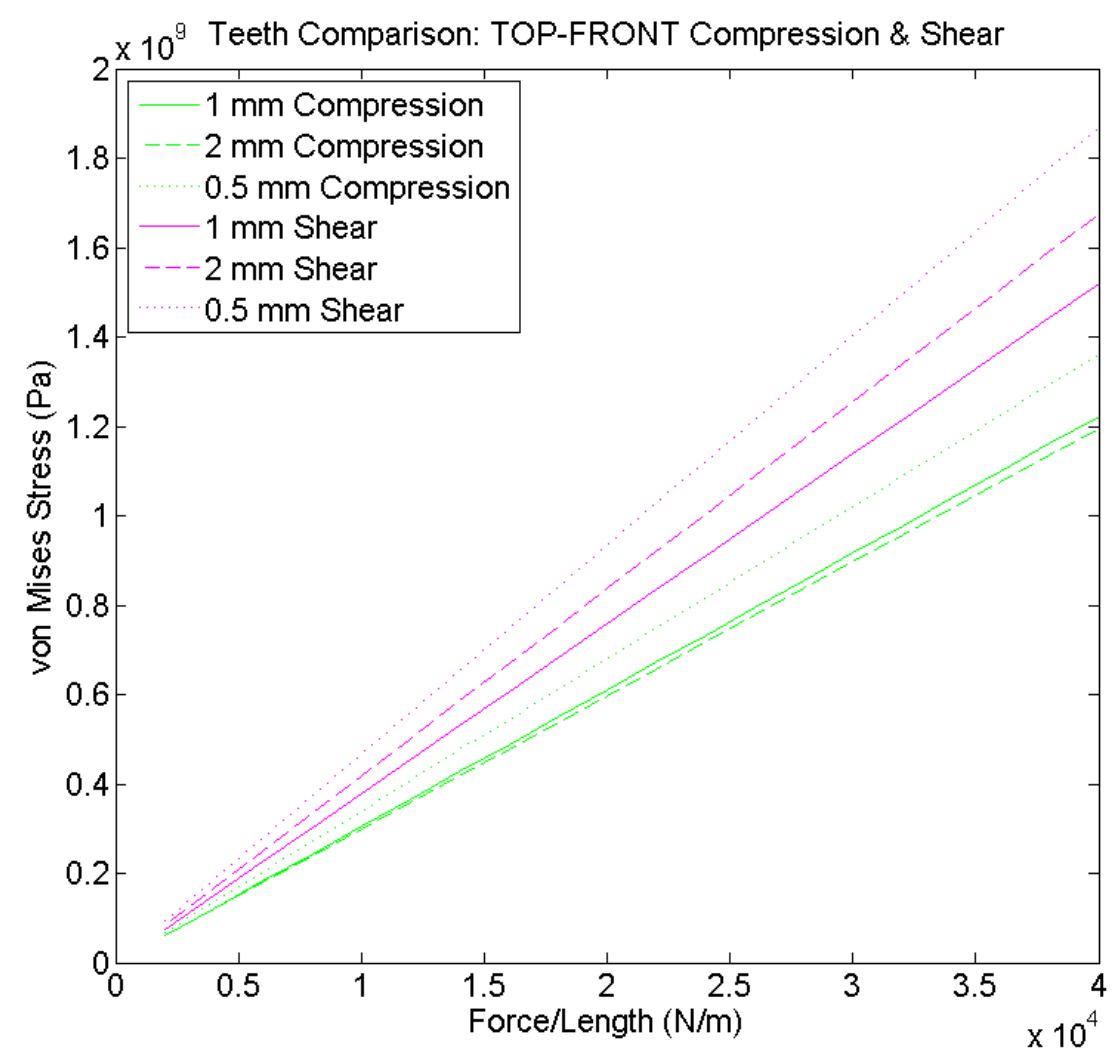

Figure 2.22: Top-Front compression and shear edge force simulation results for the three teeth designs. 


\begin{tabular}{|r|c|c|c|}
\hline $\begin{array}{l}\text { Top-Front } \\
\text { Compression }\end{array}$ & $\begin{array}{c}\text { Best-Fit Linear } \\
\text { Equation }(\mathrm{Pa})\end{array}$ & $\begin{array}{c}\text { Max. Input } \\
\text { Force/Length }(\mathrm{N} / \mathrm{m})\end{array}$ & $\begin{array}{c}\text { Failure location } \\
(\mathrm{x}, \mathrm{y}, \mathrm{z}) \mathrm{mm}\end{array}$ \\
\hline $1 \mathrm{~mm}$ teeth & $\mathrm{Y}=\left(3.05 \times 10^{4}\right) \mathrm{X}$ & $\mathrm{X}=114,754$ & $(6.2,25.5,12.0)$ \\
\hline $0.5 \mathrm{~mm}$ teeth & $\mathrm{Y}=\left(3.40 \times 10^{4}\right) \mathrm{X}$ & $\mathrm{X}=102,941$ & $(0.0,25.5,12.0)$ \\
\hline $2 \mathrm{~mm}$ teeth & $\mathrm{Y}=\left(2.99 \times 10^{4}\right) \mathrm{X}$ & $\mathrm{X}=117,057$ & $(7.0,25.5,12.0)$ \\
\hline Top-Front Shear & & & \\
\hline $1 \mathrm{~mm}$ teeth & $\mathrm{Y}=\left(3.80 \times 10^{4}\right) \mathrm{X}$ & $\mathrm{X}=92,105$ & $(12.0,25.5,12.0)$ \\
\hline $0.5 \mathrm{~mm}$ teeth & $\mathrm{Y}=\left(4.67 \times 10^{4}\right) \mathrm{X}$ & $\mathrm{X}=74,946$ & $(12.0,25.5,12.0)$ \\
\hline $2 \mathrm{~mm}$ teeth & $\mathrm{Y}=\left(4.19 \times 10^{4}\right) \mathrm{X}$ & $\mathrm{X}=83,532$ & $(12.0,25.5,12.0)$ \\
\hline
\end{tabular}

Table 2.8: Top-Front compression and shear edge force simulation results for the three teeth designs.

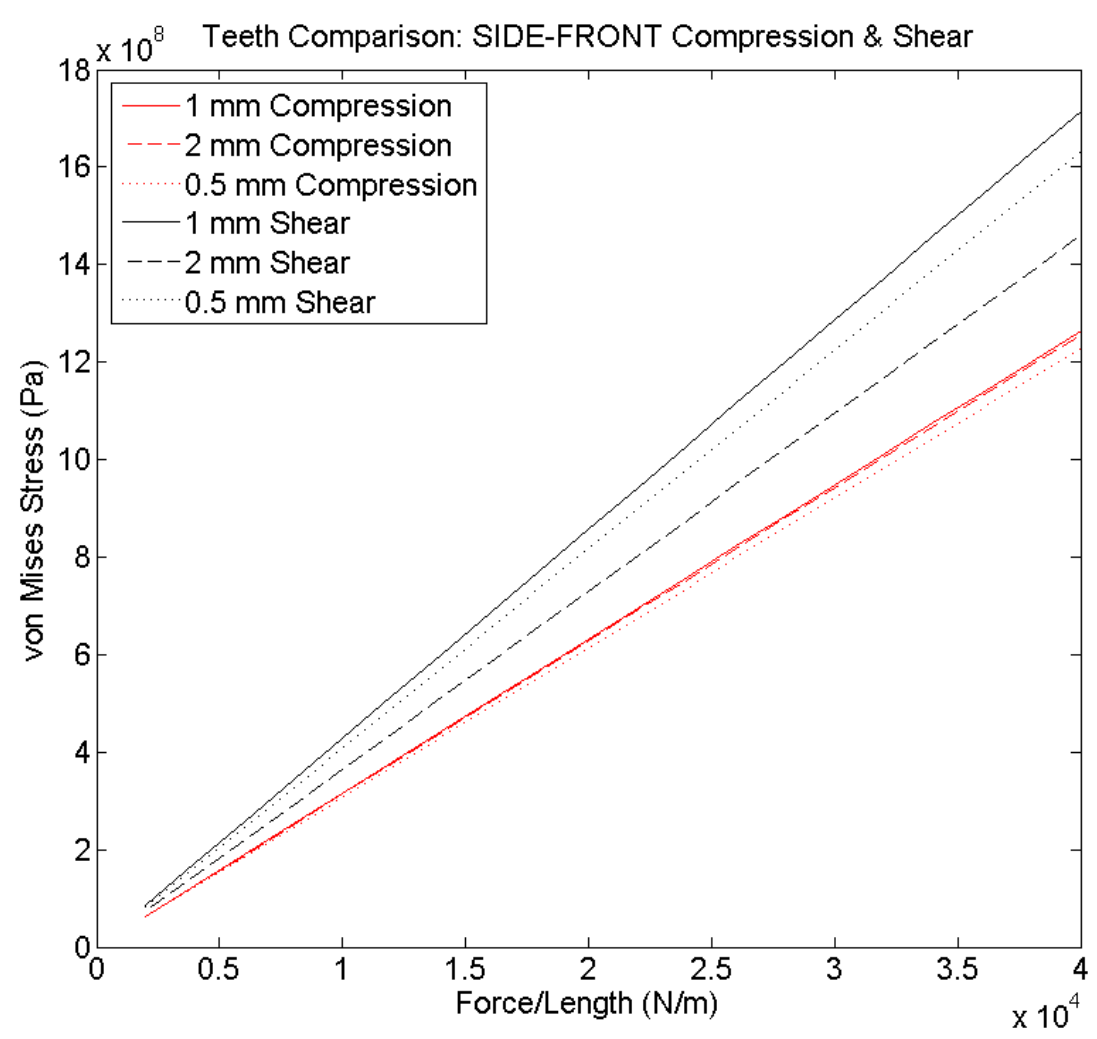

Figure 2.23: Side-Front compression and shear edge force simulation results for the three teeth designs. 


\begin{tabular}{|r|c|c|c|}
\hline $\begin{array}{l}\text { Side-Front } \\
\text { Compression }\end{array}$ & $\begin{array}{c}\text { Best-Fit Linear } \\
\text { Equation }(\mathrm{Pa})\end{array}$ & $\begin{array}{c}\text { Max. Input } \\
\text { Force/Length }(\mathrm{N} / \mathrm{m})\end{array}$ & $\begin{array}{c}\text { Failure location } \\
(\mathrm{x}, \mathrm{y}, \mathrm{z}) \mathrm{mm}\end{array}$ \\
\hline $1 \mathrm{~mm}$ teeth & $\mathrm{Y}=\left(3.16 \times 10^{4}\right) \mathrm{X}$ & $\mathrm{X}=110,759$ & $(0.0,25.5,10.5)$ \\
\hline $0.5 \mathrm{~mm}$ teeth & $\mathrm{Y}=\left(3.07 \times 10^{4}\right) \mathrm{X}$ & $\mathrm{X}=114,007$ & $(0.0,25.5,9.3)$ \\
\hline $2 \mathrm{~mm}$ teeth & $\mathrm{Y}=\left(3.14 \times 10^{4}\right) \mathrm{X}$ & $\mathrm{X}=111,465$ & $(0.0,25.5,9.0)$ \\
\hline Side-Front Shear & & & \\
\hline $1 \mathrm{~mm}$ teeth & $\mathrm{Y}=\left(4.29 \times 10^{4}\right) \mathrm{X}$ & $\mathrm{X}=81,585$ & $(0.0,25.5,0.0)$ \\
\hline $0.5 \mathrm{~mm}$ teeth & $\mathrm{Y}=\left(4.08 \times 10^{4}\right) \mathrm{X}$ & $\mathrm{X}=85,784$ & $(0.0,25.5,0.0)$ \\
\hline $2 \mathrm{~mm}$ teeth & $\mathrm{Y}=\left(3.65 \times 10^{4}\right) \mathrm{X}$ & $\mathrm{X}=95,890$ & $(0.0,25.5,0.0)$ \\
\hline
\end{tabular}

Table 2.9: Side-Front compression and shear edge force simulation results for the three teeth designs.

Some key observations regarding the three teeth design simulation results are that the $2 \mathrm{~mm}$ long teeth features are generally stronger for pressure and edge forces exerted on the platform. One exception is for the end cap compression pressure case where the $0.5 \mathrm{~mm}$ teeth design is stronger by approximately $420 \mathrm{kPa}$. Also, in the end cap compression point force case the $0.5 \mathrm{~mm}$ teeth design is roughly $25 \mathrm{~N}$ stronger than the other designs.

\subsection{Platform Structural Mechanics FEM Analysis}

The following section compares the mechanical strength of two $12 \times 12 \times 25 \mathrm{~mm}^{3}$ platform types: (i) one that implements the Hook-Hole features described earlier in this chapter; and (ii) one that implements the $1 \mathrm{~mm}$ interdigitated teeth design. The analysis is performed using COMSOL MultiPhysics ${ }^{\mathrm{TM}}$ finite-element modeling software with the following silicon material properties: Young's Modulus =186.5 GPa; Poisson's Ratio = 0.26 ; and the density $=2,329 \mathrm{~kg} / \mathrm{m}^{3}$. These properties are used to model a (111) oriented silicon wafer. 


\subsubsection{Pressure and Point Force Simulation Results}

For the pressure simulations, the pressure was applied in the normal direction, evenly across the platform face and varied from $0.25 \mathrm{MPa}$ to 5.0 $\mathrm{MPa}$. The point force simulations were also applied in the normal direction at a point directly in the center of the platform face and varied from $6 \mathrm{~N}$ to $120 \mathrm{~N}$. Figures 2.24 and 2.25 show the results for the pressure simulations, and Figs. 2.26 and 2.27 are the results for the point force simulations.

From Figs. 2.24 to 2.25, the generated stresses are three to four times lower for the platform configuration that has the $1 \mathrm{~mm}$ interdigitated teeth features. Therefore, the platform configuration with the $1 \mathrm{~mm}$ teeth features is more likely to be mechanically stronger. Based on the simulation results, the platform is stronger for applied pressure in compression than shear pressure. Figures 2.26 and 2.27 shows the resulting stresses are similar for both compression and shear point forces, except for Top side point forces on the Hook-Hole configuration as seen in Fig. 2.27, since the Top side has hole features around the entire periphery.

Since the interdigitated teeth platform pieces all have the same periphery features, the simulated stress variance is much lower compared to the Hook-Hole configuration which does not. Another observation is the simulated stresses are lower for the End cap faces, which have a smaller surface area compared to the Top and Side faces. The reduction in overall platform volume to increase mechanical strength will be investigated in Section 2.4 of this chapter. 


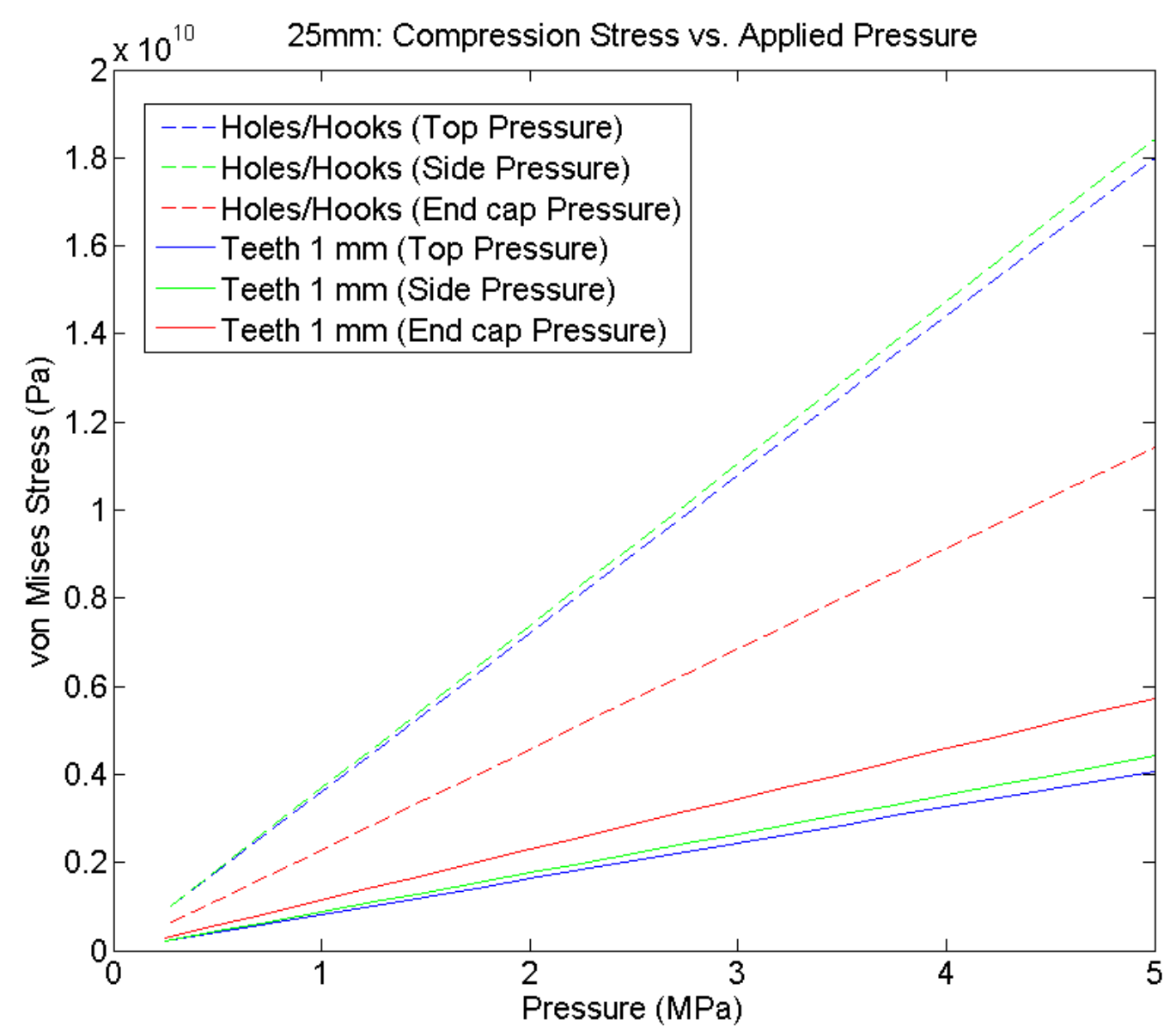

Figure 2.24: Pressure, compression stress simulation results.

\begin{tabular}{|r|c|c|c|}
\hline $\begin{array}{l}\text { Compression } \\
\text { Holes/Hooks }\end{array}$ & $\begin{array}{c}\text { Best-Fit Linear } \\
\text { Equation }(\mathrm{Pa})\end{array}$ & $\begin{array}{c}\text { Max. Input } \\
\text { Pressure }(\mathrm{MPa})\end{array}$ & $\begin{array}{c}\text { Failure location } \\
(\mathrm{x}, \mathrm{y}, \mathrm{z}) \mathrm{mm}\end{array}$ \\
\hline Top & $\mathrm{Y}=(3601) \mathrm{X}$ & $\mathrm{X}=0.97$ & $(11.8,5.7,12.0)$ \\
\hline Side & $\mathrm{Y}=(3684) \mathrm{X}$ & $\mathrm{X}=0.95$ & $(0.25,17.7,0.0)$ \\
\hline End cap & $\mathrm{Y}=(2285) \mathrm{X}$ & $\mathrm{X}=1.53$ & $(3.1,25.2,12.0)$ \\
\hline 1 $\mathrm{mm}$ teeth & & & \\
\hline Top & $\mathrm{Y}=(814.7) \mathrm{X}$ & $\mathrm{X}=4.30$ & $(0.125,10.1,12.0)$ \\
\hline Side & $\mathrm{Y}=(882.8) \mathrm{X}$ & $\mathrm{X}=3.96$ & $(0.125,10.1,12.0)$ \\
\hline End cap & $\mathrm{Y}=(1145.9) \mathrm{X}$ & $\mathrm{X}=3.05$ & $(8.0,25.3,0.250)$ \\
\hline
\end{tabular}

Table 2.10: Pressure, compression stress simulation results. 


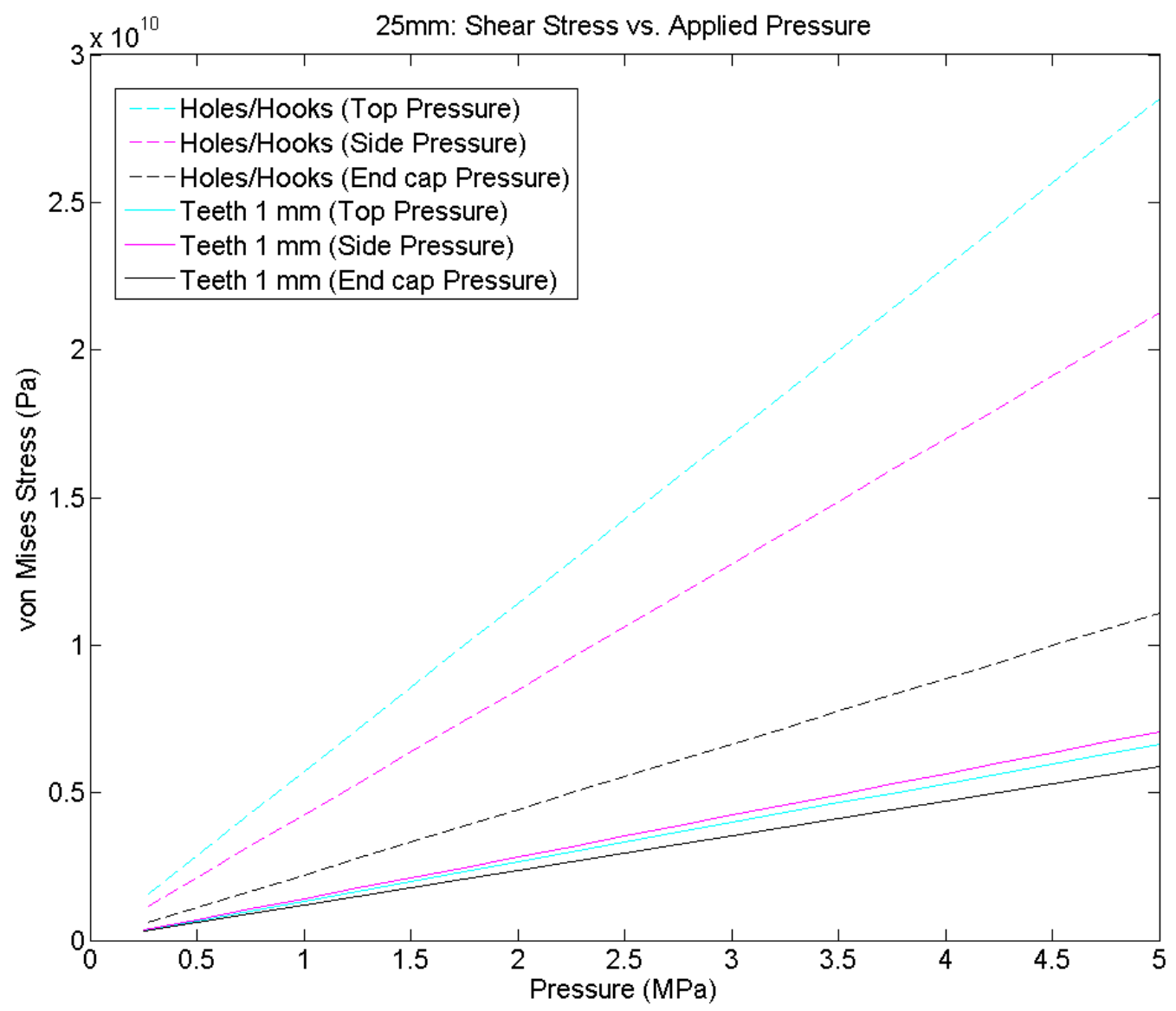

Figure 2.25: Pressure, shear stress simulation results.

\begin{tabular}{|r|c|c|c|}
\hline $\begin{array}{l}\text { Shear } \\
\text { Holes/Hooks }\end{array}$ & $\begin{array}{c}\text { Best-Fit Linear } \\
\text { Equation }(\mathrm{Pa})\end{array}$ & $\begin{array}{c}\text { Max. Input } \\
\text { Pressure }(\mathrm{MPa})\end{array}$ & $\begin{array}{c}\text { Failure location } \\
(\mathrm{x}, \mathrm{y}, \mathrm{z}) \mathrm{mm}\end{array}$ \\
\hline Top & $\mathrm{Y}=(5702.2) \mathrm{X}$ & $\mathrm{X}=0.61$ & $(11.8,11.4,12.0)$ \\
\hline Side & $\mathrm{Y}=(4249.0) \mathrm{X}$ & $\mathrm{X}=0.82$ & $(0.25,9.4,0.25$ \\
\hline End cap & $\mathrm{Y}=(2217.3) \mathrm{X}$ & $\mathrm{X}=1.58$ & $(3.1,25.2,8.1)$ \\
\hline $1 \mathrm{~mm}$ teeth & & & \\
\hline Top & $\mathrm{Y}=(1329.1) \mathrm{X}$ & $\mathrm{X}=2.63$ & $(11.8,12.9,11.7)$ \\
\hline Side & $\mathrm{Y}=(1412.5) \mathrm{X}$ & $\mathrm{X}=2.48$ & $(0.25,9.9,0.25)$ \\
\hline End cap & $\mathrm{Y}=(1180.3) \mathrm{X}$ & $\mathrm{X}=2.97$ & $(8.0,25.3,0.25)$ \\
\hline
\end{tabular}

Table 2.11: Pressure, shear stress simulation results. 


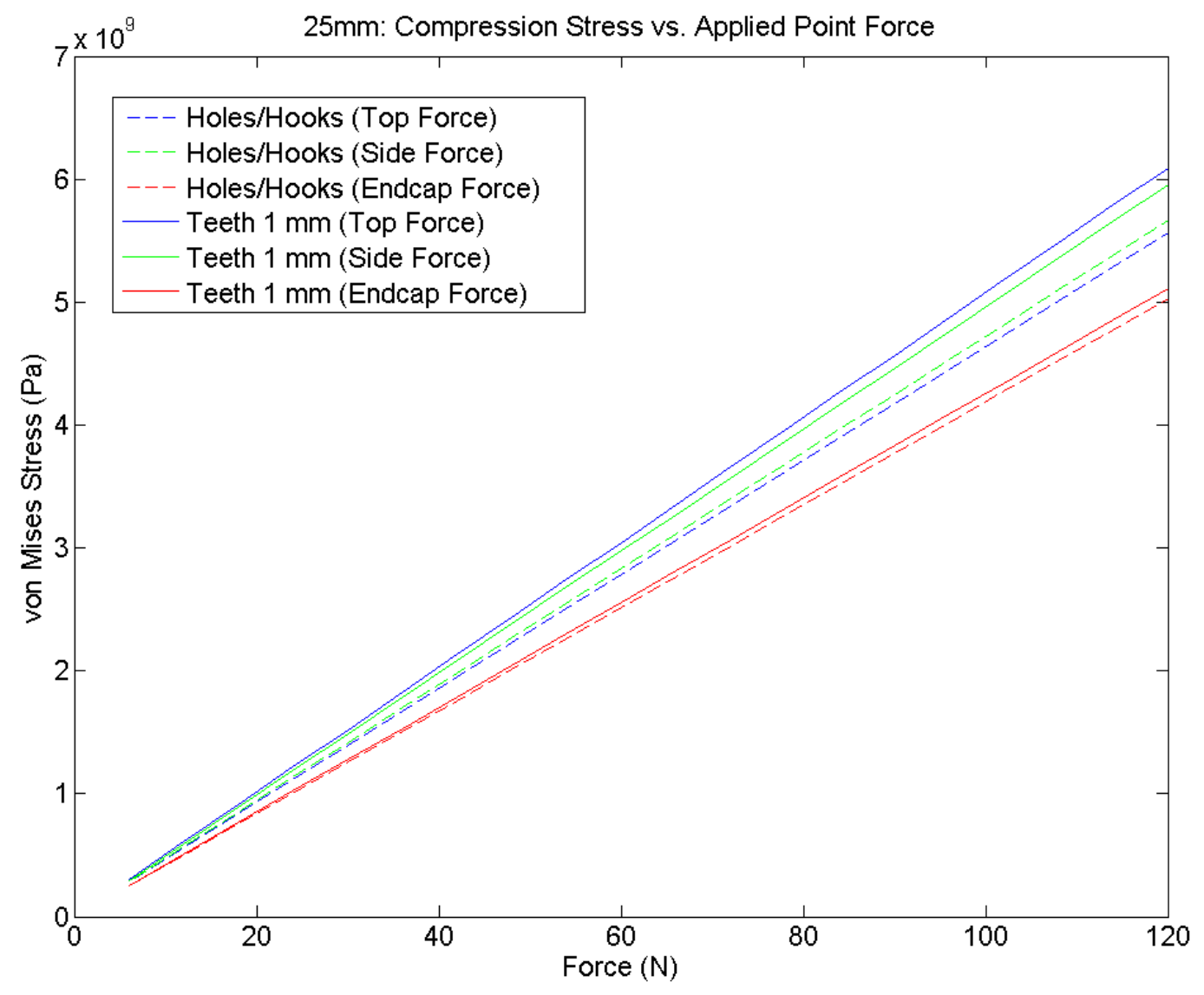

Figure 2.26: Point force, compression stress simulation results.

\begin{tabular}{|r|c|c|c|}
\hline $\begin{array}{l}\text { Compression } \\
\text { Holes/Hooks }\end{array}$ & $\begin{array}{c}\text { Best-Fit Linear } \\
\text { Equation }(\mathrm{Pa})\end{array}$ & $\begin{array}{c}\text { Max. Input } \\
\text { Force }(\mathrm{N})\end{array}$ & $\begin{array}{c}\text { Failure location } \\
(\mathrm{x}, \mathrm{y}, \mathrm{z}) \mathrm{mm}\end{array}$ \\
\hline Top & $\mathrm{Y}=\left(4.63 \times 10^{7}\right) \mathrm{X}$ & $\mathrm{X}=75.59$ & $(11.8,11.7,12.0)$ \\
\hline Side & $\mathrm{Y}=\left(4.72 \times 10^{7}\right) \mathrm{X}$ & $\mathrm{X}=74.15$ & $(0.25,14.0,0.05)$ \\
\hline End cap & $\mathrm{Y}=\left(4.18 \times 10^{7}\right) \mathrm{X}$ & $\mathrm{X}=83.73$ & $(0.25,25.4,8.1)$ \\
\hline $1 \mathrm{~mm}$ teeth & & & \\
\hline Top & $\mathrm{Y}=\left(5.08 \times 10^{7}\right) \mathrm{X}$ & $\mathrm{X}=68.90$ & $(6.0,12.5,12.0)$ \\
\hline Side & $\mathrm{Y}=\left(4.71 \times 10^{7}\right) \mathrm{X}$ & $\mathrm{X}=74.31$ & $(0.0,12.5,6.0)$ \\
\hline End cap & $\mathrm{Y}=\left(4.24 \times 10^{7}\right) \mathrm{X}$ & $\mathrm{X}=82.55$ & $(6.0,25.5,6.0)$ \\
\hline
\end{tabular}

Table 2.12: Point force, compression stress simulation results. 


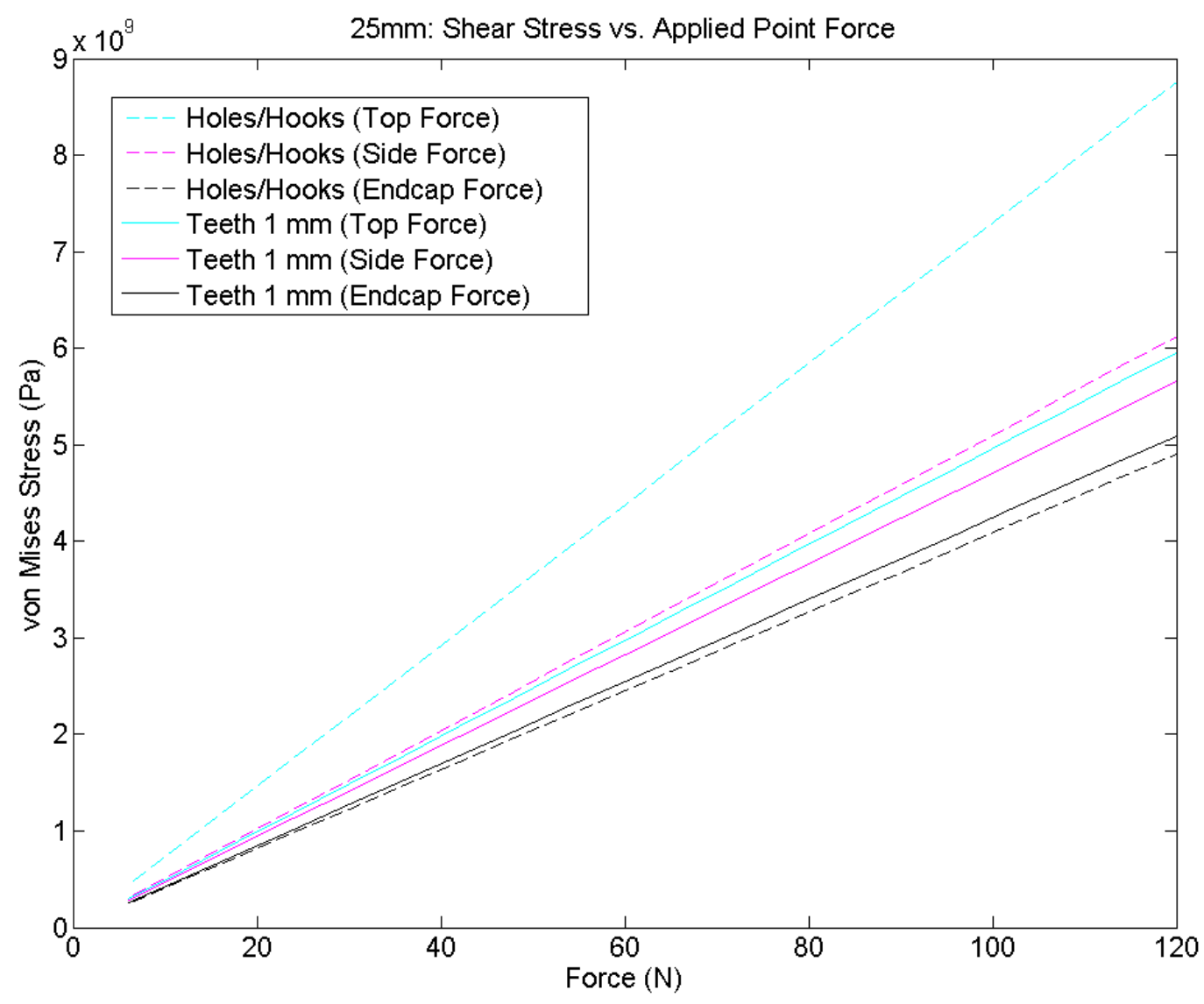

Figure 2.27: Point force, shear stress simulation results.

\begin{tabular}{|r|c|c|c|}
\hline $\begin{array}{l}\text { Shear } \\
\text { Holes/Hooks }\end{array}$ & $\begin{array}{c}\text { Best-Fit Linear } \\
\text { Equation }(\mathrm{Pa})\end{array}$ & $\begin{array}{c}\text { Max. Input } \\
\text { Force }(\mathrm{N})\end{array}$ & $\begin{array}{c}\text { Failure location } \\
(\mathrm{x}, \mathrm{y}, \mathrm{z}) \mathrm{mm}\end{array}$ \\
\hline Top & $\mathrm{Y}=\left(7.30 \times 10^{7}\right) \mathrm{X}$ & $\mathrm{X}=47.94$ & $(11.8,11.4,12.0)$ \\
\hline Side & $\mathrm{Y}=\left(5.10 \times 10^{7}\right) \mathrm{X}$ & $\mathrm{X}=68.63$ & $(0.25,12.1,0.25)$ \\
\hline End cap & $\mathrm{Y}=\left(4.10 \times 10^{7}\right) \mathrm{X}$ & $\mathrm{X}=85.37$ & $(0.25,25.4,8.1)$ \\
\hline $1 \mathrm{~mm}$ teeth & & & \\
\hline Top & $\mathrm{Y}=\left(4.96 \times 10^{7}\right) \mathrm{X}$ & $\mathrm{X}=70.56$ & $(6.0,12.5,12.0)$ \\
\hline Side & $\mathrm{Y}=\left(4.71 \times 10^{7}\right) \mathrm{X}$ & $\mathrm{X}=74.31$ & $(0.0,12.5,6.0)$ \\
\hline End cap & $\mathrm{Y}=\left(4.24 \times 10^{7}\right) \mathrm{X}$ & $\mathrm{X}=82.55$ & $(6.0,25.5,6.0)$ \\
\hline
\end{tabular}

Table 2.13: Point force, shear stress simulation results. 


\subsubsection{Edge Force Finite Element Simulation Results}

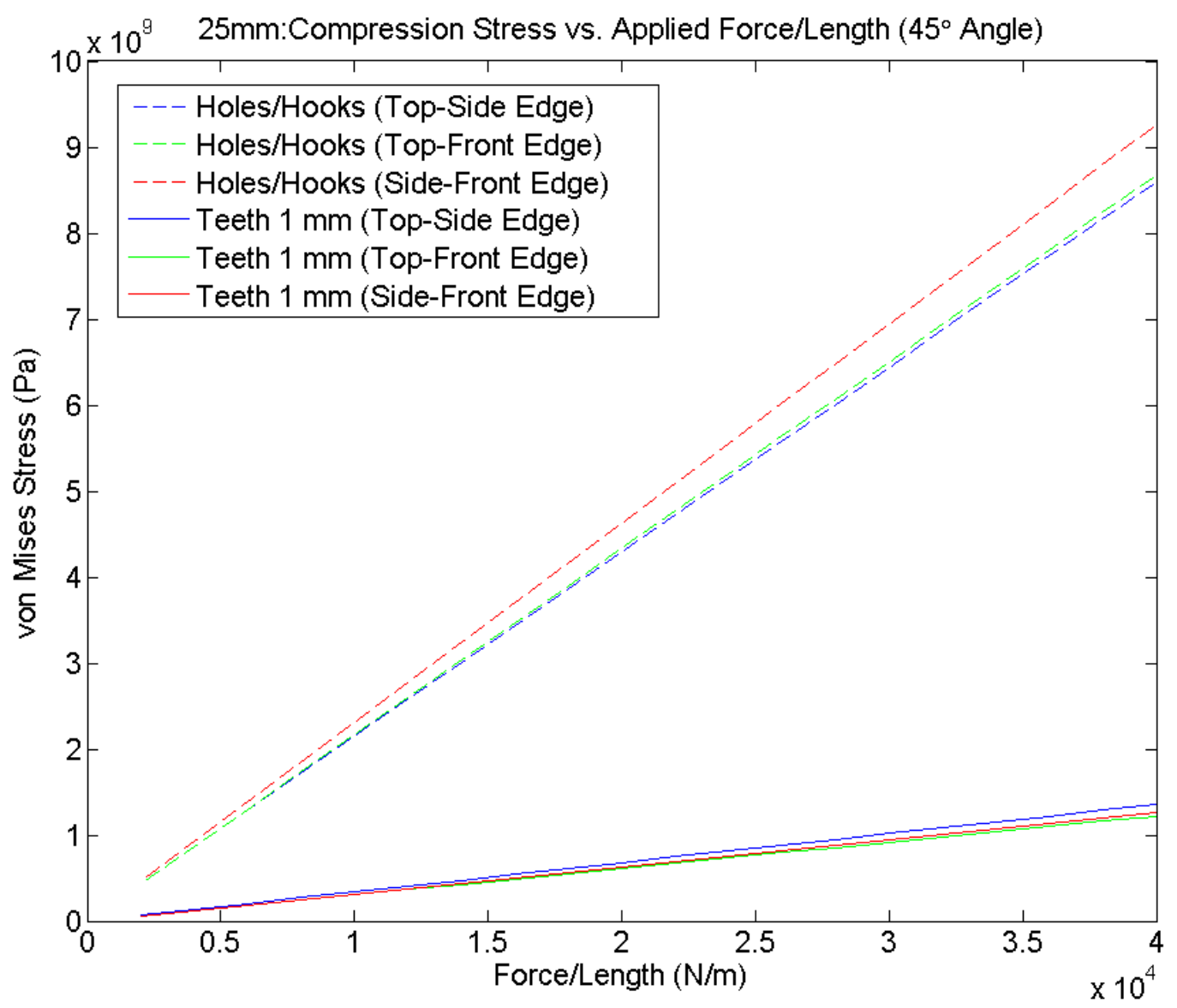

Figure 2.28: Edge force, compression stress simulation results.

\begin{tabular}{|r|c|c|c|}
\hline $\begin{array}{l}\text { Compression } \\
\text { Holes/Hooks }\end{array}$ & $\begin{array}{c}\text { Best-Fit Linear } \\
\text { Equation }(\mathrm{Pa})\end{array}$ & $\begin{array}{c}\text { Max. Input } \\
\text { Force/Length }(\mathrm{N} / \mathrm{m})\end{array}$ & $\begin{array}{c}\text { Failure location } \\
(\mathrm{x}, \mathrm{y}, \mathrm{z}) \mathrm{mm}\end{array}$ \\
\hline Top-Side & $\mathrm{Y}=\left(1.78 \times 10^{5}\right) \mathrm{X}$ & $\mathrm{X}=19,662.9$ & $(0.0,12.0,12.0)$ \\
\hline Top-Front & $\mathrm{Y}=\left(1.81 \times 10^{5}\right) \mathrm{X}$ & $\mathrm{X}=19,337.0$ & $(1.2,25.4,12.0)$ \\
\hline Side-Front & $\mathrm{Y}=\left(2.89 \times 10^{5}\right) \mathrm{X}$ & $\mathrm{X}=12,110.7$ & $(0.0,25.5,10.3)$ \\
\hline $1 \mathrm{~mm}$ teeth & & & \\
\hline Top-Side & $\mathrm{Y}=\left(3.39 \times 10^{4}\right) \mathrm{X}$ & $\mathrm{X}=103,244.8$ & $(8.0,25.5,3.2)$ \\
\hline Top-Front & $\mathrm{Y}=\left(3.05 \times 10^{4}\right) \mathrm{X}$ & $\mathrm{X}=114,754.1$ & $(6.2,25.5,12.0)$ \\
\hline Front-Side & $\mathrm{Y}=\left(3.16 \times 10^{4}\right) \mathrm{X}$ & $\mathrm{X}=110,759.5$ & $(0.0,25.5,10.5)$ \\
\hline
\end{tabular}

Table 2.14: Edge force, compression stress simulation results. 


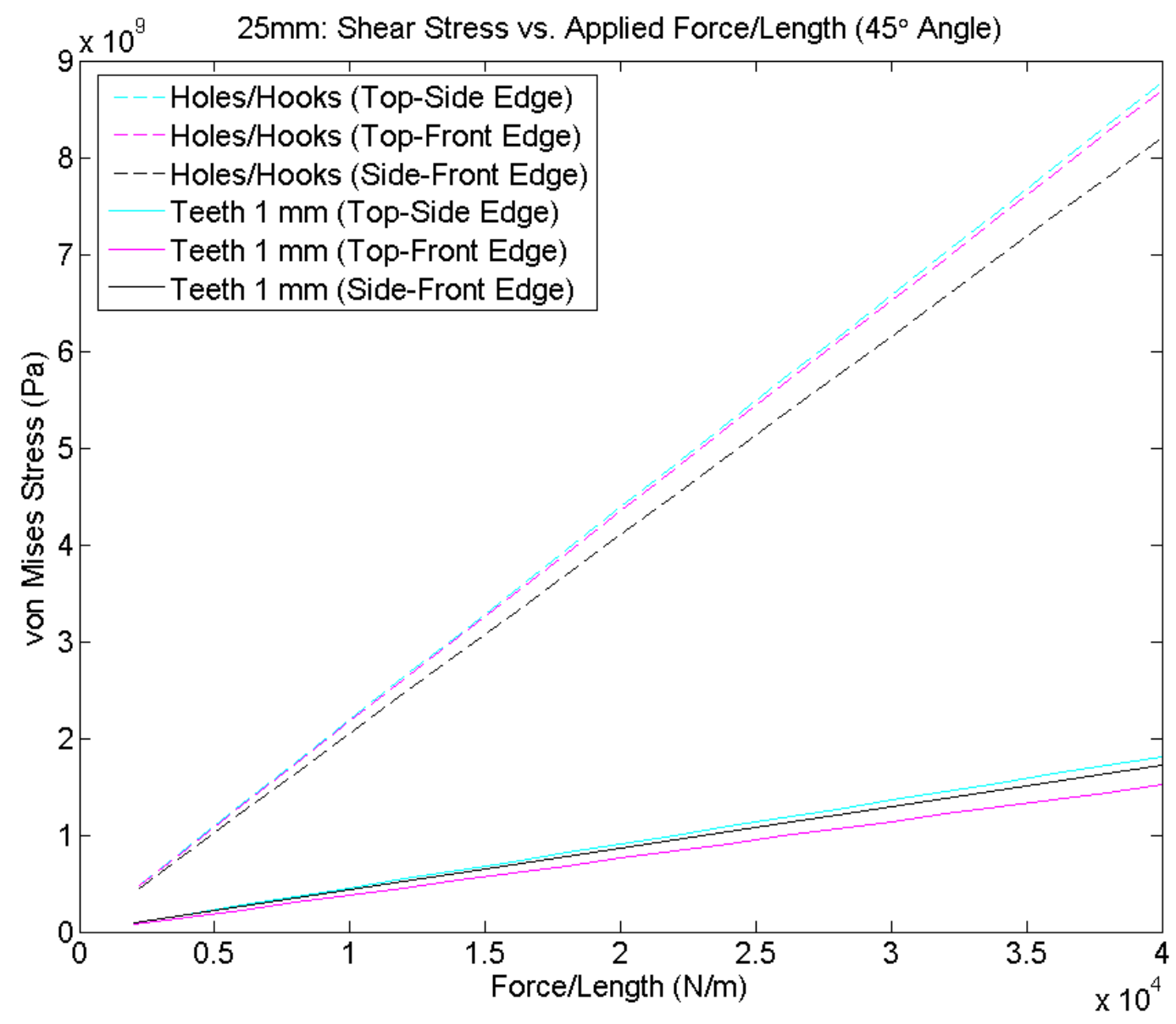

Figure 2.29: Edge force, shear stress simulation results.

\begin{tabular}{|r|c|c|c|}
\hline $\begin{array}{l}\text { Shear } \\
\text { Holes/Hooks }\end{array}$ & $\begin{array}{c}\text { Best-Fit Linear } \\
\text { Equation }(\mathrm{Pa})\end{array}$ & $\begin{array}{c}\text { Max. Input } \\
\text { Force/Length }(\mathrm{N} / \mathrm{m})\end{array}$ & $\begin{array}{c}\text { Failure location } \\
(\mathrm{x}, \mathrm{y}, \mathrm{z}) \mathrm{mm}\end{array}$ \\
\hline Top-Side & $\mathrm{Y}=\left(2.88 \times 10^{5}\right) \mathrm{X}$ & $\mathrm{X}=12,152.8$ & $(0.0,12.0,12.0)$ \\
\hline Top-Front & $\mathrm{Y}=\left(1.81 \times 10^{5}\right) \mathrm{X}$ & $\mathrm{X}=19,337.0$ & $(1.2,25.4,12.0)$ \\
\hline Front-Side & $\mathrm{Y}=\left(1.78 \times 10^{5}\right) \mathrm{X}$ & $\mathrm{X}=19,662.9$ & $(0.0,25.5,10.5)$ \\
\hline $1 \mathrm{~mm}$ teeth & & & \\
\hline Top-Side & $\mathrm{Y}=\left(4.53 \times 10^{4}\right) \mathrm{X}$ & $\mathrm{X}=77,262.7$ & $(8.0,25.3,3.2)$ \\
\hline Top-Front & $\mathrm{Y}=\left(3.80 \times 10^{4}\right) \mathrm{X}$ & $\mathrm{X}=92,105.3$ & $(12.0,25.5,12.0)$ \\
\hline Front-Side & $\mathrm{Y}=\left(4.29 \times 10^{4}\right) \mathrm{X}$ & $\mathrm{X}=81,585.1$ & $(0.0,25.5,0.0)$ \\
\hline
\end{tabular}

Table 2.15: Edge force, shear stress simulation results. 
From Figs. 2.28 and 2.29, it is obvious that eliminating the Hook-Hole features from the platform greatly reduces the generated stresses and makes the structure more mechanically sound during edge applied forces.

In summary, replacing the Hook-Hole features with simple rectangular, interdigitated teeth features increases the overall mechanical strength of the platform by three to five times depending on the type of applied force. Furthermore, since all the platform pieces have identical periphery features, the variance in the generated stresses is reduced for applied area, point and edge forces.

\subsubsection{Analytical Verification of Finite Element Simulation Results}

To understand how close the platform finite-element modeling simulations compare to analytically derived results, two applied pressure scenarios will be analyzed, including Side Compression and Side Shear. Using the Matlab ${ }^{\circledR}$ function, polyfit(), the best-fit linear equations for the simulated results were calculated. For the analytical calculations, the following formula was used to calculate the resulting maximum stress from a uniform area force applied to a fixed plate [70]:

$$
\sigma_{\max }=\frac{\beta q b^{2}}{t^{2}} \quad \text { Analytic equation for stress calculation }
$$

where $\mathrm{q}$ is the applied area force, $\mathrm{b}$ is the length of the plate's shortest side $(12 \mathrm{~mm})$ and $\mathrm{t}$ is the plate thickness $(0.250 \mathrm{~mm})$. The term $\beta,(\mathrm{a} / \mathrm{b})$, is a constant determined from the analytical tables where, a, is the length of the plate's longest side $(25 \mathrm{~mm})$. The values found for $\beta$ are 0.4974 and 0.73 for the Side compression and Side shear cases, 
respectively. The input force to output stress equations for the finite-element simulation results and from evaluating (Eq. (2.1)) are presented in Table 2.16.

\begin{tabular}{|r|c|c|c|}
\hline $\begin{array}{c}\text { Applied Area Force } \\
\text { Scenario }\end{array}$ & $\begin{array}{c}\text { Simulation Result } \\
(\max )\end{array}$ & $\begin{array}{c}\text { Analytical Result } \\
(\max )\end{array}$ & \% Difference \\
\hline Side Compression & $\sigma=(882.613) \times q$ & $\sigma=(1146.001) \times q$ & 29.98 \\
\hline Side Shear & $\sigma=(1412.629) \times q$ & $\sigma=(1681.920) \times q$ & 19.06 \\
\hline
\end{tabular}

Table 2.16: Comparison between simulation and analytical calculated stress results.

From Table 2.16 above, it is shown that the analytical results produce a higher calculated stress than the simulated results. The difference between the two methods for the Side Compression scenario arises from the analytical case assuming only the edges of the plate are fixed where the simulation case has a $250 \mu \mathrm{m}$ thick peripheral segment fixing the plate. The Side Shear scenario shows less difference between the two as the analytical scenario more resembles the simulated platform structure boundary conditions. In general, the analytical results give good first order approximations to the simulated finite-element modeling results. 


\subsection{Platform Size Reduction and Mechanical Strength Analysis}

\subsubsection{Finite-Element Modeling Simulations}

Volume reduction is important to enhance insertion opportunities where space as at a premium and for potentially lowering manufacturing costs. Figure 6.1 depicts four types of platform configurations, two which have been realized and a future $1.5 \mathrm{~mm}^{3}$ and $12 \mathrm{~mm}^{3}$ cubic platforms. Platform volume reduction and maintaining a cubic shape dramatically increases the overall mechanical strength of the platform as shown in Fig. 2.30 for applied pressure simulations for the three platform types.

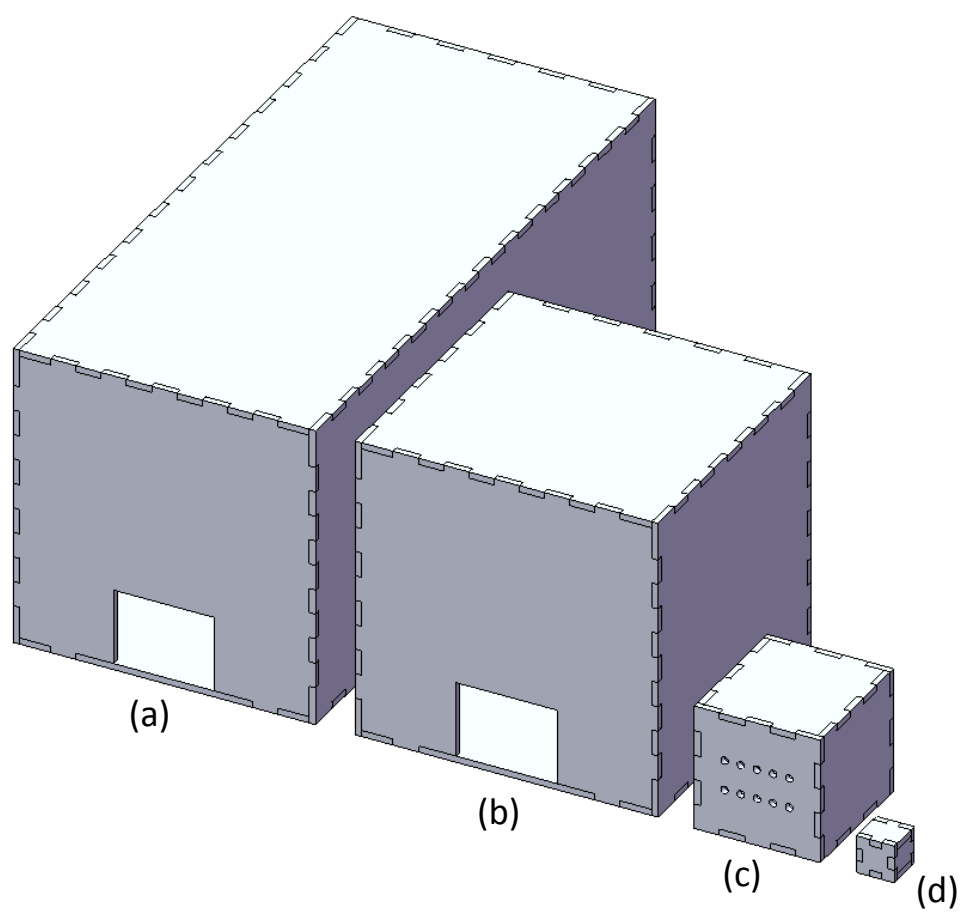

Figure 2.30: Different platform realizations: (a) $12 \times 12 \times 25 \mathrm{~mm}^{3}$; (b) $12 \mathrm{~mm}^{3}$; (c) $5 \mathrm{~mm}^{3}$ cube; and (d) $1.5 \mathrm{~mm}^{3}$ cube. All have $1 \mathrm{~mm}$ interdigitated teeth features except for the $1.5 \mathrm{~mm}^{3}$ cube which has $0.5 \mathrm{~mm}$ features. 


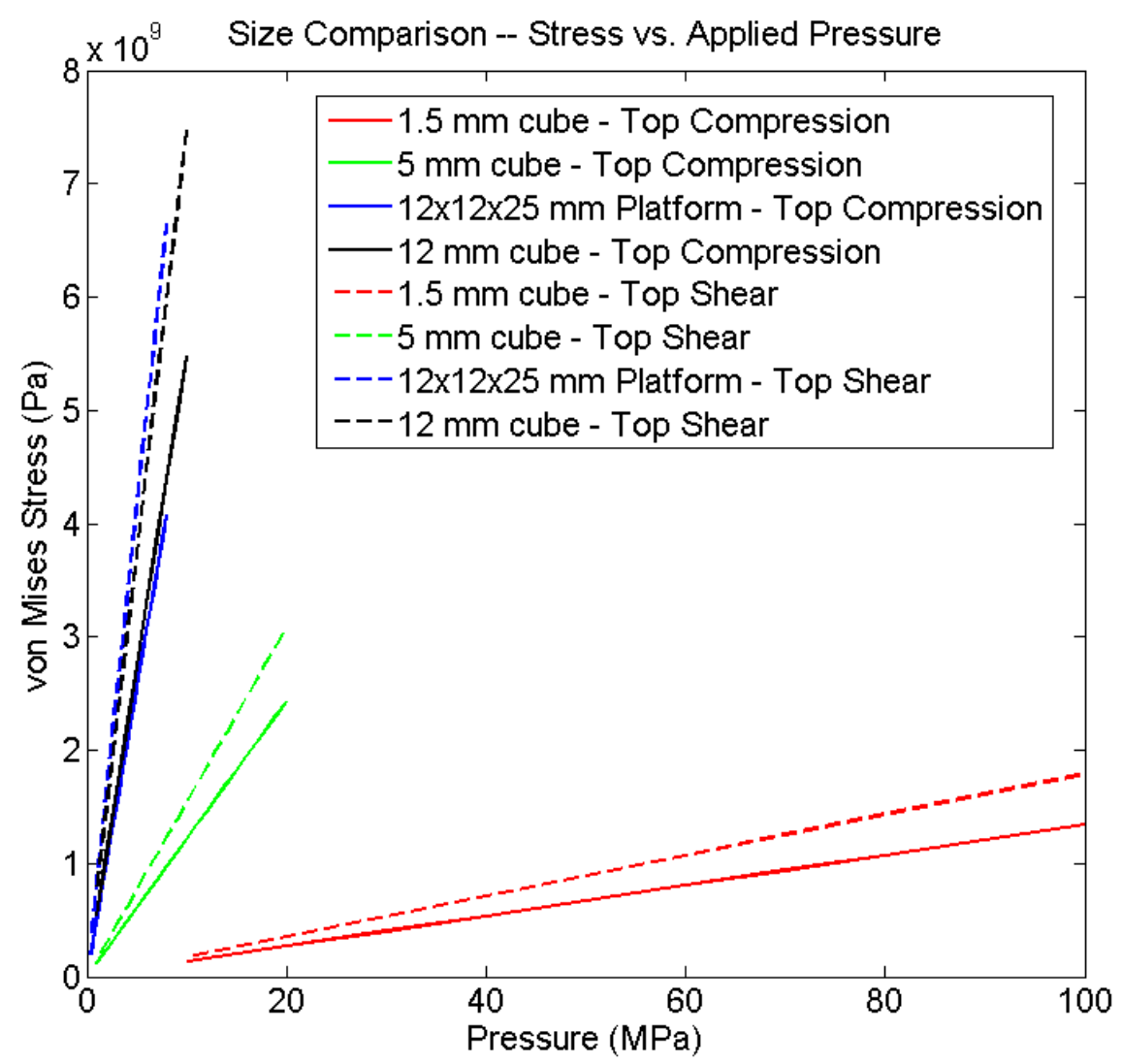

Figure 2.31: Platform size comparisons for simulated stress versus applied pressure.

\begin{tabular}{|r|c|c|c|}
\hline \multicolumn{1}{|l|}{ Compression } & $\begin{array}{c}\text { Best-Fit Linear } \\
\text { Equation }(\mathrm{Pa})\end{array}$ & $\begin{array}{c}\text { Max. Input } \\
\text { Pressure }(\mathrm{MPa})\end{array}$ & $\begin{array}{c}\text { Failure Location } \\
(\mathrm{x}, \mathrm{y}, \mathrm{z}) \mathrm{in} \mathrm{mm}\end{array}$ \\
\hline $1.5 \mathrm{~mm}$ Cube & $\mathrm{Y}=\left(1.34 \times 10^{7}\right) \mathrm{X}$ & $\mathrm{X}=261.11$ & $(0.0,0.0,1.4)$ \\
\hline $5 \mathrm{~mm}$ Cube & $\mathrm{Y}=\left(1.21 \times 10^{8}\right) \mathrm{X}$ & $\mathrm{X}=28.86$ & $(4.7,3.7,4.8)$ \\
\hline $12 \times 12 \times 25 \mathrm{~mm}$ & $\mathrm{Y}=\left(8.15 \times 10^{8}\right) \mathrm{X}$ & $\mathrm{X}=4.30$ & $(0.125,10.1,12.0)$ \\
\hline $12 \mathrm{~mm}$ Cube & $\mathrm{Y}=\left(5.48 \times 10^{8}\right) \mathrm{X}$ & $\mathrm{X}=6.39$ & $(5.1,12.0,11.9)$ \\
\hline Shear & & & \\
\hline $1.5 \mathrm{~mm}$ Cube & $\mathrm{Y}=\left(1.79 \times 10^{7}\right) \mathrm{X}$ & $\mathrm{X}=195.82$ & $(1.2,0.0,1.4)$ \\
\hline $5 \mathrm{~mm}$ Cube & $\mathrm{Y}=\left(1.54 \times 10^{8}\right) \mathrm{X}$ & $\mathrm{X}=22.71$ & $(4.7,2.7,4.8)$ \\
\hline $12 \times 12 \times 25 \mathrm{~mm}$ & $\mathrm{Y}=\left(1.33 \times 10^{9}\right) \mathrm{X}$ & $\mathrm{X}=2.63$ & $(11.8,12.9,11.7)$ \\
\hline $12 \mathrm{~mm}$ Cube & $\mathrm{Y}=\left(7.47 \times 10^{8}\right) \mathrm{X}$ & $\mathrm{X}=4.69$ & $(5.2,11.7,12.0)$ \\
\hline
\end{tabular}

Table 2.17: Simulation results for area force applied to a $1.5 \mathrm{~mm}^{3}$ cube, $5 \mathrm{~mm}^{3}$ cube, 12 $\mathrm{mm}^{3}$ cube and $12 \times 12 \times 25 \mathrm{~mm}^{3}$ platform structure. 
For applied pressures, simulated results indicate the $1.5 \mathrm{~mm}$ cubic platform is nine times stronger than the $5.0 \mathrm{~mm}$ cube and sixty times stronger than the existing $12 \times 12 \times 25$ $\mathrm{mm}^{3}$ platform structure when undergoing compressive pressures. For applied shearing pressures, the increase in strength of the $1.5 \mathrm{~mm}^{3}$ cube over the $5 \mathrm{~mm}^{3}$ and $12 \times 12 \times 25$ $\mathrm{mm}^{3}$ platform configurations are 9 and 74 times, respectively. Table 2.17 shows the bestfit linear equation determined from the MatLab ${ }^{\circledR}$, polyfit(), function and the maximum input pressure before fracture occurs which is an assumed output stress of $3.5 \mathrm{GPa}$ for each of the platform shapes mentioned above.

Shortening the long dimension of the $12 \times 12 \times 25 \mathrm{~mm}^{3}$ platform to become a 12 $\mathrm{mm}^{3}$ cube only slight increases to overall compressive and shear strengths by $2 \mathrm{MPa}$ when pressure is applied to the top side of the segment. This can be seen when comparing the maximum applied pressures in Table 2.17 for the two platform types.

Clearly, the reduction in platform volume dramatically increases the overall strength and enables the platform to be deployed in a greater number of applications. Also, the $1.5 \mathrm{~mm}^{3}$ cube configuration shown in Fig. $2.30(\mathrm{~d})$ has a total of $4.75 \mathrm{~mm}^{2}$ that can be used for microfabrication if one side of the wafer is utilized and $9.5 \mathrm{~mm}^{2}$ if both wafer surfaces are processed. Some processing area is lost because the planar component pieces require the peripheral interdigitated teeth features.

\subsection{Summary}

In this chapter, extensive three-dimensional finite-element modeling has been performed to verify the mechanical strength and viability of two types of platform 
connector techniques. The first is a Hook-Hole combination where a flexible micromachined Hook feature, similar to a fixed-end cantilever beam, is completely inserted into a Hole feature to create an interlocking structure. Dynamic FEM results show that the maximum amount of stress in the Hook feature occurs when the tip of the Hook is within the Hole feature and remains a constant value until returns to zero after snap-locking into place.

Full platform structural simulations were also performed for $0.5 \mathrm{~mm}, 1.0 \mathrm{~mm}$ and 2.0mm long interdigitated teeth implementations. This is the second platform connection method investigated. Results indicate that the longer teeth design results in a mechanically stronger platform when compressive and shear pressures are applied to it. Other applied point forces and line forces showed no significant difference in platform mechanical strength when comparing the different interdigitated teeth designs.

Simulations were also performed on full platform assemblies that implemented the Hook-Hole and interdigitated teeth connection strategies. Replacing the Hook-Hole features with simple rectangular, interdigitated teeth features increases the overall mechanical strength of the platform by three to five times depending on the type of applied pressure. Furthermore, since all the platform pieces have identical periphery features, the variance in the generated stresses is reduced for applied pressure, point and edge forces.

Finally, analyzing the $25 \times 25 \times 12 \mathrm{~mm}^{3}$ platform with $1 \mathrm{~mm}$ interdigitated teeth the following is noted. The applied pressure simulation results indicate the platform is more resistant to fracture from compressive verses shear forces, on average, by $40 \%$. The 
range of maximum input pressure before fracture for the compression and shear scenarios are (3.05 - 4.30) MPa and (2.63 - 2.97) MPa, respectively. In both the compression and shear force scenarios, the End cap/Connector platform segment proved to be the weakest compared to the larger Top and Side platform segment pieces. However, the simulation results indicate the more square the platform segment is the less variability in the generated stress when comparing between compressive and shear area applied forces.

In all the applied point force simulations, the force was applied directly at the center and at a normal angle to the platform segment's surface. For the applied point force simulations, the results show the platform is slightly stronger for compressive than shear forces, on average, by $1.6 \%$. The maximum input force range before fracture for the compression and shear scenarios are $(68.90-82.16) \mathrm{N}$ and $(70.56-82.55) \mathrm{N}$, respectively. Similar to the applied pressure scenarios mentioned above, the more square the platform segment the less variability between compression and shear generated stresses for a given input point force. Finally, in both compressive and shear force scenarios, the End cap/Connector face required the highest input point force before fracture.

For the edge force simulations, the forces were applied evenly across the platform edge at an angle of $45^{\circ}$ from the normal surface angles of the platform sides that form the edge. For the applied edge force simulations, the results found show the platform is stronger for compressive than shear forces, on average, by $31 \%$. The range of maximum input force range before fracture for the compression and shear scenarios are $(103,245$ $114,754) \mathrm{N} / \mathrm{m}$ and $(77,263-92,105) \mathrm{N} / \mathrm{m}$, respectively. For both the Top-Side edge compression and shear force simulation results, the failure location occurs in the same 
location on the End cap/Connector segment. This failure location is at the upper corner of the hole where the external 8-pin connector extents out of the platform. Therefore, similar to eliminating the Hook-Hole features from the platform periphery, these other hole features weaken the platform mechanically and should be avoided if possible.

Strain is defined as the displacement gradient of particles within the material. Stress is equivalent to the strain multiplied by the elasticity (or compliance) matrix which is function of a material's Young's Modulus and the Poisson's ratio values. Therefore, to reduce the stress within a given material, one must reduce the structural deformation when applied pressures and forces are exerted. This observation holds true for all the simulation results described above.

Mechanically stronger configurations resulted when the platform approached resembling a single, continuous, hollow, piece of silicon. For example, the $2 \mathrm{~mm}$ long teeth proved to produce the most structurally sound platform compared to the other, smaller teeth designs. Also, when the Hook-Hole features were removed from the platform and replaced with the interdigitated teeth, it resulted in a significant increase in platform mechanical strength. The maximum stress points always occurred at points at the Hole boundaries for the pressure simulations. This supports the above mentioned observation because the material is more likely to be deformed than in structure without the Hole features. The platform configurations that reduced the amount of material displacements resulted in mechanically stronger structures. 


\section{Chapter 3}

\section{Platform Microfabrication}

The 3-D platform is fabricated using simple, well defined IC and MEMS processing technologies. All the platform components (i.e., male, female and end caps) are created on a single silicon wafer which simplifies the fabrication process. The fabrication process is reviewed in the next section. Further details about the process can be found in Appendix A. The fabrication process steps were performed at Case Western Reserve University's MicroFabrication Lab (MFL) unless otherwise noted.

\subsection{Hook-Hole Microfabrication Processs}

The process begins with a 4-inch, double-sided polished, (100) silicon wafer with a thickness of $250 \mu \mathrm{m} \pm 10 \mu \mathrm{m}$. The wafers undergo a thermal oxide process step to create a silicon dioxide layer with a thickness of $3000 \AA$ (Fig. 3.1(a)). This oxide serves as the electrical isolation layer from the bulk silicon wafer.

Next, AZ1813 positive photoresist was spun on and exposed to the metal pattern mask, then developed to prepare the openings for metal deposition. Four wafers were then delivered to the NASA Glenn Research Facility where $100 \AA$ of titanium (Ti) was evaporated onto the wafers immediately followed by $3000 \AA$ of gold (Au) (Fig. 3.1(b)). The metal lift-off procedure was performed at the CWRU MFL followed by PECVD to deposit a $3000 \AA$ silicon dioxide layer used as an encapsulation layer to protect the metal traces (Fig. $3.1(\mathrm{c}))$. 
AZ9260 positive photoresist was then spun on the frontside of the wafers and exposed with the photomask to etch through the PECVD silicon dioxide using a RIE technique to expose the metal features, which are used as soldering pads during assembly (Fig. 3.1 (d)). The photoresist is then stripped away and the wafer thoroughly cleaned.

Another AZ9260 positive photoresist layer is spun on wafers and patterned with the photomask for front side RIE/DRIE processing to first remove the $6000 \AA$ of total field silicon dioxide with the RIE process (Fig. 3.1 (e)). Using the existing photoresist pattern on the wafer, a DRIE process is performed to etch $200 \mu \mathrm{m}$ into the bulk silicon to create the hook and hole features around the periphery of the platform pieces (Fig. 3.1 (f)). The photoresist is then stripped away and the wafer thoroughly cleaned.

AZ9260 positive photoresist is spun on the backside of the wafer. Using backside alignment the photoresist is then patterned with the appropriate photomask for backside DRIE. The patterned wafer is placed on a carrier wafer, and a DRIE process is performed to release the individual platform components from the bulk silicon wafer (Fig. $3.1(\mathrm{~g})$ ). The processed wafer is then placed in a beaker of acetone overnight to release the device wafer from the carrier wafer and also to retrieve all the individual platform components.

The DRIE etch profile was determined from scanning electron microscopy (SEM) photos to have the following characteristics: sidewall angle $=90^{\circ}$, etch rate $=2.98$ $\mu \mathrm{m} / \mathrm{min}$, scallop $=200 \mathrm{~nm}$ and an undercut of $406.7 \mathrm{~nm}$. 

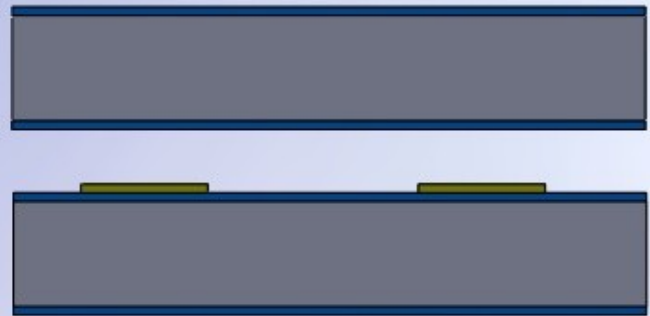

(b)

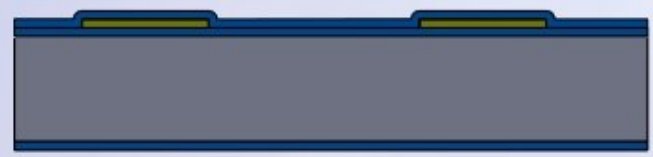

(c)

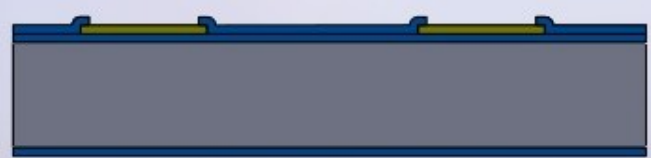

(d)

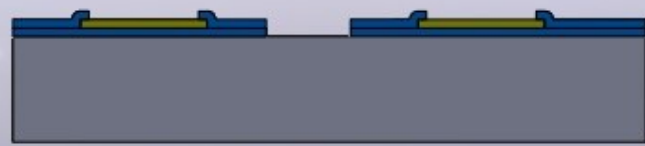

(e)

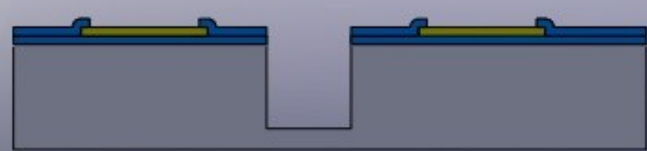

(f)

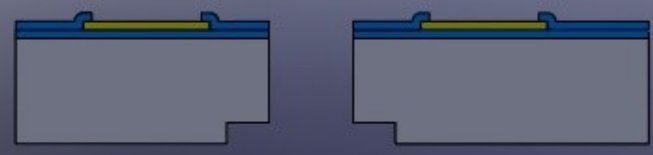

(g)

Figure 3.1: Fabrication steps for the Hook-Hole platform : (a) Thermal silicon dioxide; (b) Ti/Au metal deposition; (c) PECVD silicon dioxide; (d) silicon dioxide window RIE; (e) silicon dioxide field RIE; (f) front-side DRIE and (g) backside DRIE. 


\subsection{Interdigitated Teeth Microfabrication Process (i.e. No Hook-Hole Features)}

The fabrication details are nearly identical those described above. However, two metal interconnect layers are used to account for any potential system-level signal routing complexity. This adds more processing steps and an additional photomask for a total of five photolithography steps. As previously mentioned, the Hook-Hole features are removed which simplifies the DRIE processing but adds additional steps in the assembly process as discussed in Chapter 4. Figure 3.2 summarizes the microfabrication flow for the interdigitated teeth platform design.

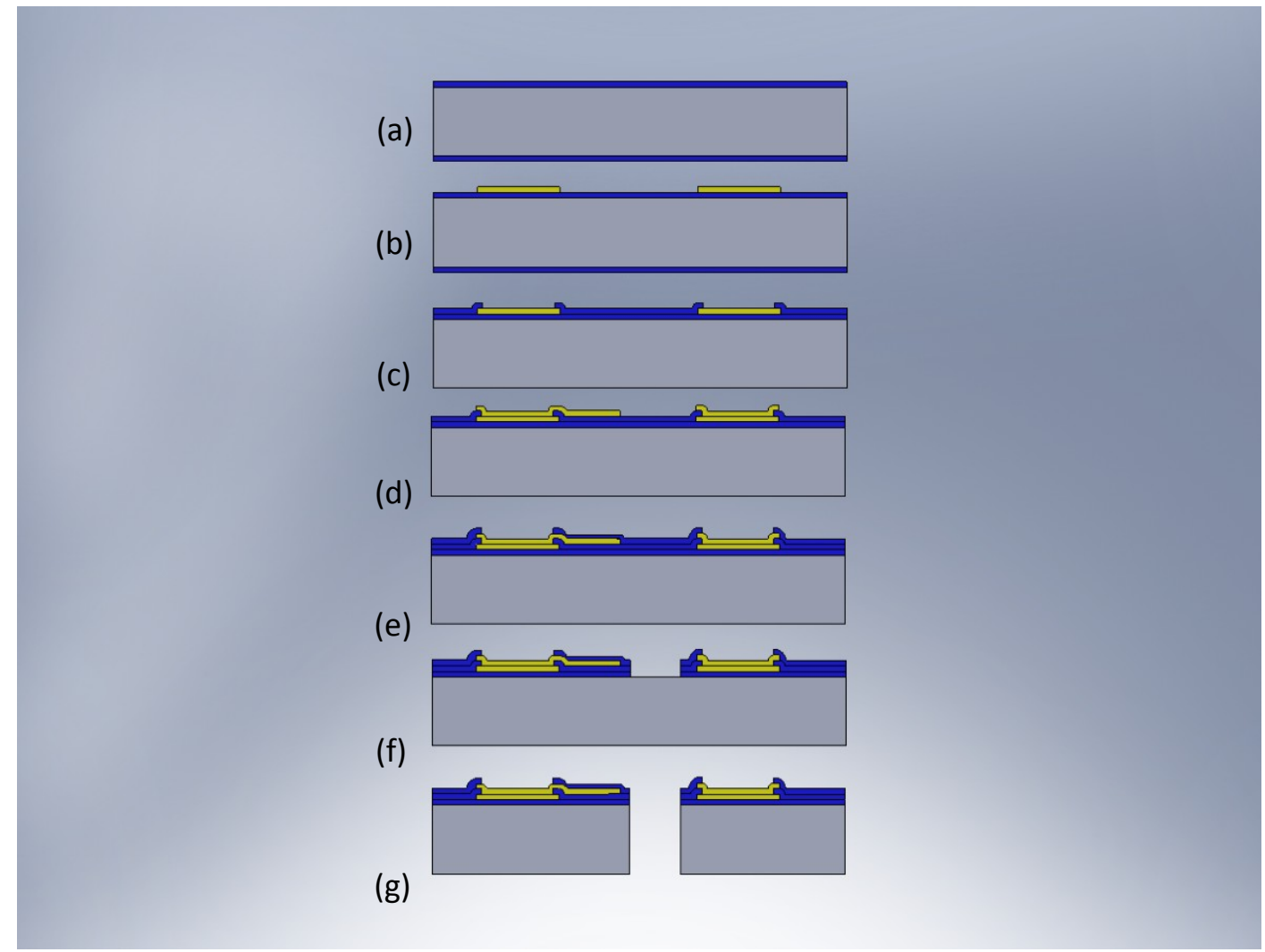

Figure 3.2: Interdigitated teeth platform fabrication process: (a) thermal oxide, $3000 \AA$; (b) metal deposition, $100 \AA / 3000 \AA$ (Ti/Au); (c) PECVD TEOS oxide deposition $(5400 \AA)$

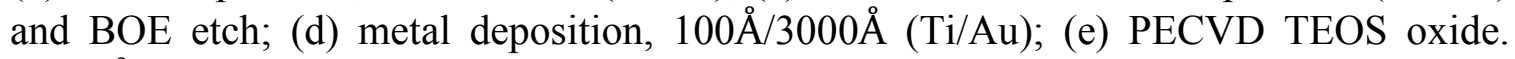
$(5400 \AA ̊)$ and BOE etch; (f) Field Oxide BOE etch; and (g) Frontside DRIE etch (250 $\mu \mathrm{m})$. 
All silicon wafer processing was performed at the Case Western Reserve University, except the metal deposition steps which were performed at the NASA Glenn Research Facility in Cleveland, $\mathrm{OH}$. The details for all the process steps can be found in Appendix A. Silicon wafers (111), $250 \mu \mathrm{m} \pm 10 \mu \mathrm{m}$-thick, double-sided polished were used to create $1 \mathrm{~mm}$ length, interdigitated teeth platform structures.

Each 4-inch silicon wafer is able to create two complete platform structures as shown in Fig. 3.3.

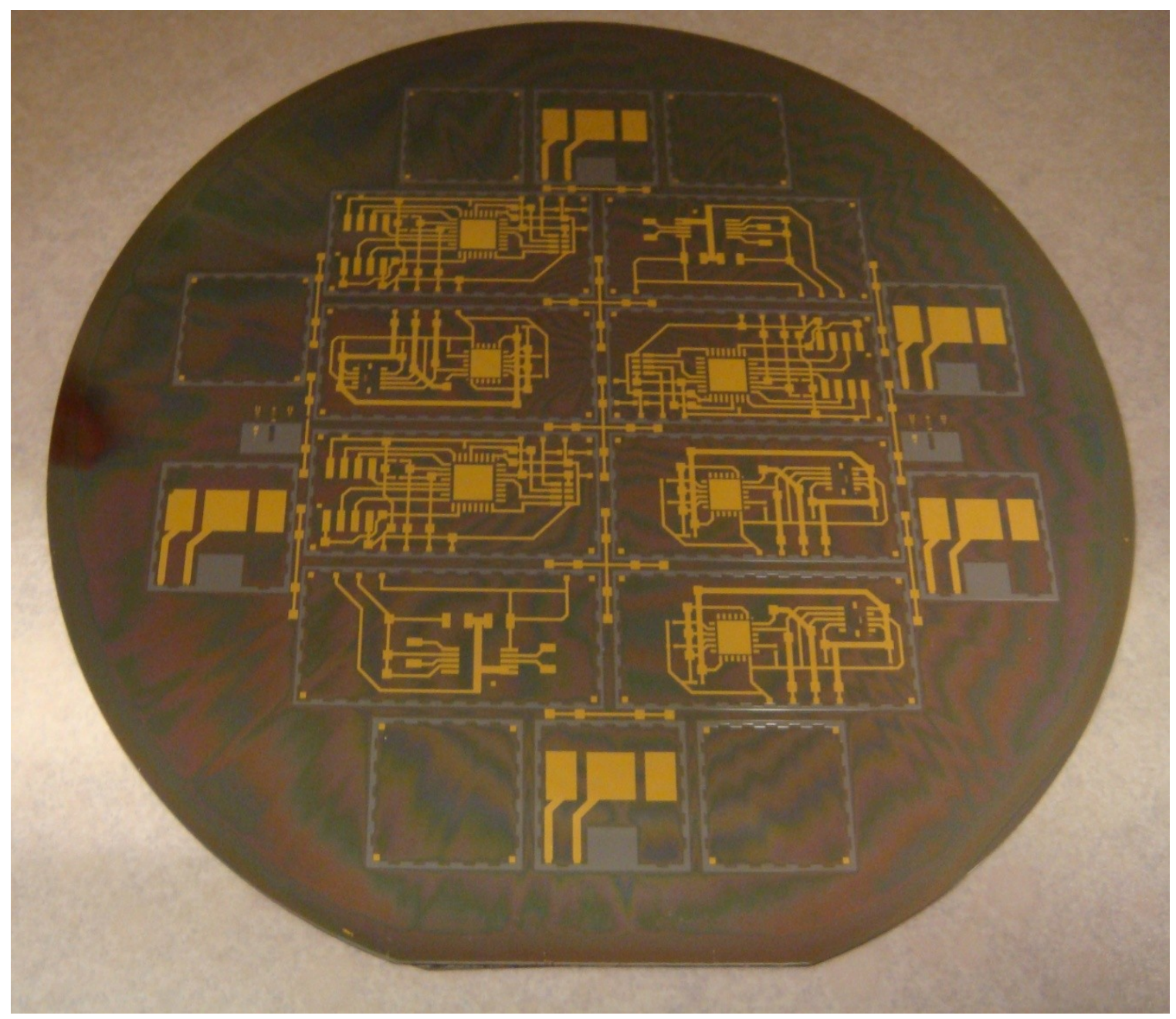

Figure 3.3: Interdigitated teeth platform - 4-inch wafer before DRIE processing. 


\section{Chapter 4}

\section{Platform Assembly Procedures}

This chapter discusses the assembly procedure for both types of platform connector techniques (i.e., Hook-Hole and interdigitated teeth). In both cases, there are three key processes to create a mechanically and electrically sound and functional threedimensional platform. The first is creating stable solder connections for the passive and integrated-circuit (IC) components placed on the platform segment pieces. The next is mechanically assembling the platform structure to maintain a three-dimensional shape. Finally, mechanically and electrically stable orthogonal solder joints must be created to join the different platform segment pieces together.

First, there is a brief discussion on the different solder and assembly jig materials investigated as well as soldering methods. This is followed by a discussion on the methods and materials used for each of the platform connector types.

\subsection{Investigating Different Solder and Assembly Jig Material Choices}

A key element to creating a successful platform is the ability to route electrical signals within the structure. To route signals on the sidewalls and between different component pieces of the cube, requires the ability to create out-of-plane, orthogonal electrical connections where the two component pieces join together. In addition, creating sound solder connections from the passive circuit components and the ICs to the individual platform segments pieces is also important. 
To achieve these stable solder connections, different solder material types were investigated including pastes, ribbons and prefabricated spheres as shown in Figure 4.1.

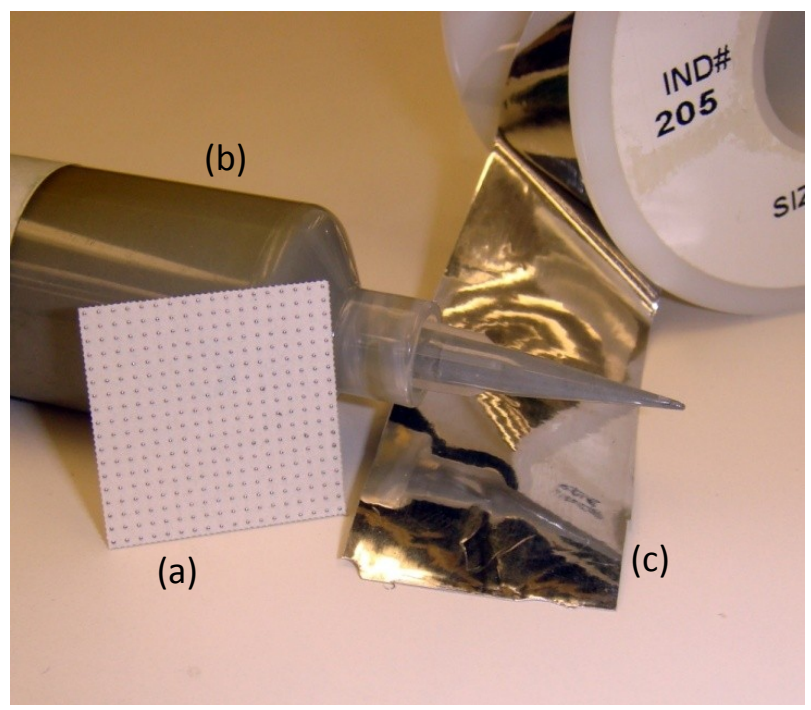

Figure 4.1: Different solder types investigated: (a) prefabricated spheres; (b) paste; and (c) ribbon.

\begin{tabular}{|c|c|c|c|c|c|c|c|}
\hline Solder & Composition & Type & ${ }^{\circ} \mathrm{C}$ & ${ }^{\circ} \mathrm{C}$ & $\mathrm{g} / \mathrm{cm}^{3}$ & $\begin{array}{c}\text { Tensile } \\
\text { SPa }\end{array}$ & $\begin{array}{c}\text { Electrical } \\
\text { Conductivity } \\
\% \text { IACS }\end{array}$ \\
\hline 256 & $96.5 \mathrm{Sn3} 3.0 \mathrm{Ag} 0.5 \mathrm{Cu}$ & Paste & 220 & 217 & 7.40 & 49.0 & 13 \\
\hline 2 & $80 \ln 15 \mathrm{~Pb} 5 \mathrm{Ag}$ & Paste & 154 & 149 & 7.85 & 17.5 & 13 \\
\hline 205 & $60 \mathrm{In} 40 \mathrm{~Pb}$ & Paste & 181 & 173 & 8.52 & 28.6 & 7 \\
\hline 206 & $60 \mathrm{~Pb} 40 \mathrm{In}$ & Paste & 231 & 197 & 9.30 & 34.5 & 5.2 \\
\hline 106 & $63 \mathrm{Sn} 37 \mathrm{~Pb}$ & Sphere & $183 \mathrm{E}$ & 183 & 8.40 & 51.7 & 11.5 \\
\hline
\end{tabular}

Table 4.1: Solder compositions investigated for platform electrical connectivity. 
Table 4.1 is a summary of all the solder compositions and forms tested along with their physical and electrical properties. All the solder types under investigation had electrical conductivities ranging from 5.2\% to 13\% IACS (International Annealed Copper Standard $=58 \times 10^{6} \mathrm{~S} / \mathrm{m}$ ) which results in low solder joint resistivity.

Another key property is having a large tensile strength to secure the platform pieces together. Finally, the most critical property for platform assembly is to have two solder types that do not have overlapping solidus-liquidus ranges. Solidus is defined as the temperature where the solder transitions from a solid to slurry. Liquidus is defined as the temperature at which the solder is completely liquidized. The higher liquidus temperature solder is used first to attach all the passive electronic components and ICs to the platform pieces. Then the lower liquidus temperature solder is used to make the orthogonal electrical connections. This process allows the orthogonal joints to be created without reflowing the existing electrical component solder joints.

To achieve this orthogonal electrical connection, specific solder pads should be designated in both platform designs. Figure 4.2 shows the orthogonal solder joint for fabricated end cap and male component pieces which have $330 \times 330 \mu \mathrm{m}^{2}$ bond pad features that align to each component connection edge. These are used as soldering locations to electrically connect the component pieces together. Also $480 \times 480 \mu \mathrm{m}^{2}$ metal features are present along the top and bottom edge of the platform components to act as additional soldering points for added mechanical strength if needed. However, both of the platform connector methods were found to be sufficient. 


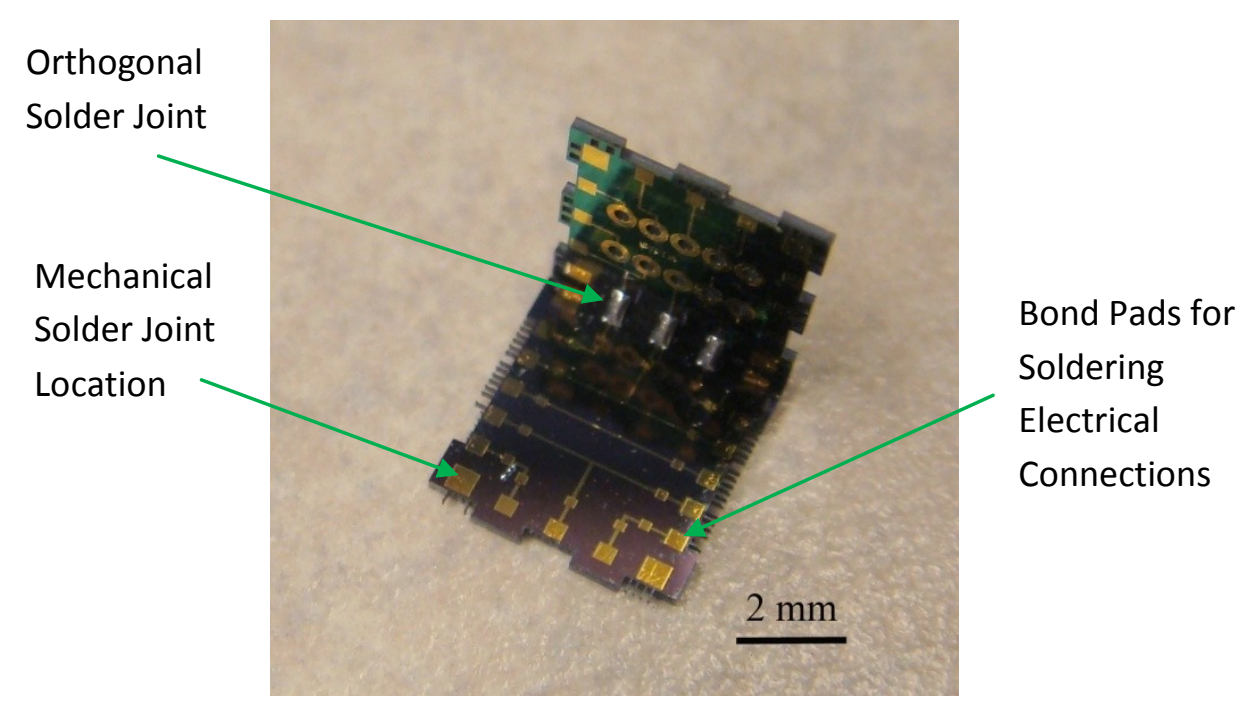

Figure 4.2: Orthogonal solder joints connecting male and end cap components.

Soldering the platform component together piece by piece and by hand proved to be a challenging process as numerous trials were performed to achieve consistent and successful solder joint connections. The variables used in the experiments were the different solder compositions, solder types (see Fig. 4.1), jig assembly material (see Fig. 4.3) and finally the soldering method (i.e., hotplate, high temperature furnace and infrared (IR) heater). 


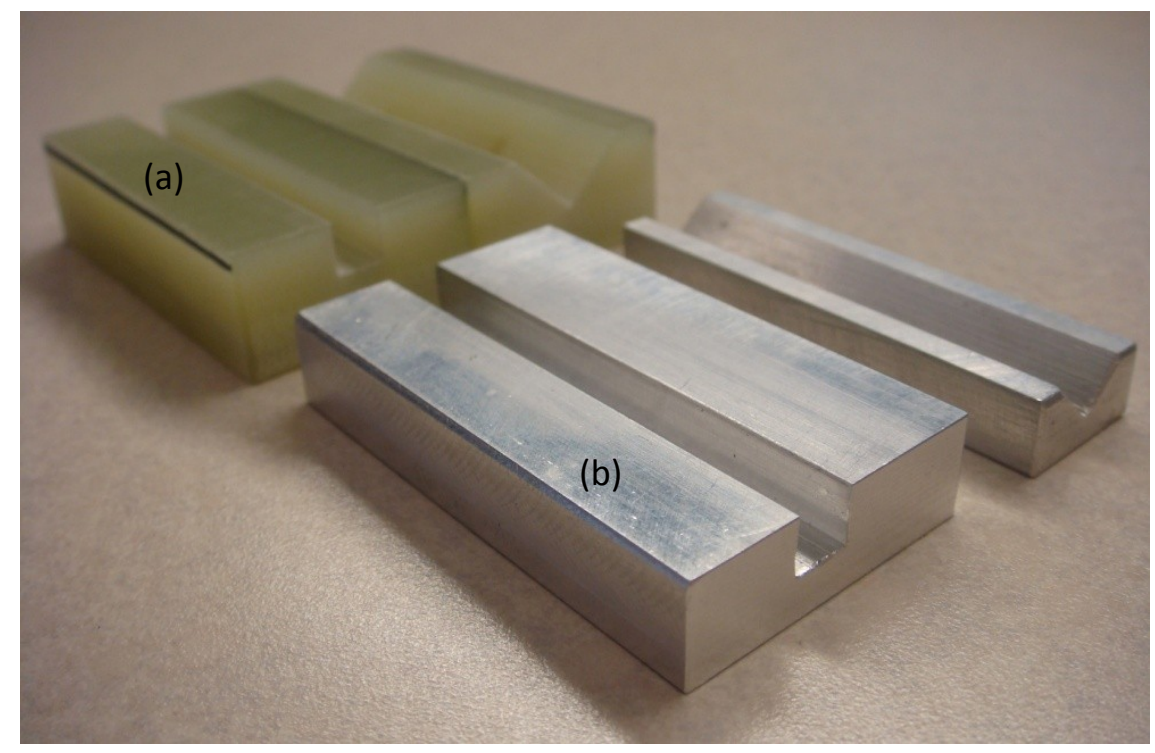

Figure 4.3: Jig material tested for platform assembly: (a) FR-4; and (b) aluminum. (Macor ${ }^{\mathrm{TM}}$ material is not shown in this figure).

When soldering the platform pieces together, the 15 mil diameter preformed solder spheres resulted in the best solder joints as opposed to the solder paste and ribbon; having a consistent volume of solder dramatically increased the joint fabrication success rate. Comparing the results after using various jig materials such as aluminum, FR-4 (i.e., Fire-Resistant-4, printed circuit board material) and Macor ${ }^{\mathrm{TM}}$ proved FR-4 to be the best assembly jig material to hold the platform pieces during the soldering process. The aluminum and Macor ${ }^{\mathrm{TM}}$ assembly jigs acted like large heat sinks and would prevent the solder location from rapidly heating which resulted in poor, unconnected solder joints between platform pieces. Finally, a hotplate proved to be the best method for soldering the platform pieces together. The time, uniform heating, and temperature could be easily controlled and monitored during the soldering process in addition to visual monitoring the solder joint formation. 
Based on the criteria listed above and numerous experiments for soldering the platform's integrated circuits and passive devices, the $60 \% \mathrm{~Pb}-40 \%$ In solder paste is selected with its higher liquidus temperature of $231^{\circ} \mathrm{C}$. In addition, the $60 \mathrm{~Pb} 40 \mathrm{In}$ solder paste is known to minimize gold leaching which is advantageous for this platform since

all the electrical routing and bond pads are fabricated using gold. The $63 \% \mathrm{Sn}-37 \% \mathrm{~Pb} 15$ mill diameter solder spheres is selected for the electrical and mechanical connections between platform pieces. The $63 \mathrm{Sn} 37 \mathrm{~Pb}$ solder spheres have a $48^{\circ}$ lower liquidus temperature of $183^{\circ} \mathrm{C}$ compared to the $60 \mathrm{~Pb} 40 \mathrm{In}$ solder paste.

\subsection{Platform Assembly with Hook-Hole Features}

\subsubsection{Female and Male Component Assembly with Hook-Hole Features}

Male Components were fabricated with the identical Hook features previously described. The female components had three different Hole widths: $160 \mu \mathrm{m}, 170 \mu \mathrm{m}$ and $180 \mu \mathrm{m}$. Some female components on the wafer had modified peripheral features to help verify that the Hooks would pass through the holes, snap into place and join the two male and female component edges firmly together. These modified features were simple holes that were etched during the front-side DRIE process step to mimic a cross-sectional view of the actual female component Holes. Figure 4.4 is a close-up of the test features as the Hook is being advanced into the test Hole structure. 


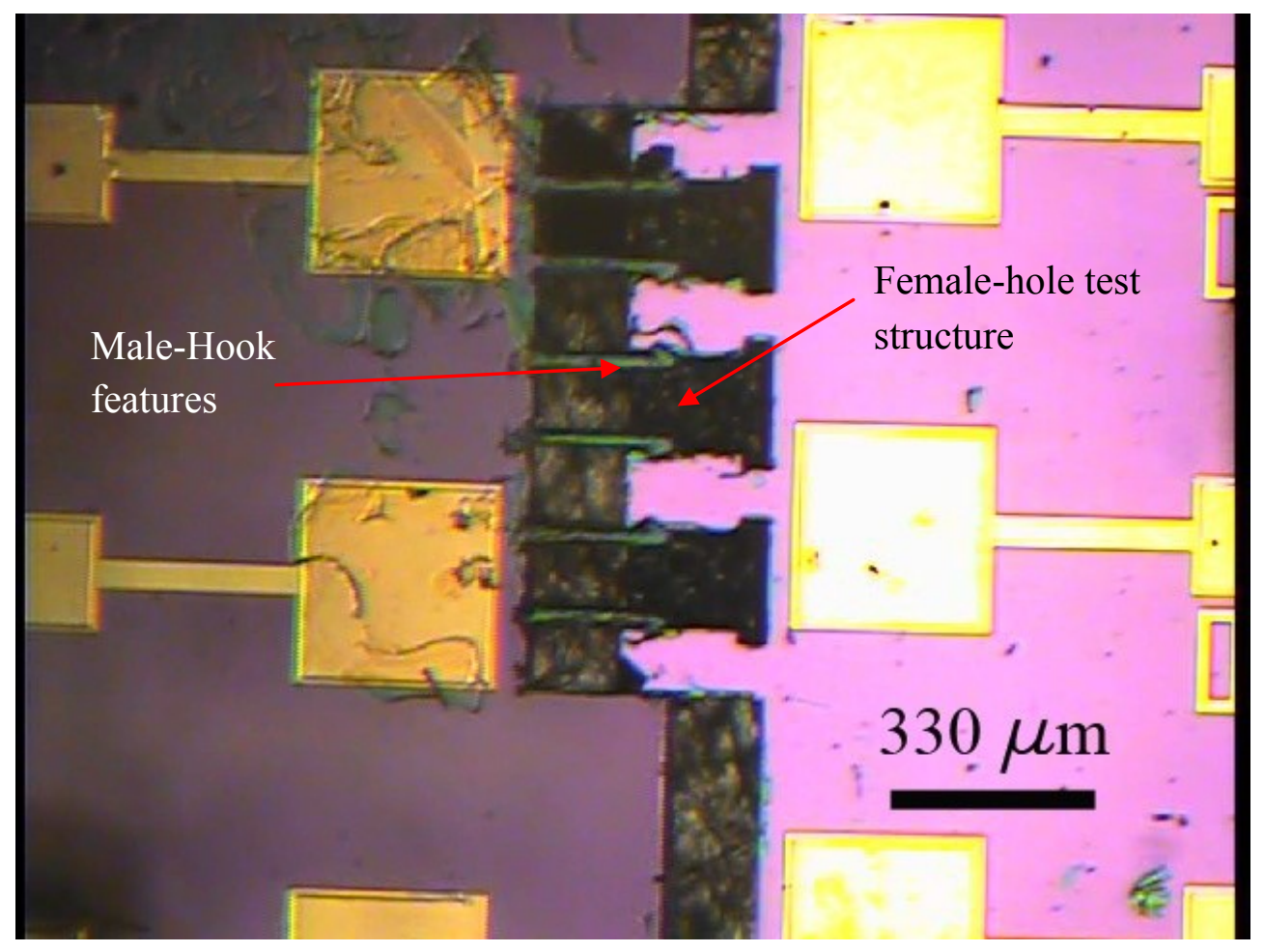

Figure 4.4: Hook test structure (left) being inserted into Hole test structures (right) to examine component assembly.

Repeated trials of inserting the hook features into the test hole features resulted in very few instances where a Hook broke, even for the smallest $160 \mu \mathrm{m}$ wide Hole features. Once the Hook-Hole concept was verified, a complete platform cube was assembled purely based on the Hook-Hole connections. No additional adhesives or processing was required to form the cube structure shown in Fig. 4.5. 


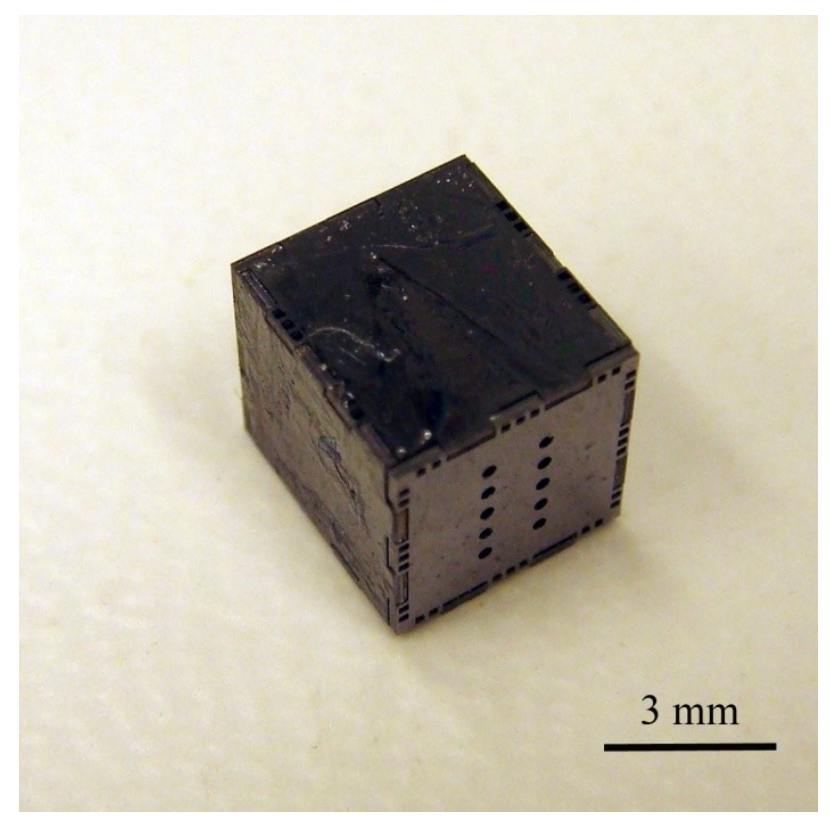

Figure 4.5: Fully-assembled cube structure using only Hook-Hole connectors.

The following describes the procedure to mechanically connect the Hook-Hole platform together as shown in Figure 4.5. First, the two male segment pieces (A) are snapped into the base female segment (B) as shown in Figure 4.6 to create a "U" shape.

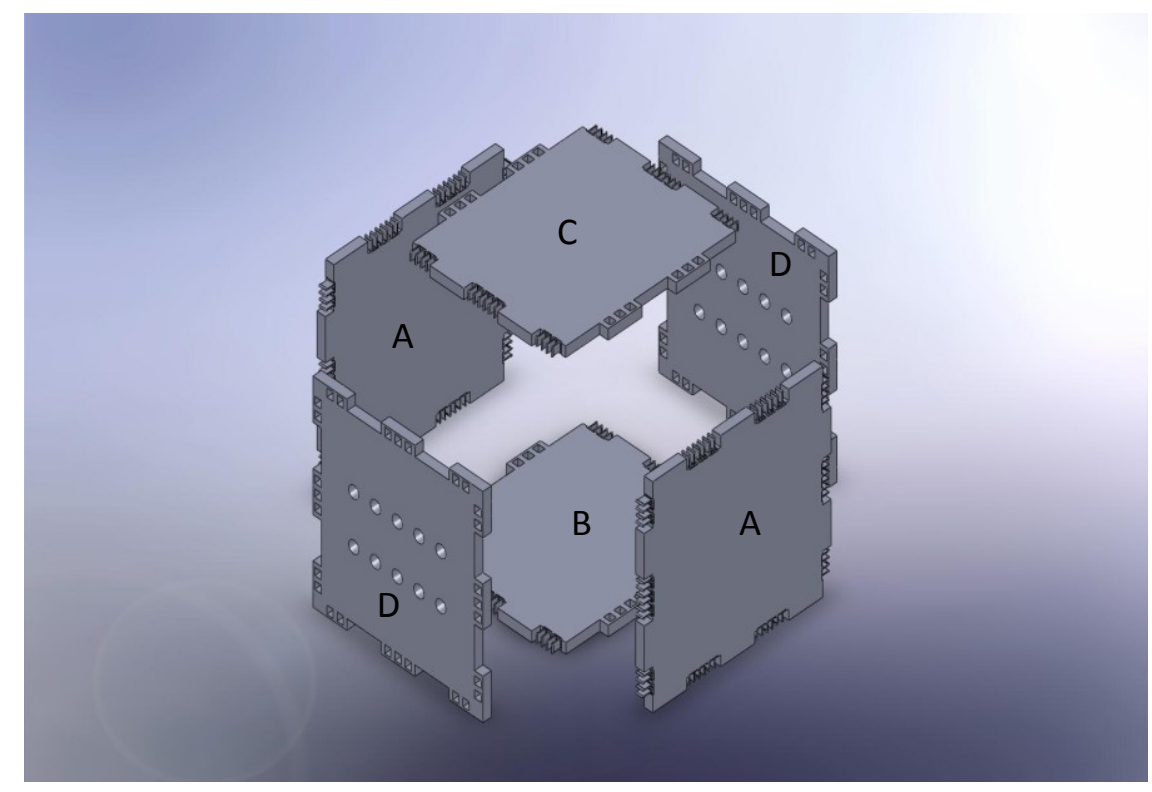

Figure 4.6: Hook-Hole mechanical assembly steps to create the platform structure. 
Next, the top female segment (C) is snapped into the top edges of segments (A) to create a doughnut shaped structure. Finally, the end cap components (D) are snapped into place to complete the enclosed platform structure. No additional adhesives are needed for the platform to maintain its three-dimensional shape.

Creating the platform electrical connections is identical for both types of connector methods and is discussed in the next section.

\subsection{Platform Assembly with Interdigitated Teeth Features}

\subsubsection{Integrated-Circuit and Passive Component Soldering Process}

To create a fully-assembled platform with interdigitated teeth, the first step is to solder the integrated circuits (ICs) and passive components to their respective platform pieces before soldering the side pieces together. For each of the platform segments, the process of soldering the electrical components begins with the platform segment placed on a bare, $250 \mu \mathrm{m}$ thick, silicon wafer (Fig. 4.7(a)). Using a needle, a small amount of 60 $\mathrm{Pb}-40 \mathrm{In}$ solder paste is manually applied to the passive component bond pad sites (Fig. 4.7(b)). Using tweezers, the passive components are manually placed on the bond pad locations (Fig. 4.7(c)). Solder is then placed on the IC bond pad sites (Fig. 4.7(d)). ICs are then hand placed (Fig. 4.7(e)). Tweezers are then used to pick up the bare wafer and place it on a $230^{\circ} \mathrm{C}$ hotplate (Fig. $4.7($ f) ) for 20 seconds or until the solder reflows (Fig. 4.7(g)). Tweezers are again used to carefully remove the bare silicon wafer from the hotplate and set on a table-top to cool (Fig. 4.7(h)). Electrical checks are then performed to verify the soldering process was successful for all the passive and IC components. 


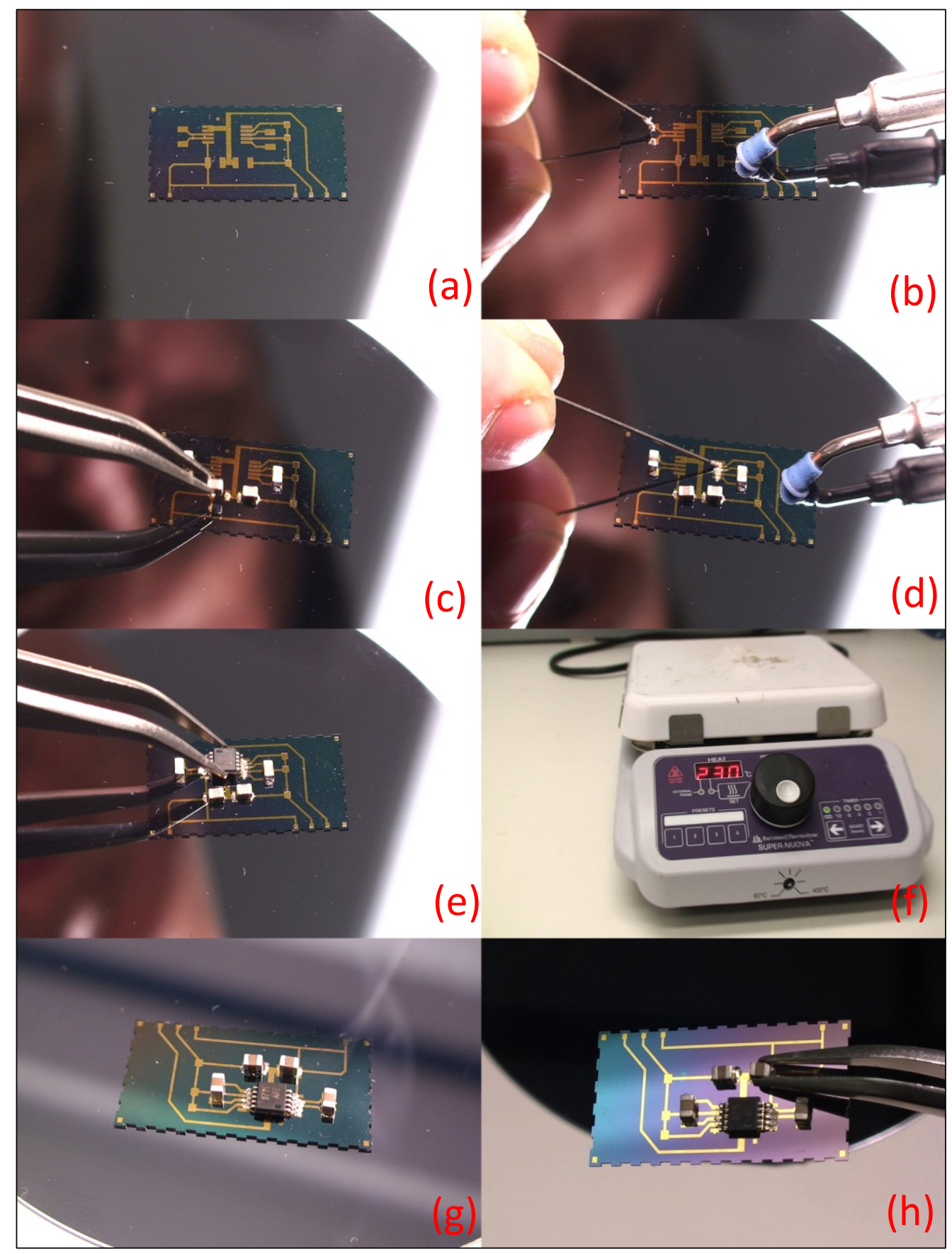

Figure: 4.7: Procedure to solder passive and IC components to the platform segment pieces: (a) segment placed on silicon handle wafer; (b) solder paste applied to passive bond pad locations; (c) passive components placed on platform segment; (d) solder paste placed on IC bond pad locations; (e) IC components hand placed on platform segment; (f) tweezers used to place wafer on hotplate; (g) wait until solder reflows $(20 \mathrm{sec})$; (h) tweezers used to pick up completed platform segment after removing the silicon wafer from the hotplate and allowed to cool for a minute. 
The process is repeated for all integrated circuit, passive and connector components as shown in Fig, 4.8.

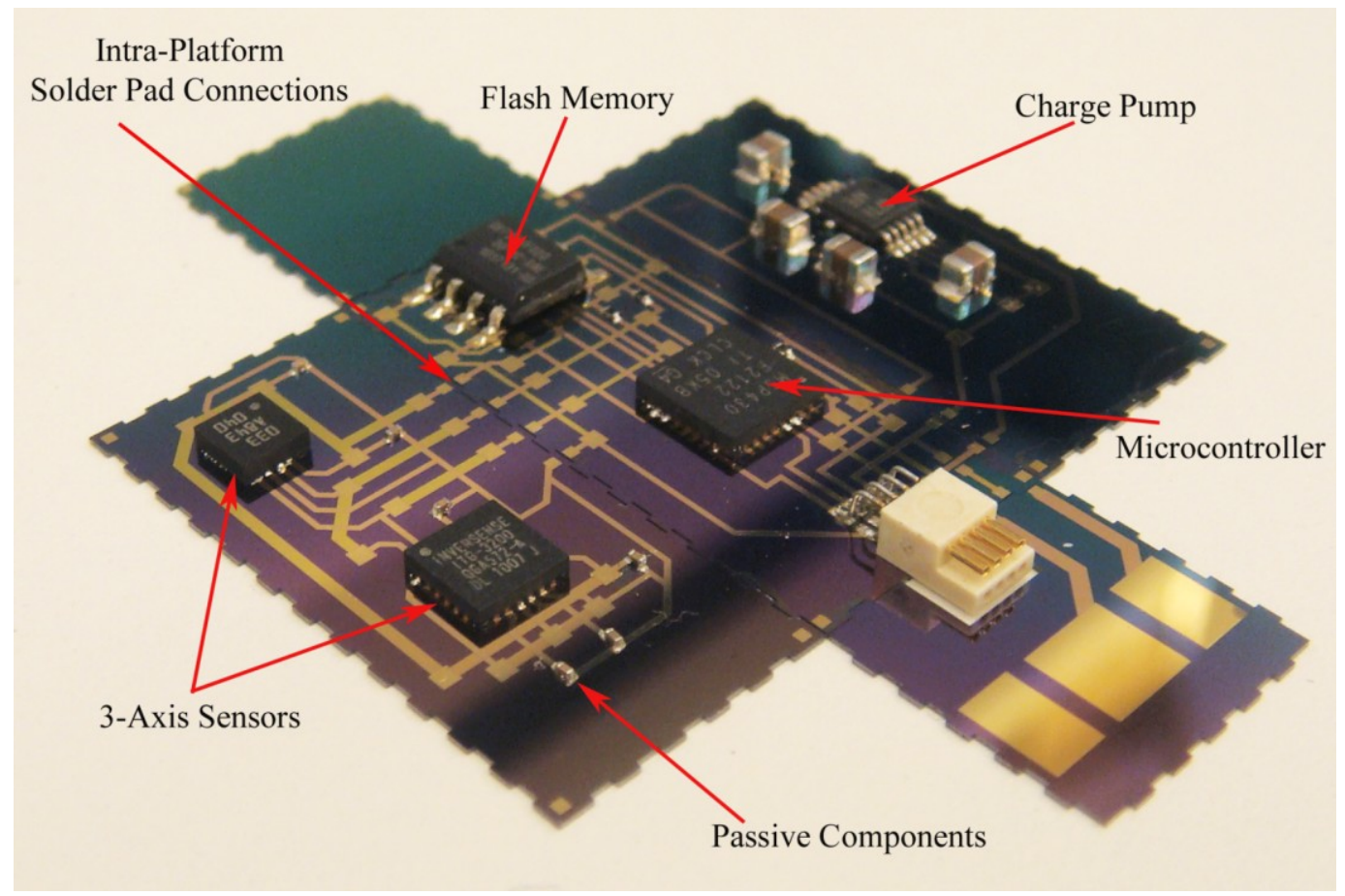

Figure 4.8: Completed platform segments with all passive and IC components soldered.

\subsubsection{Process for Assembling Platform Segment Pieces Together}

As shown in Fig. 4.3(a), pieces of FR-4 material were machined to create assembly jigs to aide in the assembly process. One jig has a triangle-machined crosssection to provide a $90^{\circ}$ angle to create a single seam between two platform pieces.

Using the FR-4 assembly jig, two platform segments are jointed together (Fig. 4.9(a)) and small drops of Silastic ${ }^{\mathrm{TM}}$, a silicone elastomer, is placed at the seam created by the two segments (Fig. 4.9(b)). The FR-4 jig is then placed under a $100^{\circ} \mathrm{C}$, infra-red heat source for $15 \mathrm{~min}$ to cure the Silastic ${ }^{\mathrm{TM}}$ (Fig. 4.9(c)). 


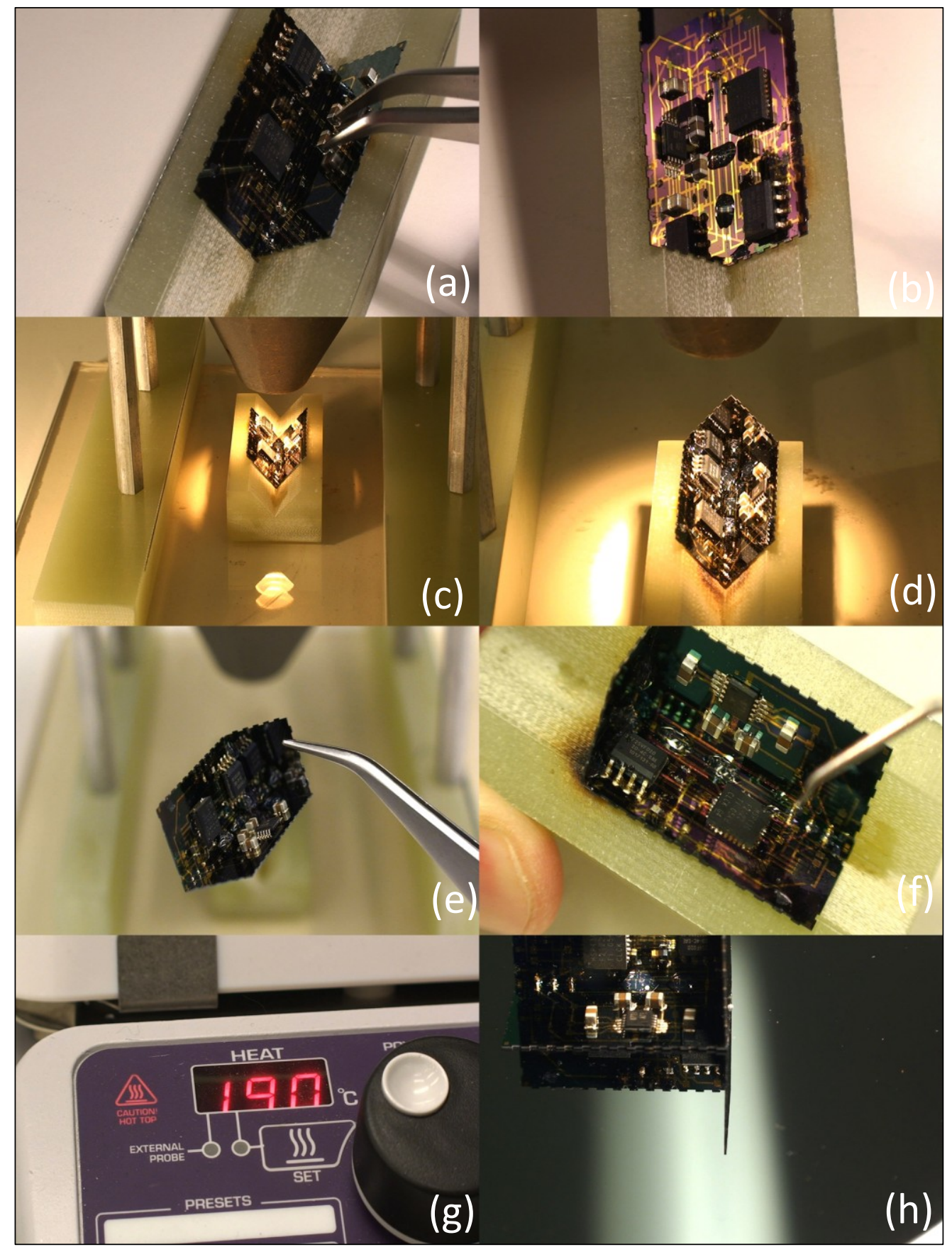

Figure 4.9: Process to assemble interdigitating teeth platform: (a) join two segments together on the FR-4 jig; (b) place drops of Silastic ${ }^{\mathrm{TM}}$ at the seam created by the two segments; (c) place jig under IR-heater to cure Silastic ${ }^{\mathrm{TM}}$; (d) repeat process to attach an end cap segment to the platform; (e) assembly is halfway complete; (f) place preformed solder spheres with TACFLUX ${ }^{\mathrm{TM}}$ on orthogonal solder pad locations; (g) place platform on silicon wafer then on hotplate; (h) wait until the solder spheres reflow and orthogonal solder joints are created (upper left-hand corner). 
The same three steps are repeated to attach an end cap platform segment (Fig. 4.9(d)) to make sure the two platform segments are orthogonal to each other. This is critical when the orthogonal solder joints are created. The three-dimensional platform structure is now halfway assembled (Fig. 4.9(e)).

The platform structure is then placed back in the FR-4 jig and TACFLUX ${ }^{\mathrm{TM}}$ is applied with a needle to the bond pad sites for the four orthogonal solder joint locations. Then, 15 mil diameter, preformed solder spheres are placed on each bond pad site, eight total, such that adjacent solder spheres are touching each other to form a connection across the segment pieces (Fig. 4.9(f) indicated by needle tool). The platform structure is then placed on a bare, $250 \mu \mathrm{m}$-thick, silicon wafer. Using tweezers, the wafer is placed on a $190^{\circ} \mathrm{C}$ hotplate (Fig. $4.9(\mathrm{~g})$ ) for 20 seconds or until the solder spheres reflow and form the orthogonal, intra-platform solder joints (Fig. 4.9(h) upper-left corner). Placing the platform on the hotplate does not damage or affect the adhesive properties of the Silastic ${ }^{\mathrm{TM}}$ elastomer. Using tweezers again, the silicon wafer is removed from the hotplate and allowed to cool for one minute before handling the platform structure.

The assembly process continues by soldering the battery holder terminals to the platform with the $60 \mathrm{~Pb}-40 \mathrm{In}$ solder paste, followed by attaching the connector/end cap segment to the platform structure with Silastic ${ }^{\mathrm{TM}}$ as previously mentioned. Following this step, the final segment is attached per the method previously described by creating the orthogonal solder joints between the two platform segments. Figure 4.10 shows the platform structure with the integrated battery holder and an unattached segment and next to it a fully-assembled platform. The Top platform segment is fixed into place by using a 
small drops of cyanoacrylate glue placed on each of the four edges. Removal of the Top segment is done by placing the top side of the platform in a small dish of acetone for five minutes to dissolve the glue.

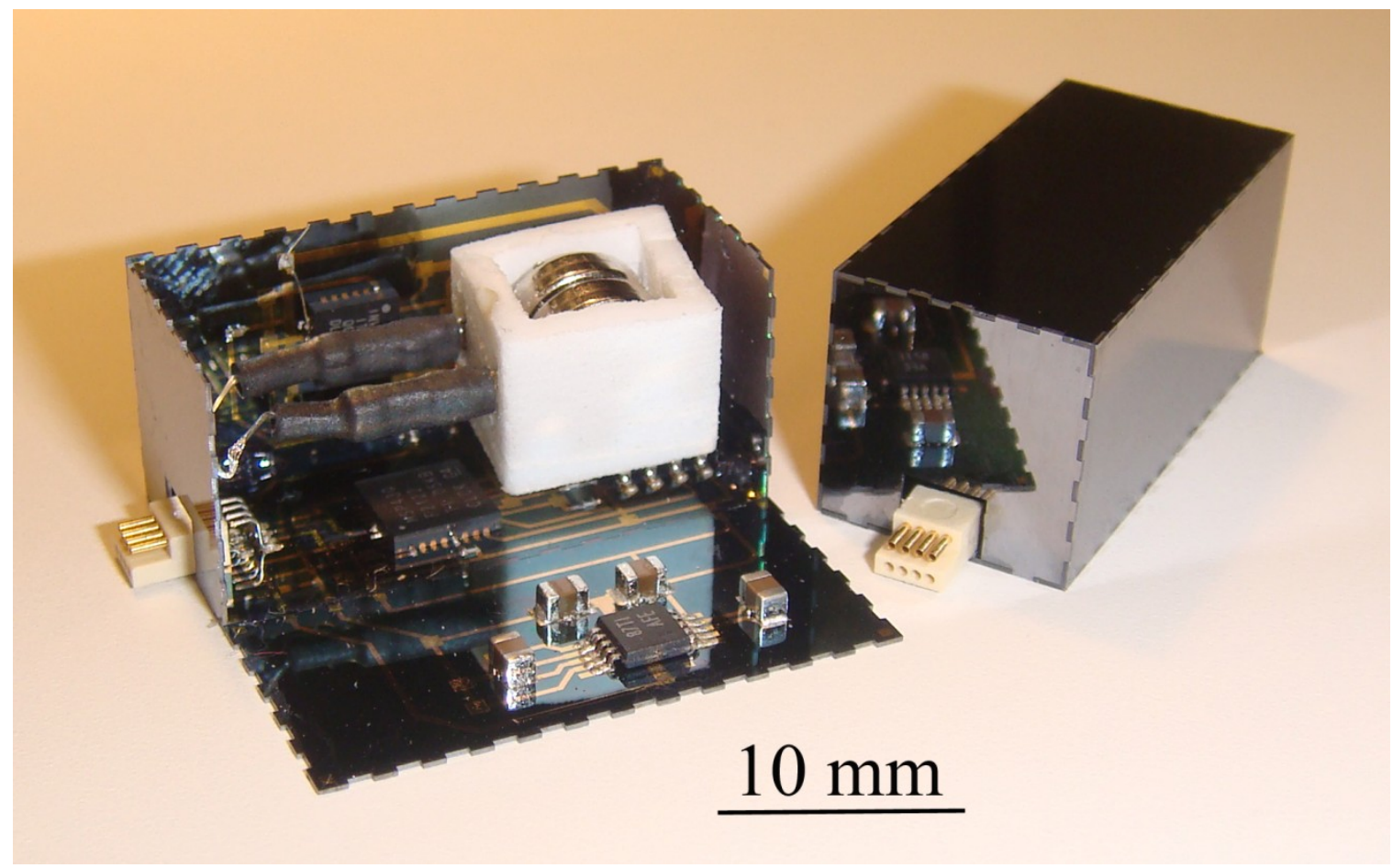

Figure 4.10: The platform with integrated battery holder exposed and the fully-assembled platform.

\subsection{Summary}

Mechanical and electrical assembly methods have be described for two different platform connector implementations. The Hook-Hole connector implementation is much easier as the platform segment pieces literally snap together and hold their form without the need for any adhesives. The drawback is the platform needs to be mechanically assembled in a pre-determined way based on the orientation of the Hooks-Holes on the individual platform segments. However, the platform segments with the interdigitated 
teeth can be assembled in any combination but additional assembly steps are required to hold the segment pieces together.

All the electrical connections for both platform types are identical. A high temperature liquidus solder paste is used to attach the ICs and passive components to the platform segment pieces. Later in the assembly process, lower liquidus temperature preformed solder spheres are used to make the orthogonal solder joints between segment pieces. This is accomplished without affecting the integrity of the existing solder connections with the electrical components. Table 4.2 summarizes the materials and equipment used for both platform connector types.

\begin{tabular}{|r|c|c|}
\cline { 2 - 3 } \multicolumn{1}{c|}{} & Hook-Hole & Teeth \\
\hline IC Solder Process & & \\
\hline Solder composition & $60 \% \mathrm{~Pb}-40 \% \mathrm{In}$ & $60 \% \mathrm{~Pb}-40 \%$ In \\
\hline Solder type & Paste & Paste \\
\hline Reflow method & Hotplate & Hotplate \\
\hline Temperature $\left({ }^{\circ} \mathrm{C}\right)$ & $230^{\circ} \mathrm{C}$ & $230^{\circ} \mathrm{C}$ \\
\hline Orthogonal Solder Joints & & \\
\hline Solder composition & $63 \%$ Sn-37\%Pb & $63 \%$ Sn-37\%Pb \\
\hline Solder type & Sphere (15 mil) & Sphere (15 mil) \\
\hline Reflow method & Hotplate & Hotplate \\
\hline Temperature $\left({ }^{\circ} \mathrm{C}\right)$ & $190^{\circ} \mathrm{C}$ & $190^{\circ} \mathrm{C}$ \\
\hline Assembly jig & Yes (FR-4) & Yes (FR-4) \\
\hline Adhesive & N/A & Silastic ${ }^{\mathrm{TM}}$ \\
\hline Heat source & N/A & Infra-red heater \\
\hline Adhesive cure recipe & N/A & $100^{\circ} \mathrm{C}$ for 15 min \\
\hline
\end{tabular}

Table 4.2: Summary of materials and equipment used to assemble both platform connection types. 


\section{Chapter 5}

\section{Inertial Measurement Unit (IMU) System Development for Using Platform}

This chapter describes the development effort to populate the platform technology with devices that can measure, store and retrieve sensor data for potential, real-world applications. For simplicity, an inertial measurement unit (IMU) system is chosen for demonstration of platform functionality. The microsystem devices can be encapsulated inside the platform since accelerometer and gyroscope data can be gathered without direct contact to the external environment. First, the hardware development is discussed including component selection criteria and the different Third-Party and custom mesoscale development boards. Next, the microcontroller software development is described which enables the sensor data logging functionality and the ability to retrieve the data from the platform and save it to a laptop for further processing. Finally, a meso-scale platform is demonstrated to verify the microcontroller programming and system functionality.

\subsection{Hardware Development}

\subsubsection{Component Selection Criteria}

Miniaturizing the overall system size is critical in order to minimize the disturbances caused to the immediate environment wherein the sensing system is to operate. This miniaturization is best accomplished by utilizing electronic and sensing 
devices that provide maximum functionality in a small footprint and which require a limited amount of external passive devices. In addition, low power is another critical system parameter as battery size, generally, plays a dominant role in determining the overall size and operational duration of a microsensing system.

The Texas Instruments MSP430 microcontroller device family is selected as the system's control and processing unit based on its rich feature set of on-chip hardware functionality, peripherals with dedicated hardware to implement standard interface protocols (SPI \& I2C), small packaging form factor options and various low-power modes with ultra-fast wake-up times of less than $1 \mu$ s from standby mode. In addition, there is an abundance of application literature and hardware/software development platforms available from both Texas Instruments and Third-Party organizations.

The ES2274 Development Board by SoftBaugh, Inc. is used as the hardware development platform. The ES2274 board, depicted in Fig. 5.1 provides access to all the on-board MSP430F2274 ports and peripherals in addition to external Flash memory, a connector for $\mathrm{I} 2 \mathrm{C}$ communications and an RS-232 driver and connector to interface to a PC for external data storage. Therefore, the entire system functionality can be verified prior to implementing it on the miniaturized silicon platform. 


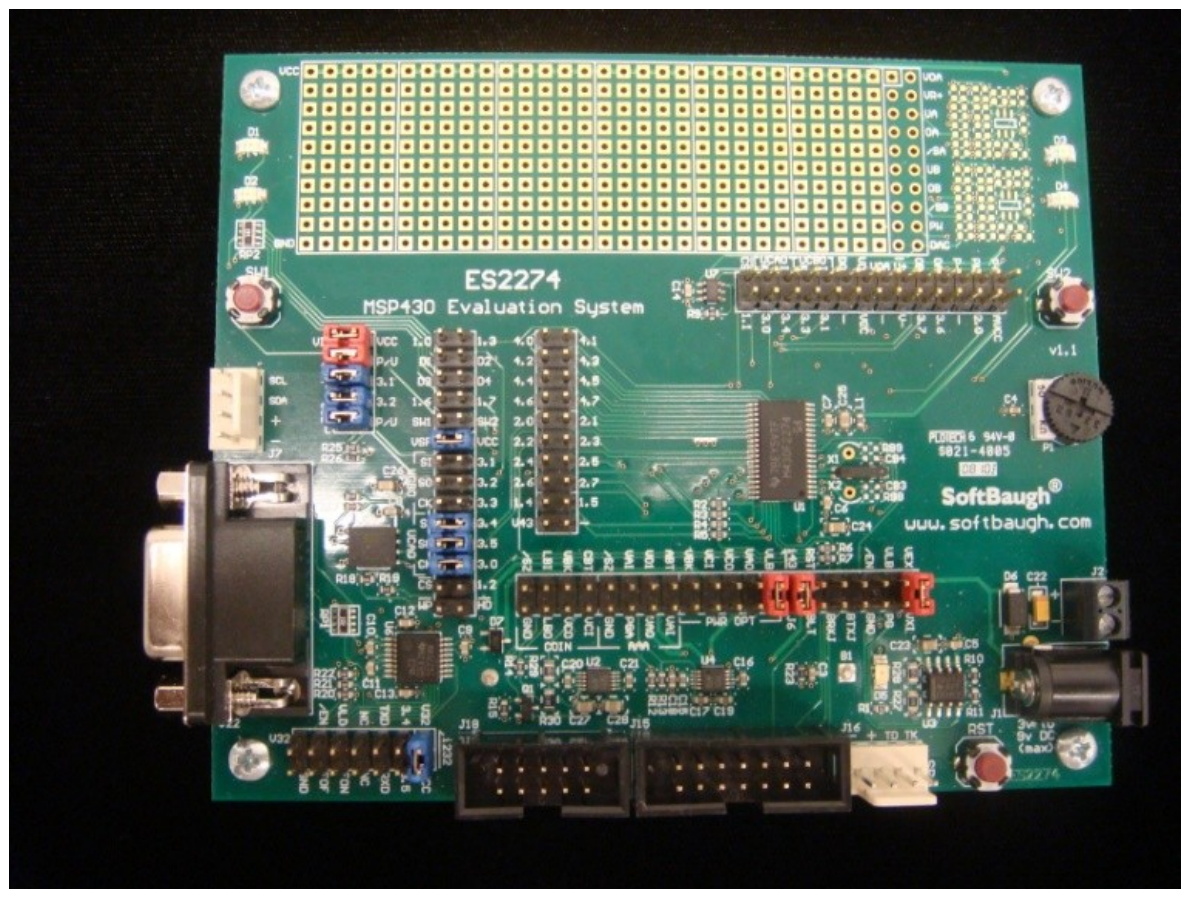

Figure 5.1: SoftBaugh, Inc. ES2274 development board.

As mentioned above, reducing the package footprint is essential to create a miniaturized sensing platform. In addition, limiting the number of external passive components that occupy a large area is also critical in miniaturization of the system. Based on these two design parameters, system components were selected that have serial, digital communication functionality to reduce package pin counts and that also had integrated analog functionality to reduce the number of external passive devices and overall power consumption.

For instance, the ES2274 board has an external SST25VF020QA, 2M-bit Flash memory device, by Silicon Storage Technology that interfaces directly to the MSP430F2274 via a serial peripheral interface (SPI). The device has an 8 -contact (5 mm x $6 \mathrm{~mm}$ x $0.8 \mathrm{~mm}$ ) package and consumes $7 \mathrm{~mA}$ (typical active read current) and $8 \mu \mathrm{A}$ of typical standby current. Based on the microsystem's sample frequency of $100 \mathrm{~Hz}$, this 
device is able to hold $\sim 3.5$ minutes of six degrees-of-freedom acceleration and gyroscope platform data.

The accelerometer and gyroscope selection began with searching for commercial, off-the-shelf devices with these two attributes in addition to the sensors' range and sensitivity. The selected accelerometer, BMA020, is a digital, triaxial acceleration sensor by Bosch Sensortec. It is housed in a 12-contact ( $3 \mathrm{~mm} \times 3 \mathrm{~mm} \times 0.9 \mathrm{~mm}$ ) package and requires only two external decoupling capacitors for its analog and digital power supplies. Under typical operating conditions and stand-by mode, the device consumes $200 \mu \mathrm{A}$ and $1 \mu \mathrm{A}$, respectively, from a 3.3V power supply. In addition, the device can support both SPI and Inter-integrated Circuit Bus (I2C) communication protocols to transmit the triaxial acceleration data. The $\mathrm{I} 2 \mathrm{C}$ interface is utilized herein and will be discussed later in this chapter.

The gyroscope that was selected is the ITG-3200 digital output, 3-axis MEMS gyroscope by InvenSense. It is packaged in a 24-contact ( $4 \mathrm{~mm} \times 4 \mathrm{~mm} \times 0.9 \mathrm{~mm}) \mathrm{QFN}$ package. However, only 10 contacts are actually used for device operation. Under typical operating conditions and stand-by mode, the device consumes $6.5 \mathrm{~mA}$ and $5 \mu \mathrm{A}$ of current, respectively, from a $3.3 \mathrm{~V}$ power supply. The device requires four external capacitors for decoupling, voltage regulator filtering and charge pump functionality. The device's only external communication interface uses the I2C protocol.

Since $\mathrm{I} 2 \mathrm{C}$ is a bus protocol, numerous slave devices can share two wires and perform bi-directional communication with a master device. In the platform design, the accelerometer and the gyroscope share the I2C bus and are placed in slave mode. The 
MSP430 microcontroller is placed in master mode and controls all communication and data transmission on the $\mathrm{I} 2 \mathrm{C}$ bus. Therefore, besides power and ground, only two additional traces are required to facilitate bi-directional communication between the sensors and the microcontroller, which significantly reduces the amount of connections needed for system realization. The only drawback is the $\mathrm{I} 2 \mathrm{C}$ communication requires an external pull-up resistor for each bus wire, placed close to the microcontroller pins. However, this is a minor penalty compared to other communication protocols and especially sensors with analog outputs which typically require a dedicated signal for each sensing axis in addition to all the external passive components required.

\subsubsection{Microcontroller to Sensor Interface Development Board}

A two-layer PCB board is designed to hold the BMA020 accelerometer and ITG3200 gyroscope, and the six additional external passive capacitors. The board was manufactured using ExpressPCB's MiniBoardPro manufacturing process as shown in Fig. 5.2. Both sensors share a common I2C bus consisting of two signals, namely SDA and SCL, serial data and serial clock, respectively. Power (3.3V) and ground signals, provided by the SoftBaugh Development Board, are the only additional connections needed to successfully provide bidirectional communications from the microcontroller to both sensors. These I2C interface connections are shown in Fig. 5.3. 


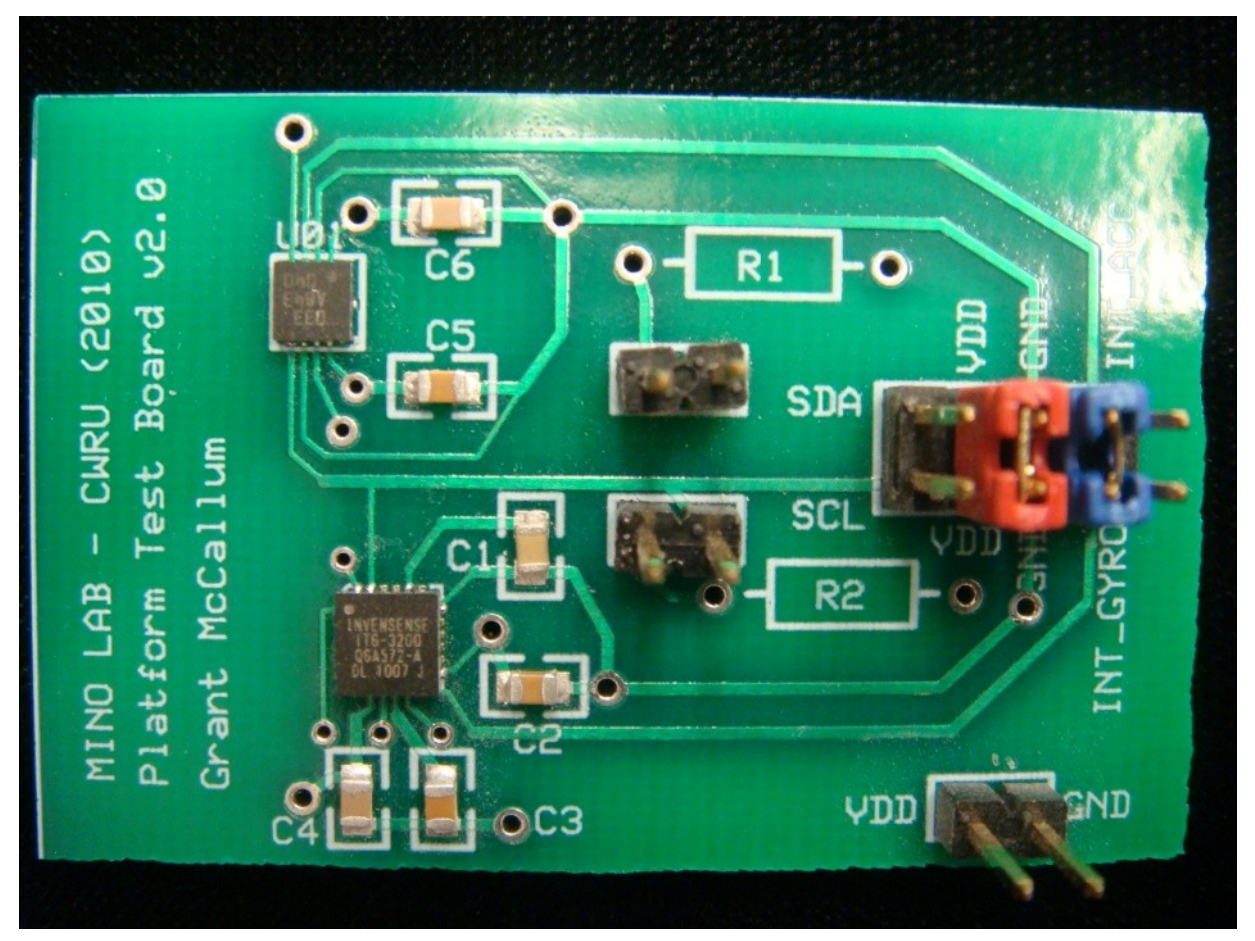

Figure 5.2: Custom sensor development board.

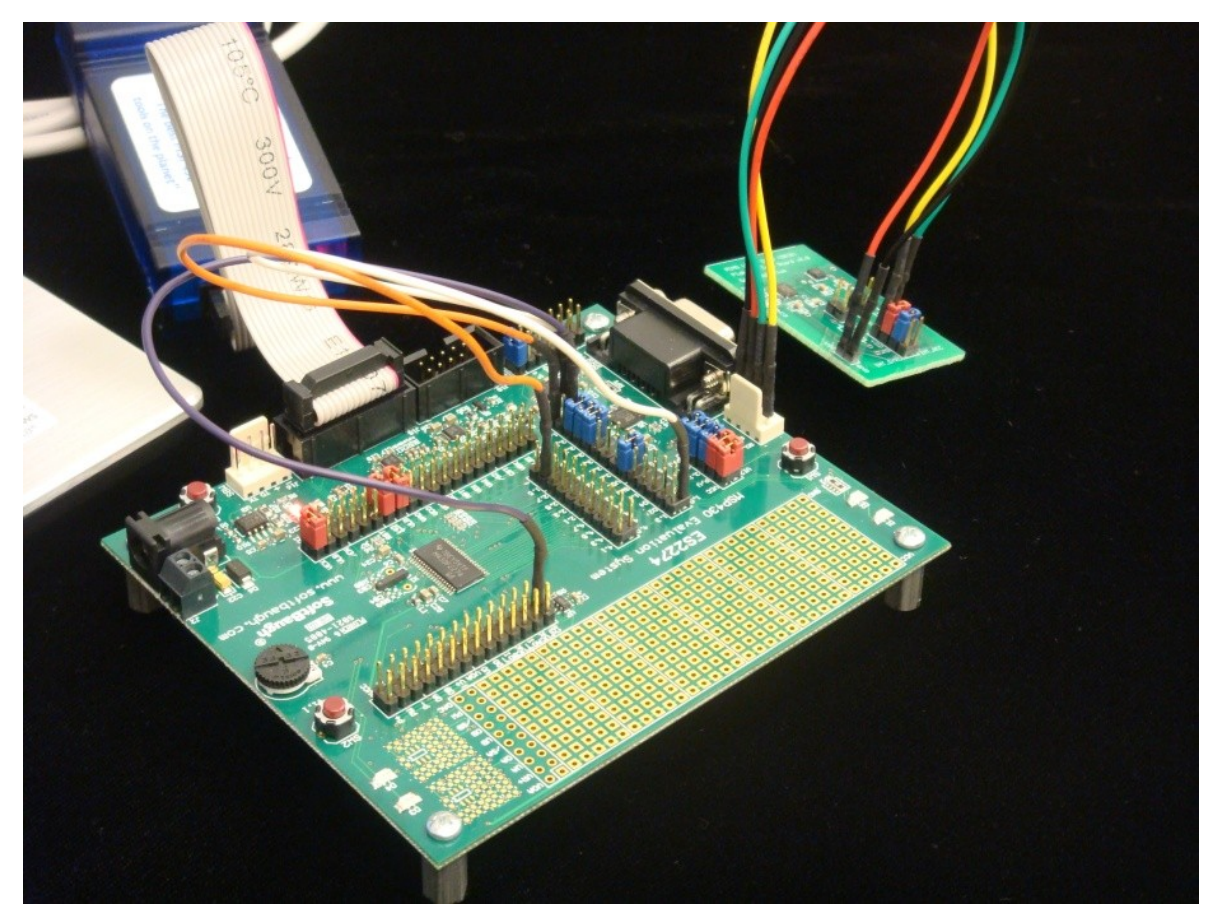

Figure 5.3: $\mathrm{I} 2 \mathrm{C}$ connections to sensor development board. 


\subsubsection{Hardware for Transferring Data to External Personal Computer (PC)}

Once the platform has gathered all the sensor data for a particular application, the data needs to be transmitted out of the platform's Flash memory device and saved to an external PC for further processing. Asynchronous communications via an RS-232 connection can achieve this with three wire connections (Power, Ground and TX) from the platform to an external printed circuit board that contains an RS-232 transceiver and connector. The Maxim MAX3232E True RS-232 Transceiver was used to interface between the MSP430 microcontroller and a PC serial port as shown in Fig. 5.4.

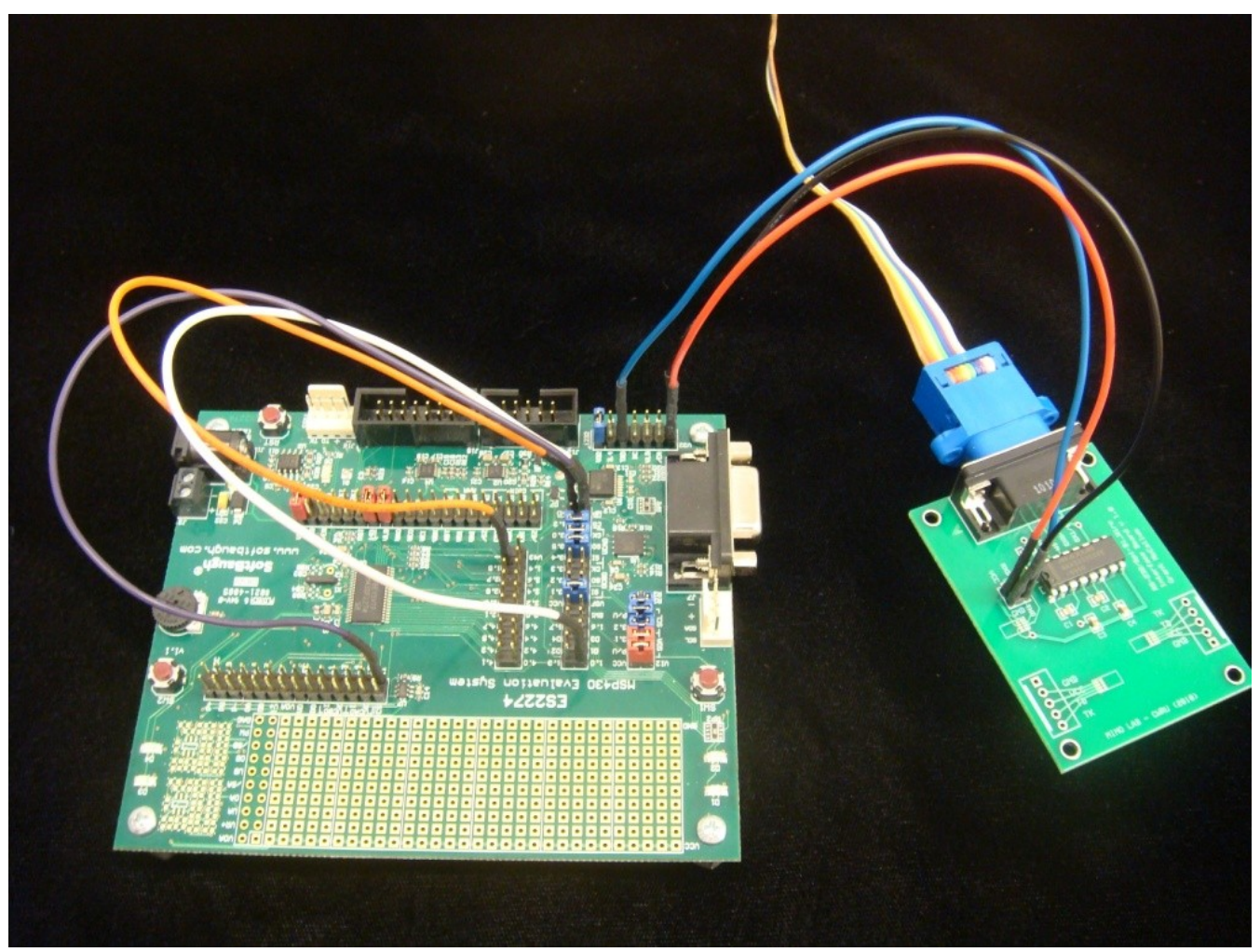

Figure 5.4: RS-232 development board connections. 
The MSP430 microcontroller's internal Timer hardware can be used to create a software-based Universal Asynchronous Receive Transmit (UART) function to implement the RS-232 protocol [71]. This feature is used because the MSP430 UART peripheral needed to be configured as a SPI interface in order to retrieve the data from the external Flash memory device. The output signal of the Timer hardware can be controlled and directed to an MSP430 output pin which becomes the transmit signal (TX) from the platform to the RS-232 transceiver. The RS-232 implemented connection is a 9600 baud, with 8 bits of data, no parity bit and one stop bit or, in other words, an 8-N-1 format for each frame.

\subsection{Software Development}

The following section describes the software development for the Texas Instruments MSP430 microcontroller to provide the platform's data-logging functionality and the ability to retrieve the sensor data and store it to a personal computer. All software is written in the C computing language and compiled using IAR Systems' Embedded Workbench development environment. The SoftBaugh programmer/emulation tool, USBP Pro, was used to download the compiled code to the MSP430 and also for

emulation debug of the system. The complete software development environment is shown in Fig. 5.5 


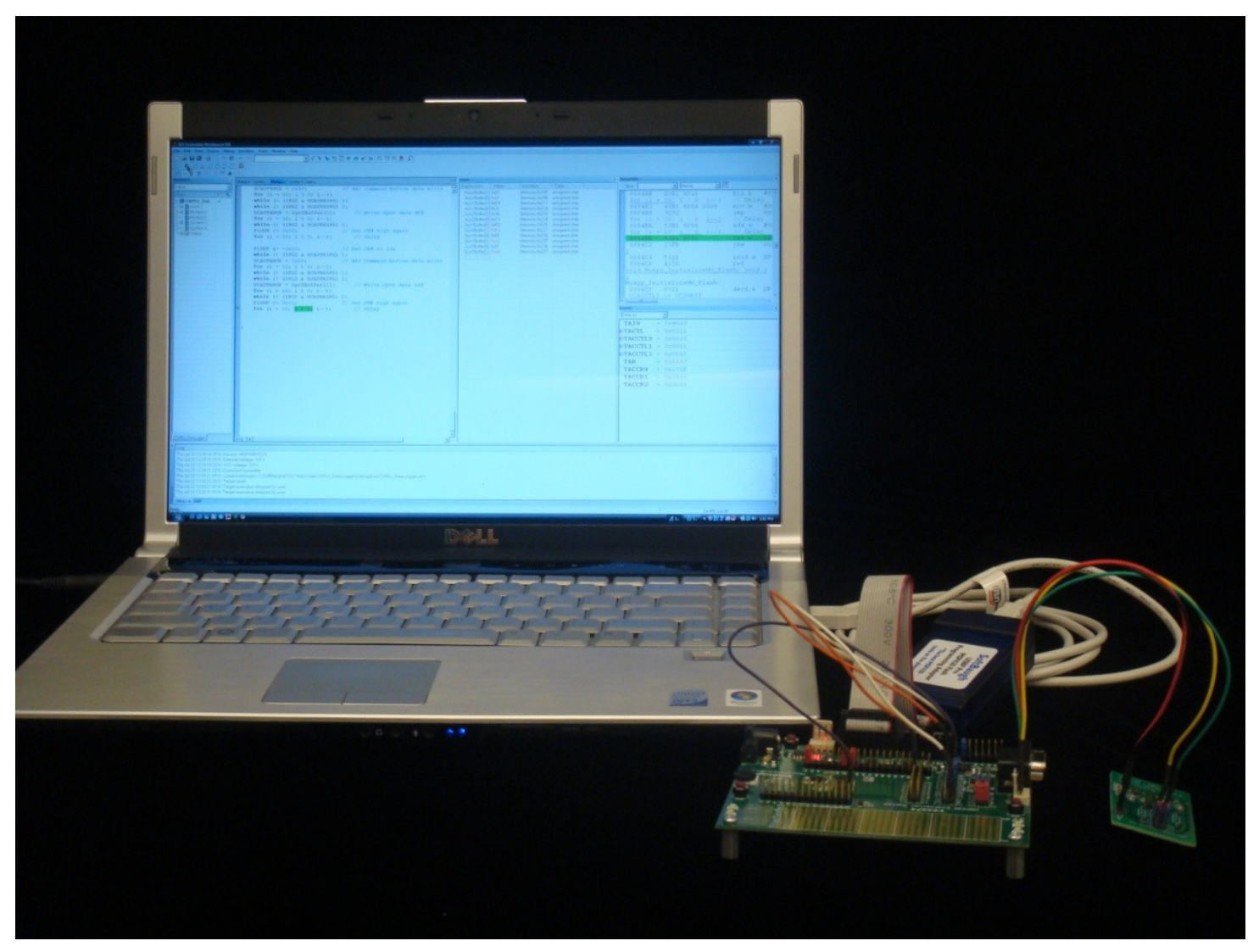

Figure 5.5: Complete software development system.

\subsubsection{Software for Data-logging Functionality}

As a general overview, the data-logging function acquires the three-axis acceleration data and three-axis gyroscope data at a rate of $100 \mathrm{~Hz}$. For each sample period the data is stored in the MSP430 internal SRAM and then transferred and stored in an external Flash memory. The data-logging function can be broken into a well-defined flow as shown in Fig. 5.6. The actual C language code is presented in Appendix B. 


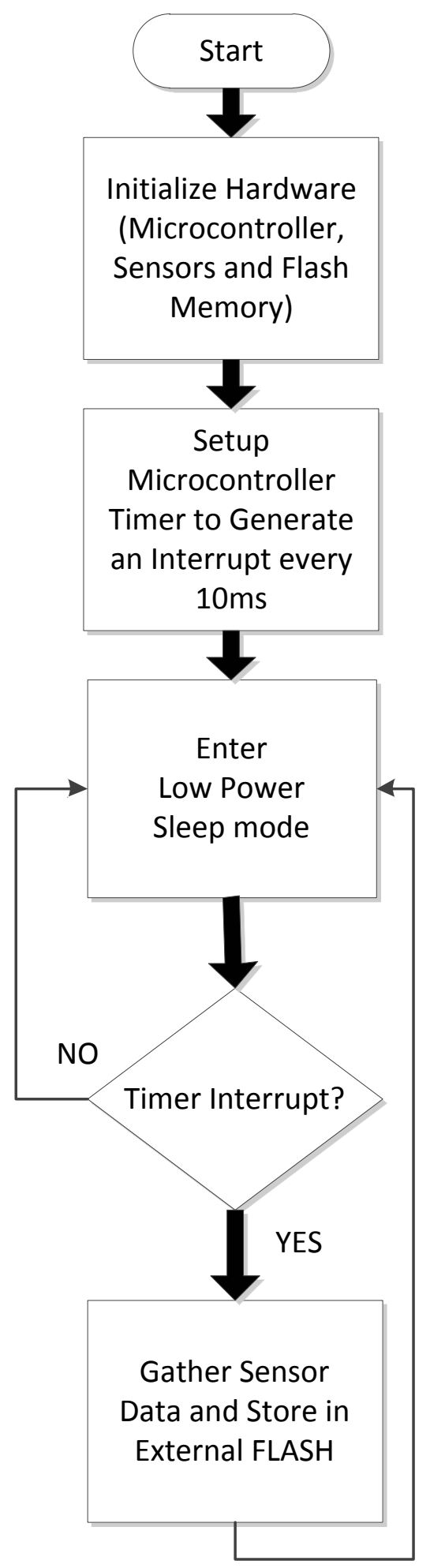

Figure 5.6: Microcontroller data-logging flowchart. 
The software complexity is greatly reduced by using many of the dedicated hardware peripherals of the MSP430 microcontroller that simplify external communication to other devices. Two of these dedicated interface protocols namely, Serial Peripheral Interface (SPI) and Inter-integration Circuit Bus (I2C), require some initialization as described below.

The system hardware initialization entails selecting the MSP430 digitally controlled oscillator (DCO) to generate a $1 \mathrm{MHz}$ core clock which is, or a divided form of it is, used in all the microcontroller's peripheral and internal functional blocks. Next, two ports of the MSP430 microcontroller need to be configured which are Port_1 for the external Flash memory control signals and Port_3 for both the SPI interface to the external Flash memory, and the IC2 interface to the accelerometer and gyroscope sensors.

With the SPI and I2C interfaces now configured, the external Flash memory and the gyroscope devices need to be configured before the data-logging routine can begin. The external Flash memory configuration is performed via the SPI interface to the MSP430 microcontroller and involves the following procedure: First a write command is issued to the memory to set the device's status register to write mode and enable the Automatic Address Increment (AAI) feature of the Flash memory device. Next, a writeenable command is issued and then a chip-erase command to set all the data locations to the value $0 x F F h$. Then another write-enable command is issued and then the AAI initial address location is sent to the Flash memory to indicate where it should begin storing the data. After each successful write operation to the Flash memory, the device automatically advances the internal pointer to the next memory location. 
The gyroscope initialization is performed via communication from the microcontroller using the $\mathrm{I} 2 \mathrm{C}$ communication bus. Since both the accelerometer and the gyroscope share the same communication bus, the first step is to send the gyroscope device address on the bus to alert it that more instructions are forthcoming. The first initialization step is to configure the Sample Rate Divider register to set how often new gyroscope data will be written to the gyroscope's data registers to be read. This is configured for the platform to be every $8 \mathrm{msec}$. Next, the Digital Low-Pass Filter and Full-Scale register are configured so the internal sampling rate is $1 \mathrm{kHz}$ and the internal low-pass filter bandwidth is $188 \mathrm{~Hz}$. Also, the full-scale of the gyroscope is configured to $\pm 2000 \%$ sec. The final configuration is the gyroscope's Power Management register; it is set in a way that the gyroscope uses an internal phase-locked loop (PLL) with an x-axis reference, due to improved stability as noted by the gyroscope manufacturer.

An internal MSP430 Timer is used to generate an interrupt every $10 \mathrm{msec}$ $(100 \mathrm{~Hz})$ to notify the microcontroller to gather and store the available sensor data. Completing the hardware initialization process, the final step is to configure the MSP430 Timer to count from zero to 9,999 (i.e., $10,000 \times$ core clock period $=10 \mathrm{~ms}$ ), issue a system interrupt, and then repeat. The local Timer interrupt is enabled just prior to entering the main program loop which completes the hardware initialization step.

The main program contains the remaining two major sections for the data-logging function which is contained in an endless while loop. First the MSP430 is placed in lowpower mode zero. Per the above discussion, every $10 \mathrm{~ms}$ the Timer issues an interrupt and the MSP430 wakes up from the low-power mode and performs the following 
functions: Reads the data from the sensors and stores the sensor data to the external Flash memory.

The process to read the data from the sensors is as follows: (i) configure the MSP430 I2C peripheral to operate as a master and set the bus clock frequency to 100 $\mathrm{kHz}$; (ii) transmit the accelerometer device address to grant access to the bus; and (iii) transmit the command to read the sensor data. Once this is completed, the bus is stalled and the MSP430 I2C is configured for receive mode. The I2C protocol allows for one byte ( 8 bits) of data to be transmitted in a frame. The Bosch BMA020 accelerometer produces 10-bits of data for each axis. The first 8 bits of the acceleration data contains the lowest significant bits $\langle 1: 0\rangle, 5$ bits of unused data and the final bit is a control bit indicating if new data is present in the register. The next 8 bits that are transmitted contain acceleration data for the most significant bits $<9: 2>$. Therefore, it takes two read operations to extract the acceleration data for each axis. Each of the six read operations then stores 8 bits of acceleration data to an internal MSP430 SRAM location resulting in six memory locations being utilized to temporary store all the acceleration data for the sample period. The order of acceleration data read by the MSP430 microcontroller is as follows: $\mathrm{X}$-axis bits $<1: 0>, \mathrm{X}$-axis bits $<9: 2>$, Y-axis bits $<1: 0>$, $\mathrm{Y}$-axis bits $<9: 2>$, Zaxis bits $<1: 0>$, Z-axis bits $<9: 2>$. The data is presented in 2's compliment format with a range for the $2 \mathrm{~g}$ configuration of $-2.000 \mathrm{~g}$ to $+1.996 \mathrm{~g}$ in increments of $\pm 0.004 \mathrm{~g}$.

After all the acceleration data has been stored to the MSP430 internal SRAM, the I2C interface is placed in reset and reconfigured for MSP430 transmit mode. The gyroscope's address is transmitted on the $\mathrm{I} 2 \mathrm{C}$ bus to grant the gyroscope access to the bus. Next, the initial gyroscope register location to be read is transmitted. The I2C 
interface is again placed in reset and reconfigured for the MSP430 receive mode to accept the gyroscope sensor's data. The InvenSense ITG-3200 gyroscope produces two bytes (16 bits) of data for each axis. So, similar to the Bosch BMA020 device, two read operations are required to gather the complete sensor data for each device axis. As before, each of the six bytes of data is stored in a temporary MSP430 internal SRAM location. The gyroscope data is also 2 's compliment format with a range of $+2000^{\circ} / \mathrm{sec}$ to $-1999.939^{\circ} / \mathrm{sec}$ with increments of $\pm 0.061^{\circ} / \mathrm{sec}$. The order in which the gyroscope data is read from the sensor is as follows: $\mathrm{X}$-axis bits $<15: 9>$, $\mathrm{X}$-axis bits $<8$ :0 $>$, $\mathrm{Y}$-axis bits $<15: 9>$, $\mathrm{Y}$-axis bits $<8: 0>, \mathrm{Z}$-axis bits $<15: 9>$ and Z-axis bits $<8: 0>$.

At this point of code execution, all 12 bytes of the acceleration and gyroscope data resides in internal MSP430 SRAM memory locations. The final step of the datalogging function is to transfer all this data to the external Flash memory from the microcontroller via the SPI interface. Similar to the I2C protocol, the SPI protocol transmits frames in bytes $(8$ bits). Transmitting the data to the external Flash memory begins with an AAI (Automatic Address Increment) command transmitted to the Flash and then an immediate transmission of the 8 bits of data to be stored in the Flash. This process is repeated until all desired data is completely transmitted. The order the data is stored to the external Flash is as follows: $\mathrm{X}$-axis acceleration least significant bits (LSB), $\mathrm{X}$-axis acceleration most significant bits (MSB), Y-axis acceleration LSB, Y-axis acceleration MSB, Z-axis acceleration LSB, Z-axis acceleration MSB, X-axis gyroscope LSB, X-axis gyroscope MSB, Y-axis gyroscope LSB, Y-axis gyroscope MSB, Z-axis gyroscope LSB and, Z-axis gyroscope MSB. 
Once the final sensor data writing process to external Flash memory completes, the microcontroller is placed back into low-power mode zero and waits for another Timer interrupt to repeat the process described above of reading the sensor data and storing it to the external Flash. The data-logging code takes up $2.768 \mathrm{~KB}$ of MSP430 program memory and takes 6,813 total clock cycles to gather all the sensor data and save it to the external Flash memory. With a 2Mbit external Flash memory device, there are 262,140 memory locations and with 12 bytes of sensor data gathered per sample period, it results in 21,845 samples to fill up the 2 Mbit Flash device. With a sample rate of $100 \mathrm{~Hz}$, this will be equivalent to $\sim 3.64$ minutes of data-logging operation capacity.

\subsubsection{Software to Retrieve Platform Data and Store on a PC}

As mentioned above, the IAR Systems' Embedded Workshop compiler/emulation tool was used in conjunction with the SoftBaugh ES2274 development board and a custom RS-232 transceiver board to design and test the software, which enables the MSP430 to transfer the logged data from the external Flash memory to an external personal computer for further processing. The following describes the $\mathrm{C}$ language software program that is downloaded to the MSP430 microcontroller to perform this data retrieval function.

Figure 5.7 is a flow diagram that shows the software functionality for retrieving the platform sensor data. First, the MSP430 microcontroller and external Flash memory device need to be configured. As before, the microcontroller's digital controlled oscillator (DCO) is configured to create a $1 \mathrm{MHz}$ core clock. Two microcontroller ports are then configured. Port_1 creates the three external Flash memory control signals for the 
communication interface as described above: write-protect, memory enable and hold. Also, one of the Port_1 pins is defined as an output to supply the RS-232 TX signal to the personal computer. Port_3 is configured to enable the SPI interface signals, SCLK, SIMO (slave-in, master-out) and SOMI (slave-out, master-in) from the MSP430 to the external Flash memory device. The C-language program that was developed to enable platform data transmission to an external PC via an RS-232 connection can be found in Appendix C.

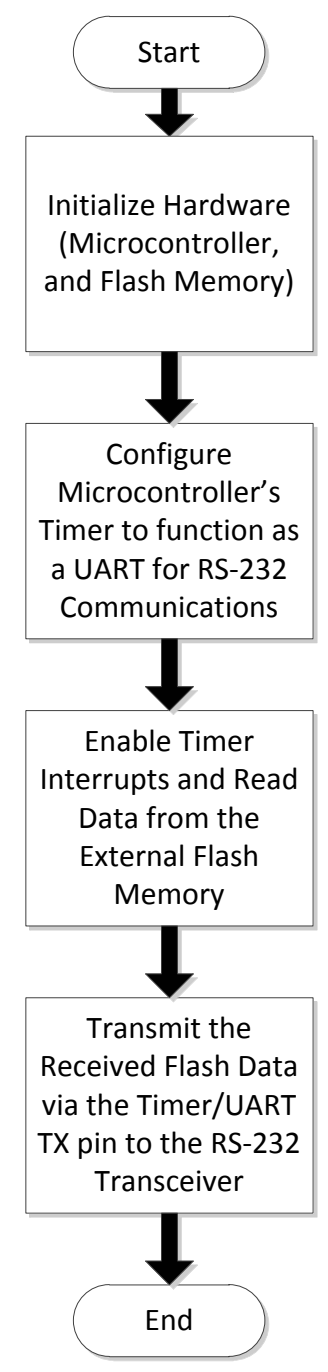

Figure 5.7: Sensor data retrieval via an RS-232 connection. 
Similar to the data-logging software, the external Flash memory needs to be initialized by setting the SPI clock to a value of $100 \mathrm{kHz}$ for interface communications and setting the MSP430 microcontroller as the master.

As previously mentioned, the MSP430 Timer can be used to create software UART for asynchronous communications to a personal computer. Since we are only interested in transmitting data from the platform, the receive functionality of the Timer UART is not implemented. The next step in the software code is to configure the MSP430 Timer for UART functionality. This is accomplished by configuring two control registers to use the $1 \mathrm{MHz}$ core clock as the Timer clock and run the Timer in continuous mode so that is counts from zero to $0 \mathrm{xFFFFh}$ and then repeats. Also, to select the Timer's output to be controlled by the value of the register OUT. The Timer OUT signal will become the RS-232 transmit signal, TX, as discussed above. Finally, a register is set to act as a TX flag to indicate when the Timer UART is ready to accept new data for transmission. A Timer interrupt service routine (ISR) will be used to control the timing of the Timer UART data transmission.

Next, the MSP430 microcontroller global interrupts are enabled which enables the Timer interrupts described above. The final step is to issue a read command from the MSP430 to the external Flash memory controller and the beginning read address location. The Flash memory will then send a byte of data to the microcontroller. Once a byte is received by the microcontroller, the data is written to a register and a Timer interrupt is forced to begin the data transmission from the Timer UART to the RS-232 transceiver and ultimately to be stored on the personal computer. This process is automatically repeated until all the external Flash memory locations have been read. If the read 
operation reaches the last Flash memory location in the device, the next read will start at address location zero and continue. During the Timer ISR, the data is shifted one bit at a time and held for a predetermined duration (BITTIME) which is dependent on the baud rate before shifting in a new bit value for the TX/OUT signal, defined by equation 5.1 [71].

BITTIME $=($ Clock Frequency $[\mathrm{Hz}]+$ Baud Rate/2 $) /($ Baud Rate $)$

The Timer ISR also generates the required start and stop bits for the asynchronous communication.

\subsection{Integrated Meso-Scale Sensing Platform}

In an effort to prove the proposed platform system functionality, a meso-scale system was design and created. The meso-scale system is comprised of two miniature printed circuit boards: (i) the first containing the accelerometer and gyroscope sensing devices and two $1.55 \mathrm{~V}$ coin-cell batteries to power the system; and (ii) the second containing the interface to the programmer and RS-232 transceiver, the charge pump integrated circuit, MSP430 microcontroller, and external Flash memory device for datalogging. The two PCB boards stacked on top of each other via a two-pin connector for battery power and ground, and also a four-pin connector for the $\mathrm{I} 2 \mathrm{C}$ interface from the microcontroller to the sensors as seen in Fig. 5.8. 


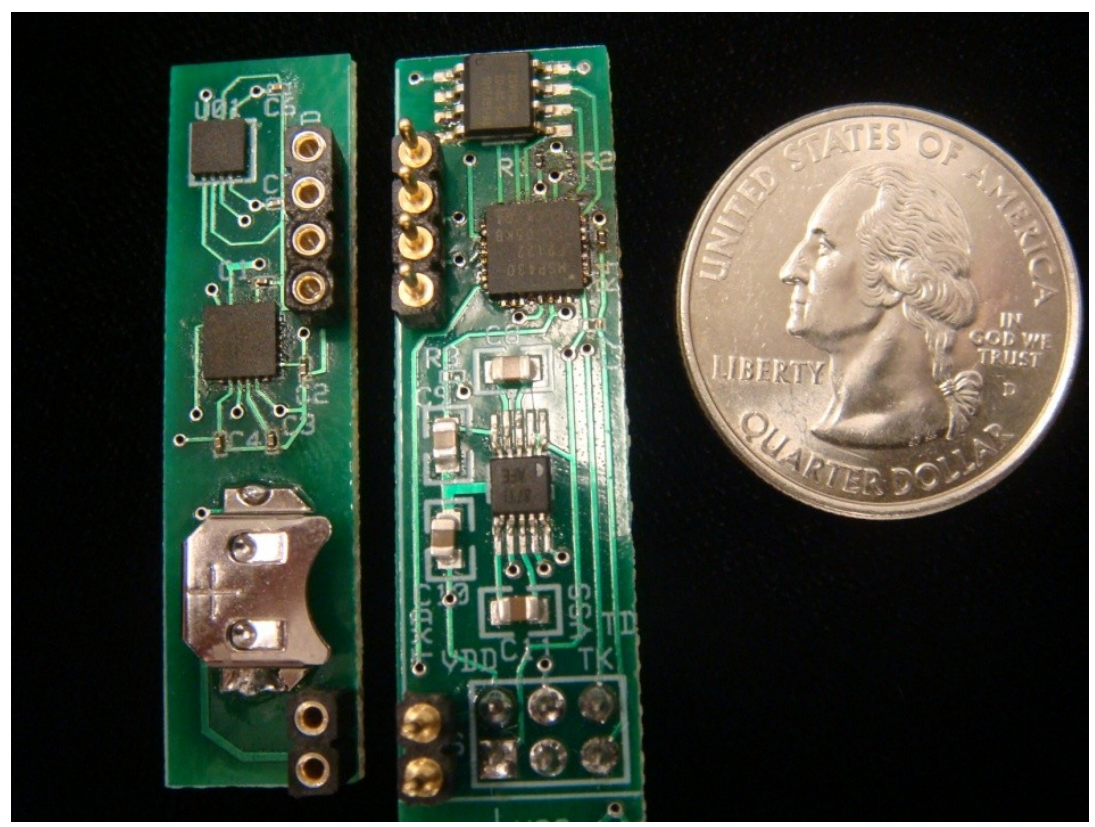

Figure 5.8: Complete meso-scale platform system.

The purpose of the meso-scale system is: (1) to eliminate the use of the SoftBaugh, Inc. ES2274 Development Board and prove the microcontroller can be independently programmed via the two-pin, Spy-Bi-Wire interface; (2) to create and verify an autonomous battery operated system solution that properly collects the sensor data; and (3) to use the existing MSP430 microcontroller code with minor code changes to use a lower pin count and lower cost MSP430F2122 device rather than the larger MSP430F2274 device on the ES2274 Development Board.

\subsubsection{Meso-scale Platform: System Schematic and Board Design}

Figure 5.9 is the system schematic of the complete meso-scale platform. From the schematic, one can see the system is powered by two $1.55 \mathrm{~V} 377 / 376$ coin-cell batteries connected in series and providing a $3.1 \mathrm{~V}$ input to the Texas Instruments TPS60211 low-ripple charge pump. The charge pump circuitry is required to maintain a 
constant $3.3 \mathrm{~V}$ system voltage and to provide a maximum $100 \mathrm{~mA}$ drive current to the rest on the platform since the coin-cell battery's maximum current drive is $34 \mu \mathrm{A}$ at $1.53 \mathrm{~V}$ with a $45 \mathrm{k} \Omega$ load.

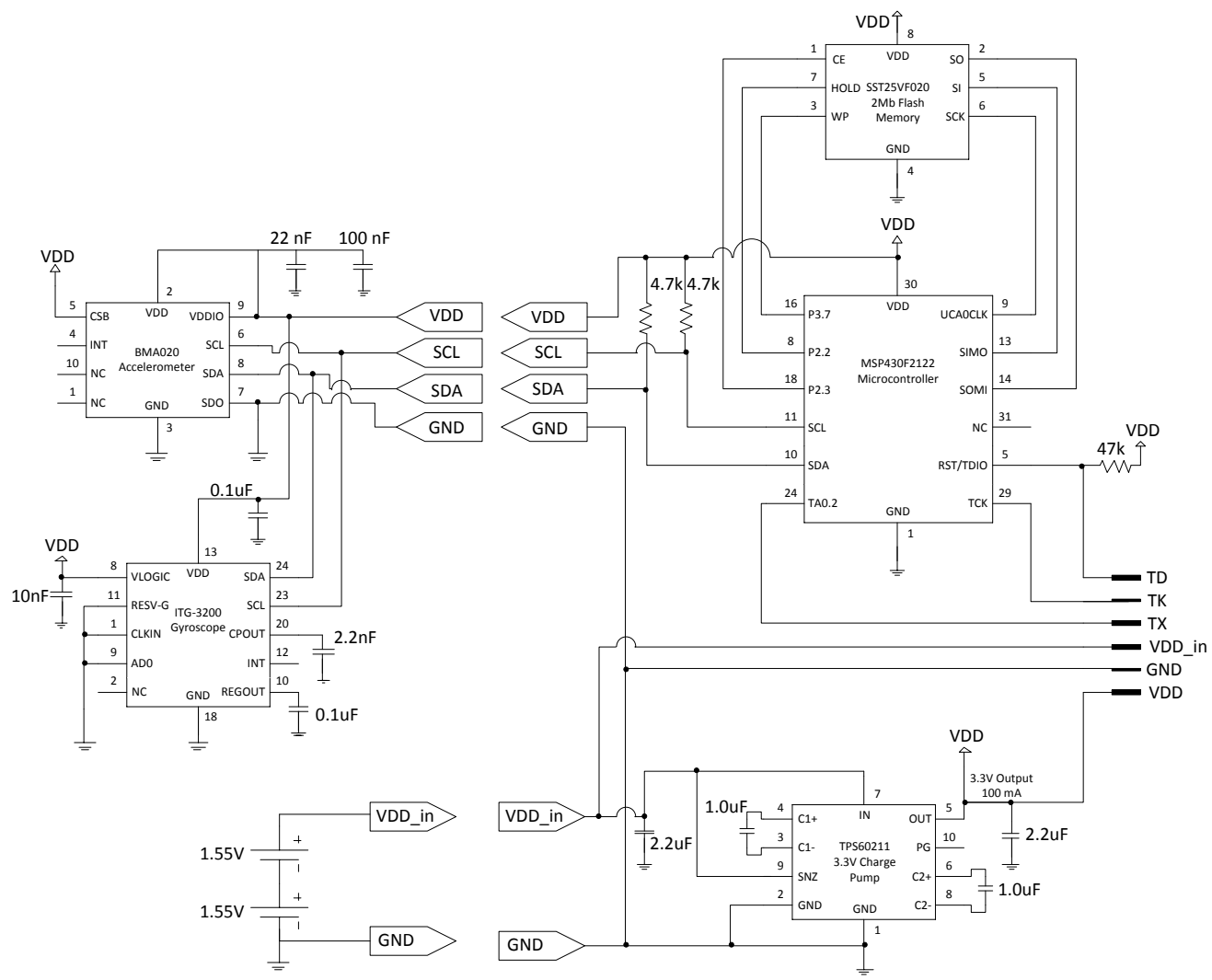

Figure 5.9: Schematic of complete meso-scale sensing platform.

The microcontroller board contains a five pin interface to provide power (VDD), ground (GND), and the Spy-Bi-Wire (TD-Data, TK-Clock) connections to the microcontroller for programming as shown in Fig. 5.10. The fifth pin is the transmit data output (TX) from the microcontroller to the RS-232 transceiver device for platform data retrieval to an external computer and is shown in Fig. 5.11. The remainder of the mesoscale platform hardware and software is identical to what was already described in Sections 5.1 and 5.2. 


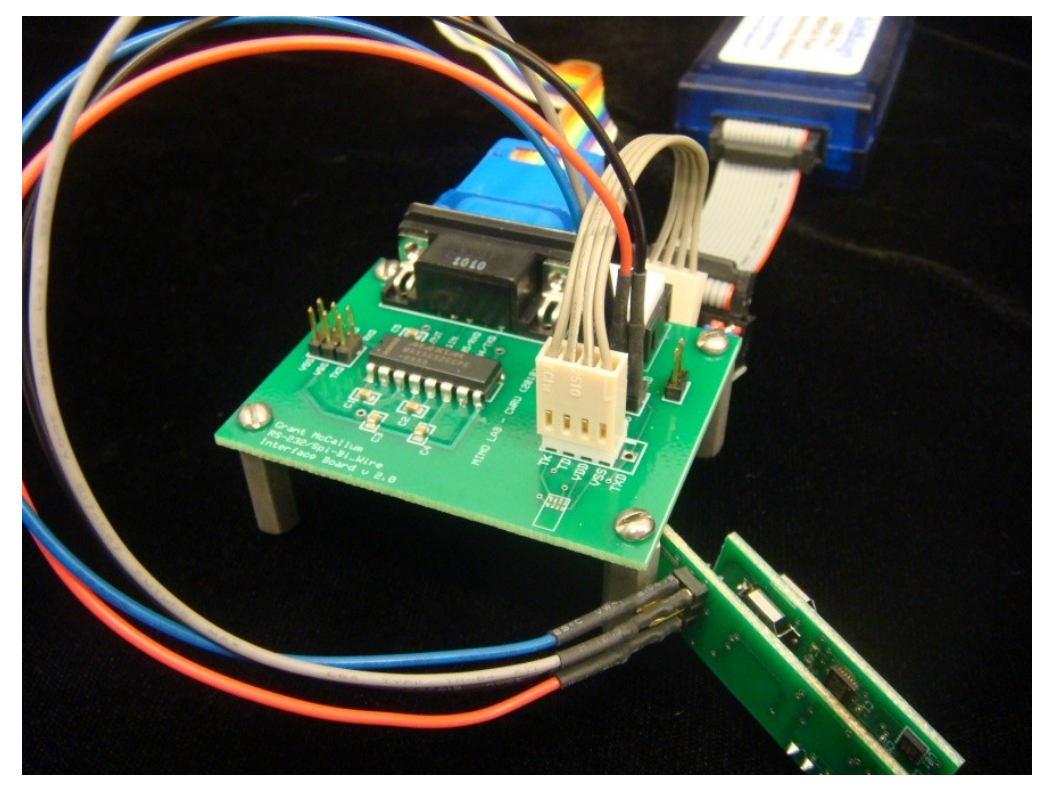

Figure 5.10: Spy-Bi-Wire connections for microcontroller programming.

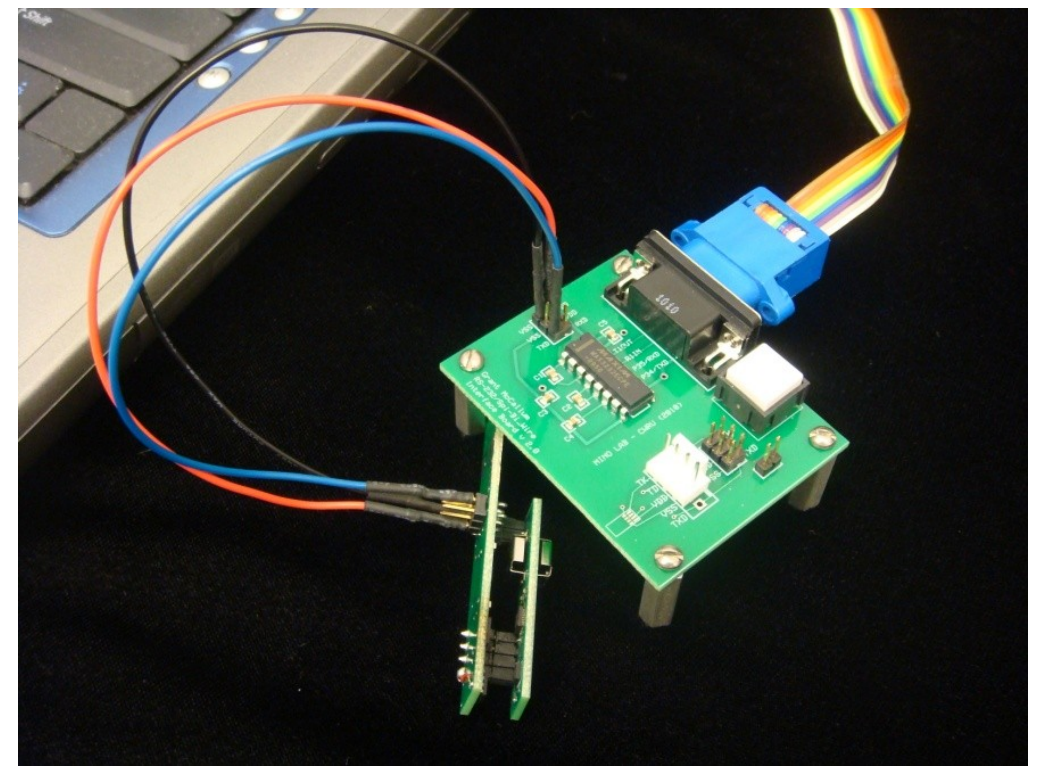

Figure 5.11: Connections for data retrieval via RS-232 interface. 


\subsection{Microsystem Platform Implementation of the IMU}

This chapter describes the effort to transfer the meso-scale IMU system described in this chapter onto the silicon platform technology discussed in Chapter 2. Therefore, the result will be a fully, autonomous IMU system housed within a platform structure that is made entirely of silicon. The platform structure will be $12 \times 12 \times 25 \mathrm{~mm}^{3}$ in dimension and use the $1 \mathrm{~mm}$ long interdigitated teeth features as the connector method. Silicon wafers of type (111) that are $250 \mu \mathrm{m} \pm 10 \mu \mathrm{m}$-thick and double-sided polished will be used in the microfabrication process.

All the system electronics, sensors, passive devices and batteries will be contained within the silicon platform structure. The only part of the system that will be exposed to the outside environment will be the eight-pin micro-connector to enable microcontroller programming and IMU data retrieval. A mechanical drawing of the proposed silicon platform structure is depicted in Fig. 5.12.

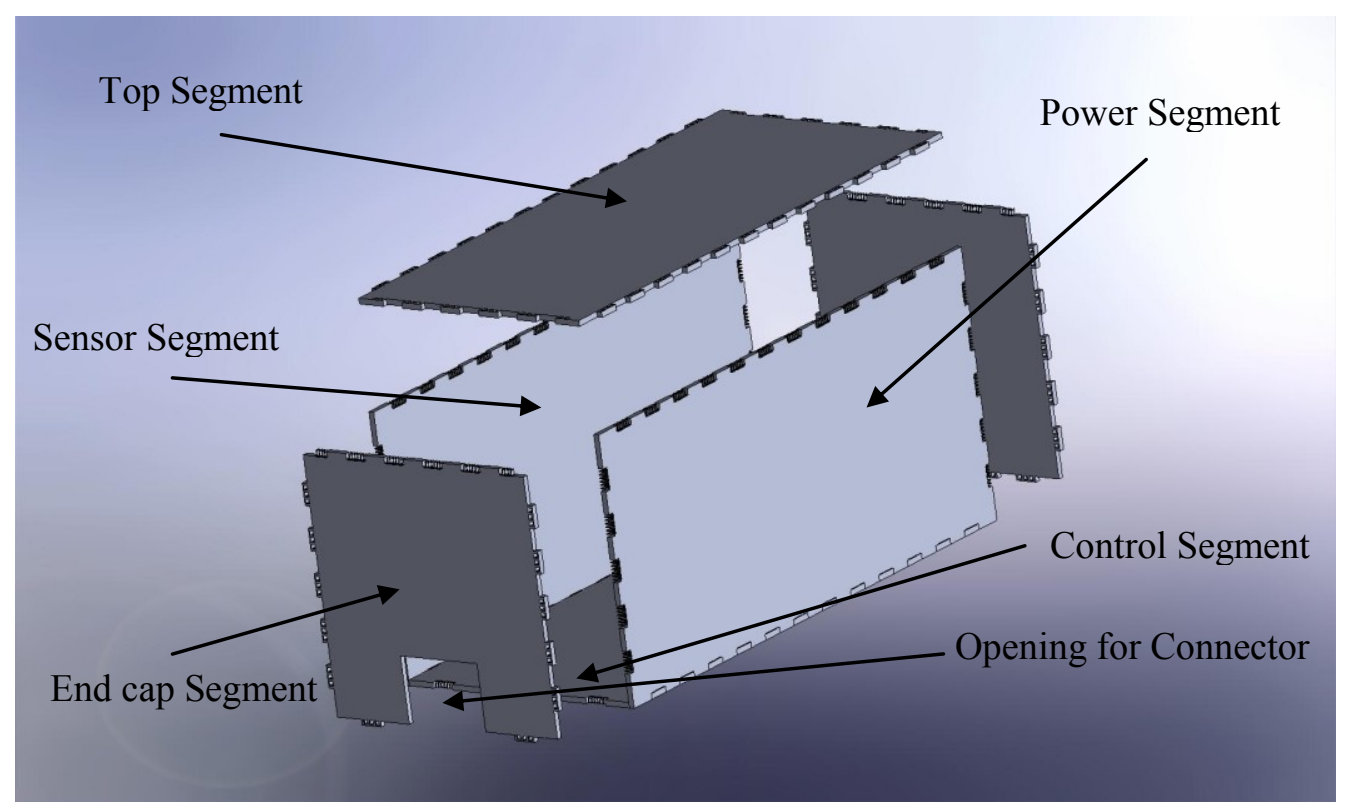

Figure 5.12: Three-dimensional view of the proposed silicon-based platform. 
The sensor segment will contain both the three-axis gyroscope and accelerometer in addition to the passive components these devices need. The control segment will contain the 8-pin external connector, microcontroller, Flash memory and the pull-up resistors needed for the $\mathrm{I} 2 \mathrm{C}$ bus and microcontroller reset signal. The power segment will contain the charge-pump IC and the necessary capacitors for proper operation.

In addition, four orthogonal solder joints will be made between the control and sensor segments to facilitate $\mathrm{I} 2 \mathrm{C}$ communication and power and ground signals. Three orthogonal solder joints will be created between the control and power segments: two signals from the battery (power-in and ground) and another from the charge-pump to the rest of the circuit (a 3.3V , high current-drive power supply). The complete twodimensional version of the platform is shown in Figure 5.13.

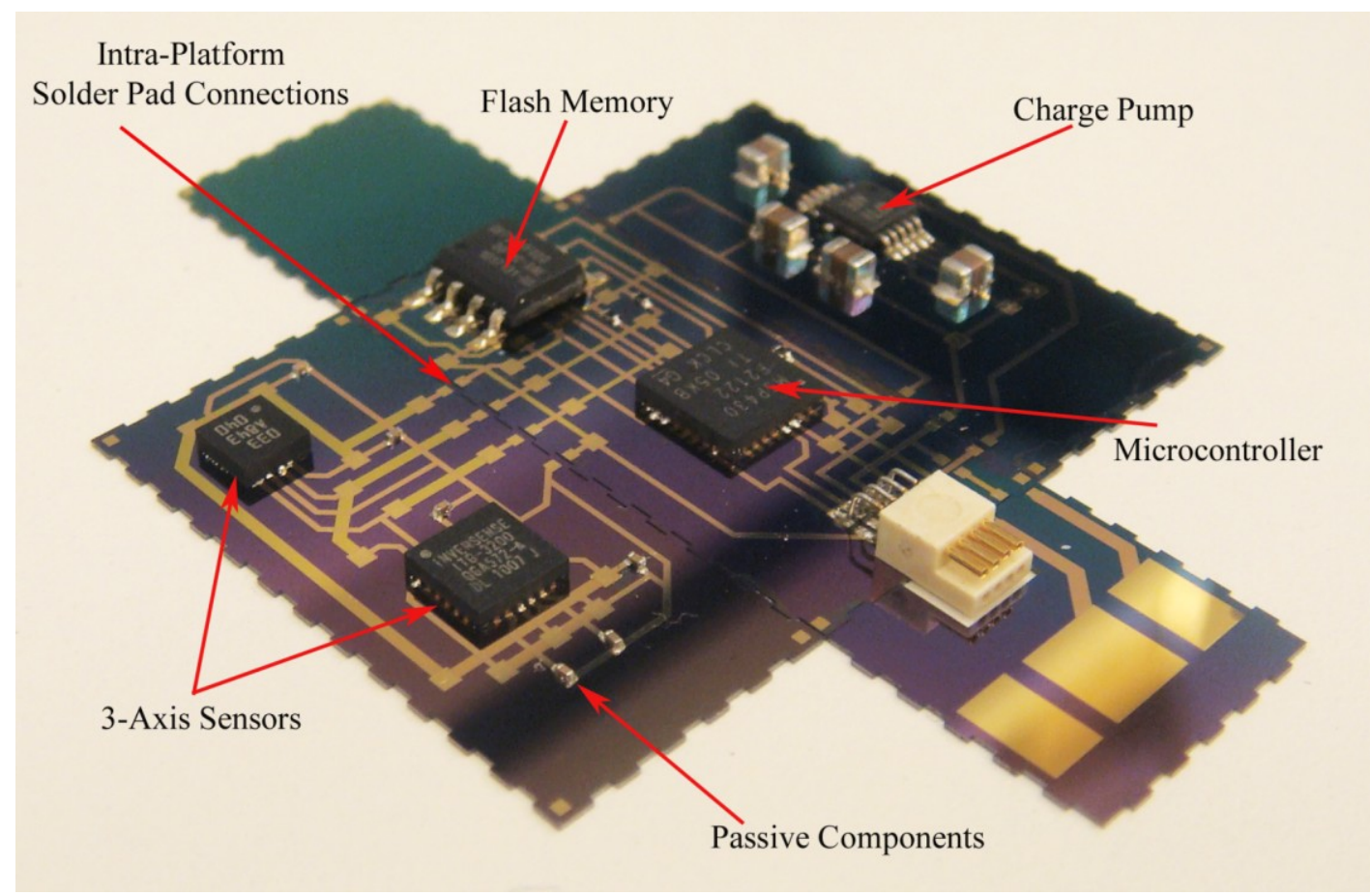

Figure 5.13: Two-dimensional representation of the IMU platform system. 


\section{Chapter 6}

\section{Microsystem Testing and Electrical Properties Assessment}

This chapter discusses the proposed bench top IMU testing equipment and protocol that will be performed to verify the three-axis acceleration and rotation rate data recording and retrieval from the microsystem platform. In addition, a mechanical and electrical reliability assessment is performed to determine the platform's operating parameters.

\subsection{IMU Platform Static Acceleration Testing}

A two-degree-of-freedom test fixture is used to perform all the static acceleration as shown in Fig. 6.1. The test fixture is comprised of two precision rotation stages the Newport RS65 ( $2^{\circ}$ graduations with $0.2^{\circ}$ vernier graduations) and the UTR80 ( $2^{\circ}$ graduations). The entire fixture will be secured to an optical table to minimize vibrations during testing.

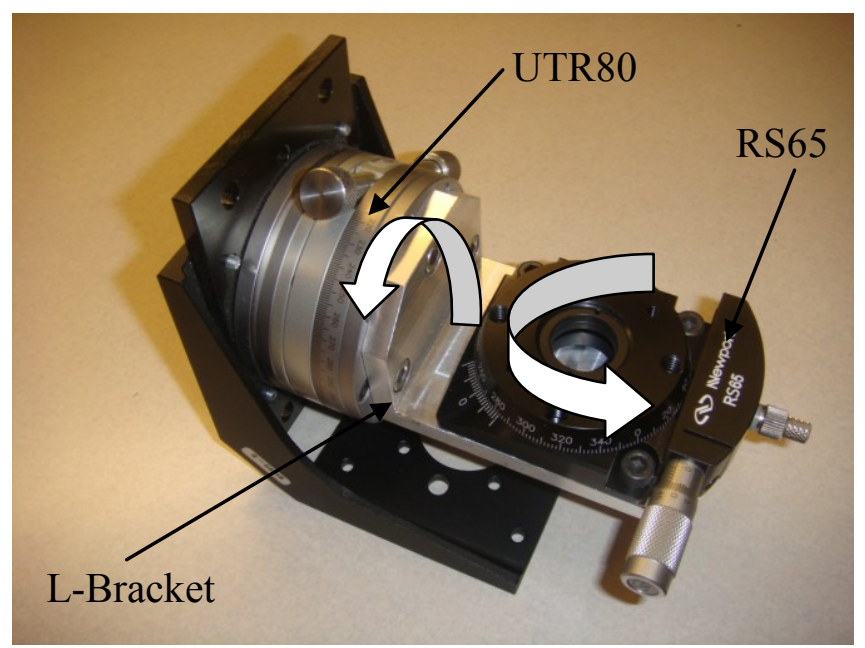

Figure 6.1: Two-degree-of-freedom test fixture for static IMU tests. 
The IMUs under test are secured to a plastic plate using cyanoacrylate which is then secured to the surface of the RS65 rotation stage using screws as shown in Fig. 6.2

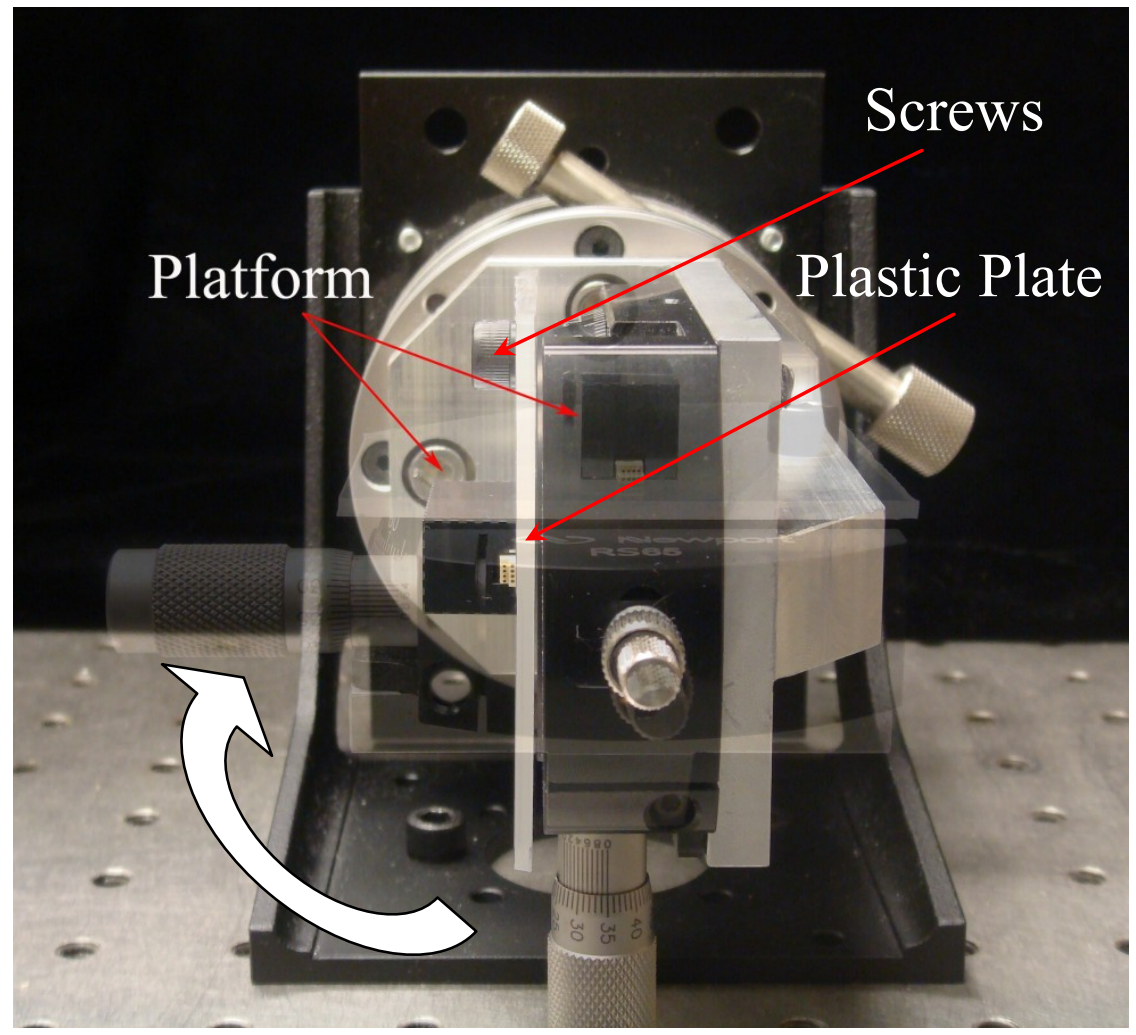

Figure 6.2: Platform secured on two-degree-of-freedom test jig for static testing.

The IMU was subjected to the following test rotation plan shown in Fig. 6.3 which is similar to the standard six-point test for strap down IMUs in which each sensitive axis is alternatively up and down [72]. 


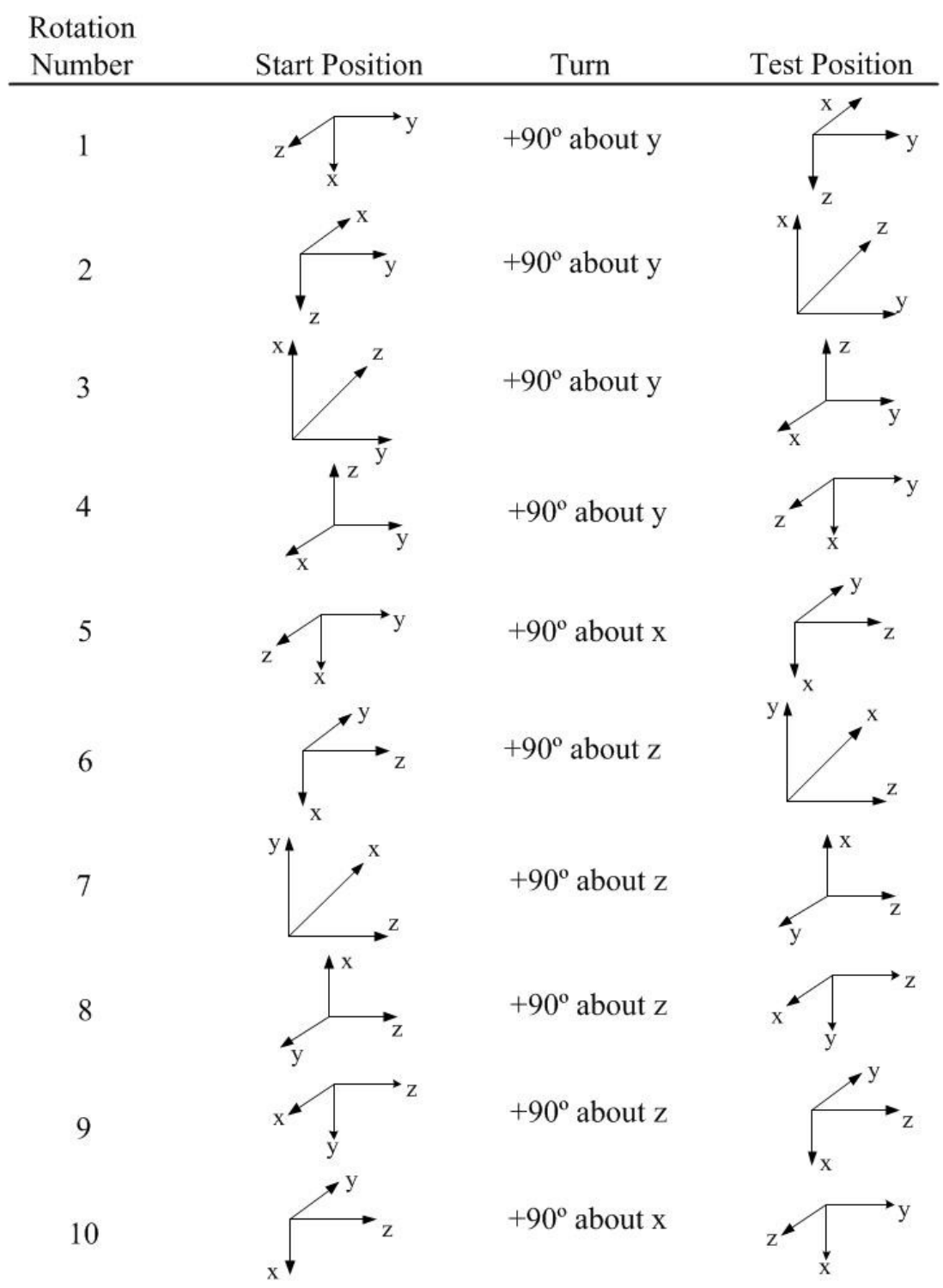

Figure 6.3: Test rotation protocol for the static IMU testing.

Each $90^{\circ}$ rotation was performed manually in a smooth manner, and the test position was held for 10 seconds before proceeding to the next test position. Repeated trials $(n=5)$ were performed and the collected data is plotted in Fig. 6.4 for each of the three recorded acceleration axes. The five repeated trials all show good acceleration data 
repeatability and system reliability as the data matches the expected results based on the platform position for each test segment.
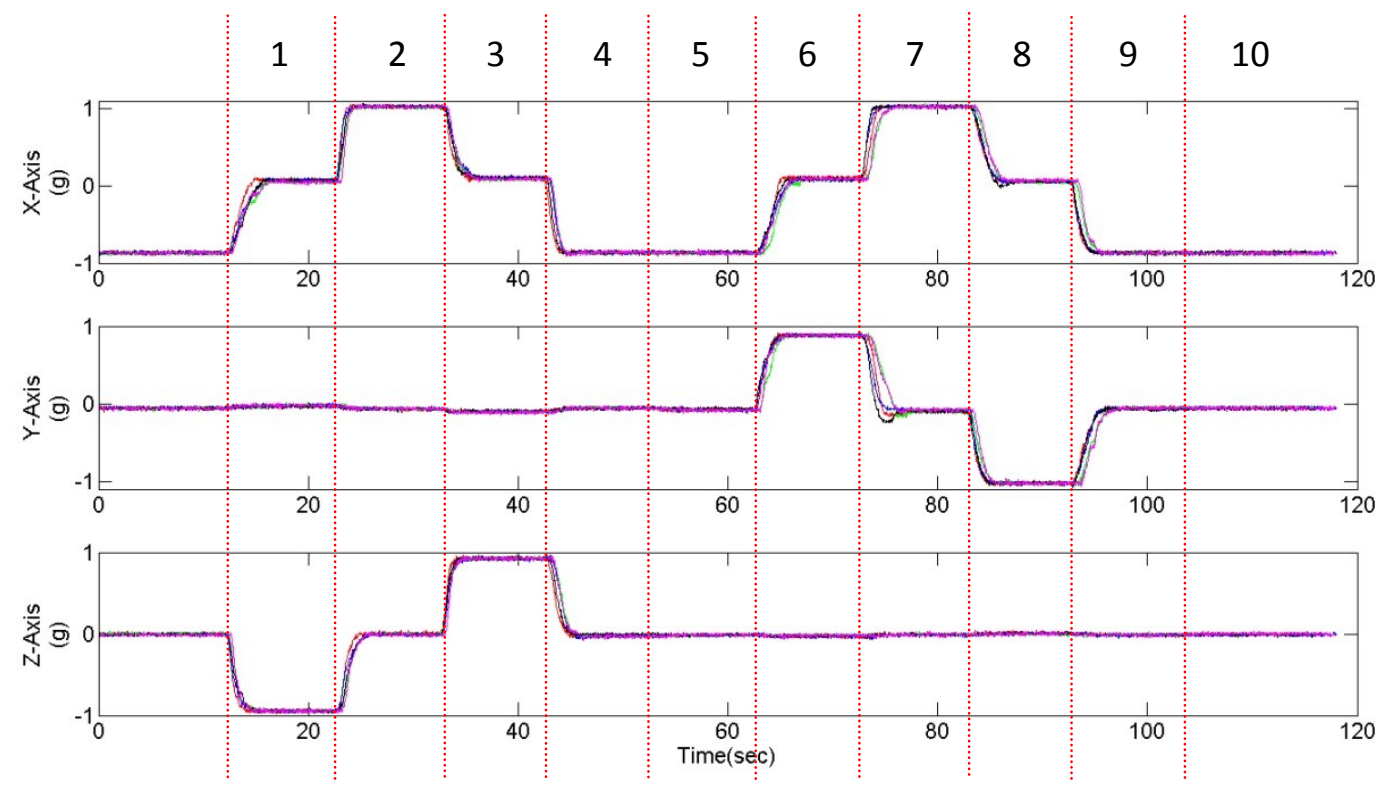

Figure 6.4: Captured platform acceleration data from repeated motion test jig experiments. Numbered periods refer to the rotation test position from Fig. 6.3.

Repeated attempts to acquire the three-axis gyroscope data proved unsuccessful. The problem was identified to be high capacitance values on both the $\mathrm{I} 2 \mathrm{C}$ data and clock bus lines from the microcontroller to the gyroscope. This issue will be addressed in greater detail in Section 6.2 below.

\subsection{Platform Electrical Properties Assessment}

\subsubsection{Resistive/Capacitive Delays on the I2C Clock and Data Bus Lines}

Upon successful assembly of the platform controller and sensor segments together, a test was performed to understand if the microcontroller could acquire the acceleration and rotation rate data via the $\mathrm{I} 2 \mathrm{C}$ bus. The microcontroller was successfully programmed to perform the data-logging function. However, the system would fail because the microcontroller would never receive an acknowledge signal from either 
sensor. Probing the $\mathrm{I} 2 \mathrm{C}$ clock and data lines with an oscilloscope showed longer than expected rise times for both signals. To help reduce the rise times, the existing $4.7 \mathrm{k} \Omega$ I2C clock and data pull-up resisters were replaced with $1.0 \mathrm{k} \Omega$ resistors. Once this change was implemented, the accelerometer data could be acquired but the gyroscope still failed to send an acknowledge signal to the microcontroller.

Using a Keithley $3321 \mathrm{LCZ}$ Meter set at $100 \mathrm{kHz}$ and a parallel circuit type, the capacitance from the $\mathrm{I} 2 \mathrm{C}$ clock and data lines to ground was measured for both the silicon platform and the PCB platform version. The results and the calculated time delays for the different circuit configurations are provided in Table 6.1.

\begin{tabular}{|l|c|c|c|c|}
\hline \multicolumn{1}{|c|}{ IMU Type } & I2C Signal & $\begin{array}{c}\text { Pull-up Resistor } \\
\text { Values }(\mathrm{k} \Omega)\end{array}$ & $\begin{array}{c}\text { Measured } \\
\text { Capacitance }(\mathrm{pF})\end{array}$ & $\begin{array}{c}\text { RC Delay } \\
(\mu \mathrm{s})\end{array}$ \\
\hline Si-Platform & Clock & 4.7 & 768 & 3.61 \\
\hline & Data & 4.7 & 941 & 4.42 \\
\hline & Clock & 1.0 & 768 & 0.768 \\
\hline & Data & 1.0 & 941 & 0.941 \\
\hline PCB-Platform & Clock & 4.7 & 88 & 0.414 \\
\hline & Data & 4.7 & 105 & 0.494 \\
\hline & Clock & 1.0 & 88 & 0.088 \\
\hline & Data & 1.0 & 105 & 0.105 \\
\hline
\end{tabular}

Table 6.1: Calculation of I2C RC delays for different pull-up resistor configurations.

Since the $\mathrm{I} 2 \mathrm{C}$ bus is being operated at a $100 \mathrm{kHz}$ clock frequency, the $\mathrm{I} 2 \mathrm{C}$ clock period is $10 \mu \mathrm{s}$. With the data in Table 5.4 and the Bosch BMA020 three-axis accelerometer datasheet [73] and the InvenSense ITG-3200 three-axis gyroscope datasheet [74], the understanding for the silicon platform communication failures are 
found. First, the BMA020 datasheet requires the $\mathrm{I} 2 \mathrm{C}$ clock high period be at least 2.04 $\mu \mathrm{s}$. With the $4.7 \mathrm{k} \Omega$ pull-up resistors, the rise time is $3.61 \mu$ s resulting in a clock high period of $1.39 \mu \mathrm{s}(5.0 \mu \mathrm{s}-3.61 \mu \mathrm{s})$. Replacing the $4.7 \mathrm{k} \Omega$ resistor with $1.0 \mathrm{k} \Omega$ resistor, produces a clock high period being within specification at $4.232 \mu \mathrm{s}(5.0 \mu \mathrm{s}-0.768 \mu \mathrm{s})$ and hence the successful communication between the accelerometer and the microcontroller. Unfortunately, this change does not fix the gyroscope communication problem because the gyroscope's I2C clock and data rise times are extremely close to the maximum requirement of $1.2 \mu \mathrm{s}$.

From Table 6.1, the I2C clock and data capacitance values for the PCB platform are nearly nine times lower than the silicon platform values even though very similar signal routing patterns were used in both cases. This results in PCB platform rise times being nine times faster than on the silicon platform. To demonstrate the reason for the platform gyroscope fails because of the greater capacitance values on the clock and data lines; $670 \mathrm{pF}$ and $940 \mathrm{pF}$ capacitors were added from the PCB clock and data lines to ground, respectively, to produce the same capacitane values found on the silicon platform. Figure 6.5 shows the extra capacitance put on the PCB I2C signals. The pullup resistors were changed to $1.0 \mathrm{k} \Omega$ to be identical to the silicon platform configuration.

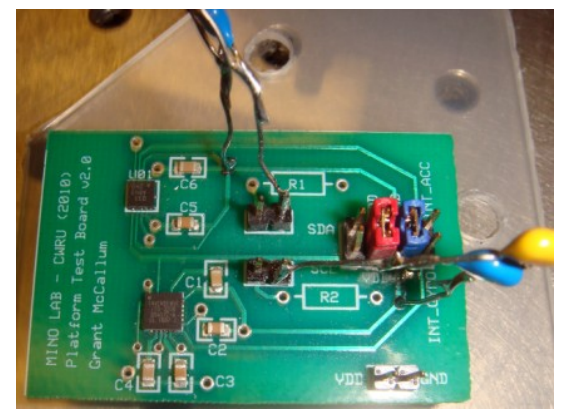

Figure 6.5: PCB platform with additional capacitors to mimic the silicon platform circuit. 
The data-logging program was then loaded on the microcontroller and, as expected, the accelerometer was able to communicate while the gyroscope failed to send an acknowledge signal to the microcontroller. The additional capacitors were removed and the process was repeated for different capacitance loads on the $\mathrm{I} 2 \mathrm{C}$ clock and data lines. To produce a working IMU system, all that was required was to reduce the capacitance on the $\mathrm{I} 2 \mathrm{C}$ clock line to $658 \mathrm{pF}(88 \mathrm{pF}$ original $+560 \mathrm{pF}$ additional). The accelerometer and gyroscope were able to successfully communicate with the microcontroller and produce the data shown in Fig. 6.6 when the PCB platform was manipulated on the two-degree-of-freedom test jig. This implies the silicon platform I2C clock line needs to be reduced by $110 \mathrm{pF}$ to produce a fully working IMU system. This could be achieved by minimizing the $\mathrm{I} 2 \mathrm{C}$ trace lengths or using a thicker, $1.5 \mu \mathrm{m}$, thermal oxide for the isolation layer between the silicon wafer and first metal layer.
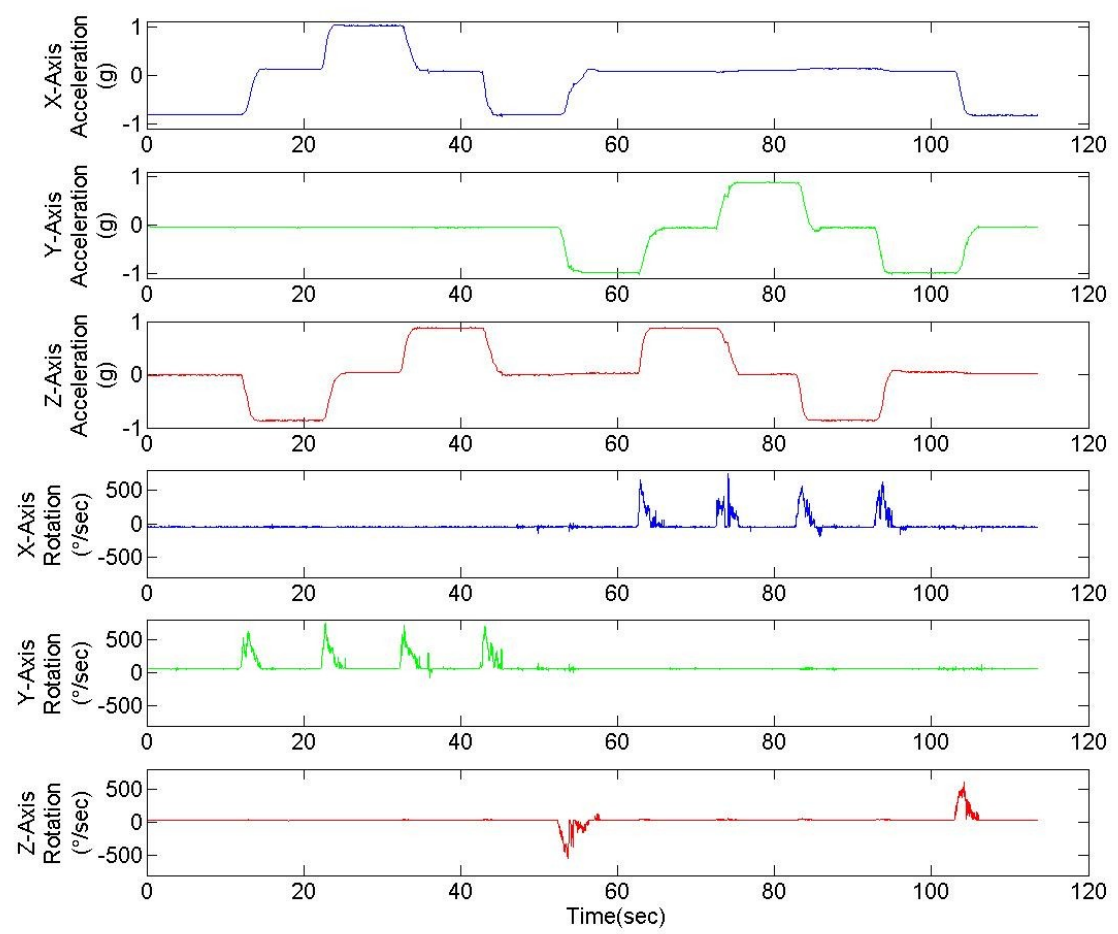

Figure 6.6: Successfully captured PCB platform data with an additional capacitance of $570 \mathrm{pF}$ and $940 \mathrm{pF}$ on I2C clock and data lines, respectively. 


\subsubsection{Power Consumption and Maximum Operating Times}

A Fluke 189 Multimeter was used to measure various currents consumed by the platform components during the data-logging operation as shown in Fig. 6.7. The entire system current consumption measured from the battery terminals was found to be 15.947 $\mathrm{mA}$, and the largest current consuming component was the three-axis gyroscope with 6.021 mA. The small value I2C pull-up resistors lead to high current consumption on the I2C clock and data lines which could be significantly reduced by careful control of the capacitance values on these bus lines during platform system design.

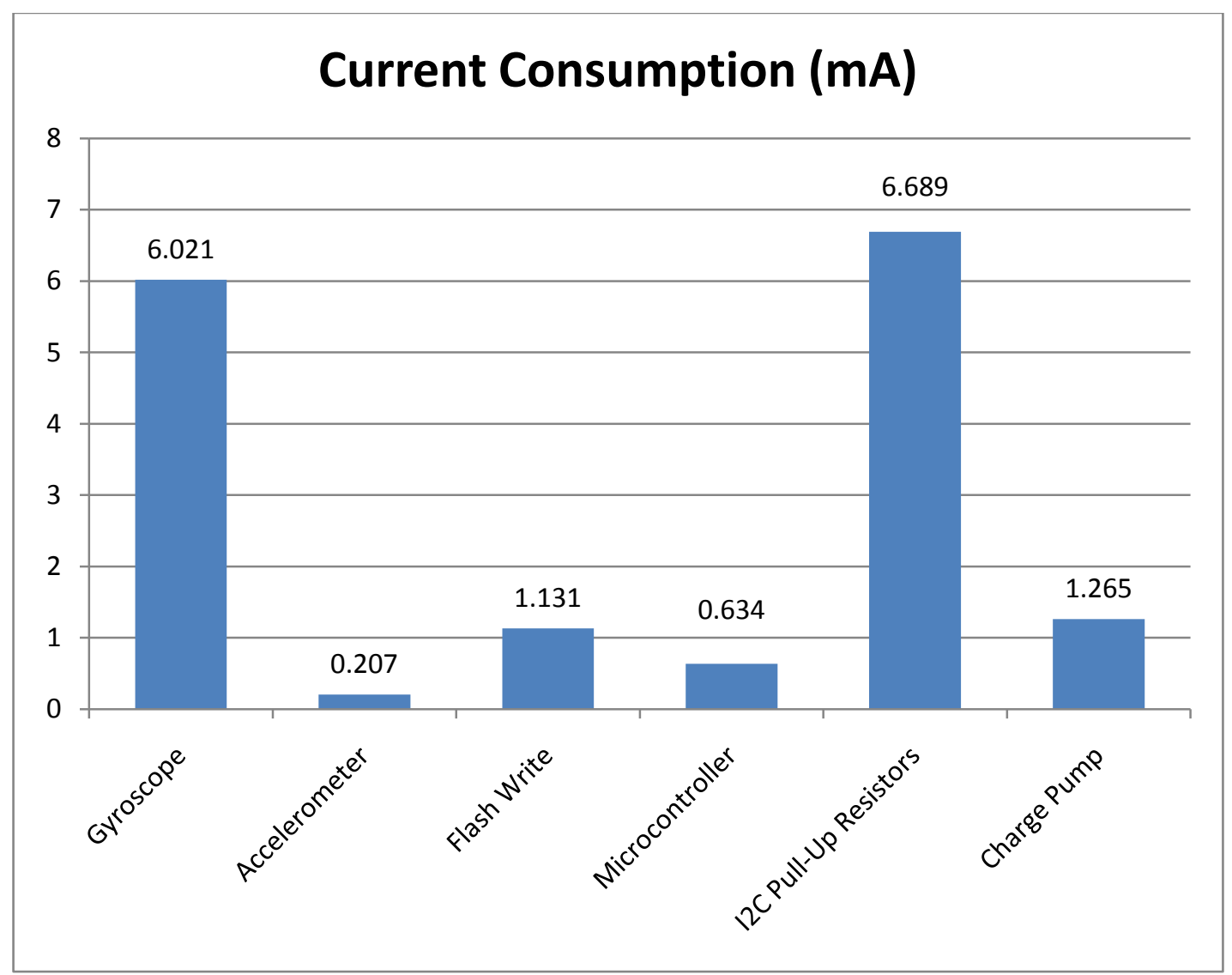

Figure 6.7: Current consumption for different platform components during data-logging operation at $100 \mathrm{~Hz}$ data-acquisition rate. 
Based on the current consumption numbers measured above, the total operating lifetime of the platform before reaching the charge pump $1.8 \mathrm{~V}$ minimum input voltage requirement is 90.24 minutes, or approximately 1.5 hours. This is well over the 3.64 minutes required to completely fill the 2 Mbit Flash memory with sensor data as discussed in Chapter 5. The microcontroller can be configured to sample and store all the sensor data at its lowest rate of $2 \mathrm{~Hz}(0.5$ seconds). Table 6.2 shows the maximum possible data-logging time the platform can accommodate depending on 2, 100 and 145 Hz sampling rates and Flash memory sizes of 2, 4, 816 and 32 Mbit that are footprint compatible with the existing 2 Mbit Flash memory used on the platform.

\begin{tabular}{|c|c|c|c|c|c|}
\hline $\begin{array}{c}\text { Sampling } \\
\text { Rate }\end{array}$ & \multicolumn{5}{|c|}{ Flash Memory Size } \\
\hline $2 \mathrm{~Hz}$ & $3 \mathrm{hrs}, 2 \mathrm{~min}$ & $6 \mathrm{hrs}, 4 \mathrm{~min}$ & $12 \mathrm{hrs}, 7 \mathrm{~min}$ & $24 \mathrm{hrs}, 16 \mathrm{~min}$ & $48 \mathrm{hrs}, 33 \mathrm{~min}$ \\
\hline $100 \mathrm{~Hz}$ & $3 \mathrm{~min}, 38 \mathrm{sec}$ & $7 \mathrm{~min}, 17 \mathrm{sec}$ & $14 \mathrm{~min}, 34 \mathrm{sec}$ & $29 \mathrm{~min}, 8 \mathrm{sec}$ & $58 \mathrm{~min}, 15 \mathrm{sec}$ \\
\hline $145 \mathrm{~Hz}$ & $2 \mathrm{~min}, 31 \mathrm{sec}$ & $5 \mathrm{~min}, 1 \mathrm{sec}$ & $10 \mathrm{~min}, 2 \mathrm{sec}$ & $20 \mathrm{~min}, 5 \mathrm{sec}$ & $40 \mathrm{~min}, 11 \mathrm{sec}$ \\
\hline
\end{tabular}

Table 6.2: Maximum platform data-logging duration for 2, 100 and $145 \mathrm{~Hz}$ sampling rates and 2, 4, 8, 16, and 32 Mbit Flash memory sizes.

Therefore, with the existing platform configuration, the platform can collect data for the three-hour duration of battery life at a rate of $2 \mathrm{~Hz}$. At the current $100 \mathrm{~Hz}$ platform sampling rate, approximately 58 minutes of sensor data can be collected if the largest Flash memory configuration is placed on the platform. The microcontroller takes 6,813 total core clock cycles to retrieve all six axes of data and store them to the Flash memory. At a core clock rate of $1.0 \mathrm{MHz}$, the maximum sampling rate is $146.78 \mathrm{~Hz}$. Therefore, $145 \mathrm{~Hz}$ is chosen as the maximum sensor data sampling rate to leave overhead for correct operation. 


\subsection{Platform Antenna Integration for Wireless Communication}

The following section investigates adding antenna features to determine if it is feasible as the platform volume decreases and, if so, where should the research be focused to achieve the integration of wireless capability.

Recently, researches have demonstrated planar UHF band antennas for RFID applications $[75,76]$. These antennas operate in the $433 \mathrm{MHz}$ and $868 \mathrm{MHz}$ frequencies and are made with different substrate materials such as FR-4 and RO4003C, a high frequency material that has a low dissipation factor. The antenna sizes in this frequency range are $20 \times 39 \times 1.6 \mathrm{~mm}^{3}$ and $24 \times 11 \times 3 \mathrm{~mm}^{3}$, respectively, which is relatively large for the future $1 \mathrm{~mm}^{3}$ platform cube. To reduce the planar footprint of the antenna, research on a $915 \mathrm{MHz}$ meander line based antenna is created on a liquid crystal polymer and folded into a cube shape as shown in Fig. 6.8 [77]. One can imagine the same methodology used for the existing silicon-based platform.

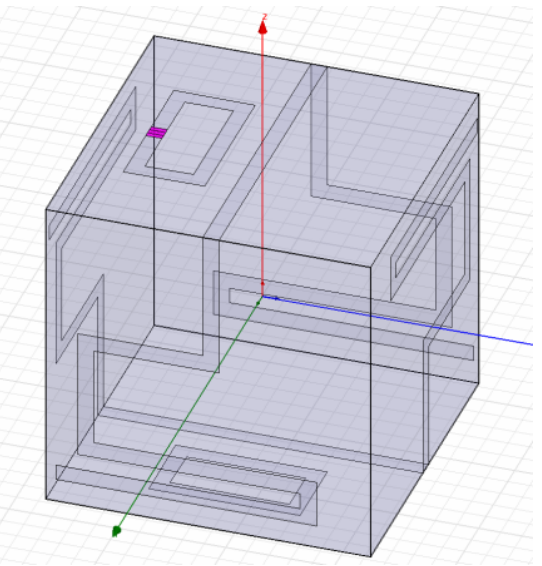

Figure 6.8: Three-dimensional antenna design for a non-uniform meander line [77]. 
The physical antenna size can be reduced with higher operating frequencies and recent research has looked at the millimeter wavelengths designs $(17 \mathrm{GHz}-135 \mathrm{GHz})$. A $17 \mathrm{GHz}$ notch antenna was fabricated on $100 \mu \mathrm{m}$ thick polyimide flexible laminate and was $20 \times 10 \mathrm{~mm}^{2}$ in planar size for use in a body area network application [78].

However, antennas that operate in the $50 \mathrm{GHz}$ to $60 \mathrm{GHz}$ range are the main focus for most research efforts. A $50 \mathrm{GHz}$ adaptive micro-patch antenna was fabricated on a silicon substrate and controlled by MEMS-based DMTL phase shifters which measured $1.1 \times 1.1 \mathrm{~mm}^{2}$ [79]. Research efforts with $60 \mathrm{GHz}$ combination slot-patch antennas include Integrated Passive Devices (IPD ${ }^{\mathrm{TM}}$ ) technology from STMicroelectronics for the antenna substrate [80] and a multilayer design with patch coupling and cavity resonance leads to a compact and wideband antenna solution [81]. The two mentioned $60 \mathrm{GHz}$ antennas have planar dimensions of $1.71 \times 2.0 \mathrm{~mm}^{2}$ and $2.5 \times 2.5 \mathrm{~mm}^{2}$, respectively. Finally, a $135 \mathrm{GHz}$ co-planar patch antenna has been microfabricated on silicon wafers with polymer-filled cavities as shown in Fig. 6.9 [82] which has a planar dimensions of $600 \times 700 \mu \mathrm{m}^{2}$. Per the earlier discussion, antennas designed for this operating frequency would enable wireless communications for the $1.5 \mathrm{~mm}^{3}$ platform as one could fabricate the antenna structures on a single platform component face. 


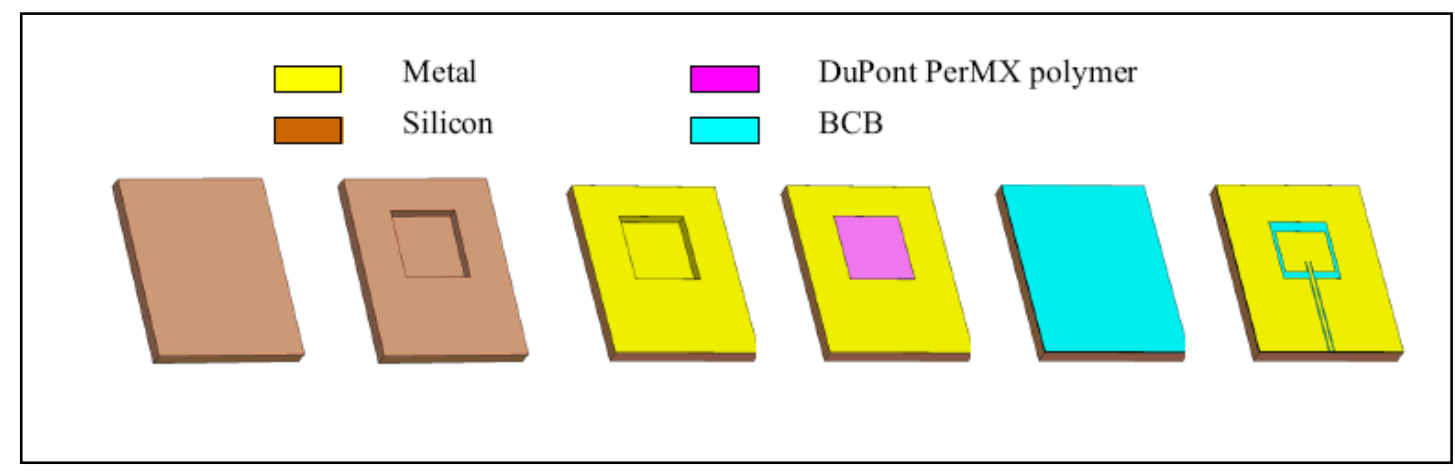

Figure 6.9: $135 \mathrm{GHz}$ co-planar patch antenna with polymer-filled cavity [82].

A recent survey of the state-of-the-art antennas on silicon for millimeterwave applications show that the best designs that demonstrate high gain, high efficiency percentage and high bandwidth are created using a BiCMOS SiGe process or CMOS SOI wafer substrates [83]. Adopting these design strategies reduces the effect caused by the intrinsic resistivity of the silicon substrate, which absorbs a significant part of the energy emanating from the antenna.

From the current research results of millimeterwave antenna designs and the progress made with terahertz CMOS integrated circuits [84] to provide the wireless frontend circuitry, the future realization of wireless communications for the $1.5 \mathrm{~mm}^{3}$ platform structure is possible. 


\section{Chapter 7}

\section{Conclusion and Future Work}

A unique 3-D silicon platform has been successfully created using standard microfabrication techniques; it houses all the necessary electronic components and the required power supply to realize a functional IMU. Mesoscale platform versions are created using PCBs to enable the software development of the microcontroller system code and as a proof-of-concept.

The 3-D cubic/rectilinear platform structure is created from planar component pieces, which are then assembled to electrically route internal signals between different platform pieces via orthogonal solder joints. The planar components can be situated at a right angle to other components, increasing functionality density and reducing the system's planar footprint. In addition, the platform enables diverse system integration where individual planar component pieces can be fabricated using different substrates, materials and/or microfabrication processes to create a heterogeneous system once assembled.

The key observations resulting from this work include the weak mechanical strength exhibited when the more complicated "Hook-Hole" assembly strategy is implemented, when compared to the simple interdigitated teeth connector method. The platform's mechanical strength can be further increased by reducing the overall volume and maintaining a cubic shape. 
For identical electrical signal routing patterns, the capacitive loading on the platform measured nearly an order of magnitude greater compared to the PCB capacitive loading effects. Therefore, extreme care must be given to time critical platform signals such as the $\mathrm{I} 2 \mathrm{C}$ communication bus between the microcontroller and the sensors. This could have significant impact on the operation of the platform system, which occurred with the three-axis gyroscope communication.

Lastly, the volume of the platform is largely determined by the size of the embedded power source. The small coin cell batteries used in the current platform architecture were possible only with the addition of the charge pump circuitry, which provided the necessary drive current. Alternative power sources and schemes will have to be developed to further reduce the overall volume of the platform.

\subsection{Future Work}

As mentioned above, the true power of this platform is realized when a design using different substrate materials is considered. Assuming all the platform substrate materials can be micromachined to have one of the connector methods mentioned herein, system designers are free to use materials that best accomplish the specific system function. An example of a hybrid platform is shown in Fig. 7.1 which contains a sensor segment potentially made from a silicon carbide wafer substrate, an antenna segment fabricated using a silicon-on-insulator (SOI) substrate with thru-silicon vias (TSV) to a transceiver IC soldered to the backside of the segment. The transparent optical properties of micromachined glass can be used to create platform segments in which solar cells for power generation or infra-red (IR) sensors for imaging applications could be bonded to the inside platform surface as shown in Fig. 7.1. Finally, a custom ASIC segment 
fabricated on a standard silicon wafer using a deep-submicron process technology is shown.

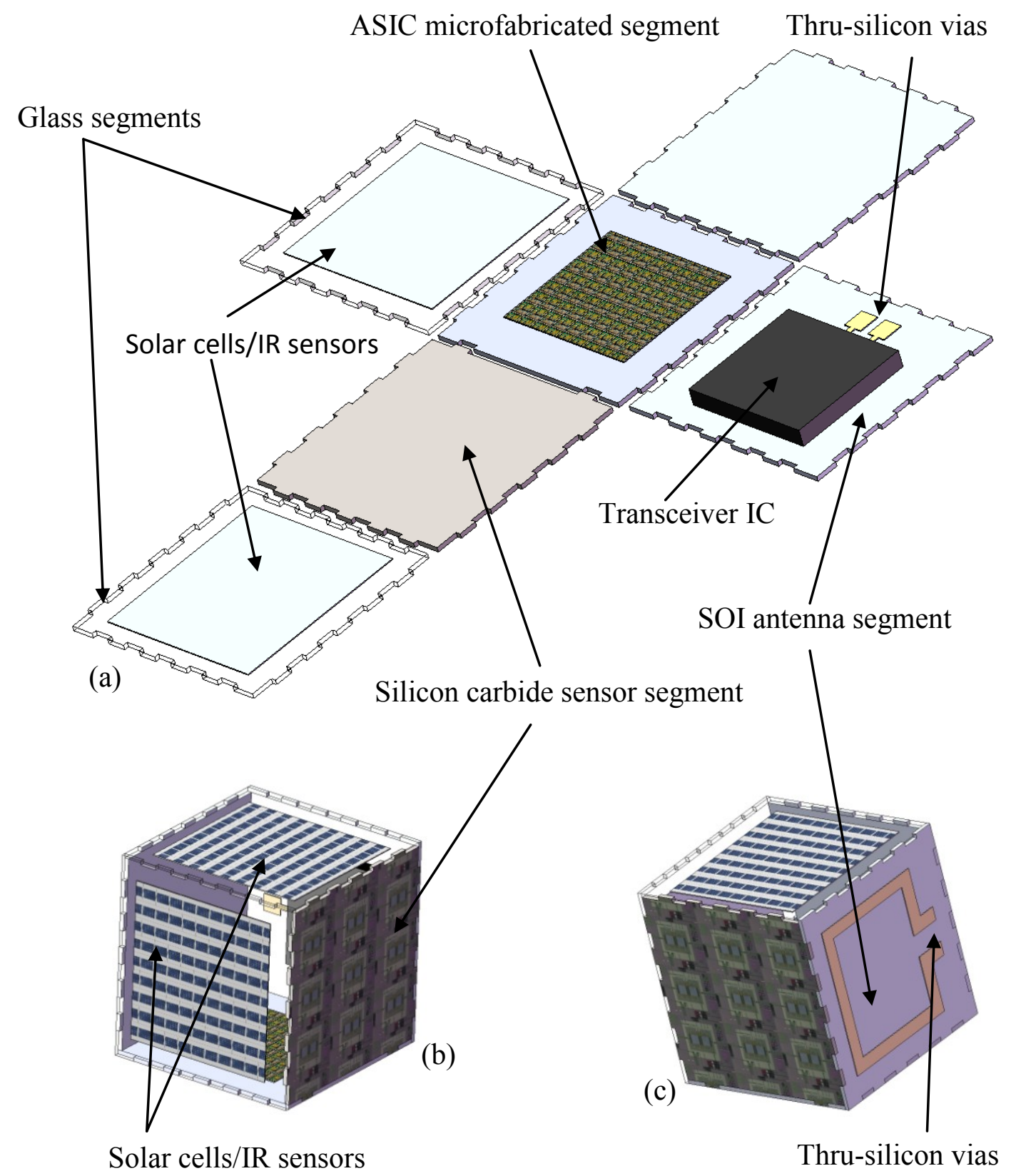

Figure 7.1: Concept drawing of a hybrid substrate material platform configuration: (a) 2D unassembled platform segments; (b) 3-D assembled platform showing the $\mathrm{SiC}$ sensor segment and two glass segments; (c) 3-D assembled platform showing the $\mathrm{SiC}$ sensor segment, SOI antenna segment and glass segment with solar cells for power generation. 
Although not shown in Fig. 7.1, orthogonal solder joints would be used to electrically and mechanically connect the platform segments together. The orthogonal solder joint work could be further advanced so that a completely enclosed and secure platform structure is realized. One idea is to pre-tin all the orthogonal solder pad locations on the Top platform segment and pad locations on the platform sidewall segments. When the Top platform segment is placed on the platform structure these pretinned solder features will touch each other. Then the entire platform structure could be heated so these touching solder joint features reflow and orthogonal solder joints are created to mechanically and electrically connect all six platform segments.

The platform's modularity provides a means to easily replace functions and features by simply exchanging a platform component side with another even if the substrate material is different. This allows any future platform development to proceed in parallel as different functional aspects of the system can be partitioned on different platform segments. For instance, the existing $12 \times 12 \times 25 \mathrm{~mm}^{3}$ platform has two internal sides that are not used at all. It is easy to imagine that one of these component sides has an antenna fabricated on one-side and transceiver circuitry on the other to add wireless communication to the platform.

The platform presented herein significantly increases the design possibilities allowing greater flexibility in materials and devices used in the microsystem. One might argue that is also true for 3-D die stacked systems. However, 3-D die stacking limits only one die to interface directly to the outside environment. The 3-D platform presented here allows five die faces (i.e., segments) to directly interface with the external environment. This allows for simultaneous sensing, power harvesting (i.e., solar cells) and wireless 
communication in a single, compact structure. Of course, to fully achieve the packaging functionality, media-compatible materials would need to be integrated on the surfaces exposed to the media which surrounds the platform. 


\title{
Appendix A: Microfabrication Details
}

\section{CASE WESTERN RESERVE UNIVERSITY FABRICATION DETAILS}

\author{
A.1.1 Positive Photoresist AZ9260 (DRIE Processing)
}

- HMDS Application on Silicon then Dehydration

- Spin AZ9260 2200RPM for 60 seconds

- Hotplate Softbake for 1 min 50 seconds at $110^{\circ} \mathrm{C}$

- Align and expose for 29 seconds at $23 \mathrm{~mW} / \mathrm{cm}^{2}$, wec 2.0 , hard contact

- Develop with (1:3) AZ400K and DI water for 4 minutes

- Rinse with DI water for 1-2 minutes

A.1.2 Positive Photoresist AZ9245 (5 $\mu \mathrm{m}$ thick photoresist) Metal Lift-Off

- HMDS Application on Silicon Dioxide then Dehydration

- Spin AZ9245 3500RPM for 30 seconds

- Hotplate Softbake for one (1) minute at $110^{\circ} \mathrm{C}$

- Align and expose for 20 seconds at $23 \mathrm{~mW} / \mathrm{cm}^{2}$, wec 2.0 , hard contact

- Develop with (1:3) AZ400K and DI water for 2 minutes 30 seconds

- Rinse with DI water for 1-2 minutes

\section{A.1.3 RIE Recipes}

- RIE Etch through LPCVD Silicon Dioxide Layer

$$
\text { - Gas \#1: } \quad \text { Oxygen } \quad 24.9 \mathrm{sccm}
$$


- Gas \#2: $\quad$ Helium $10 \mathrm{sccm}$

- Gas \#3: $\quad$ Freon-23 $10 \mathrm{sccm}$

- Pressure 350 mTorr

- Power $\quad 450 \mathrm{~W}$

- Rate $\quad 10 \mathrm{~min}$ for $3000 \AA$

- RIE Etch through PECVD Oxide

- Gas \#2: $\quad$ Helium $10 \mathrm{sccm}$

- Gas \#3: $\quad$ Freon-23 4 sccm

- Pressure 200 mTorr

- Power $\quad 450 \mathrm{~W}$

- Rate 8 min for $3000 \AA$

A.1.4 DRIE Recipe

- General

- Start $=>$ Etch ; End $=>$ Passivate

- Etch Cycle Times: $\quad 9.7 \mathrm{sec}, \quad 10.7 \mathrm{sec}, \quad 11.7 \mathrm{sec}$

- Passivate Cycle Times: 7sec, 7sec, $7 \mathrm{sec}$

- Pressure

- Base 0.0mTorr; trip 94.0mTorr; Manual APC $=84 \%$

- Gases

- Etch Gases

$-\mathrm{SF}_{6} \quad 130 \mathrm{sccm} \quad ; 50 \%$ tol

$-\mathrm{O}_{2} \quad 13 \mathrm{sccm} \quad ; 10 \%$ tol

- Passivation Gas

$-\mathrm{C}_{4} \mathrm{~F}_{8} 85 \mathrm{sccm} \quad ; 10 \%$ tol

- RF

- 13.56 MHz Coil 
- Power: 600W (etch) ; 600W (passivate)

- Matching $=$ Auto; Load $=50 \%:$ Tune $=50 \%$

$-13.56 \mathrm{MHz}$ Platen

- Power: 12W (etch); 0W (passivate)

- Matching $=$ Auto; Load $=50 \% ;$ Tune $=50 \%$

- Electrode 13.56 MHz; Range 0-300W

A.1.5 PECVD Oxide TEOS Recipe (1800 ̊̊/min deposition rate)

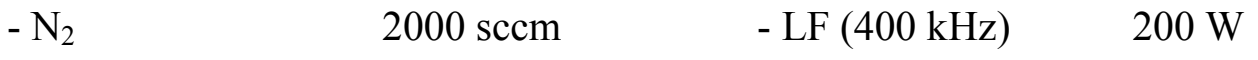

$\begin{array}{llll}-\mathrm{O}_{2} & 1000 \mathrm{sccm} & \text { - Pressure } & 3 \text { Torr }\end{array}$

$\begin{array}{llll}\text { - TEOS } & 1000 \mathrm{sccm} & \text { - Temperature } & 365^{\circ} \mathrm{C}\end{array}$

- HF (13.56 MHz) $\quad 1000 \mathrm{~W}$

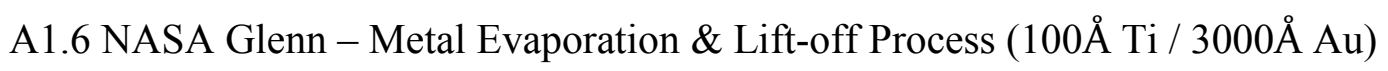

- Cooled for two hours using substrate chiller under the gold target in a 6" MRC vacuum system.

- Heat exchanger cools to $-20^{\circ} \mathrm{C}$.

- Rotated the wafer under the 6" titanium target and deposited for 3 minutes at 100 watts using five nines argon gas at $6 \mathrm{~m} /$ Torr $(100 \mathrm{~A})$.

- Then rotated the wafer back under the gold target. Waited 5 minutes, then deposit gold for 10 minutes at 100 watts using five nines argon at $6 \mathrm{~m} /$ Torr (3,000A). Turned off pump and unloaded.

- Lift-off gold film using acetone in ultrasonic cleaner for 10 minutes. 


\section{Appendix B}

\section{C-language Program for Platform Data-Logging}

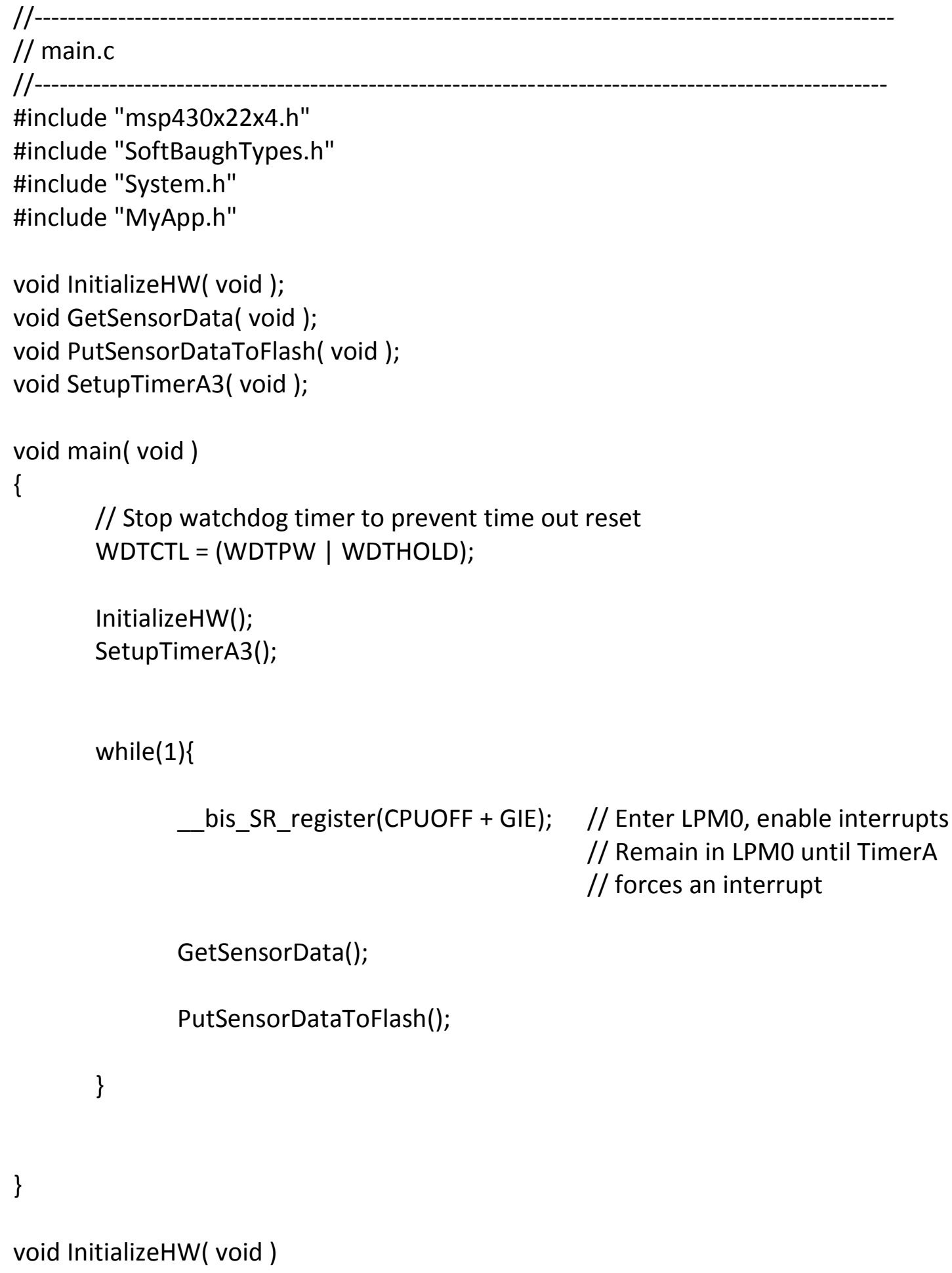




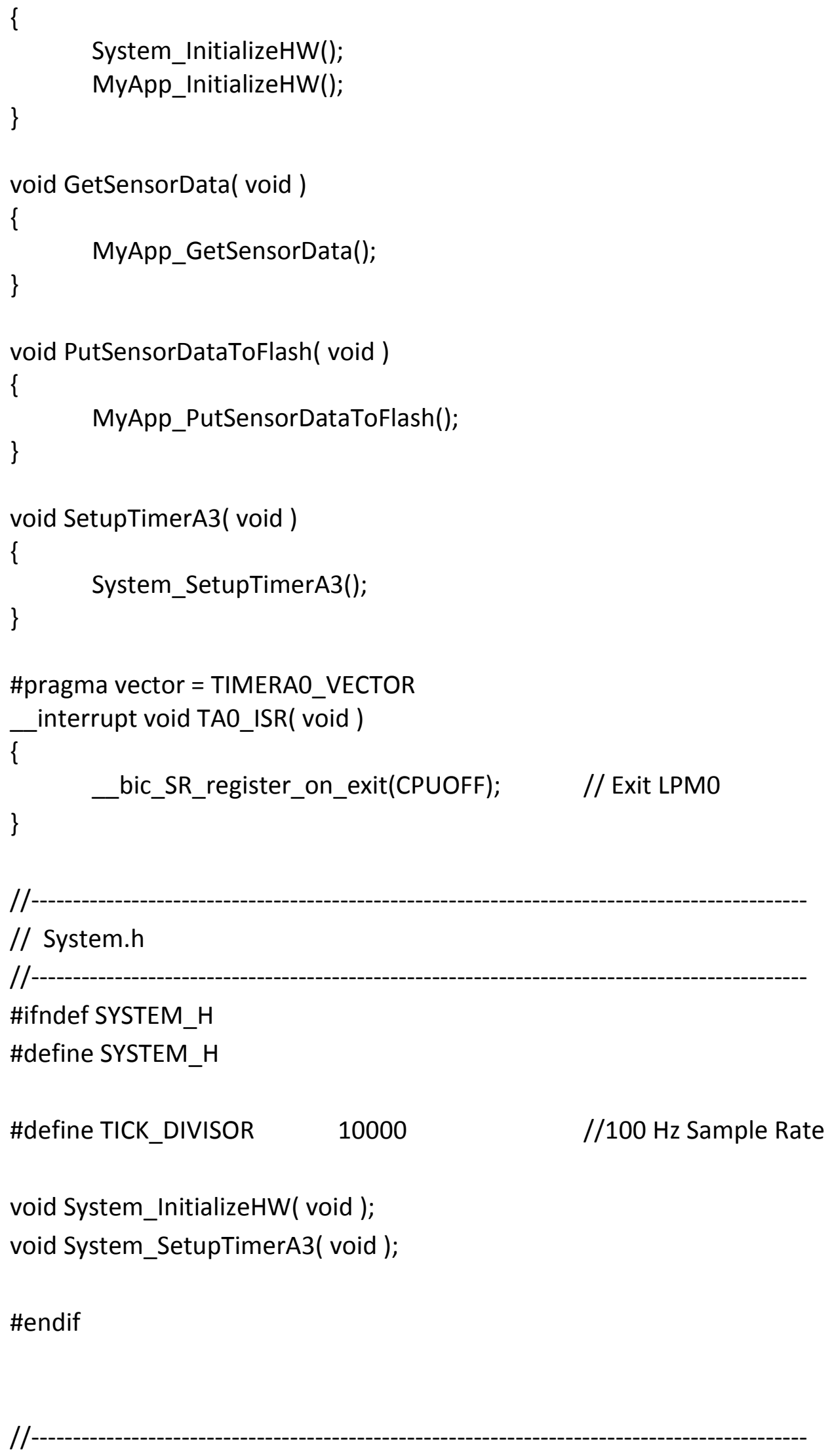




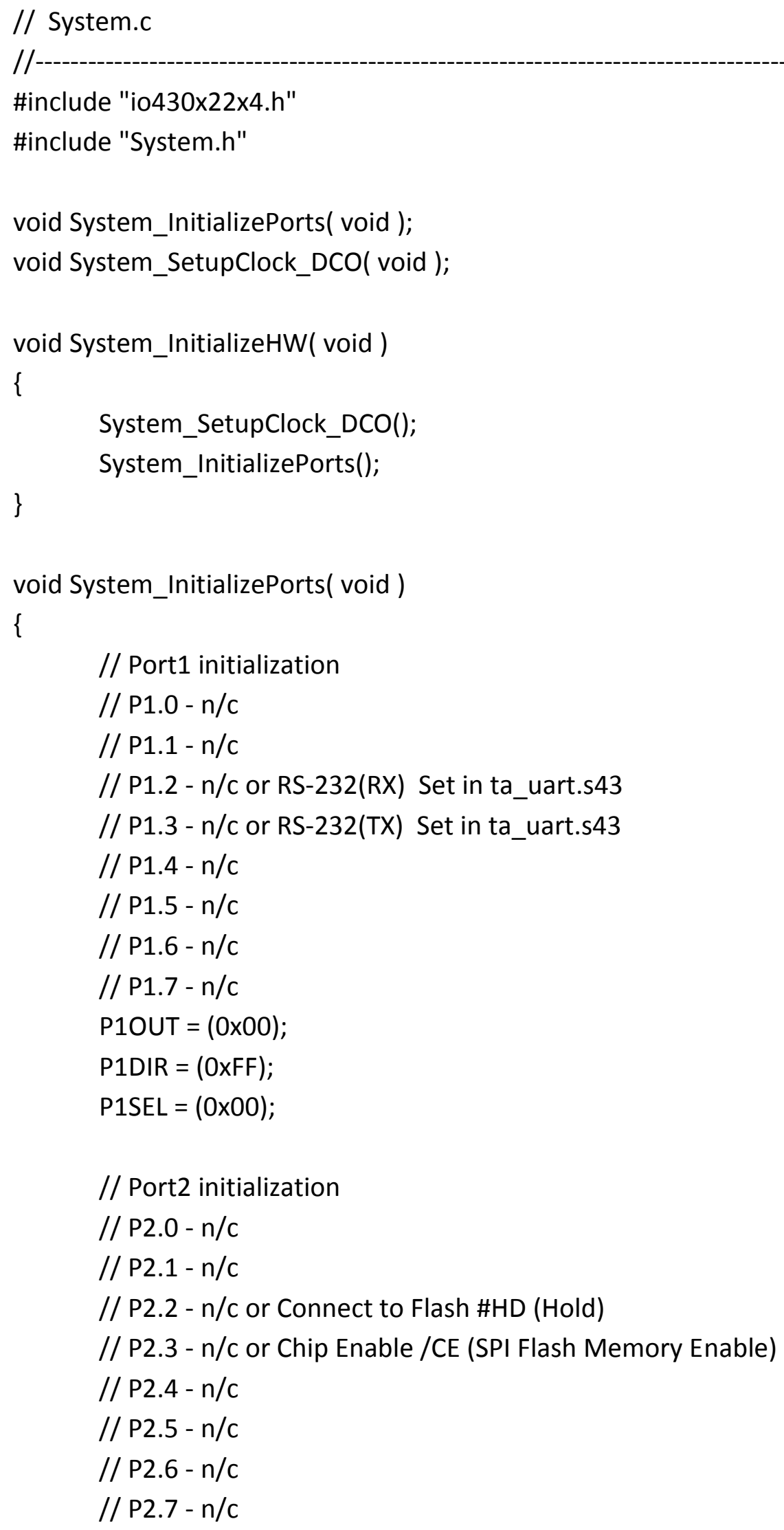




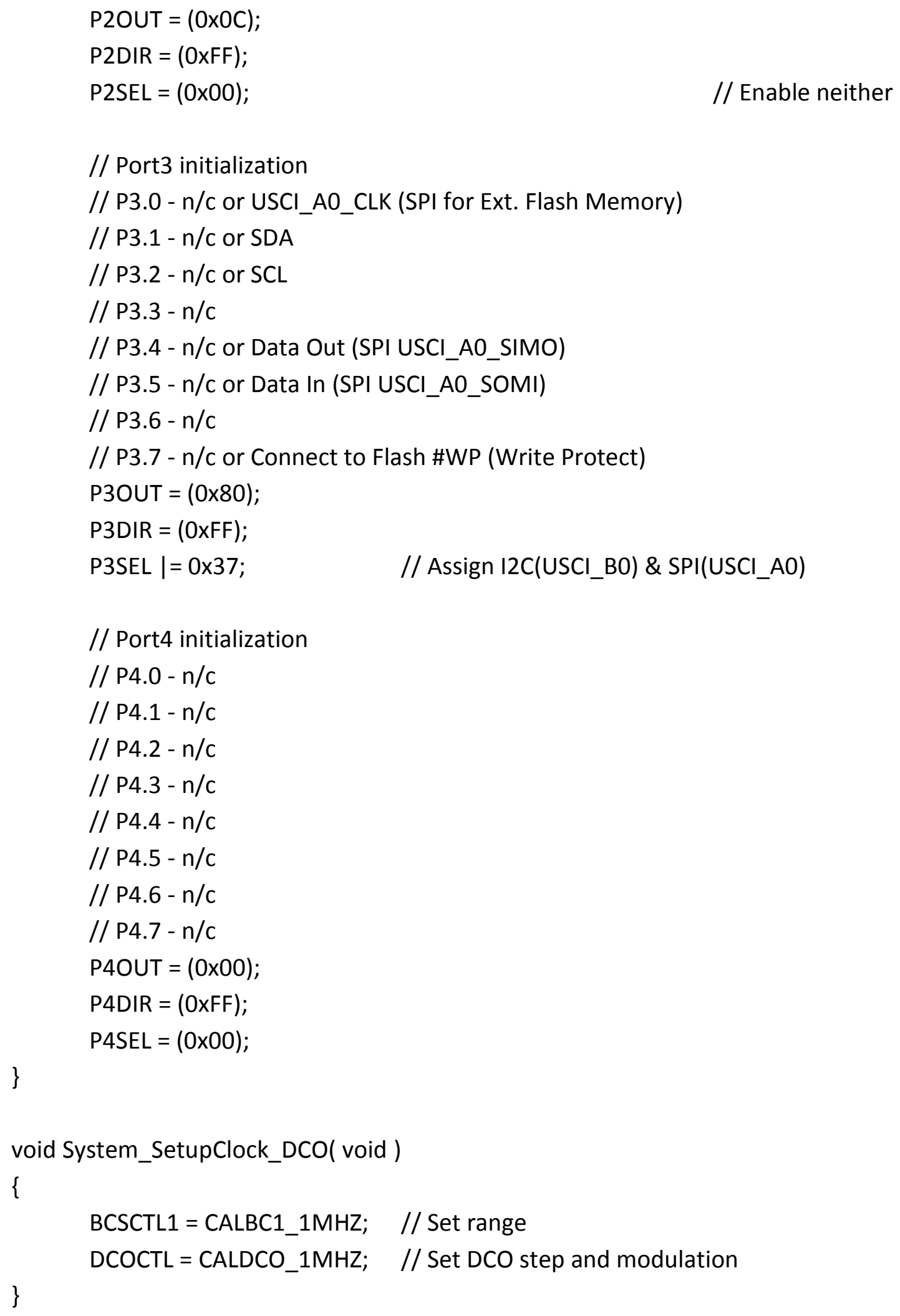


void System_SetupTimerA3( void)

\{

// Prepare the divisor

TACCRO = (TICK_DIVISOR-1);

// Up Mode from ACLK

TACTL = (TASSEL_2|ID_0|MC_1|TACLR);

// Interrupt on CCRO

TACCTLO = $($ CCIE $)$;

\}

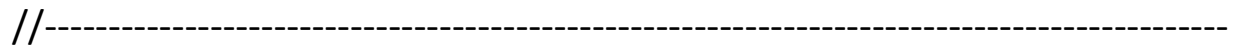

// myApp.h

$/ /--$

\#ifndef MY_APP_H

\#define MY_APP_H

void MyApp_InitializeHW( void);

void MyApp_GetSensorData( void);

void MyApp_PutSensorDataToFlash( void );

\#endif

$/ /-$

// myApp.c

$/ /-$

\#include "io430x22x4.h"

\#include "SoftBaughTypes.h"

\#include "stdint.h"

\#include "System.h"

\#include "MyApp.h"

\#define ACCEL_SLAVE_ADDRESS $\quad 0 \times 38$

\#define GYRO_SLAVE_ADDRESS 0x68

\#define ACCEL_CTRL_BYTE $\quad 0 \times 02$ 


$\begin{array}{ll}\text { volatile unsigned char AccXBuffer[8]; } & \text { // Allocate 8B of RAM } \\ \text { volatile unsigned char AccYBuffer[8]; } & \text { // Allocate 8B of RAM } \\ \text { volatile unsigned char AccZBuffer[8]; } & \text { // Allocate 8B of RAM } \\ \text { unsigned char *PAccXData; } & \text { // Pointer to Accel X Data } \\ \text { unsigned char *PAccYData; } & \text { // Pointer to Accel Y Data } \\ \text { unsigned char *PAccZData; } & \text { // Pointer to Accel Z Data } \\ & \\ \text { volatile unsigned int k; } & \\ \text { volatile unsigned char GyrXBuffer[8]; } & \text { // Allocate 8B of RAM } \\ \text { volatile unsigned char GyrYBuffer[8]; } & \text { // Allocate 8B of RAM } \\ \text { volatile unsigned char GyrZBuffer[8]; } & \text { // Allocate 8B of RAM } \\ \text { unsigned char *PGyrXData; } & \text { // Pointer to Gyro X Data } \\ \text { unsigned char *PGyrYData; } & \text { // Pointer to Gyro Y Data } \\ \text { unsigned char *PGyrZData; } & \text { // Pointer to Gyro Z Data }\end{array}$

void MyApp_InitializeHW_Accel( void );

void MyApp_InitializeHW_Gyro( void );

void MyApp_InitializeHW_Flash( void);

void MyApp_PutDummyDataToFlash( void );

void MyApp_InitializeHW( void )

\{

// MyApp_InitializeHW_Accel();

MyApp_InitializeHW_Gyro();

MyApp_InitializeHW_Flash();

MyApp_PutDummyDataToFlash();

\}

void MyApp_InitializeHW_Accel( void )

\{

\}

void MyApp_InitializeHW_Gyro( void )

\{ 
// Configure the Gyro via I2C Interface

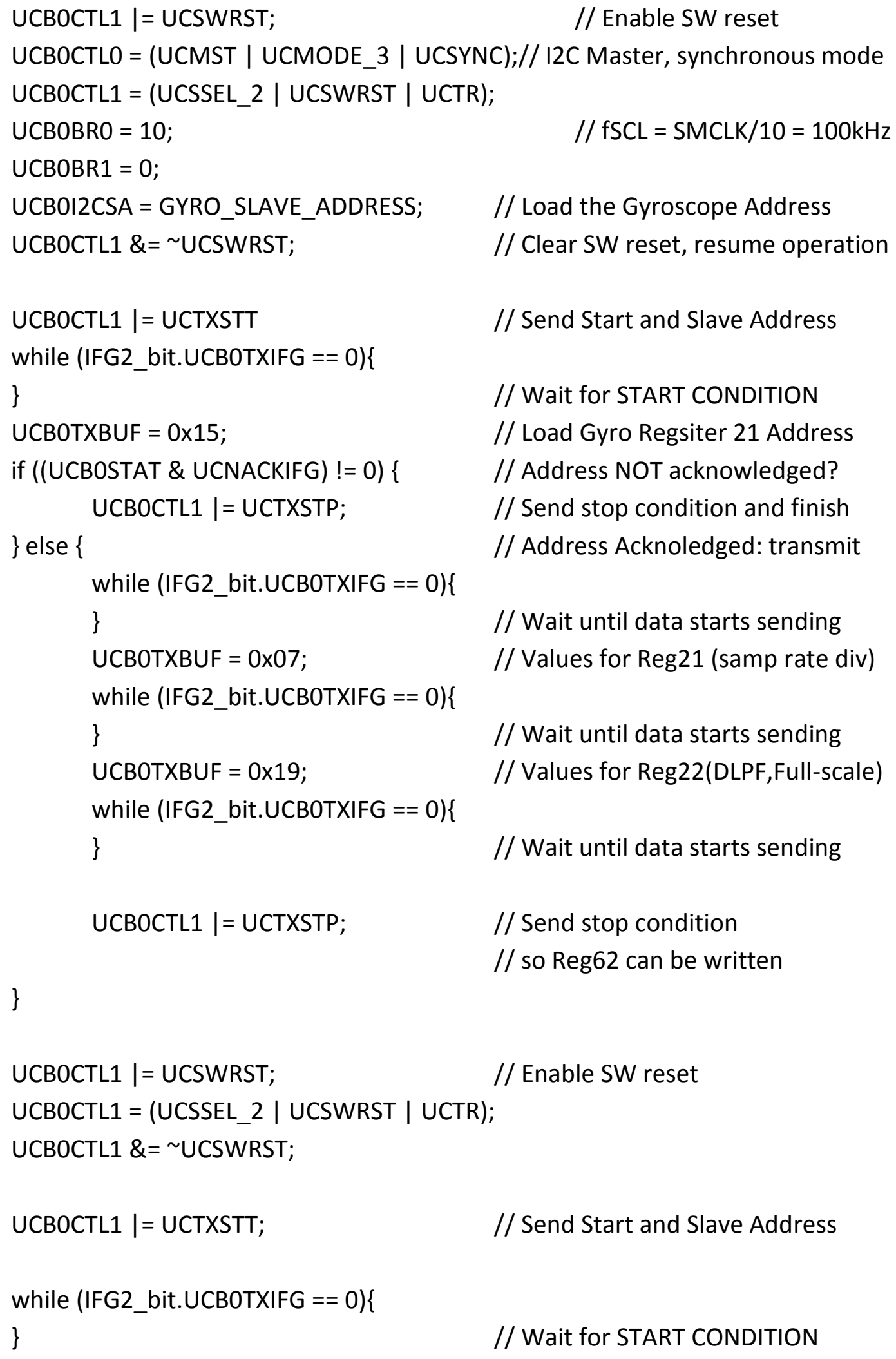




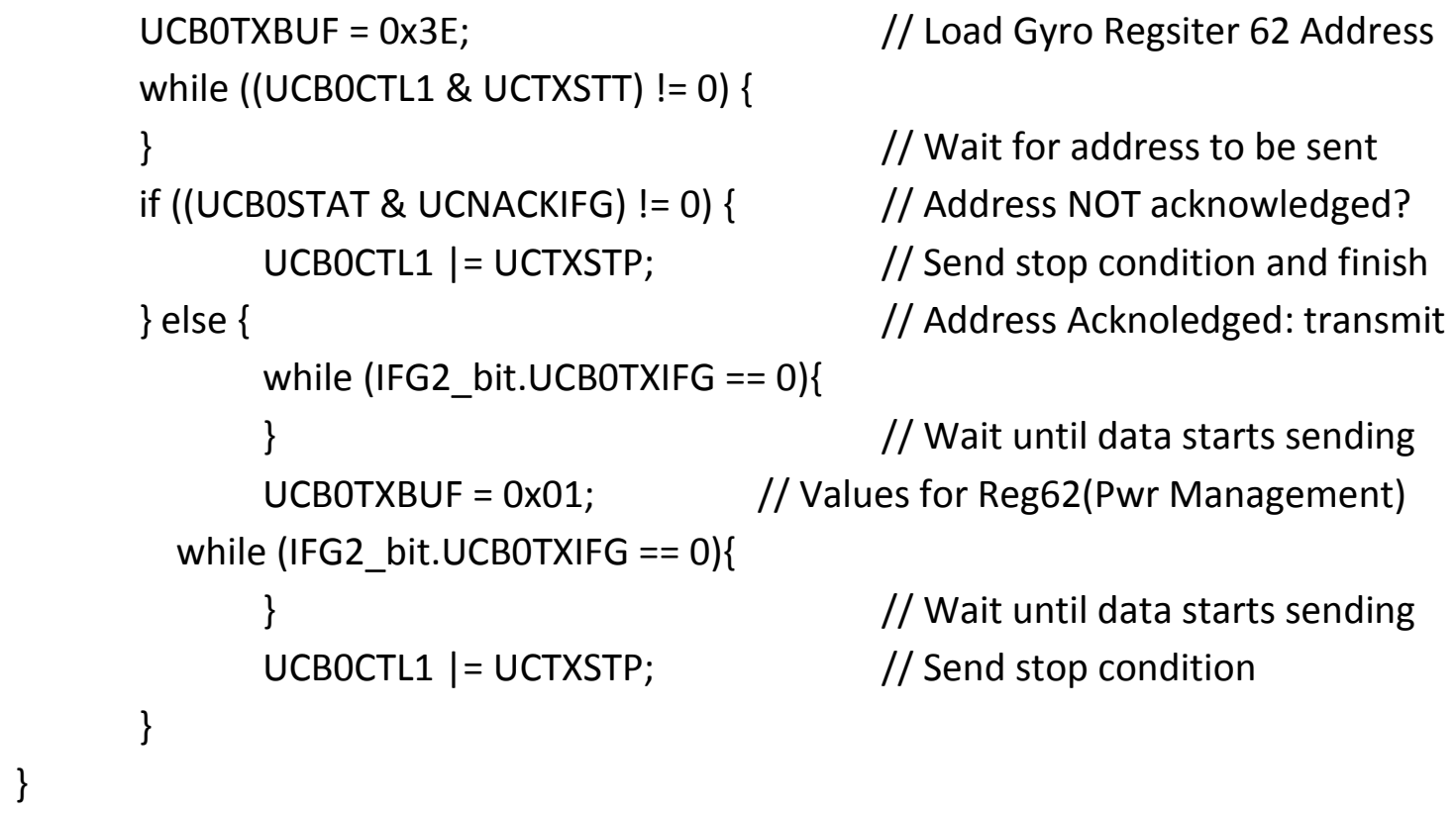


P2OUT_bit.P2OUT_3 =0;

// Set CE\# to low

while (! (IFG2 \& UCAOTXIFG) );

UCAOTXBUF = 0X01;

// Tx WRSR Command to Flash

for $(\mathrm{j}=10 ; \mathrm{j}>0$; $\mathrm{j}--)$;

while (! (IFG2 \& UCAORXIFG) );

UCAOTXBUF $=0 \times 00$;

// Set AAI mode and WriteEnable

for $(j=10 ; j>0 ; j--)$;

while (! (IFG2 \& UCAORXIFG));

P2OUT_bit.P2OUT_3 =1;

// Set CE\# high again

for $(j=100 ; j>0 ; j--)$;

// Perform a Write-Enable Operation before Chip-Erase

while (! (IFG2 \& UCAOTXIFG) );

// USCI_AO TX Buffer Ready?

P2OUT \& $=\sim 0 \times 08$;

// Set CE\# to low

UCAOTXBUF $=0 \times 06$;

// WREN Command to Flash

for $(\mathrm{j}=10 ; \mathrm{j}>0 ; \mathrm{j}-\mathrm{-})$;

while (! (IFG2 \& UCAORXIFG) );

P2OUT | = 0x08;

// Set CE\# high again

for $(\mathrm{j}=30 ; \mathrm{j}>0 ; \mathrm{j}--)$;

// Perform a Chip-Erase Command to prepare for AAI mode

while (! (IFG2 \& UCAOTXIFG) ); // USCI_A0 TX Buffer Ready?

P2OUT \& $=\sim 0 \times 08$;

// Set CE\# to low

UCAOTXBUF $=0 \times 60$;

// Chip Erase Command to Flash

for $(j=10 ; j>0 ; j--)$;

while (! (IFG2 \& UCAORXIFG) );

P2OUT $\mid=0 \times 08$;

// Set CE\# high again

for $(\mathrm{j}=30 ; \mathrm{j}>0$; $\mathrm{j}-\mathrm{-})$;

for $(j=10000 ; j>0 ; j--)$ // Wait $50 m s$ for chip erase

for $(j=10000 ; j>0 ; j--)$ // Wait $100 m$ for chip erase

// Perform a Write-Enable Operation before AAI

while (! (IFG2 \& UCAOTXIFG)); // USCI_AO TX Buffer Ready?

P2OUT \& $=\sim 0 \times 08$;

// Set CE\# to low

UCAOTXBUF = 0x06;

// WREN Command to Flash

for $(\mathrm{j}=10 ; \mathrm{j}>0 ; \mathrm{j}-\mathrm{-})$; 
while (! (IFG2 \& UCAORXIFG) );

P2OUT I=0x08; / / Set CE\# high again

for $(j=30 ; j>0 ; j--)$;

// Set up Auto Address Increment Program for Flash

while (! (IFG2 \& UCAOTXIFG) ); // USCl_A0 TX Buffer Ready?

P2OUT \&= 0X08; $\quad$ // Set CE\# to low

UCAOTXBUF = OXAF; / / AAI Command to Flash

for $(\mathrm{j}=10 ; \mathrm{j}>0 ; \mathrm{j}--)$;

while (! (IFG2 \& UCAORXIFG) );

while (! (IFG2 \& UCAOTXIFG) );

UCAOTXBUF $=0 \times 00$;

// A[23:16] Address to Flash

for $(j=10 ; j>0 ; j--)$;

while (! (IFG2 \& UCAORXIFG) );

while (! (IFG2 \& UCAOTXIFG) );

UCAOTXBUF = 0x00;

// A[15:8] Address to Flash

for $(j=10 ; j>0 ; j--)$;

while (! (IFG2 \& UCAORXIFG) );

while (! (IFG2 \& UCAOTXIFG));

UCAOTXBUF = 0X03;

// A[7:0] Address to Flash

for $(j=10 ; j>0 ; j--)$;

while (! (IFG2 \& UCAORXIFG) );

while (! (IFG2 \& UCAOTXIFG));

UCAOTXBUF = OXAA;

// Write Dummy Data to <000003>

for $(\mathrm{j}=10 ; \mathrm{j}>0$; $\mathrm{j}--)$;

while (! (IFG2 \& UCAORXIFG) );

P2OUT | = 0x08;

// Set CE\# high again

for $(\mathrm{j}=30 ; \mathrm{j}>0 ; \mathrm{j}--)$;

// Delay

\}

void MyApp_PutDummyDataToFlash( void )

\{

volatile unsigned int $\mathrm{i}$;

volatile unsigned int $\mathrm{d}$;

for $(d=15 ; d>0 ; d--)\{$ 
// Write Sensor Data to External Flash using Auto Address Increment (AAI) for $(\mathrm{i}=30$; $\mathrm{i}>0$; $\mathrm{i}-\mathrm{-})$; $\quad$ // Delay while (! (IFG2 \& UCAOTXIFG) ); // USCI_A0 TX Buffer Ready?

P2OUT \&= 0X08;

// Set CE\# to low

UCAOTXBUF = OXAF;

// AAI Command before data write

for ( $i=10$; $i>0$; $i--)$;

//while (! (IFG2 \& UCAORXIFG) );

//while (! (IFG2 \& UCAOTXIFG) );

UCAOTXBUF = d;

// Write Dummy data

//_no_operation();

for ( $i=10 ; i>0$; --$)$;

//while (! (IFG2 \& UCAORXIFG) );

P2OUT |= 0x08;

// Set CE\# high again

for $(i=10 ; i>0 ; i--)$;

// Delay

\}

//P2OUT \&= 0x08;

//UCAOTXBUF = 0X04;

//for ( $\mathrm{i}=15 ; \mathrm{i}>0$; $\mathrm{i}-\mathrm{-})$;

//P2OUT | = 0x08;

$/ /$ for $(\mathrm{i}=10$; $\mathrm{i}>0$; $\mathrm{i}--)$;

//P2OUT \& $=\sim 0 \times 08$;

//UCAOTXBUF = 0x05;

$/ /$ for $(\mathrm{i}=15 ; \mathrm{i}>0$; $\mathrm{i}-\mathrm{-})$;

//UCAOTXBUF = 0x00;

$/ /$ for $(\mathrm{i}=10$; $\mathrm{i}>0$; $\mathrm{i}--)$;

//P2OUT |= 0x08;
// Set CE\# to low

// Write Disable Command

// Set CE\# high again

// Delay

// Set CE\# to low

// Read status Register Command

// Delay

// TX junk to get Stat Reg values

// Delay

// Set CE\# high again

\}

void MyApp_GetSensorData( void )

\{

// Gather Accelerometer Data via I2C Interface

UCBOCTL1 |= UCSWRST; / / Enable SW reset 


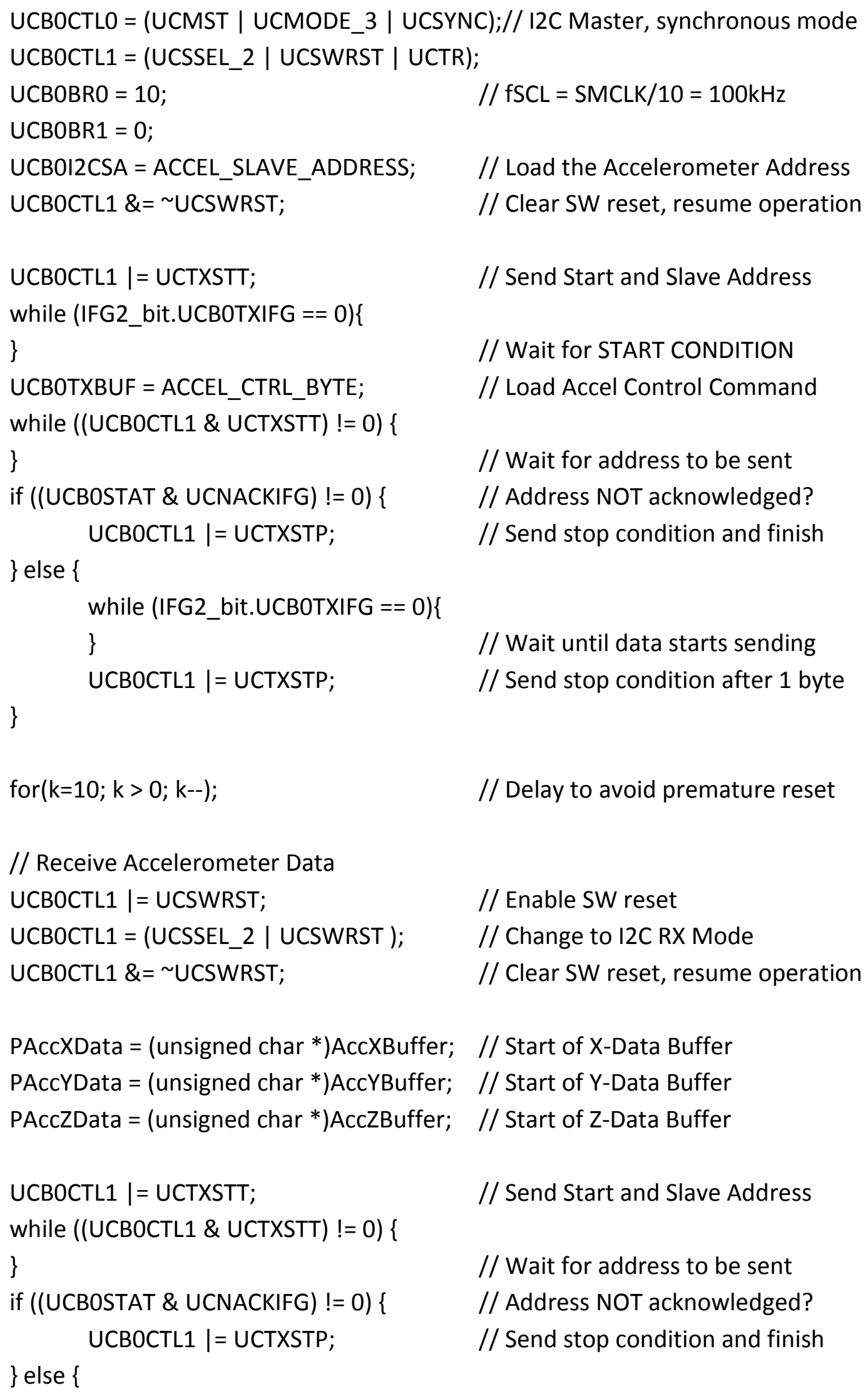

UCBOCTL1 I= UCTXSTT; $\quad$ // Send Start and Slave Address UCBOCTL1 |= UCTXSTP; $\quad$ // Send stop condition and finish

// Wait until data starts sending // Send stop condition after 1 byte

// Wait for address to be sent 


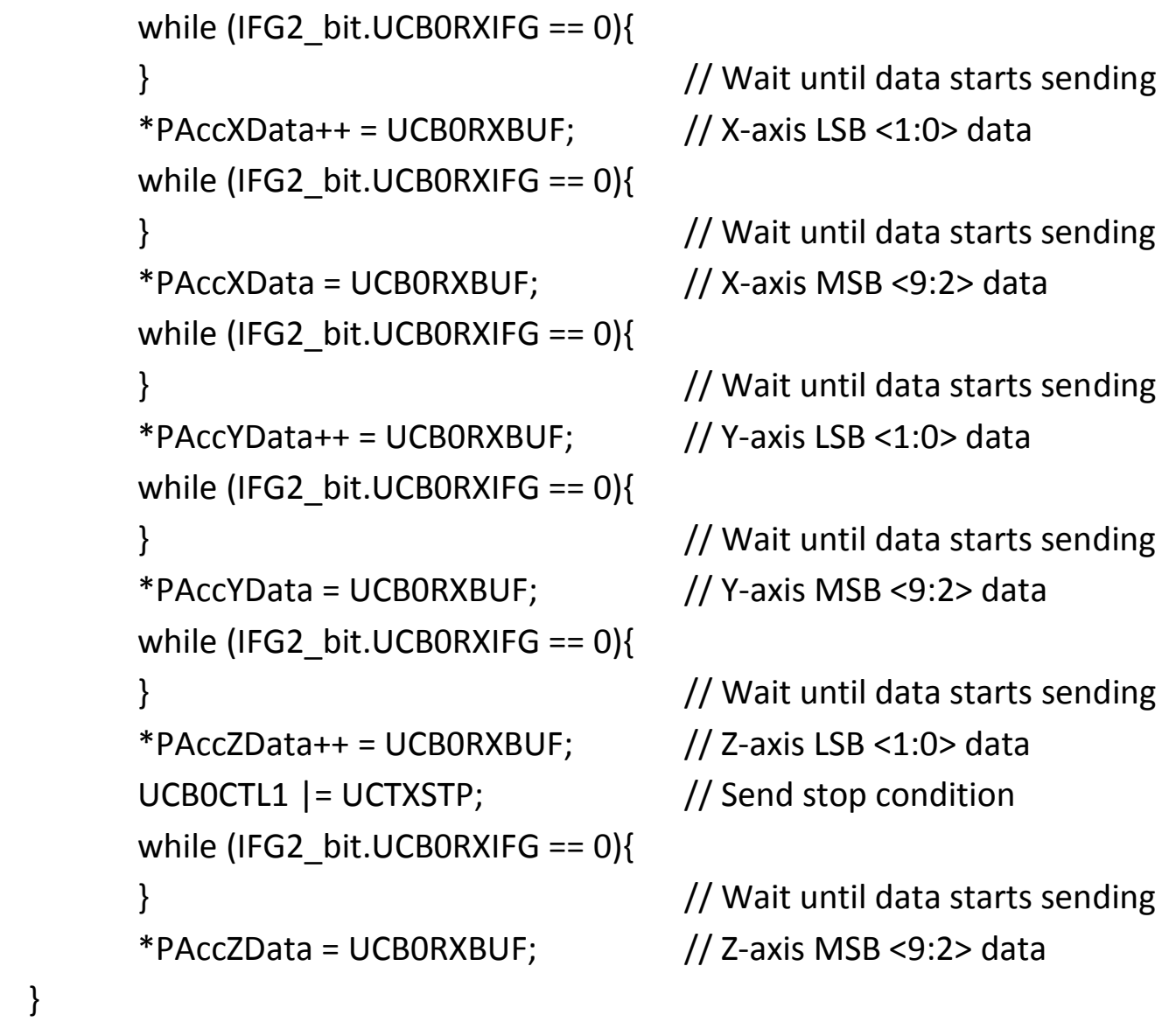

// Gather Gyroscope Data via I2C Interface UCBOCTL1 |= UCSWRST; / / Enable SW reset UCBOCTLO = (UCMST | UCMODE_3 | UCSYNC);// I2C Master, synchronous mode UCBOCTL1 = (UCSSEL_2 | UCSWRST | UCTR); UCBOI2CSA = GYRO_SLAVE_ADDRESS; // Load the Gyroscope Address UCBOCTL1 \& = UCSWRST; // Clear SW reset, resume operation

PGyrXData = (unsigned char *)GyrXBuffer; // Start of X-Data Buffer PGyrYData = (unsigned char *)GyrYBuffer; // Start of Y-Data Buffer PGyrZData = (unsigned char *)GyrZBuffer; // Start of Z-Data Buffer

UCBOCTL1 | = UCTXSTT;

// Send Start and Slave Address while (IFG2_bit.UCBOTXIFG $==0$ ) \{ \} // Wait for START CONDITION UCBOTXBUF = 0x1D; // Start of Gyro Data Address 
while ((UCBOCTL1 \& UCTXSTT) != 0) \{

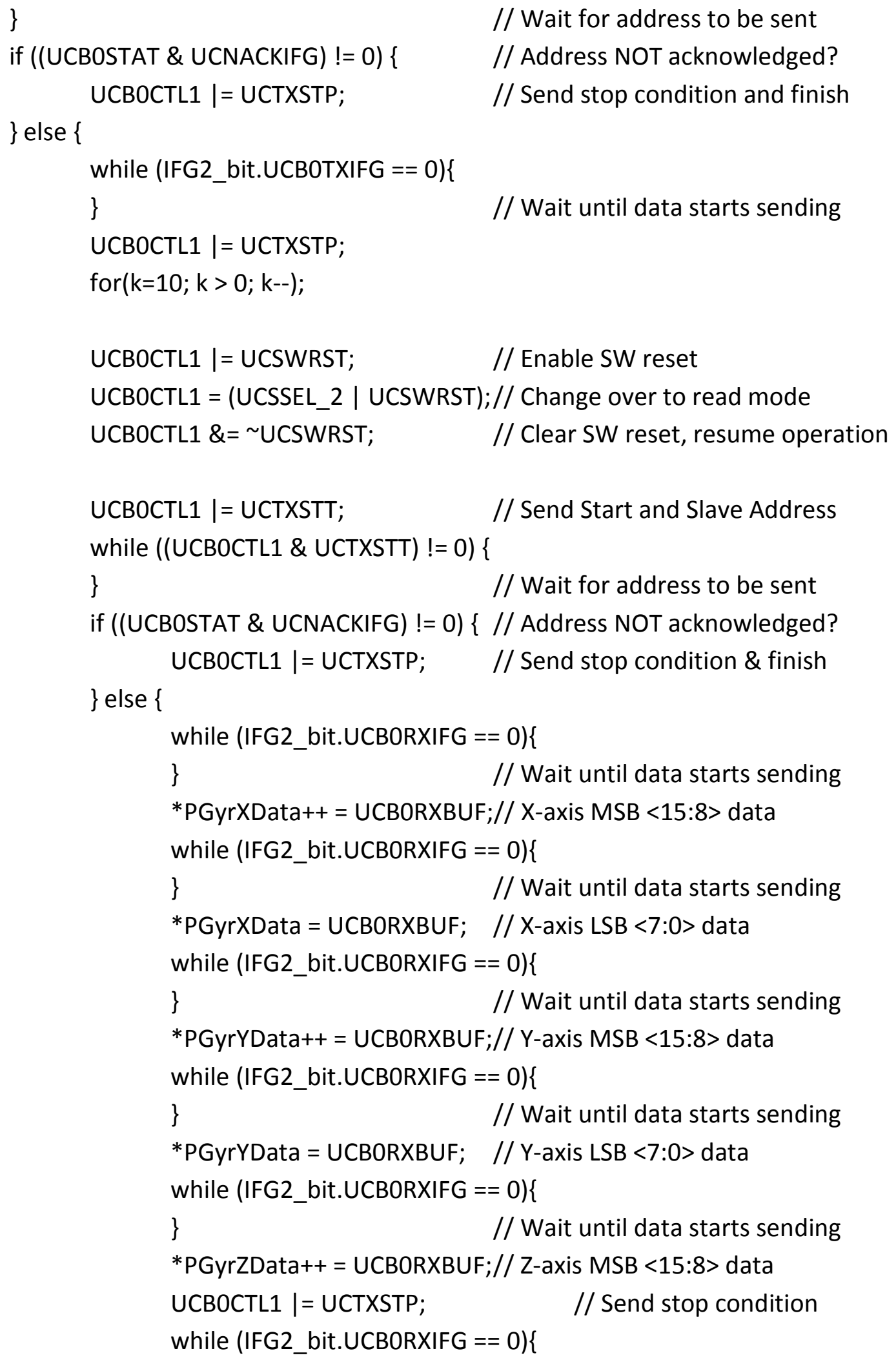




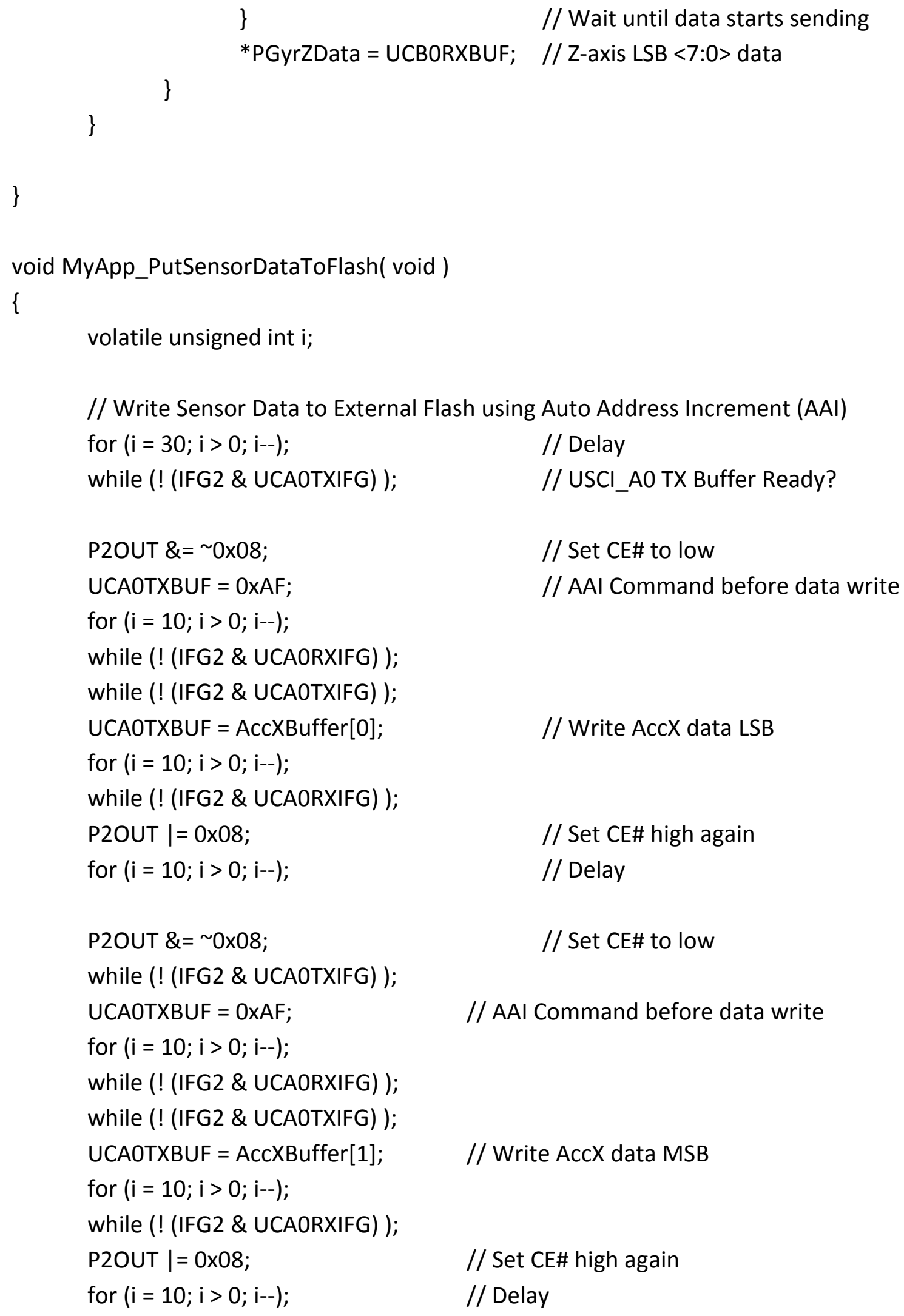


P2OUT \&= 0x08;

while (! (IFG2 \& UCAOTXIFG));

UCAOTXBUF = OXAF;

for $(i=10 ; i>0$; $i--)$;

while (! (IFG2 \& UCAORXIFG) );

while (! (IFG2 \& UCAOTXIFG));

UCAOTXBUF = AccYBuffer[0];

for $(i=10 ; i>0$; $i--)$;

while (! (IFG2 \& UCAORXIFG));

P2OUT I= 0x08;

for $(i=10$; $>0$; $i--)$;

P2OUT \&= 0x08;

while (! (IFG2 \& UCAOTXIFG) );

UCAOTXBUF = OXAF;

for ( $i=10$; $>$ > $i--)$;

while (! (IFG2 \& UCAORXIFG) );

while (! (IFG2 \& UCAOTXIFG) );

UCAOTXBUF = AccYBuffer[1];

for $(i=10 ; i>0 ; i--)$;

while (! (IFG2 \& UCAORXIFG));

P2OUT |= 0x08;

for $(i=10$; $i>0$; --$)$;

P2OUT \&= 0x08;

while (! (IFG2 \& UCAOTXIFG));

UCAOTXBUF = OXAF;

for ( $i=10$; $>$ > ; $i--)$;

while (! (IFG2 \& UCAORXIFG));

while (! (IFG2 \& UCAOTXIFG));

UCAOTXBUF = AccZBuffer[0];

for ( $i=10$; $>$ > ; $i--)$;

while (! (IFG2 \& UCAORXIFG));

P2OUT I= 0x08;

for $(i=10$; $i>0$; --$)$;

P2OUT \&= 0x08;
// Set CE\# to low

// AAI Command before data write

// Write AccY data LSB

// Set CE\# high again

// Delay

// Set CE\# to low

// AAI Command before data write

// Write AccY data MSB

// Set CE\# high again

// Delay

// Set CE\# to low

// AAI Command before data write

// Write AccZ data LSB

// Set CE\# high again

// Delay

// Set CE\# to low 
while (! (IFG2 \& UCAOTXIFG));

UCAOTXBUF = OXAF;

// AAI Command before data write

for $(i=10$; $>0$; $i--)$;

while (! (IFG2 \& UCAORXIFG));

while (! (IFG2 \& UCAOTXIFG) );

UCAOTXBUF = AccZBuffer[1];

// Write AccZ data MSB

for ( $\mathrm{i}=10$; $\mathrm{i}>0$; $\mathrm{i}-$ );

while (! (IFG2 \& UCAORXIFG) );

P2OUT |= 0x08;

// Set CE\# high again

for $(i=10 ; i>0$; --$)$;

// Delay

// Write Gyro Data to Memory

P2OUT \& = 0x08;

// Set CE\# to low

while (! (IFG2 \& UCAOTXIFG));

UCAOTXBUF = OXAF;

// AAI Command before data write

for $(i=10 ; i>0$; $i--)$;

while (! (IFG2 \& UCAORXIFG) );

while (! (IFG2 \& UCAOTXIFG));

UCAOTXBUF = GyrXBuffer[0];

// Write GyrX data MSB

for ( $i=10 ; i>0$; --$)$;

while (! (IFG2 \& UCAORXIFG));

P2OUT |=0x08;

// Set CE\# high again

for $(i=10 ; i>0$; --$)$;

// Delay

P2OUT \&= 0x08;

// Set CE\# to low

while (! (IFG2 \& UCAOTXIFG) );

UCAOTXBUF = OXAF;

// AAI Command before data write

for $(i=10$; $>0$; $i--)$;

while (! (IFG2 \& UCAORXIFG));

while (! (IFG2 \& UCAOTXIFG) );

UCAOTXBUF = GyrXBuffer[1];

// Write GyrX data LSB

for ( $i=10 ; i>0$; -- );

while (! (IFG2 \& UCAORXIFG) );

P2OUT |= 0x08;

// Set CE\# high again

for ( $i=10$; $>$ > $i--)$;

// Delay

P2OUT \&= 0x08;

// Set CE\# to low 
while (! (IFG2 \& UCAOTXIFG));

UCAOTXBUF $=0 \times A F$

// AAI Command before data write

for $(i=10$; $>0$; $i--)$;

while (! (IFG2 \& UCAORXIFG) );

while (! (IFG2 \& UCAOTXIFG) );

UCAOTXBUF = GyrYBuffer[0];

// Write GyrY data MSB

for ( $\mathrm{i}=10$; $\mathrm{i}>0$; $\mathrm{i}-$ );

while (! (IFG2 \& UCAORXIFG) );

P2OUT |= 0x08;

// Set CE\# high again

for $(i=10 ; i>0$; --$)$;

// Delay

P2OUT \&= 0x08;

// Set CE\# to low

while (! (IFG2 \& UCAOTXIFG));

UCAOTXBUF = OXAF;

// AAI Command before data write

for $(i=10 ; i>0$; $i--)$;

while (! (IFG2 \& UCAORXIFG) );

while (! (IFG2 \& UCAOTXIFG) );

UCAOTXBUF = GyrYBuffer[1];

// Write GyrY data LSB

for ( $i=10$; $>$ > ; $i--)$;

while (! (IFG2 \& UCAORXIFG) );

P2OUT I= 0x08;

for ( $i=10$; $i>0$; - );

// Set CE\# high again

// Delay

P2OUT \&= 0x08;

// Set CE\# to low

while (! (IFG2 \& UCAOTXIFG));

UCAOTXBUF = OXAF;

// AAI Command before data write

for ( $\mathrm{i}=10$; $\mathrm{i}>0$; $\mathrm{i}--)$;

while (! (IFG2 \& UCAORXIFG));

while (! (IFG2 \& UCAOTXIFG));

UCAOTXBUF = GyrZBuffer[0];

// Write GyrZ data MSB

for $(i=10 ; i>0$; --$)$;

while (! (IFG2 \& UCAORXIFG));

P2OUT |= 0x08;

// Set CE\# high again

for $(i=10 ; i>0$; --$)$;

// Delay

P2OUT \&= 0x08;

// Set CE\# to low

while (! (IFG2 \& UCAOTXIFG) );

UCAOTXBUF = OXAF;

// AAl Command before data write 
for $(\mathrm{i}=10 ; \mathrm{i}>0 \text {; } \mathrm{i}-)_{\text {; }}$

while (! (IFG2 \& UCAORXIFG) );

while (! (IFG2 \& UCAOTXIFG));

UCAOTXBUF = GyrZBuffer[1]; // Write GyrZ data LSB

for ( $\mathrm{i}=10 ; \mathrm{i}>0$; $\mathrm{i}-$ ) ;

while (! (IFG2 \& UCAORXIFG) );

P2OUT $\mid=0 \times 08$;

// Set CE\# high again

for ( $i=10 ; i>0$; --$)$;

// Delay

\} 


\section{Appendix C}

\section{C-language Program for RS-232 Data Transmission}

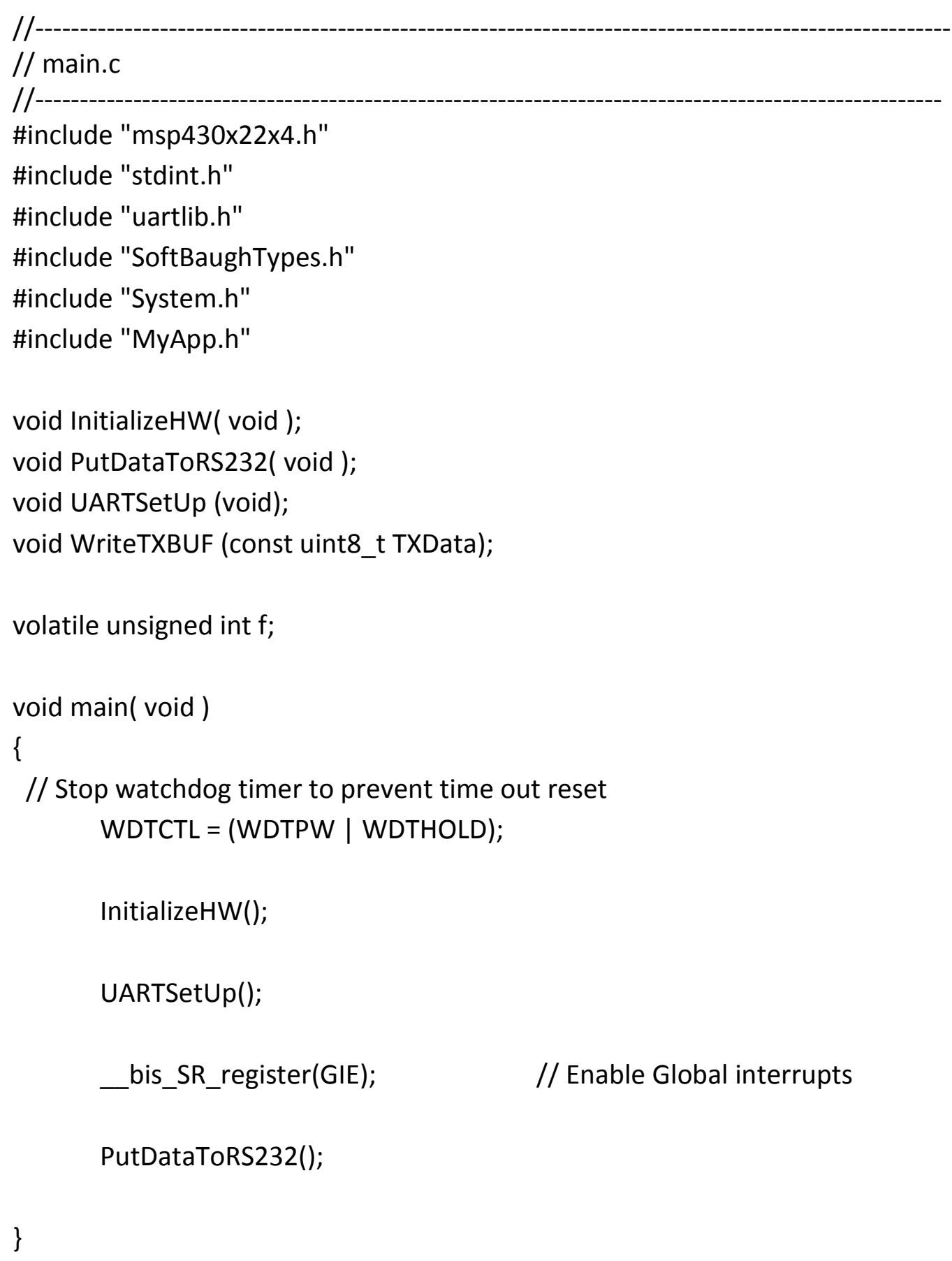




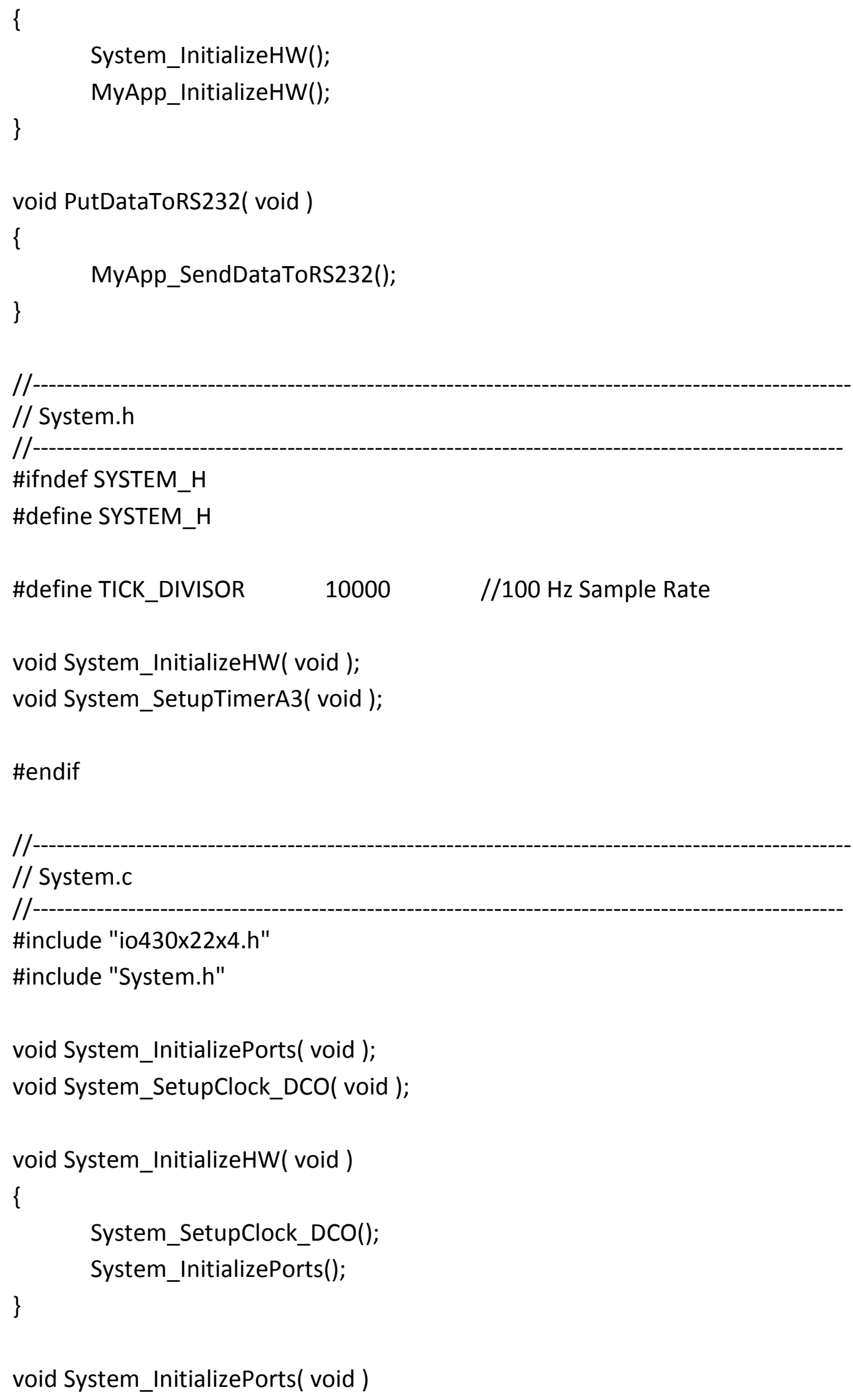


// Port1 initialization

// P1.0 - n/c

// P1.1 - n/c

// P1.2 - n/c or RS-232(TX)

// P1.3 $-\mathrm{n} / \mathrm{c}$ or RS-232(RX)

// P1.4 - n/c

// P1.5 - n/c

// P1.6 - n/c

// P1.7 - n/c

P1OUT $=(0 \times 04)$;

$\mathrm{P} 1 \mathrm{DIR}=(0 \mathrm{xF} 7)$;

$\mathrm{P} 1 \mathrm{SEL}=(0 \times 0 \mathrm{C})$;

// Port2 initialization

// P2.0 - n/c

// P2.1 - n/c

// P2.2 - Chip Enable /CE (SPI Flash Memory Enable)

// P2.3 - Connect to Flash \#HD (Hold)

// P2.4 - n/c

// P2.5-n/c

// P2.6-n/c

// P2.7 - n/c

P2OUT = (0x0C);

$\mathrm{P} 2 \mathrm{DIR}=(0 \mathrm{xFF})$;

$\mathrm{P} 2 \mathrm{SEL}=(0 \times 00)$;

// Port3 initialization

// P3.0 - USCI_A0_CLK (SPI for Ext. Flash Memory)

// P3.1 - I2C SDA

// P3.2 - 12C SCL

// P3.3 - n/c

// P3.4 - Data Out (SPI USCI_AO_SIMO)

// P3.5 - Data In (SPI USCI_A0_SOMI)

// P3.6 - n/c

// P3.7 - Connect to Flash \#WP (Write Protect)

P3OUT $=(0 \times 80)$; 


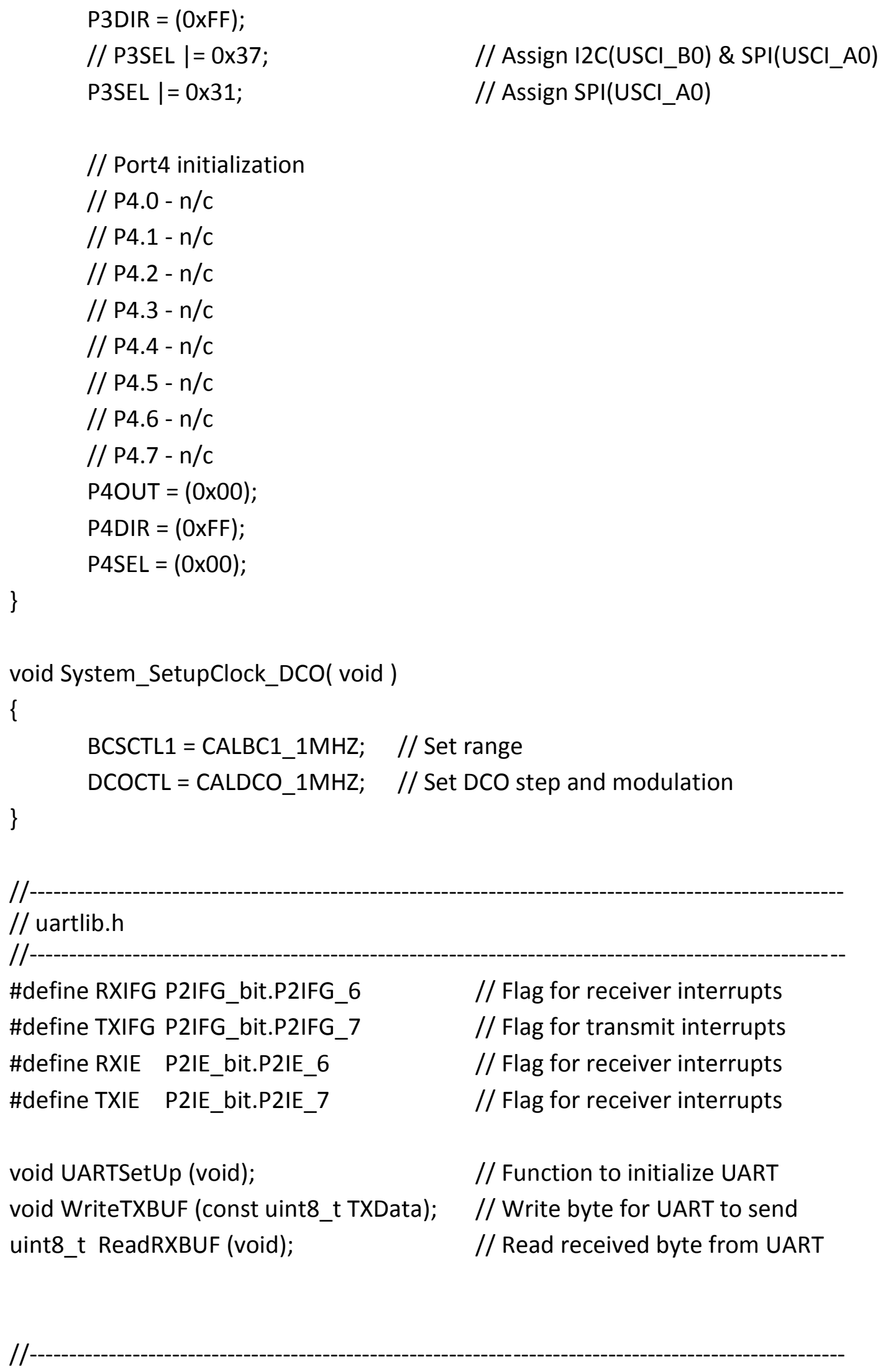




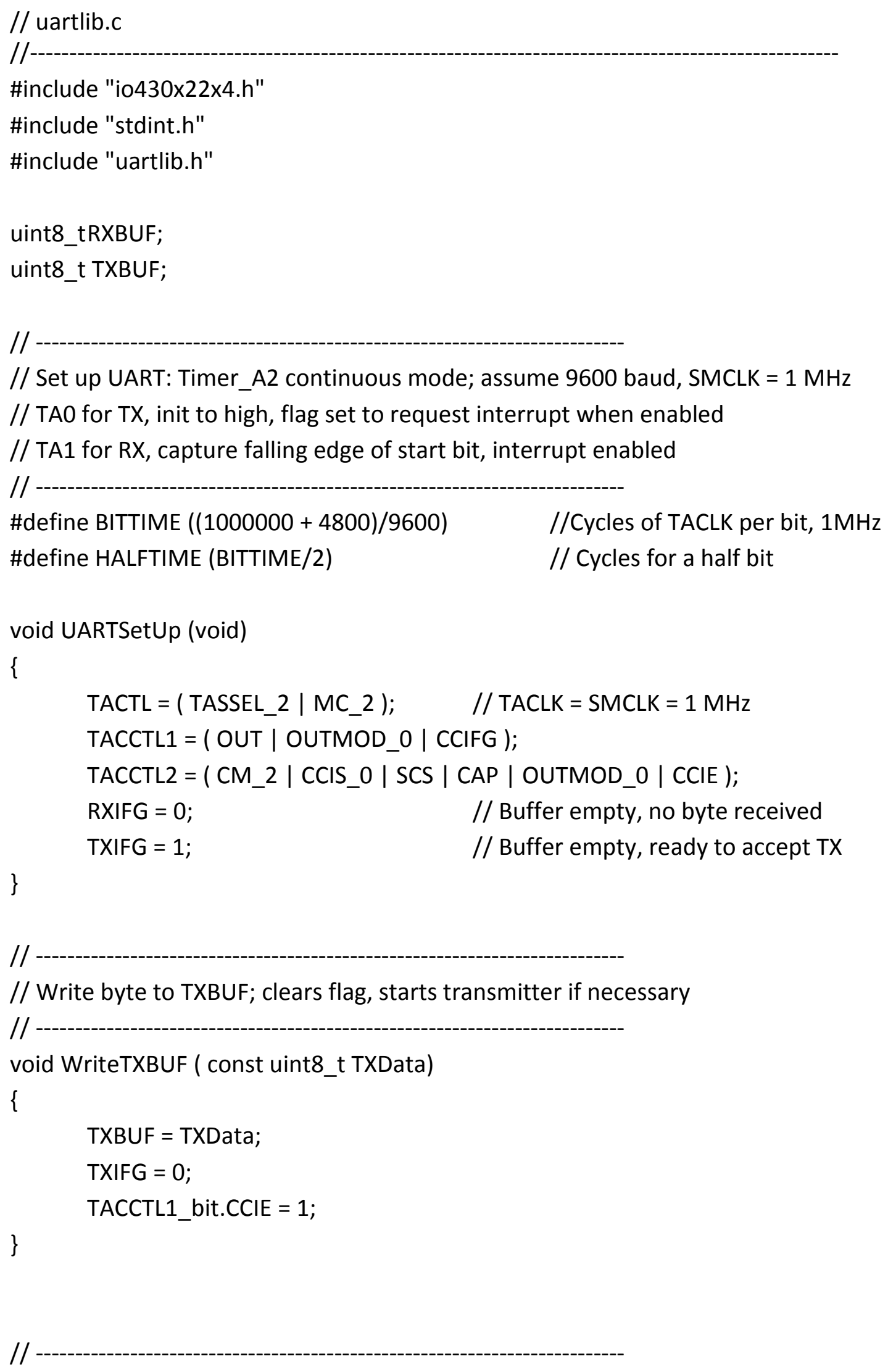


// Read byte from RXBUF; clears flag

//

uint8_t ReadRXBUF (void)

\{

RXIFG = 0;

return RXBUF;

\}

// -----------------------------------------------------------

// ISR for Timer_A3, channel 1: transmission

// ---------------------------------------------------

\#pragma vector $=$ TIMERA1_VECTOR

_interrupt void TIMERA1_ISR (void)

\{

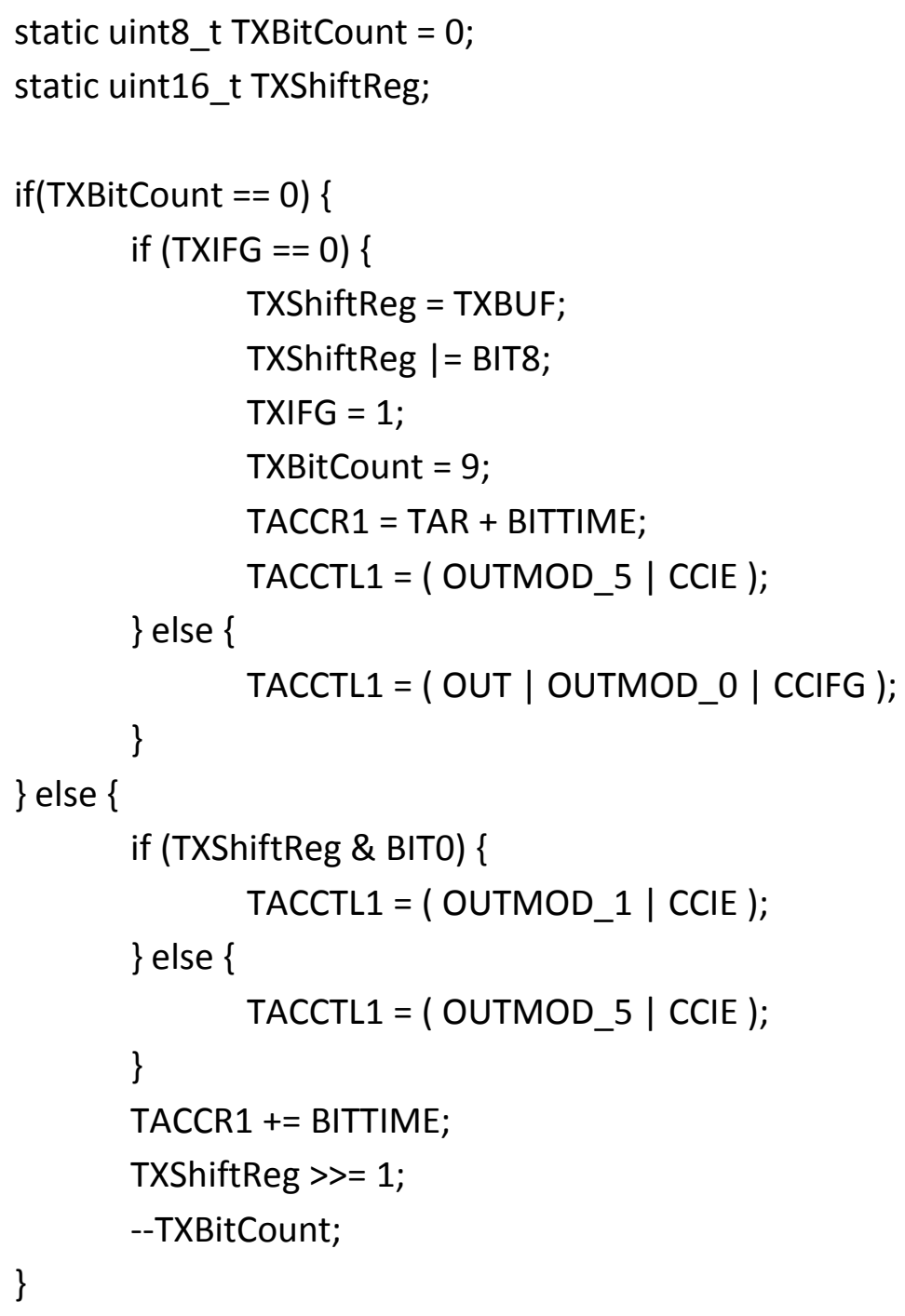




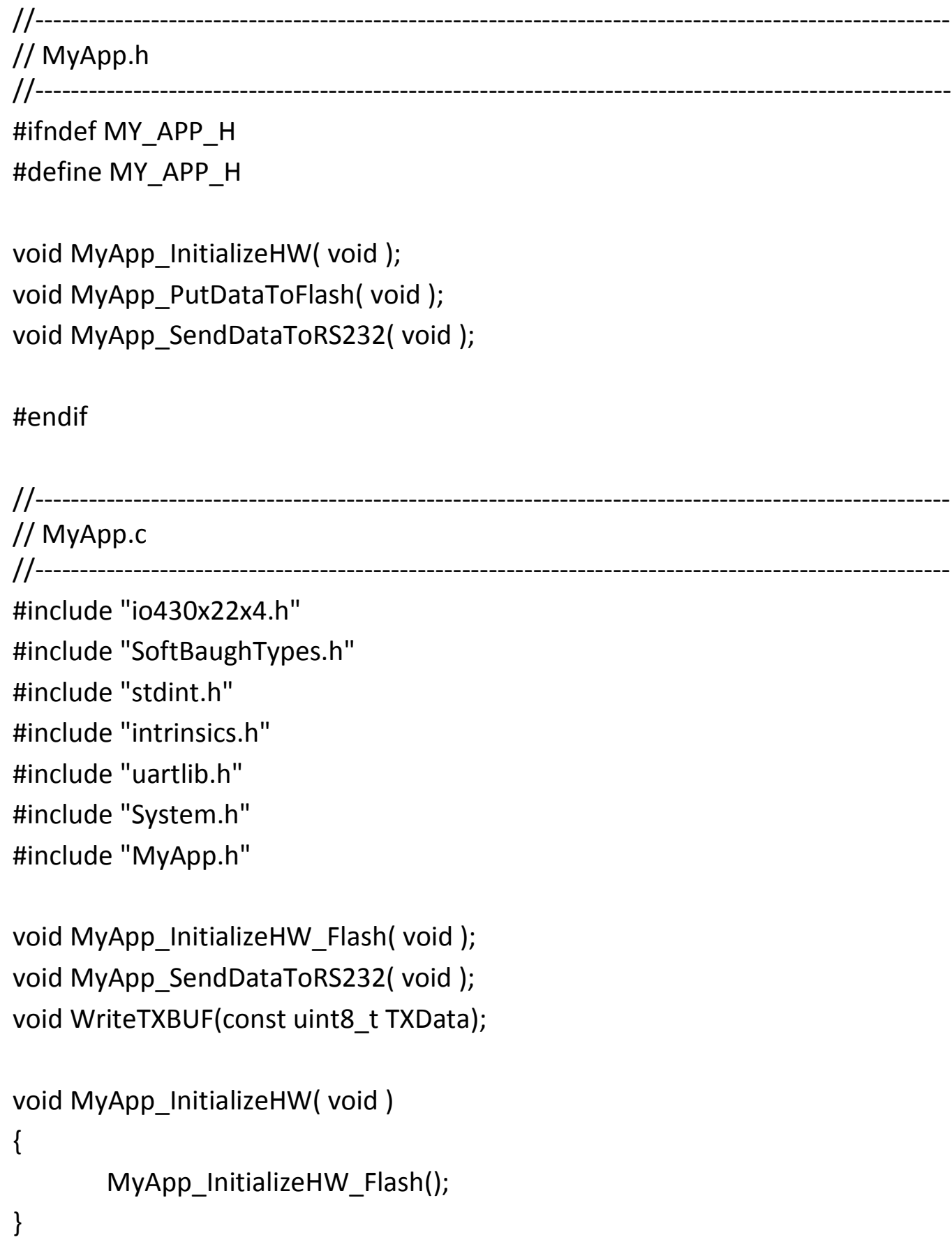


volatile unsigned int $\mathrm{j}$;

// Initialize External Flash via SPI Interface

UCA0CTL1 | = UCSWRST;

UCAOCTLO = ( UCCKPL | UCMSB | UCMST | UCMODE_0 | UCSYNC);

UCAOCTL1 | = (UCSSEL_2);

// SMCLK

$\mathrm{UCAOBRO}=10$

$/ / \mathrm{fSPI}=1 \mathrm{MHz} / 10=100 \mathrm{kHz}$

UCAOBR1 = 0;

$\mathrm{UCAOMCTL}=0$;

//Clear Modulation Control Bit

UCAOCTL1 \&= UCSWRST;

// Perform a EWSR \& WRSR to configure the Flash Status Register

while (! (IFG2 \& UCAOTXIFG));

P2OUT_bit.P2OUT_3 =0;

UCAOTXBUF = 0x50;

for $(j=10 ; j>0 ; j--)$;

while (! (IFG2 \& UCAORXIFG));

P2OUT_bit.P2OUT_3 =1;

for ( $\mathrm{j}=30 ; \mathrm{j}>0$; $\mathrm{j}--)$;

P2OUT_bit.P2OUT_3 = 0;

while (! (IFG2 \& UCAOTXIFG));

UCAOTXBUF = 0x01;

for $(j=10 ; j>0 ; j--)$;

while (! (IFG2 \& UCAORXIFG) );

UCAOTXBUF = 0x00;

for $(j=10 ; j>0 ; j--)$;

while (! (IFG2 \& UCAORXIFG));

P2OUT_bit.P2OUT_3 =1;

for $(j=100 ; j>0 ; j--)$;
// USCI_AO TX Buffer Ready?

// Set CE\# to low

// Tx EWSR Command to Flash

// Set CE\# high again

// Set CE\# to low

// Tx WRSR Command to Flash

// Set AAI mode and WriteEnable

// Set CE\# high again

\}

void MyApp_SendDataToRS232( void ) 


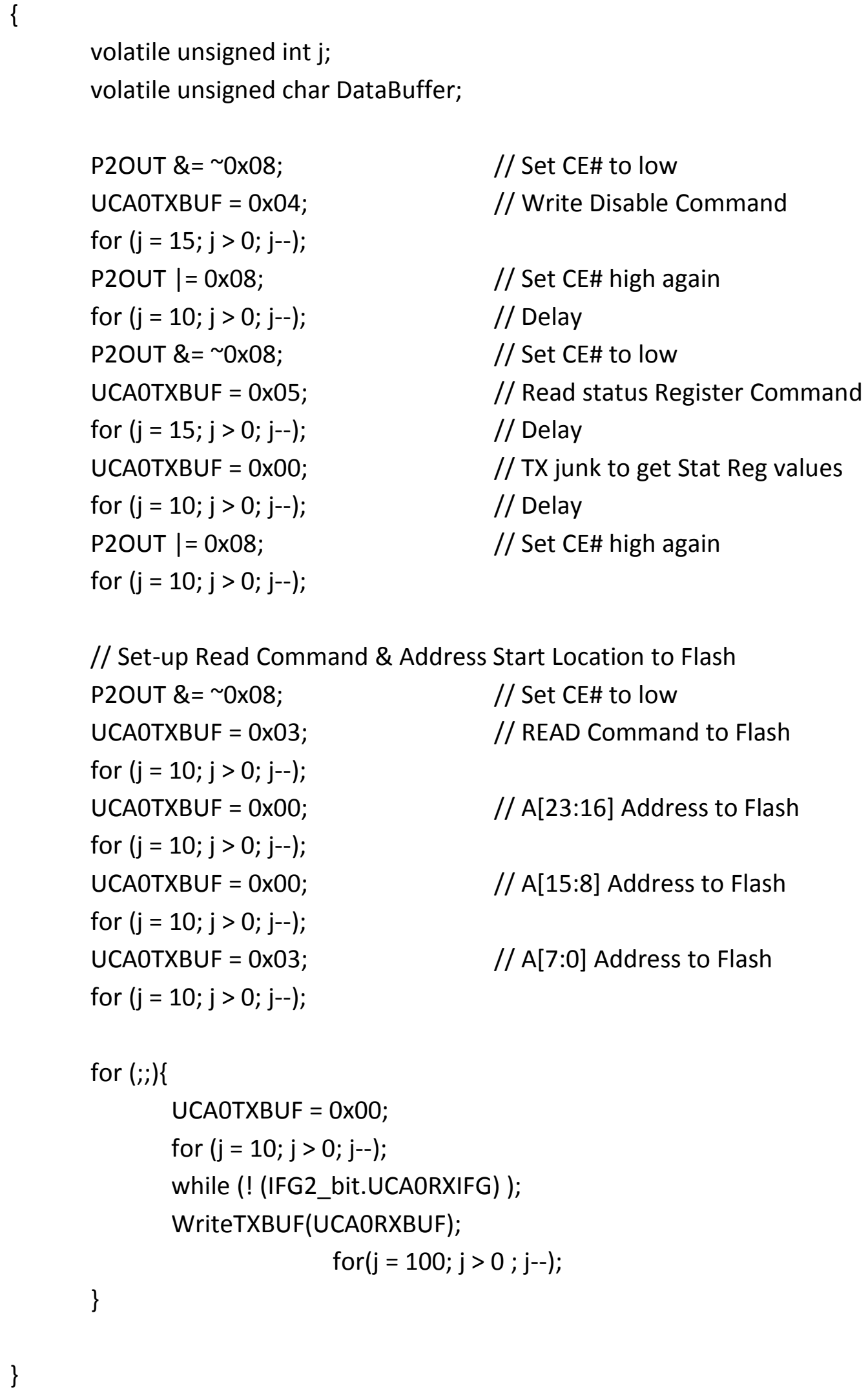




\section{Appendix D}

\section{Perl Language Program for Sensor Data Conversion}

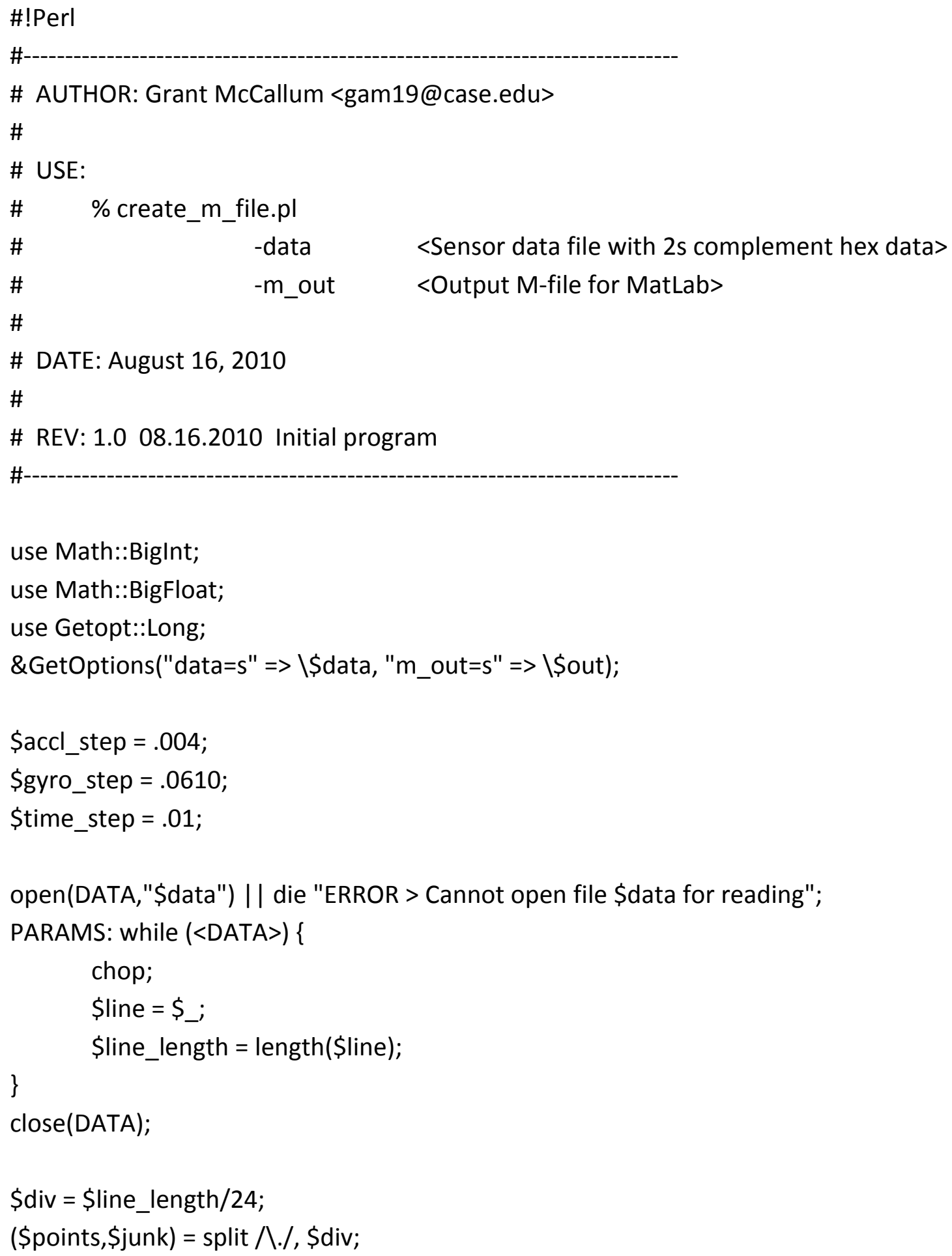




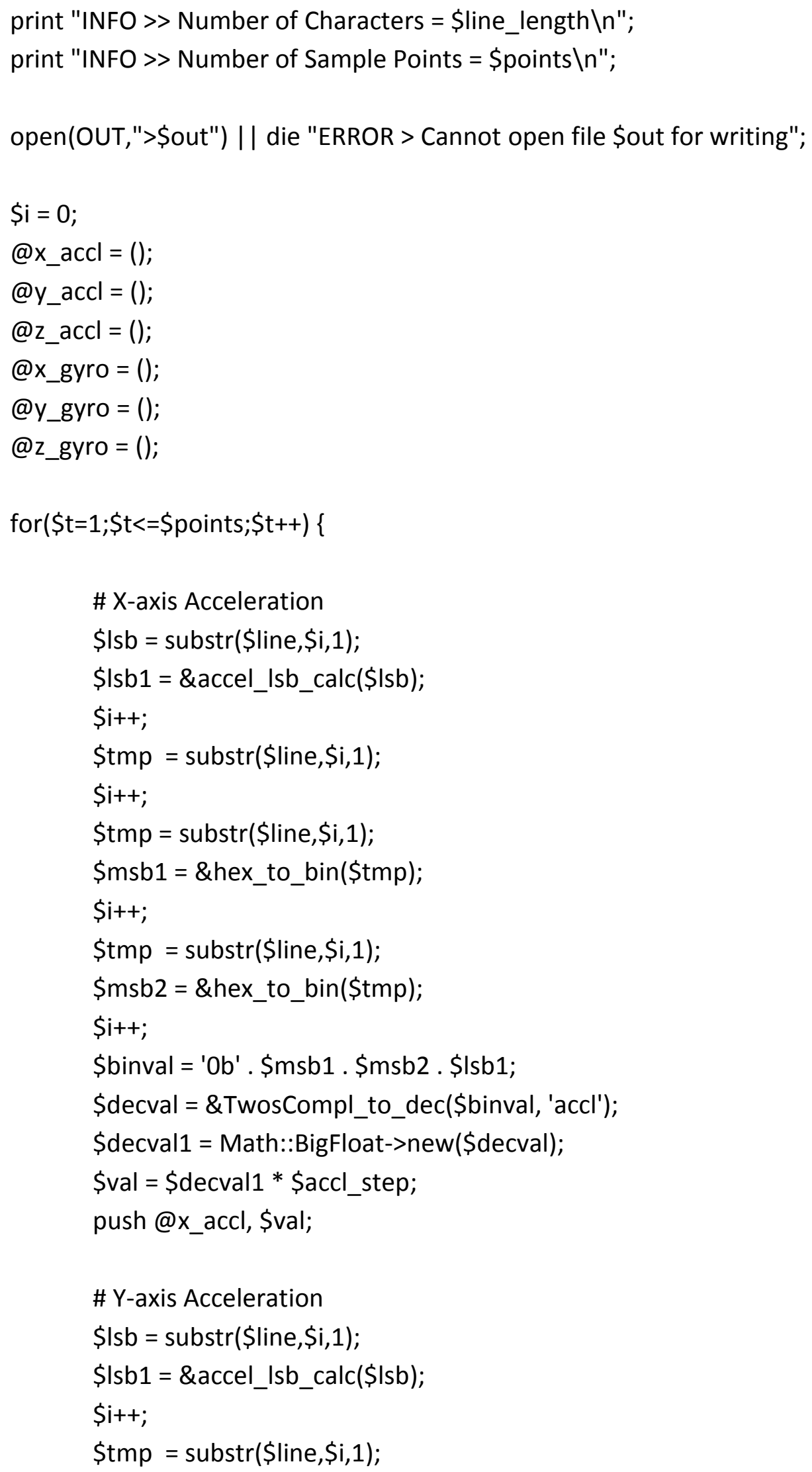




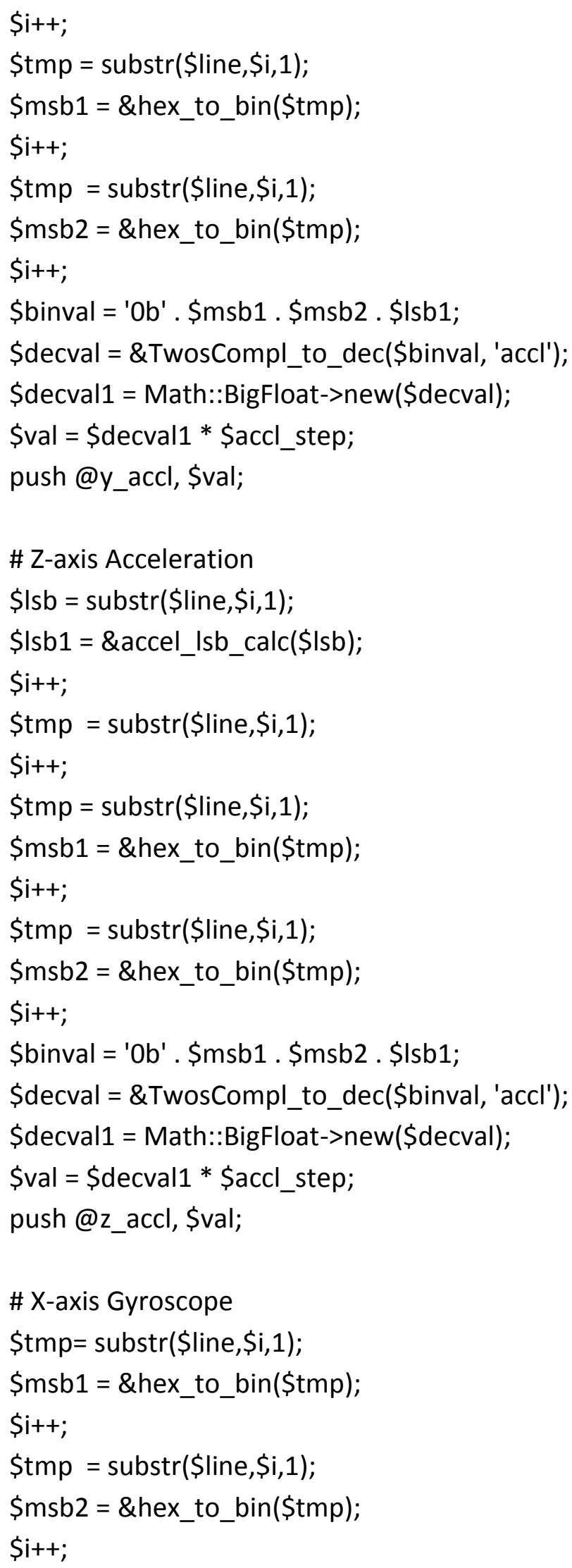




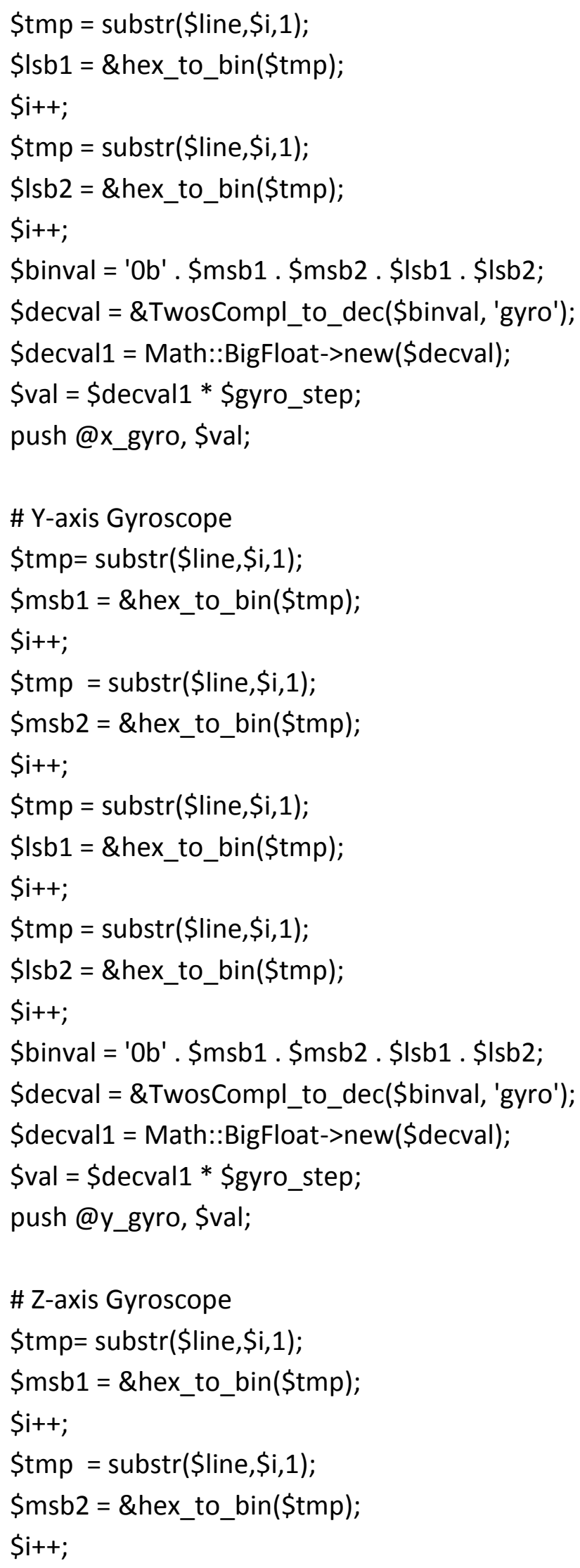




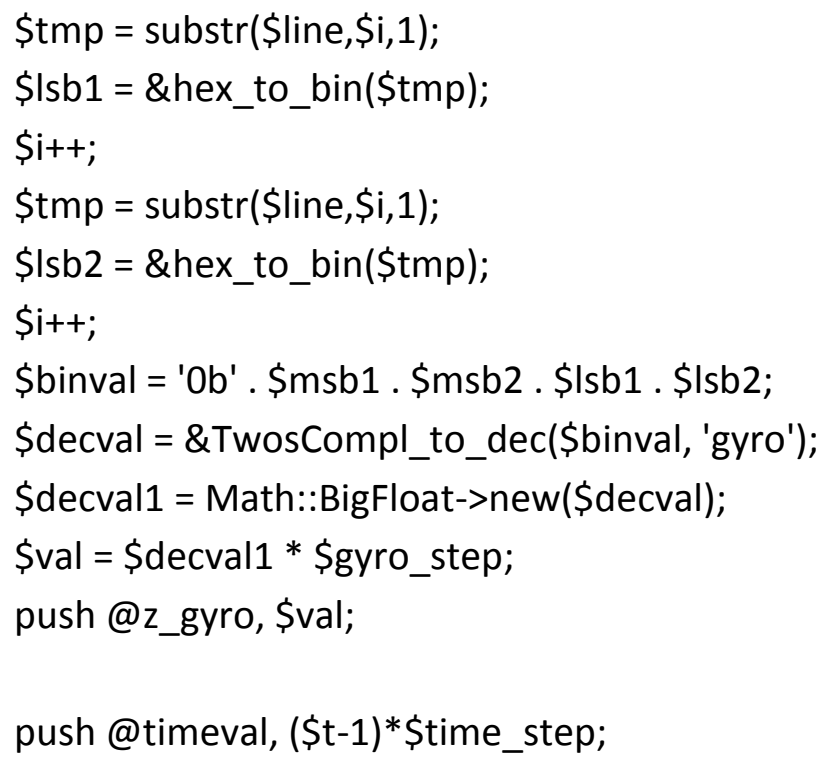


print OUT "y_gyro = [ \$y_gyro_data ] $\backslash \backslash \backslash n \backslash n " ;$

print OUT "\\% Z-AXIS Gyroscope Data\n";

\$z_gyro_data = join " ", @z_gyro;

print OUT "z_gyro = [ \$z_gyro_data ] ; $\backslash n \backslash n " ;$

print OUT "\\% Time Point Vector\n";

\$time_data = join " ", @timeval;

print OUT "timept = [ \$time_data ] $\backslash ; \backslash n \backslash n " ;$

print OUT "\\% Generate Acceleration Plots $\backslash n$ ";

print OUT "plot(timept,x_accl)\n";

print OUT "hold on $\backslash ; \backslash n$ ";

print OUT "plot(timept,y_accl, $\backslash$ 'g $g$ ') $\backslash n$ ";

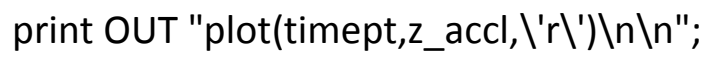

print OUT "\\% Generate Gyroscope Plots \n";

print OUT "plot(timept,x_gyro) \n";

print OUT "hold on \; \n";

print OUT "plot(timept,y_gyro, \'g\')\n";

print OUT "plot(timept,z_gyro, $\left.\backslash^{\prime} r \backslash^{\prime}\right) \backslash n \backslash n "$;

close(OUT);

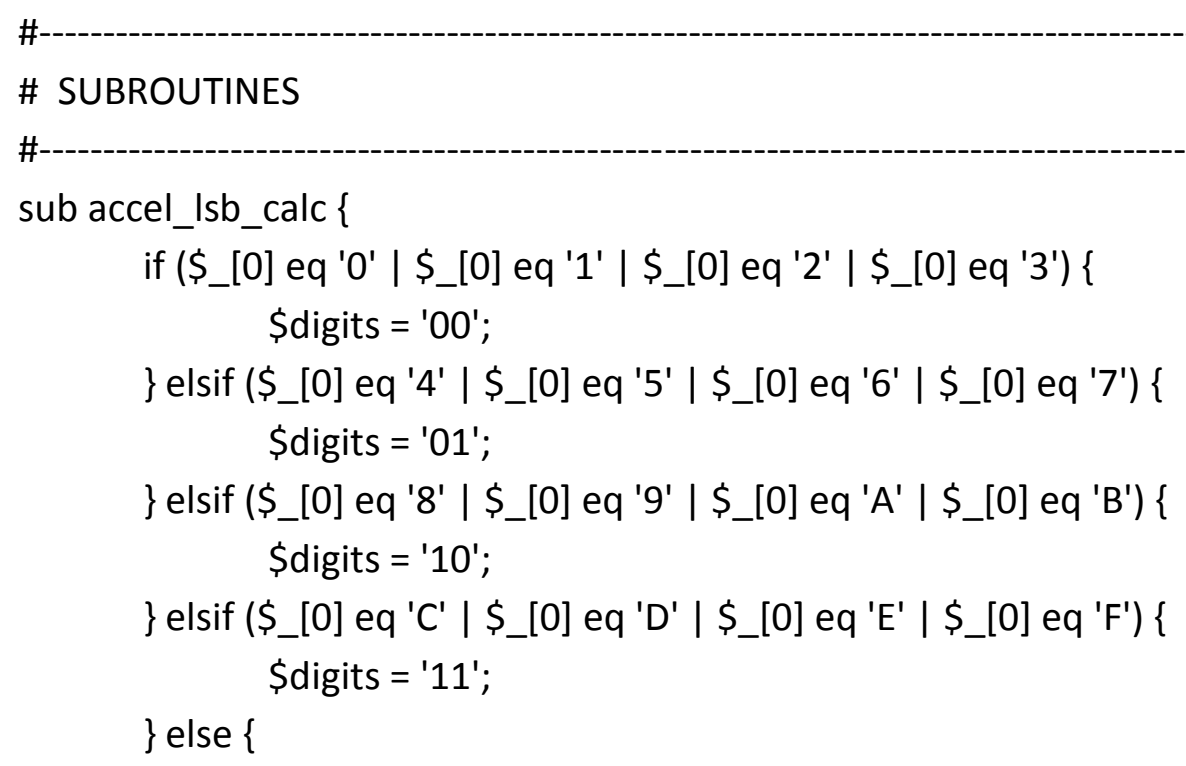


die "\n\nERROR >> Subroutine accel_Isb_calc found unknown data value for bits $<1: 0>-->$ \$_[0]\n\n";

\}

\$digits;

\}

sub TwosCompl_to_dec \{

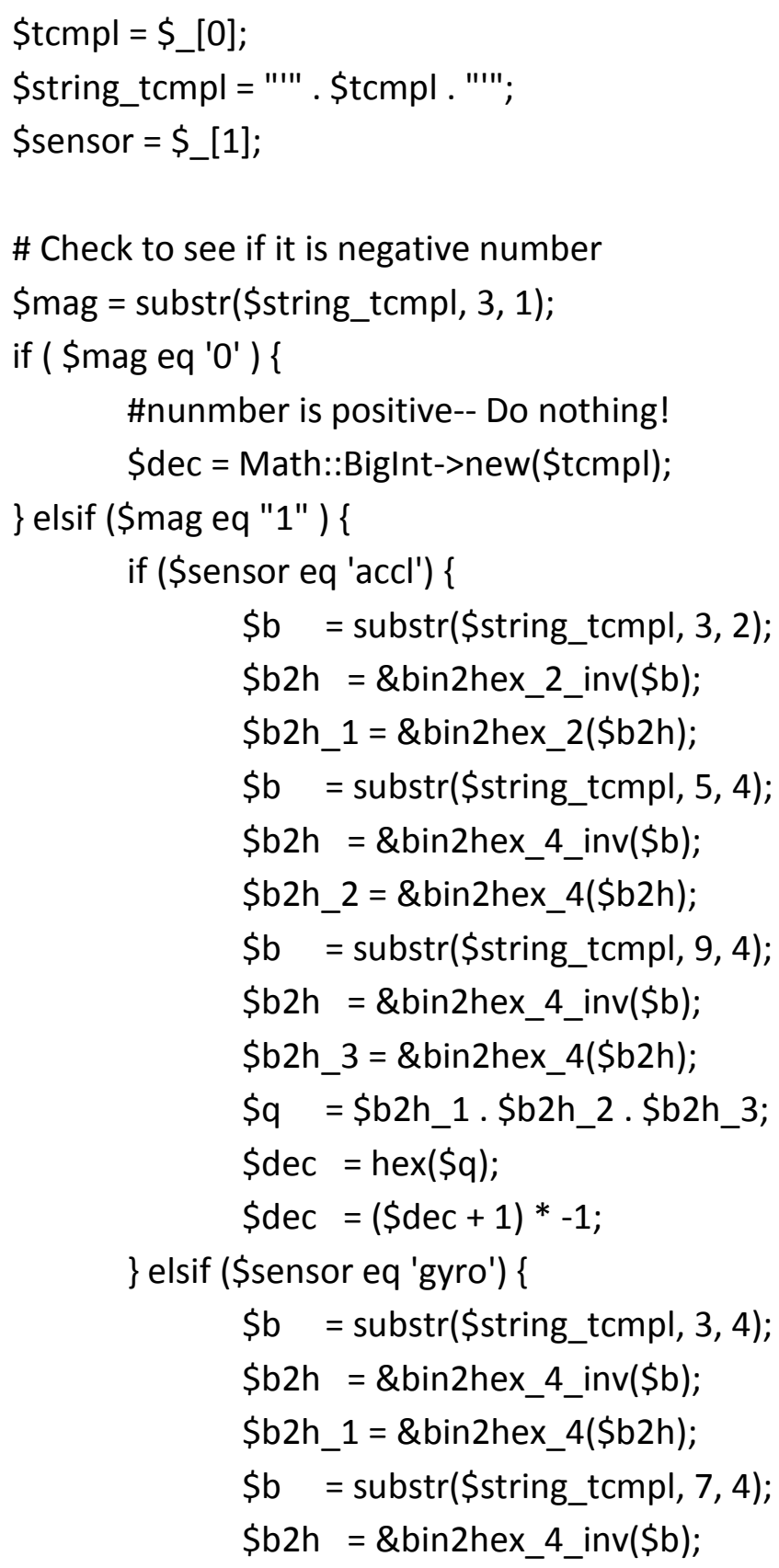




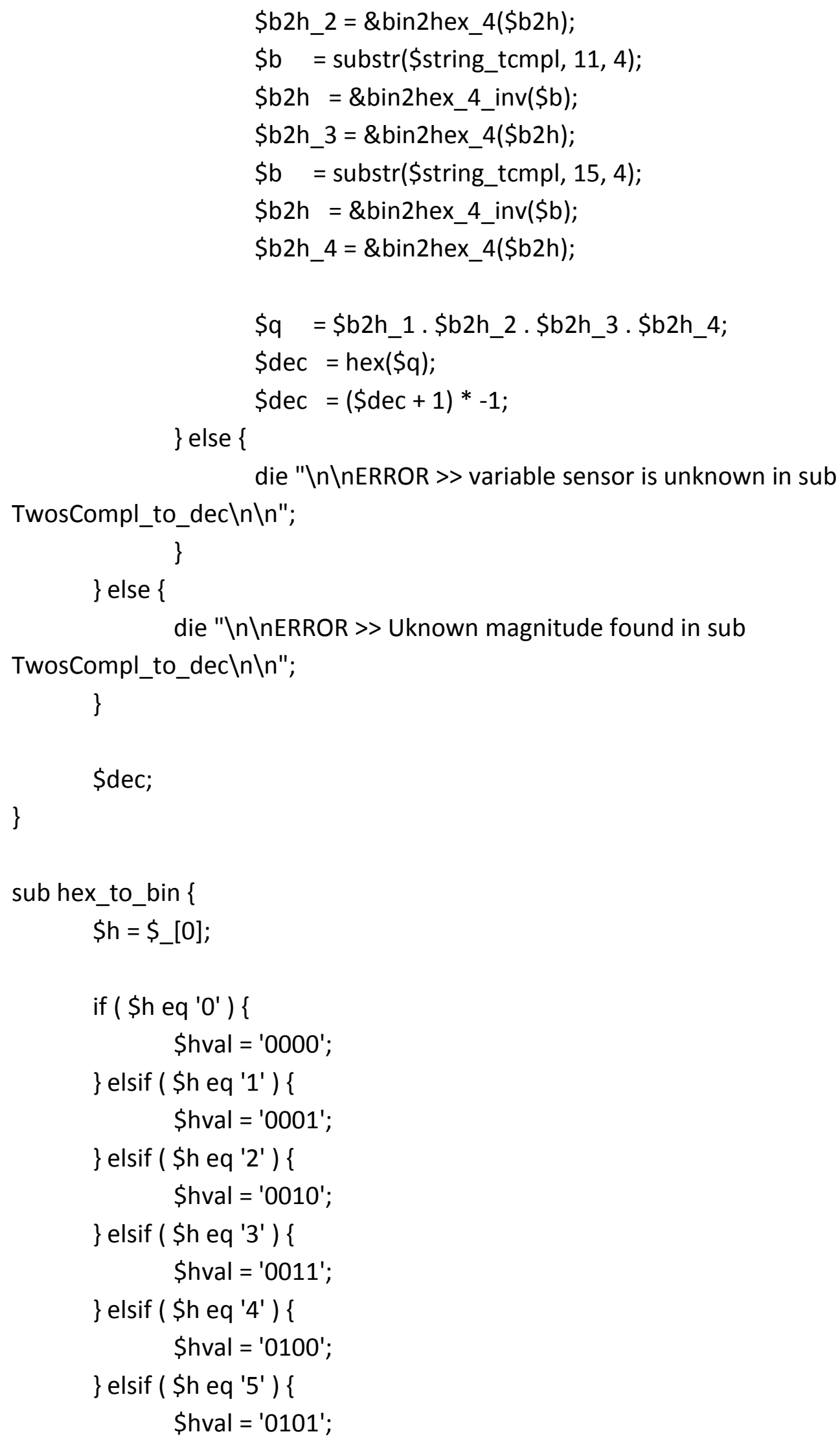




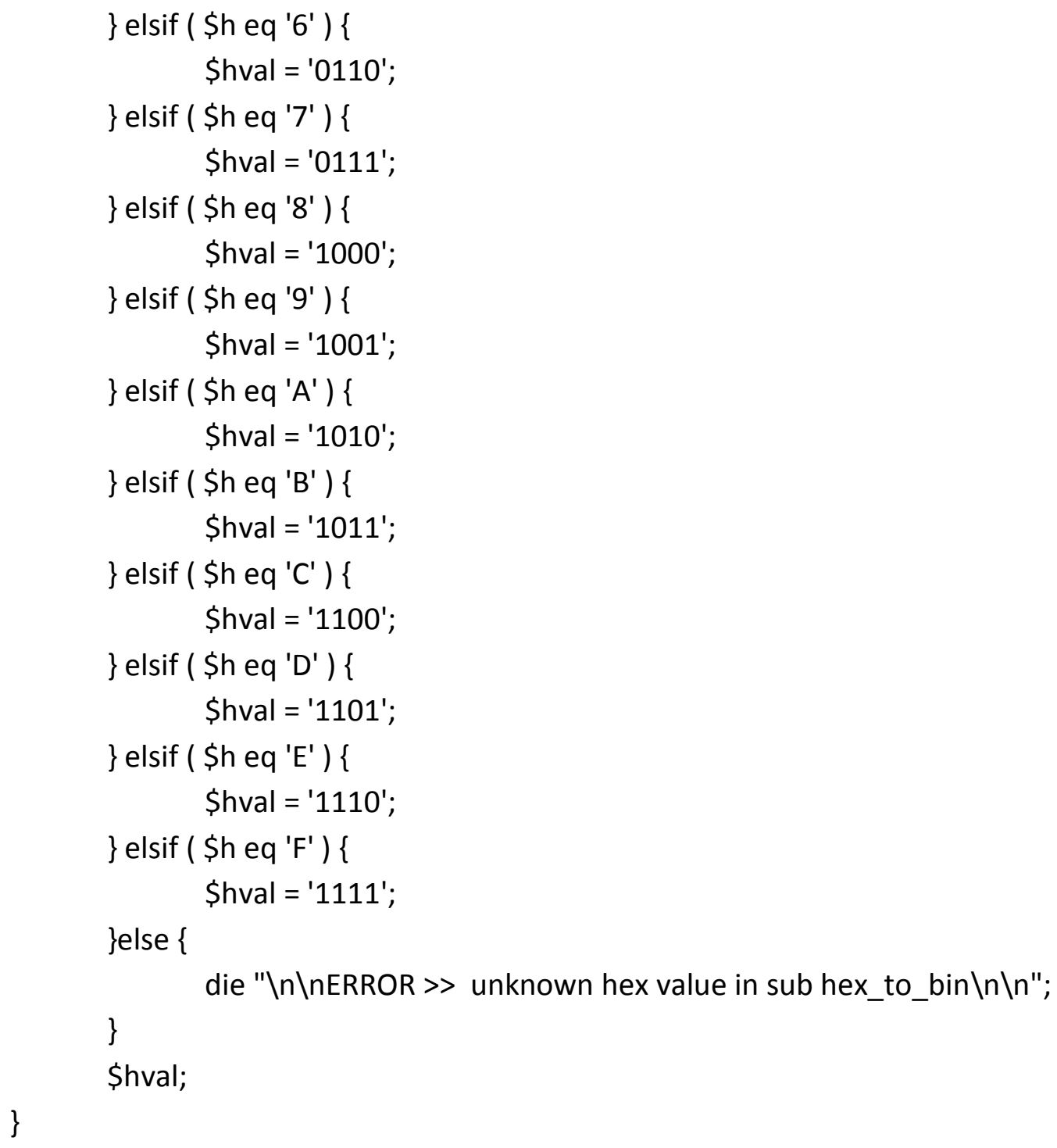




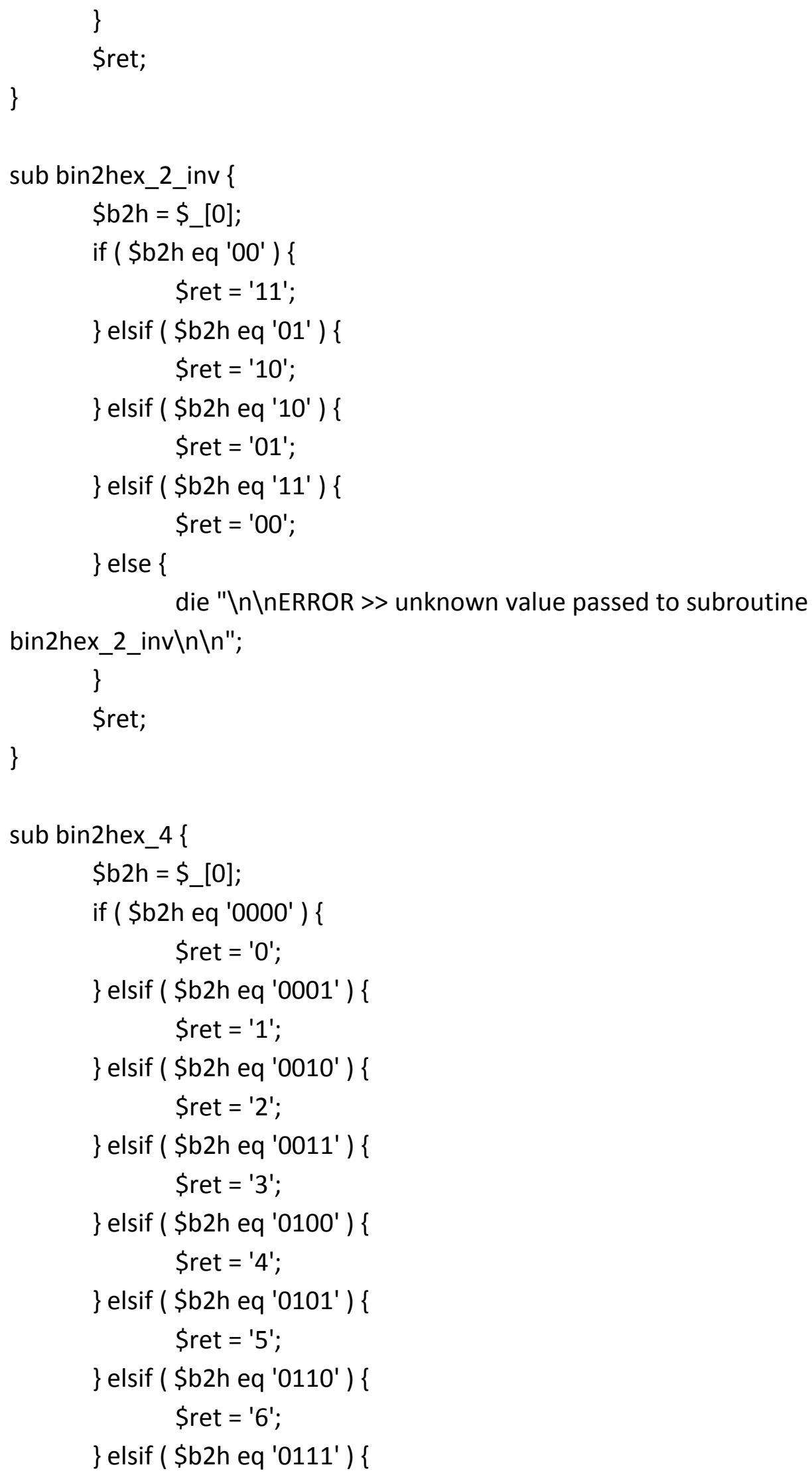




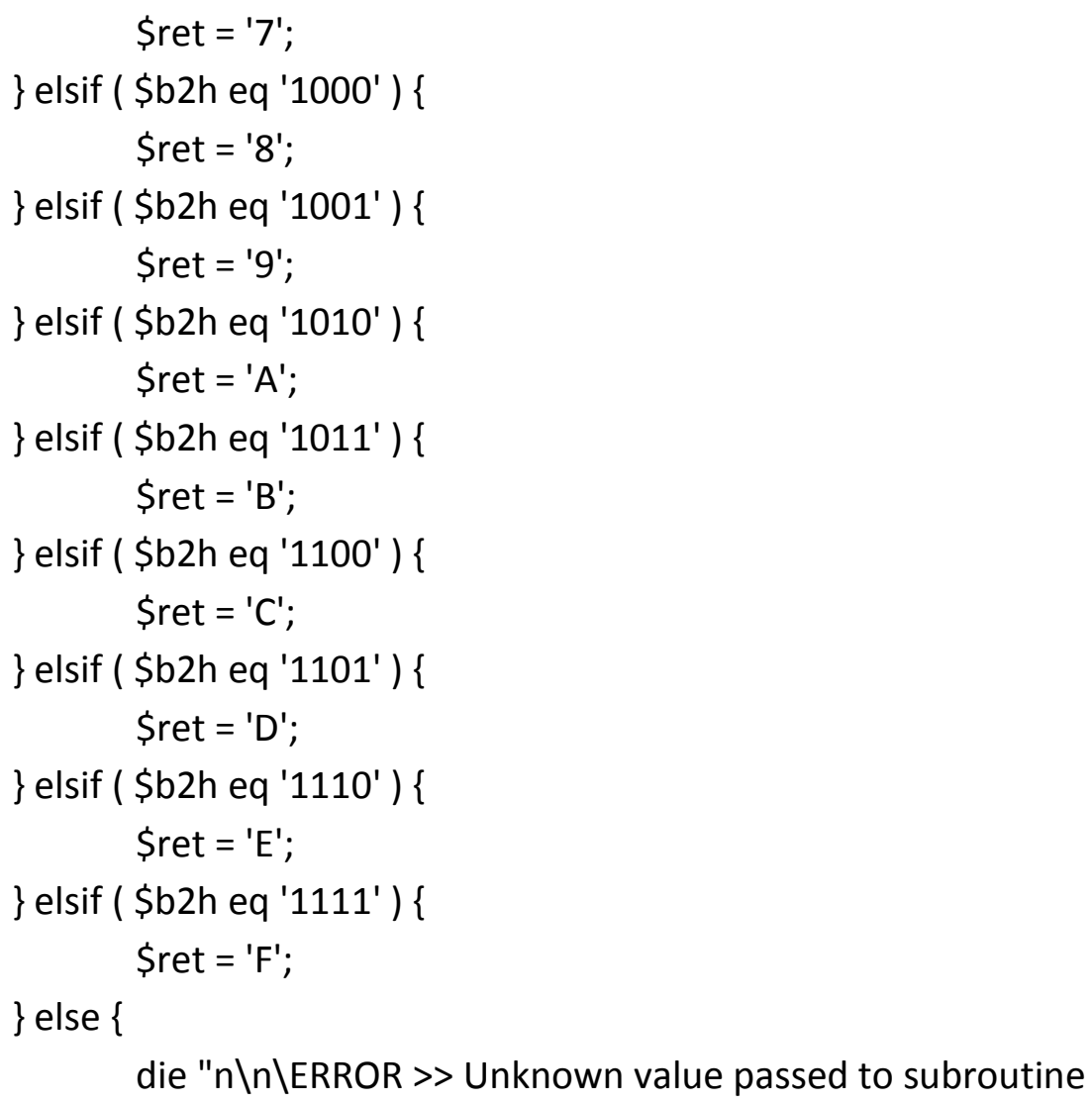




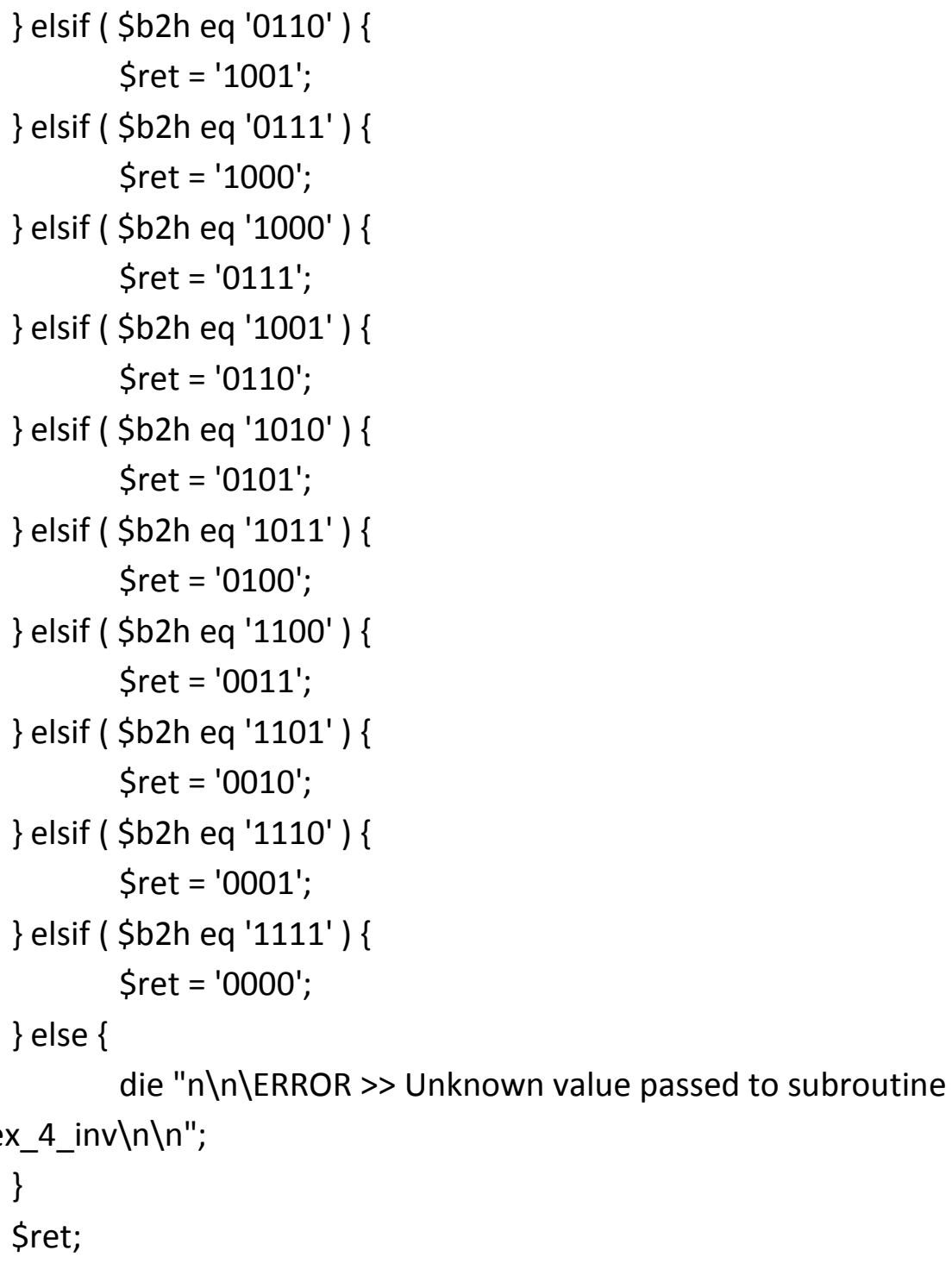




\section{References}

[1] M. Higgins and R. Whyte, "Controlled depressor towed sensor platform - the U.S. Navy's Mk28 search platform," in OCEANS '88. 'A Partnership of Marine Interests'. Proceedings, 1988, pp. 1646-1646.

[2] D. C. Echert, J. H. Morison, G. B. White, and E. W. Geller, "The Autonomous Ocean Profiler: a current-driven oceanographic sensor platform," Oceanic Engineering, IEEE Journal of, vol. 14, pp. 195-202, 1989.

[3] J. H. Morison, J. L. Backes, and C. W. May, "The Polar Ocean Profiler: A New Generation Of Oceanographic And Meteorological Sensor Platform," in OCEANS '91. 'Ocean Technologies and Opportunities in the Pacific for the 90's'. Proceedings., 1991, pp. 627-632.

[4] V. Pallayil, M. A. Chitre, and P. D. Deshpande, "A Digital Thin Line Towed Array for Small Autonomous Underwater Platforms," in OCEANS 2007, 2007, pp. 1-9.

[5] J. F. Vesecky, K. Laws, S. I. Petersen, C. Bazeghi, and D. Wiberg, "Prototype autonomous mini-buoy for use in a wireless networked, ocean surface sensor array," in Geoscience and Remote Sensing Symposium, 2007. IGARSS 2007. IEEE International, 2007, pp. 4987-4990.

[6] C. A. Grimes, M. K. Jain, R. S. Singh, Q. Cai, A. Mason, K. Takahata, and Y. Gianchandani, "Magnetoelastic microsensors for environmental monitoring," in Micro Electro Mechanical Systems, 2001. MEMS 2001. The 14th IEEE International Conference on, 2001, pp. 278-281.

[7] T. Ahonen, R. Virrankoski, and M. Elmusrati, "Greenhouse Monitoring with Wireless Sensor Network," in Mechtronic and Embedded Systems and Applications, 2008. MESA 2008. IEEE/ASME International Conference on, 2008, pp. 403-408.

[8] R. Beckwith, D. Teibel, and P. Bowen, "Unwired wine: sensor networks in vineyards," in Sensors, 2004. Proceedings of IEEE, 2004, pp. 561-564 vol.2.

[9] Y. Guo, P. Corke, G. Poulton, T. Wark, G. Bishop-Hurley, and D. Swain, "Animal Behaviour Understanding using Wireless Sensor Networks," in Local Computer Networks, Proceedings 2006 31st IEEE Conference on, 2006, pp. 607614.

[10] J. Hayes, S. Beirne, L. King-Tong, and D. Diamond, "Evaluation of a low cost wireless chemical sensor network for environmental monitoring," in Sensors, 2008 IEEE, 2008, pp. 530-533.

[11] J. Lifton, M. Feldmeier, Y. Ono, C. Lewis, and J. A. Paradiso, "A Platform for Ubiquitous Sensor Deployment in Occupational and Domestic Environments," in Information Processing in Sensor Networks, 2007. IPSN 2007. 6th International Symposium on, 2007, pp. 119-127.

[12] F. Tsow, E. Forzani, A. Rai, W. Rui, R. Tsui, S. Mastroianni, C. Knobbe, A. J. Gandolfi, and N. J. Tao, "A Wearable and Wireless Sensor System for Real-Time Monitoring of Toxic Environmental Volatile Organic Compounds," Sensors Journal, IEEE, vol. 9, pp. 1734-1740, 2009. 
[13] B. Alandry, P. Nouet, F. Mailly, and L. Latorre, "A MEMS-based multi-sensor platform for consumer applications," in Research in Microelectronics and Electronics, 2009. PRIME 2009. Ph.D., 2009, pp. 320-323.

[14] M. De Marinis, L. Fanucci, A. Giambastiani, A. Reneiri, A. Rocchi, C. Rosadini, C. Sicilia, and D. Sicilia, "Sensor platform design for automotive applications," in Digital System Design, 2003. Proceedings. Euromicro Symposium on, 2003, pp. 346-353.

[15] B. Carkhuff and R. Cain, "Corrosion sensors for concrete bridges," Instrumentation \& Measurement Magazine, IEEE, vol. 6, pp. 19-24, 2003.

[16] A. M. Madni, "Keynote speech:Smart configurable wireless sensors and actuators for industrial monitoring and control," in Sensors, 2009 IEEE, 2009, pp. 16581659.

[17] A. M. Madni, "Smart configurable wireless sensors and actuators for industrial monitoring and control," in Communications, Control and Signal Processing, 2008. ISCCSP 2008. 3rd International Symposium on, 2008, pp. 447-448.

[18] H. Ramamurthy, B. S. Prabhu, R. Gadh, and A. M. Madni, "Wireless Industrial Monitoring and Control Using a Smart Sensor Platform," Sensors Journal, IEEE, vol. 7, pp. 611-618, 2007.

[19] H. Ramamurthy, B. S. Prabhu, R. Gadh, and A. M. Madni, "Smart sensor platform for industrial monitoring and control," in Sensors, 2005 IEEE, 2005, p. 4 pp.

[20] P. Blythe, J. Neasham, B. Sharif, P. Watson, M. C. Bell, S. Edwards, V. Suresh, J. Wagner, and H. Bryan, "An environmental sensor system for pervasively monitoring road networks," in Road Transport Information and Control - RTIC 2008 and ITS United Kingdom Members' Conference, IET, 2008, pp. 1-10.

[21] E. Siwapornsathain, A. Lal, and J. Binard, "A telemetry and sensor platform for ambulatory urodynamics," in Microtechnologies in Medicine \& Biology 2nd Annual International IEEE-EMB Special Topic Conference on, 2002, pp. 283287.

[22] L. K. Au, W. H. Wu, M. A. Batalin, D. H. McLntire, and W. J. Kaiser, "MicroLEAP: Energy-aware Wireless Sensor Platform for Biomedical Sensing Applications," in Biomedical Circuits and Systems Conference, 2007. BIOCAS 2007. IEEE, 2007, pp. 158-162.

[23] Y. Hung-Chieh and T. Shu-Ming, "A Wireless Based Sensor for Patient Monitoring System with Remote Diagnostic," in Networking and Services, 2007. ICNS. Third International Conference on, 2007, pp. 92-92.

[24] A. Alshehab, N. Kobayash, R. Kikuchi, J. Ruiz, S. Shimamoto, and H. Ishibashi, "A study on intra-body communication for personal healthcare monitoring system," in e-health Networking, Applications and Services, 2008. HealthCom 2008. 10th International Conference on, 2008, pp. 219-220.

[25] P. van de Ven, A. Bourke, C. Tavares, R. Feld, J. Nelson, A. Rocha, and G. O. Laighin, "Integration of a suite of sensors in a wireless health sensor platform," in Sensors, 2009 IEEE, 2009, pp. 1678-1683.

[26] Y. Chuo, M. Marzencki, B. Hung, C. Jaggernauth, K. Tavakolian, P. Lin, and B. Kaminska, "Mechanically Flexible Wireless Multisensor Platform for Human 
Physical Activity and Vitals Monitoring," Biomedical Circuits and Systems, IEEE Transactions on, vol. 4, pp. 281-294, 2010.

[27] R. Szewczyk, E. Osterweil, J. Polastre, M. Hamilton, A. Mainwaring, and D. Estrin, "Habitat monitoring with sensor networks," Commun. ACM, vol. 47, pp. 34-40, 2004.

[28] X. Chen, M. Q. H. Meng, and H. Ren, "Design of Sensor Node Platform for Wireless Biomedical Sensor Networks," in Engineering in Medicine and Biology Society, 2005. IEEE-EMBS 2005. 27th Annual International Conference of the, 2005, pp. 4662-4665.

[29] G. Tolle, J. Polastre, R. Szewczyk, D. Culler, N. Turner, K. Tu, S. Burgess, T. Dawson, P. Buonadonna, D. Gay, and W. Hong, "A macroscope in the redwoods," presented at the Proceedings of the 3rd international conference on Embedded networked sensor systems, San Diego, California, USA, 2005.

[30] D. Hyun, H. S. Yang, H.-S. Park, and H.-J. Kim, "Dead-reckoning sensor system and tracking algorithm for 3-D pipeline mapping," Mechatronics, vol. 20, pp. 213-223, 2010.

[31] E. A. Johannessen, W. Lei, C. Wyse, D. R. S. Cumming, and J. M. Cooper, "Biocompatibility of a Lab-on-a-Pill Sensor in Artificial Gastrointestinal Environments," Biomedical Engineering, IEEE Transactions on, vol. 53, pp. 2333-2340, 2006.

[32] M. Ouwerkerk, F. Pasveer, and N. Engin, "SAND: a modular application development platform for miniature wireless sensors," in Wearable and Implantable Body Sensor Networks, 2006. BSN 2006. International Workshop on, 2006, pp. 5 pp.-170.

[33] W. Zheyao, W. Lianwei, N. T. Nguyen, W. A. H. Wien, H. Schellevis, P. M. Sarro, and J. N. Burghartz, "Silicon micromachining of high aspect ratio, highdensity through-wafer electrical interconnects for 3-D multichip packaging," Advanced Packaging, IEEE Transactions on, vol. 29, pp. 615-622, 2006.

[34] R. V. Martinez-Catala and J. Barrett, "A Modular Wireless Sensor Platform With Fully Integrated Battery," Components and Packaging Technologies, IEEE Transactions on, vol. 32, pp. 617-626, 2009.

[35] C. Jingkuang, C. Xiaoyang, I. M. Shen, L. Jian-Hung, L. Pai-Chi, and W. Mengli, "A Monolithic Three-Dimensional Ultrasonic Transducer Array for Medical Imaging," Microelectromechanical Systems, Journal of, vol. 16, pp. 1015-1024, 2007.

[36] A. Haahr, M. Kirkevold, E. O. C. Hall, and K. Ostergaard, "From miracle to reconciliation: A hermeneutic phenomenological study exploring the experience of living with Parkinson's disease following Deep Brain Stimulation," International Journal of Nursing Studies, vol. 47, pp. 1228-1236, 2010.

[37] B. H. Kopell, A. R. Rezai, J. W. Chang, and J. L. Vitek, "Anatomy and physiology of the basal ganglia: Implications for deep brain stimulation for Parkinson's disease," Movement Disorders, vol. 21, pp. S238-S246, 2006.

[38] S. Naskar, S. K. Sood, V. Goyal, and M. Dhara, "Mechanism(s) of deep brain stimulation and insights into cognitive outcomes in Parkinson's disease," Brain Research Reviews, vol. 65, pp. 1-13, 2010. 
[39] M. Tagliati, "Lack of Motor Symptoms Progression in Parkinson's Disease Patients With Long-Term Bilateral Subthalamic Deep Brain Stimulation," International Journal of Neuroscience, vol. 120, pp. 717-723, 2010.

[40] R. Coffey, "Deep Brain Stimulation for Chronic Pain: Results of Two Multicenter Trials and a Structured Review," Pain Medicine, vol. 2, pp. 183-192, 2001.

[41] T. Lovick, "Pain relief from deep brain stimulation at midbrain sites - A contribution from vagal processes?," Experimental Neurology, vol. 225, pp. 240242, 2010.

[42] S. L. F. Owen, A. L. Green, D. Nandi, R. G. Bittar, S. Wang, and T. Z. Aziz, "Deep Brain Stimulation for Neuropathic Pain," Neuromodulation, vol. 9, pp. 100-106, 2006.

[43] T. Wyckhuys, "Suppression of hippocampal epileptic seizures in the kainate rat by Poisson distributed stimulation T. Wyckhuys et al. Poisson Distributed Stimulation," Epilepsia (Series 4), vol. 51, pp. 2297-2304, 2010.

[44] C. Carlson, "Intractable Epilepsy: Relapsing, Remitting, or Progressive?," Epilepsy Currents, vol. 10, pp. 148-150, 2010.

[45] I. Najm, "Deep Brain Stimulation for Epilepsy," Neuromodulation, vol. 12, pp. 270-280, 2009.

[46] D. E. Hardesty and H. A. Sackeim, "Deep Brain Stimulation in Movement and Psychiatric Disorders," Biological Psychiatry, vol. 61, pp. 831-835, 2007.

[47] C. Loo, "Mental health legislation and psychiatric treatments in NSW: electroconvulsive therapy and deep brain stimulation," Australasian Psychiatry, vol. 18, pp. 417-425, 2010.

[48] N. Lipsman, R. S. McIntyre, P. Giacobbe, C. Torres, S. H. Kennedy, and A. M. Lozano, "Neurosurgical treatment of bipolar depression: defining treatment resistance and identifying surgical targets," Bipolar Disorders, vol. 12, pp. 691$701,2010$.

[49] K. E. Hoy and P. B. Fitzgerald, "Spreading activation: the origins of brain stimulation in psychiatry," Acta Neuropsychiatrica, vol. 22, pp. 302-304, 2010.

[50] C. R. Butson and C. C. McIntyre, "Role of electrode design on the volume of tissue activated during deep brain stimulation," Journal of Neural Engineering, vol. 3, pp. 1-8, 2006.

[51] C. C. McIntyre, S. Mori, D. L. Sherman, N. V. Thakor, and J. L. Vitek, "Electric field and stimulating influence generated by deep brain stimulation of the subthalamic nucleus," Clinical Neurophysiology, vol. 115, pp. 589-595, 2004.

[52] X. F. Wei and W. M. Grill, "Current density distributions, field distributions and impedance analysis of segmented deep brain stimulation electrodes," Journal of Neural Engineering, vol. 2, pp. 139-147, 2005.

[53] J. B. J. Schulz, "Deep brain stimulation," Cell and Tissue Research, vol. 318, pp. 275-288, 2004.

[54] J. Holsheimer, "Theoretical Investigation Into Longitudinal Cathodal Field Steering in Spinal Cord Stimulation," Neuromodulation, vol. 10, pp. 120-132, 2007.

[55] L. Litvak, "Current focusing and steering: Modeling, physiology, and psychophysics," Hearing Research, vol. 242, pp. 141-153, 2008. 
[56] C. McIntyre, "Current steering to control the volume of tissue activated during deep brain stimulation," Brain Stimulation, vol. 1, pp. 7-15, 2008.

[57] N. Laotaveerungrueng, L. Chia-Hua, G. McCallum, S. Rajgopal, C. P. Steiner, A. R. Rezai, and M. Mehregany, "3-D microfabricated electrodes for targeted deep brain stimulation," in Engineering in Medicine and Biology Society, 2009. EMBC 2009. Annual International Conference of the IEEE, 2009, pp. 6493-6496.

[58] Z. Cao and D. M. Aslam, "Smart All-Diamond Packaging for WIMS," WIMS ERC Annual Report 2009, p. 83, 2009.

[59] B. Gyselinckx, C. Van Hoof, J. Ryckaert, R. F. Yazicioglu, P. Fiorini, and V. Leonov, "Human++: autonomous wireless sensors for body area networks," in Custom Integrated Circuits Conference, 2005. Proceedings of the IEEE 2005, 2005, pp. 13-19.

[60] J. M. Rabaey, J. Ammer, T. Karalar, L. Suetfei, B. Otis, M. Sheets, and T. Tuan, "PicoRadios for wireless sensor networks: the next challenge in ultra-low power design," in Solid-State Circuits Conference, 2002. Digest of Technical Papers. ISSCC. 2002 IEEE International, 2002, pp. 200-201 vol.1.

[61] L. Nazhandali, B. Zhai, A. Olson, A. Reeves, M. Minuth, R. Helfand, P. Sanjay, T. Austin, and D. Blaauw, "Energy optimization of subthreshold-voltage sensor network processors," in Computer Architecture, 2005. ISCA '05. Proceedings. 32nd International Symposium on, 2005, pp. 197-207.

[62] T. Salter, G. Metze, and N. Goldsman, "Parasitic aware optimization of an RF power scavenging circuit with applications to Smartdust sensor networks," in Radio and Wireless Symposium, 2009. RWS '09. IEEE, 2009, pp. 332-335.

[63] A. B. Ucok, J. M. Giachino, and K. Najafi, "Modular assembly/packaging of multi-substrate microsystems (WIMS Cube) using thermo-magnetically actuated cables," in Micro Electro Mechanical Systems, 2005. MEMS 2005. 18th IEEE International Conference on, 2005, pp. 536-539.

[64] J. F. Hetke, J. L. Lund, K. Najafi, K. D. Wise, and D. J. Anderson, "Silicon ribbon cables for chronically implantable microelectrode arrays," Biomedical Engineering, IEEE Transactions on, vol. 41, pp. 314-321, 1994.

[65] Z. Honghai, M. Maozhou, S. Xiayun, and L. Sheng, "Design of a Flexible Stethoscope Sensor Skin Based on MEMS Technology," in Electronic Packaging Technology, 2006. ICEPT '06. 7th International Conference on, 2006, pp. 1-4.

[66] R. B. Katragadda and X. Yong, "A novel intelligent textile technology based on silicon flexible skins," in Micro Electro Mechanical Systems, 2007. MEMS. IEEE 20th International Conference on, 2007, pp. 301-304.

[67] L. F. Velasquez-Garcia, A. I. Akinwande, and M. Martinez-Sanchez, "Precision Hand Assembly of MEMS Subsystems Using DRIE-Patterned Deflection Spring Structures: An Example of an Out-of-Plane Substrate Assembly," Microelectromechanical Systems, Journal of, vol. 16, pp. 598-612, 2007.

[68] S. M. Hu, "Critical stress in silicon brittle fracture, and effect of ion implantation and other surface treatments," Journal of Applied Physics, vol. 53, pp. 3576-3580, 1982.

[69] S. Johansson, F. Ericson, and J.-A. r. Schweitz, "Influence of surface coatings on elasticity, residual stresses, and fracture properties of silicon microelements," Journal of Applied Physics, vol. 65, pp. 122-128, 1989. 
[70] W. C. Young and R. G. Budynas, Roark's Formulas for Stress and Strain, 7th ed.: McGraw-Hill, 2002.

[71] J. H. Davies, MSP430 Microcontroller Basics. Oxford: Elsevier, 2008.

[72] D. H. Titterton and J. L. Weston, "Strapdown Inertial Navigation Technology (2nd Edition)," ed: Institution of Engineering and Technology, 2004.

[73] BMA020 Digital, triaxial acceleration sensor - Data Sheet v.1.2. Available: www.bosch-sensortec.com

[74] ITG-3200 Product Specification Revision 1.4. Available: www.invensense.com

[75] A. Babar, L. Ukkonen, and L. Sydanheimo, "Dual UHF RFID band miniaturized multipurpose planar antenna for compact wireless systems," in Antenna Technology (iWAT), 2010 International Workshop on, 2010, pp. 1-4.

[76] F. Ohnimus, R. Erxleben, C. Tschoban, I. Ndip, M. Niedermayer, H. Scholtz, T. Bonim, S. Guttowski, and K. D. Lang, "Design and characterization of a low profile miniaturized UHF PIFA for compact wireless sensor nodes," in Antennas and Propagation Conference (LAPC), 2010 Loughborough, 2010, pp. 257-260.

[77] C. Kruesi and M. M. Tentzeris, "'Magic-Cube" antenna configurations for ultra compact RFID and wireless sensor nodes," in Antennas and Propagation Society International Symposium, 2008. AP-S 2008. IEEE, 2008, pp. 1-4.

[78] A. Rydberg, P. van Engen, C. Shi, R. van Doremalen, M. Sanduleanu, K. Hjort, W. De Raedt, T. Fritzsch, and P. Hallbjorner, "Body surface backed flexible antennas and 3D Si-level integrated wireless sensor nodes for $17 \mathrm{GHz}$ wireless body area networks," in Antennas and Propagation for Body-Centric Wireless Communications, 2009 2nd IET Seminar on, 2009, pp. 1-4.

[79] N. Haridas, A. T. Erdogan, T. Arslan, and M. Begbie, "Adaptive Micro-Antenna on Silicon Substrate," in Adaptive Hardware and Systems, 2006. AHS 2006. First NASA/ESA Conference on, 2006, pp. 43-50.

[80] C. Calvez, C. Person, J. Coupez, Galle, x, F. e, F. Gianesello, D. Gloria, D. Belot, and H. Ezzeddine, "Packaged hybrid Si-IPD(TM) Antenna for $60 \mathrm{GHz}$ applications," in Microwave Conference (EuMC), 2010 European, 2010, pp. 683686.

[81] S. Brebels, K. Mohammadpour-Aghdam, W. De Raedt, and G. Vandenbosch, "3D system-in-package integration of $60 \mathrm{GHz}$ aperture-coupled micromachined microstrip antennas," in Microwave Symposium Digest (MTT), 2010 IEEE MTT-S International, 2010, pp. 1028-1031.

[82] S. B. Yeap, Z. N. Chen, L. Rui, H. D. S. W., and L. T. Guan, "135-GHz co-planar patch array on $\mathrm{BCB} /$ silicon with polymer-filled cavity," in Antenna Technology (iWAT), 2011 International Workshop on, 2011, pp. 344-347.

[83] C. Person, "Antennas on Silicon for millimeterwave applications - Status and trends," in Bipolar/BiCMOS Circuits and Technology Meeting (BCTM), 2010 IEEE, 2010, pp. 180-183.

[84] S. Eunyoung, S. Dongha, M. Chuying, H. Ruonan, S. Sankaran, C. Changhua, W. Knap, and K. O. Kenneth, "Progress and Challenges Towards Terahertz CMOS Integrated Circuits," Solid-State Circuits, IEEE Journal of, vol. 45, pp. 1554$1564,2010$. 